\title{
Du bien-être des nations
}

\section{LE RÔLE DU CAPITAL HUMAIN ET SOCIAL}

ENSEIGNEMENT ET COMPÉTENCES

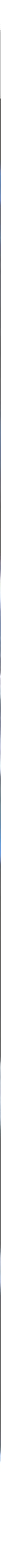


Centre pour la recherche et l'innovation dans l'enseignement

\section{Du bien-être des nations \\ LE RÔLE DU CAPITAL HUMAIN ET SOCIAL}




\section{ORGANISATION DE COOPÉRATION ET DE DÉVELOPPEMENT ÉCONOMIQUES}

En vertu de l'article $1^{\mathrm{er}}$ de la Convention signée le 14 décemb re 1960, à Paris, et entrée en vigueur le 30 septembre 1961, l'Organisation de Coopération et de Développement Économiques (OCDE) a pour objectif de promouvoir des politiques visant :

- à réaliser la plus forte expansion de l'économie et de l'emploi et une progression du niveau de vie dans les pays Membres, tout en maintenant la stabilité financière, et à contribuer ainsi au développement de l'économie mondiale ;

- à contribuer à une saine expansion économique dans les pays Membres, ainsi que les pays non membres, en voie de développement économique ;

- à contribuer à l'expansion du commerce mondial sur une base multilatérale et non discriminatoire conformément aux obligations internationales.

Les pays Membres originaires de I'OCDE sont : I'Allemagne, l'Autriche, la Belgique, le Canada, le Danemark, I'Espagne, les États-Unis, la France, la Grèce, I'Irlande, I'Islande, I'Italie, le Luxembourg, la Norvège, les Pays-Bas, le Portugal, le Royaume-Uni, la Suède, la Suisse et la Turquie. Les pays suivants sont ultérieurement devenus Membres par adhésion aux dates indiquées ci-après : le Jap on (28 avril 1964), la Finlande (28 janvier 1969), I'Australie (7 juin 1971), la Nouvelle-Zélande (29 mai 1973), le Mexique (18 mai 1994), la République tchèque (21 décembre 1995), la Hongrie (7 mai 1996), la Pologne (22 novembre 1996), la Corée (12 décembre 1996) et la République slovaque (14 décembre 2000). La Commission des Communautés européennes participe aux travaux de I'OCDE (article 13 de la Convention de I'OCDE).

Le Centre pour la Recherche et l'Innovation dans l'E nseignement a étécréé par le Conseil de l'O rganisation de Coopération et de D éveloppement É conomiques en juin 1968 et tous les pays M embres de l'O CDE y participent.

Les principaux objectifs du Centre sont les suivants :

- de poursuivre les travaux de recherche et d'analy se sur les innovations et les indicateurs clés afin de mieux appréhender les problèmes d'enseignement et d'apprentissage existants ou qui se font jour, ainsi que leurs liens avec les autres domaines d'action ;

- d'explorer des stratégies d'enseignement et d'apprentissage cohérentes et prometteuses qui tiennent compte de l'évolution du contexte économique, social et culturel aux niveaux national et international ; et

- de faciliter la coopération pratique entre les pays M embres et, si nécessaire avec les pays non membres, afin qu'ils recherchent des solutions à des problèmes éducatifs communs et échangent leurs points de vue sur ces problèmes.

Le Centre exerce son activité au sein de l'O rganisation de Coopération et de D éveloppement É conomiques conformément aux décisions du Conseil de l'O rganisation, sous l'autorité du Secrétaire général et le contrôle direct d'un Comité directeur composé d'experts nationaux dans le domaine de compétence du Centre, chaque pays participant étant représenté par un expert.

A Iso available in English under the title:
THE WELL-BEING OF NATIONS
The Role of H uman and Social Capital

(C) OCDE 2001

Les permissions de reproduction partielle à usage non commercial ou destinée à une formation doivent être adressées au Centre français d'exploitation du droit de copie (CFC), 20, rue des Grands-Augustins, 75006 Paris, France, tél. (33-1) 440747 70, fax (33-1) 463467 19, pour tous les pays à l'exception des États-Unis. Aux États-Unis, l'autorisation doit être obtenue du Copyright Clearance Center, Service Client, (508)750-8400, 222 Rosewood Drive, Danvers, M A 01923 USA, or CCC Online: www. copyright.com. Toute autre demande d'autorisation de reproduction ou de traduction totale ou partielle de cette publication doit être adressée aux Éditions de l'OCDE, 2, rue André-Pascal, 75775 Paris Cedex 16, France. 


\section{AVANT-PROPOS}

Ce rapport est une publication du Centre pour la recherche et l'innovation dans l'enseignement de I'OCDE. Ses deux auteurs, Tom Healy et Sylvain Côté, ont bénéficié du précieux concours de John F. Helliwell (Université de Colombie Britannique, Canada), de Simon Field (CERI/OCDE) et de beaucoup de leurs collègues au Secrétariat de I'OCDE.

Cette étude a largement profité des conseils formulés par un grand nombre d'experts universitaires et de représentants nationaux lors des réunions des comités de l'OCDE, qui ont orienté l'évolution des travaux. Deux manifestations extérieures ont également revêtu une importance particulière : premièrement, un symposium international organisé et co-parrainé par Développement des Ressources Humaines Canada et I'OCDE sur La contribution du capital humain et social à la croissance économique et au bien-être, en mars 2000 à Québec, Canada ; deuxièmement, une réunion d'un groupe d'experts sur le capital humain et social, organisée à I'OCDE, en juillet 2000, pour examiner le premier projet du présent rapport.

Ce volume est publié sous la responsabilité du Secrétaire général de I'OCDE. 


\section{Remerciements}

Parmi les experts internationaux qui ont participé à l'élaboration de ce rapport, nous tenons tout particulièrement à remercier : Gunnar Eliasson (Institut royal de technologie de Stockholm), Dominique Foray (Université Dauphine, Paris), David Halpern (Université de Cambridge, Royaume-Uni), Tom Kellaghan (Centre de recherche en éducation, Irlande), Bengt-Aake Lundwall (Université d'Aalborg, Danemark), Peter MacDonagh (Conseiller près le Premier ministre d'Irlande), Lars Osberg (Université de Dalhousie, Canada), Robert D. Putnam (Université d'Harvard, États-Unis), Jo Ritzen (Vice-Président, Banque mondiale), Tom Schuller (Birkbeck College, Royaume-Uni), Simon Szreter (Université de Cambridge, Royaume-Uni), Jonathan Temple (Oxford College, Royaume-Uni), Doug Willms (Université du Nouveau-Brunswick, Canada), Michael Woolcock (Banque mondiale), Jean-Pierre Worms (Centre de sociologie des organisations, Paris).

Nous adressons nos plus vifs remerciements à Développement des Ressources Humaines Canada (DRHC), en particulier à Jean-Pierre Voyer et Richard Roy, pour l'aide apportée à divers stades du projet, le financement du détachement de Sylvain Côté près le Secrétariat de I'OCDE et le co-parrainage du symposium organisé à Québec, en mars 2000. 


\section{TABLE DES MATIÈRES}

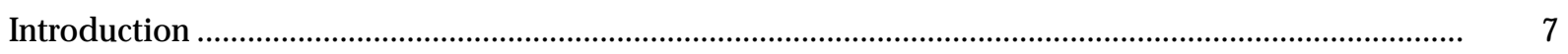

Chapitre 1. Nouvelles préoccupations sociales et économiques.......................................................

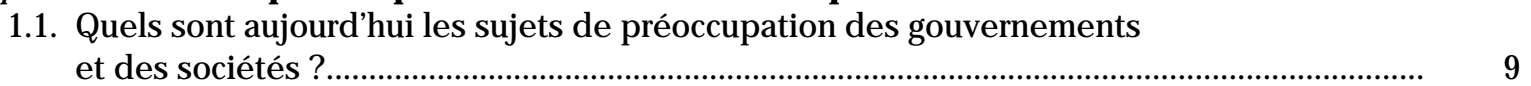

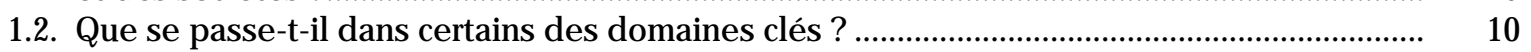

1.3. L'interaction entre le bien-être et le capital humain et social ................................................. 12

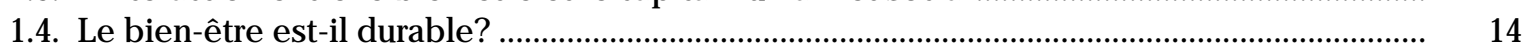

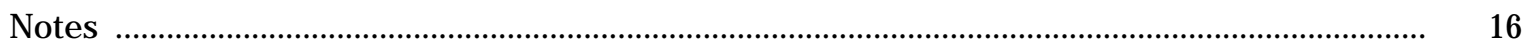

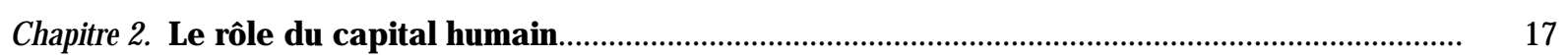

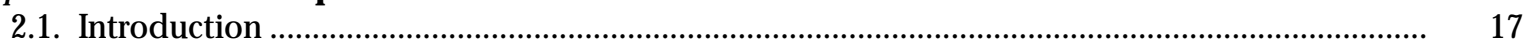

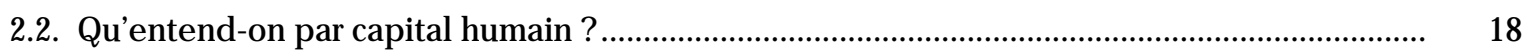

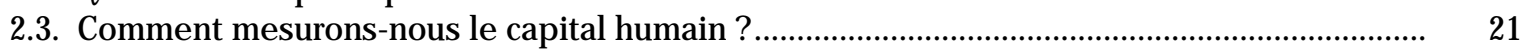

2.4. Comment le capital humain se forme-t-il ? ........................................................................... 21

2.5. Comment le capital humain est-il réparti ? ........................................................................... 26

2.6. L'évolution de la demande de capital humain ........................................................................ 29

2.7. Quel est l'impact du capital humain sur le bien-être économique ? ...................................... 30

2.8. Quelle est l'incidence du capital humain sur tous les aspects du bien-être ? ....................... 36

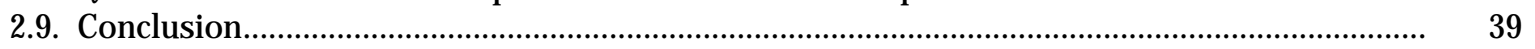

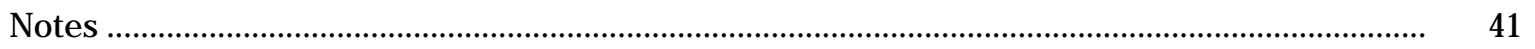

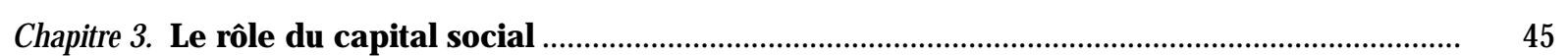

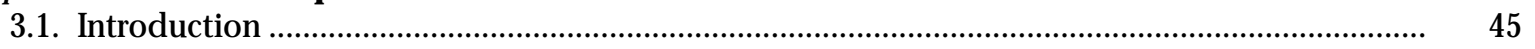

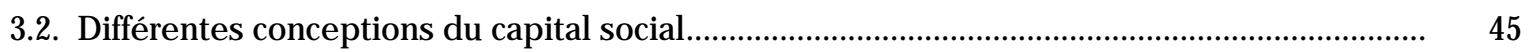

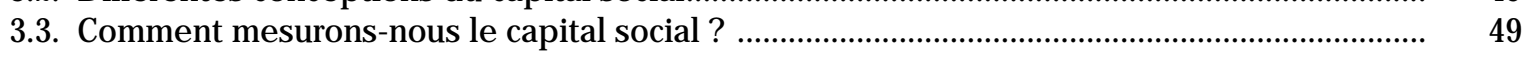

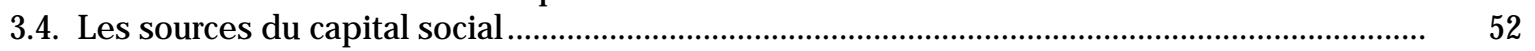

3.5. La confiance et la participation à la vie civique évoluent-elles au fil du temps ? ................. 55

3.6. Quelle est l'origine de l'évolution des niveaux de confiance

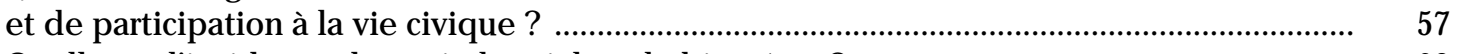

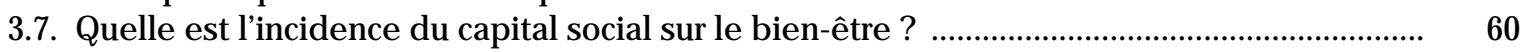

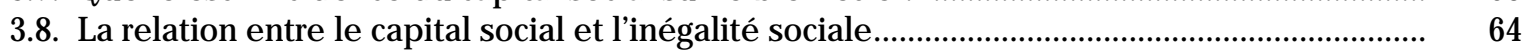

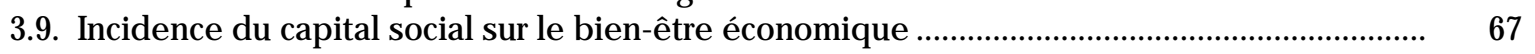

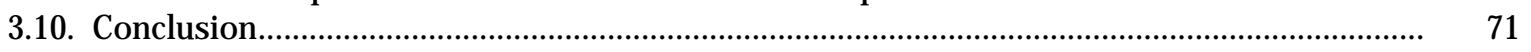

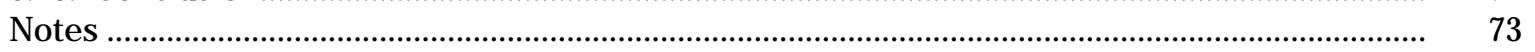

Chapitre 4. Conséquences pour l'action des gouvernements et autres recherches à effectuer ......... 77

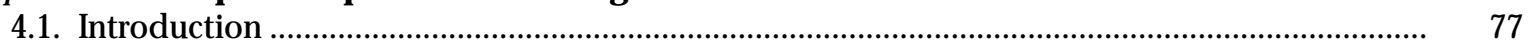

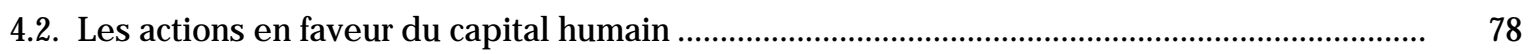

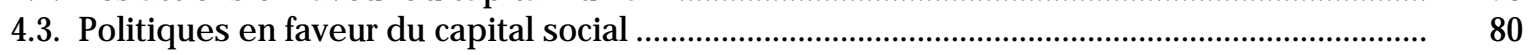

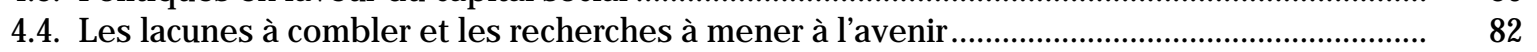

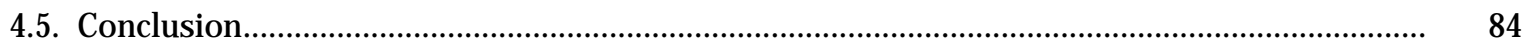

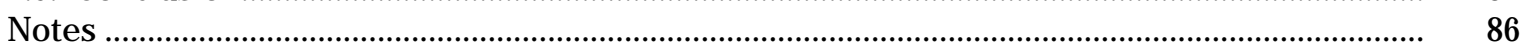


Annexe A. Quelques indicateurs du bien-être.

Annexe B. Évolution de l'environnement social et économique - Quelques tendances ....................... 95

Annexe C. Déterminants du niveau scolaire : les résultats des recherches.......................................... 105

Annexe D. L'incidence du capital humain sur la croissance économique : quelques grandes études.

Annexe E. La confiance et l'engagement civique sont-ils en baisse dans les pays de I'OCDE ? ........... 115

Bibliographie

Liste des graphiques

1.1 Rapport entre le bien-être humain, le bien-être économique et le PIB

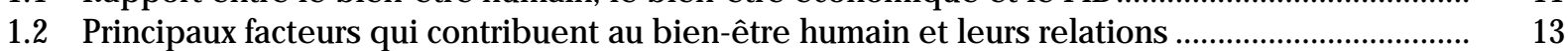

2.1 Répartition comparative des niveaux de littératie................................................................... 22

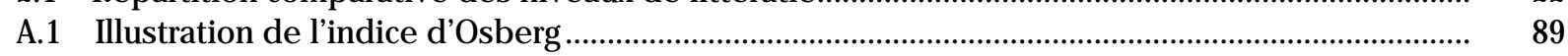

A.2 Comparaisons internationales des tendances du bien-être économique .................................. 91

B.1 Produit intérieur brut réel par habitant à prix constant, moyenne de l'OCDE, 1966-99 ............... 95

B.2 Tendances de l'inégalité des revenus pour l'ensemble de la population dans certains pays de I'OCDE, milieu des années 80 et 90 .

B.3 Pourcentage d'enfants vivant dans une pauvreté relative dans certains pays de I'OCDE, années 90

B.4 Taux de chômage des 25-54 ans et des 15-24 ans dans certains pays de I'OCDE, 1975-99.......... 98

B.5 Taux de dépendance dû à l'âge dans les pays de l'OCDE, 1950-2050 ........................................... 98

B.6 Pourcentage de la population des pays de l'OCDE de 25 à 64 ans

ayant un niveau égal ou supérieur à la fin des études secondaires, 1950-98

B.7 Parents isolés dans certains pays de l'OCDE, comparaison entre les années 80 et $90 \ldots \ldots \ldots \ldots . . . . . . \quad 99$

B.8 Écart de rémunération entre les hommes et les femmes de 25 à 64 ans

(travaillant à plein temps), années 80 et 90 .

B.9 Différence de taux d'activité entre les hommes et les femmes de 15 à 64 ans dans certains pays de l'OCDE, 1960-99....

B.10 Flux d'entrées d'étrangers dans quelques pays de I'OCDE, 1999

101

B.11 Nombre de décès par toxicomanie enregistrés dans l'Union européenne par million d'habitants, 1986-97

101

B.12 Pourcentage de la population victime de délinquance, années 80 et 90

102

B.13 Espérance moyenne de vie à la naissance dans certains pays de I'OCDE, 1960-98 ....................... 102

B.14 Tendances récentes de la satisfaction personnelle dans 12 pays de l'Union européenne, 1973, 1983 et 1997.

B.15 Proportion de personnes donnant différentes réponses sur le bonheur, États-Unis, 1972-98

B.16 Taux de suicide dans certains pays de l'OCDE, 1950-97....

104

B.17 Émissions de gaz à effet de serre dans les pays de l'OCDE selon différents scénarios, 1990-2010.

E.1 Baisse du nombre de réunions de clubs aux États-Unis, 1975-1999 .......................................... 115

E.2 Personnel employé à la justice et à la sécurité aux États-Unis, 1900-1997 .................................. 116

Liste des tableaux

3.1 Mesure de la confiance (World Values Study), 1995-96.

D.1 L'impact de la formation dans les analyses de régression transversales : quelques études phares. 


\section{INTRODUCTION}

Le rôle du capital humain dans le développement économique et social est un thème ancien même si I'on continue de polémiquer au sujet de son importance exacte. Ces dernières années, on s'est aussi de plus en plus intéressé au rôle du capital social - il s'agit dans ce cas d'étudier l'idée selon laquelle les relations sociales ainsi que les caractéristiques personnelles contribuent de façon primordiale à l'activité économique et au bien-être.

Le présent rapport a une triple finalité : i) décrire les toutes dernières données recueillies au sujet de l'investissement dans le capital humain et de son impact sur la croissance et le bien-être ; ii) définir et préciser la notion plus novatrice du capital social ; et iii) déterminer les fonctions du capital humain et social dans la réalisation du développement économique et social durable. Ce rap port contribue aux projets de I'OCDE sur la croissance économique et le développement durable. II fait également suite au rapport de l'OCDE intitulé L'investissement dans le capital humain - Une comparaison internationale et publié en 1998.

Le chapitre 1 décrit les principaux problèmes, tendances, concepts et relations analysés dans le rap port et les grandes questions à traiter. Le chapitre 2 examine la définition et la mesure du capital humain, comprenant tous les types de compétences et de caractéristiques individuelles qui influent sur le bien-être. On y analyse les sources de capital humain se trouvant dans les familles, les écoles, les collectivités et les lieux de travail, en insistant sur la façon dont les possibilités de formation et les conséquences de ce processus se répartissent entre différents groupes. Ce chapitre évalue aussi les données empiriques disponibles sur l'impact du capital humain sur l'économie et la société. Le chapitre 3 traite de la définition et de la mesure du capital social et examine ses origines et ses effets sur toute une gamme de résultats possibles. Il analyse le potentiel de complémentarité entre le capital social et le capital humain, d'un point de vue conceptuel et d'un point de vue empirique. Le chapitre 4 examine certaines questions fondamentales sur les conséquences qu'ont pour les politiques gouvernementales les analyses du capital humain et du capital social précédentes et définit d'autres recherches à mener et données à recueillir au niveau international. L'une des considérations essentielles est que de multiples acteurs entrent en jeu lorsqu'il s'agit de favoriser la réalisation d'investissements de qualité dans les personnes, les organisations ou les collectivités, et d'en tirer le meilleur profit possible. 



\section{Chapitre 1}

\section{NOUVELLES PRÉOCCUPATIONS SOCIALES ET ÉCONOMIQUES}

« II ne faut pas perdre de vue les distinctions entre le volume et la qualité de la croissance, entre ses coûts et son rendement et entre le court terme et le long terme... Lorsqu'on vise « plus » de croissance, il faudrait préciser plus de croissance de quoi et pour quoi ». Simon Kuznets dans The N ew Republic (1962).

Dans le présent rapport, le capital humain et le capital social sont considérés non pas comme des fins en soi, mais comme des ressources qui peuvent être utilisées pour favoriser le développement économique et social. L'objet dans ce chapitre est de situer le débat dans le contexte de ces objectifs sociaux plus vastes.

\subsection{Quels sont aujourd'hui les sujets de préoccupation des gouver- nements et des sociétés?}

Les pouvoirs publics et la société recherchent la croissance économique, mais ils se préoccupent aussi de plus en plus de son incidence sur l'environnement naturel et social. Par ailleurs, ils s'inquiètent de l'inégalité, des nouvelles formes possibles d'exclusion et de pauvreté à mesure que l'utilisation des technologies se développent et, d'une façon plus générale, de la qualité de vie et de la santé des enfants, des personnes âgées, ainsi que des individus et des groupes souffrant d'un handicap économique ou social. Apporter une solution à ces préoccupations est d'autant plus délicat qu'évoluent les modes de travail, de vie familiale et d'engagement dans la collectivité.

Ces préoccupations naissantes résultent de l'évolution fondamentale des valeurs et des modes d'engagement social, qui se produit dans de nombreux pays de I'OCDE et pourrait nécessiter une réévaluation des objectifs de l'action gouvernementale. Bien souvent, cette évolution dénote une plus grande diversité et une plus grande place faite à l'individualité, à l'autonomie et à la responsabilité personnelle, au bien-être subjectif et à la qualité de vie. La World Values Study donne à penser qu'au-delà d'un certain seuil de revenu par habitant, la progression du bien-être subjectif ralentit à mesure que le revenu augmente (Inglehart, 1997).

De nombreux observateurs ont examiné le point d'articulation entre le progrès économique et les dysfonctionnements sociaux - par exemple, les conséquences pour les travailleurs de l'évolution rapide des technologies, de l'obsolescence des qualifications, de l'insécurité dans l'emploi et de l'allongement du temps de travail. II n'est pas établi dans le présent rapport que l'augmentation de la prospérité économique ait nécessaire-
L'insertion sociale et la qualité de la vie comptent parmi les grandes préoccupations des pouvoirs publics et de la société...

... et les valeurs et les attitudes subissent des changements radicaux.

Le progrès économique et social ne sont pas nécessairement contradictoires... 
... mais nous devons nous inquiéter de l'évolution à long terme du bien-être, ainsi que de la progression à court terme de la production économique.

Une croissance économique rapide réduit la pauvreté absolue...

... mais le bien-être est une notion plus générale que le bien-être économique... ment amenuisé les réserves de capital social, mais l'étude conclut qu'il pourrait exister un lien entre certains aspects du progrès économique et une augmentation du stress ou un relâchement des liens sociaux. Toutefois, les nouvelles conditions de production, d'organisation et de travail créent de nouvelles possibilités qui peuvent, si elles sont exploitées, conduire à une nette amélioration du bien-être.

Dans le domaine social, les objectifs sont plus généraux que celui qui vise à accroître dans l'immédiat la production économique ; en effet : i) l'évolution du bien-être global, et non pas uniquement économique, revêt de l'importance, et ii) les conséquences à long terme des tend ances économiques, environnementales et sociales doivent être prises en considération dans toute analyse des possibilités d'action actuelles. Le facteur temps est crucial lorsqu'on considère les schémas actuels d'investissement et de production et les effets futurs à en attendre en aval, du fait que de nombreux choix d'orientation publics et privés se caractérisent par des incertitudes et de longues périodes de gestation. Pour faire progresser le bien-être dans la durée, il importe de comprendre les effets que pourrait avoir l'évolution de l'environnement humain et social, mais aussi physique et naturel.

\subsection{Que se passe-t-il dans certains des domaines clés ?}

Depuis plusieurs décennies, les pays de I'OCDE connaissent de fortes augmentations de leur production économique, accompagnées d'améliorations des niveaux de vie, des conditions de travail, de la santé et de l'éducation. Même si la progression de la prospérité matérielle n'a pas profité à tout le monde dans la même mesure, les niveaux de pauvreté absolue et de dénuement ont baissé dans les pays de I'OCDE depuis les années 50. La croissance économique n'est pas le seul objectif des pouvoirs publics, mais elle procure effectivement des ressources qui permettent de s'attaquer à l'exclusion sociale, à la pauvreté et à la mauvaise santé. Après des augmentations sans précédent de la production économique, on s'inquiète à présent de la «qualité » de la croissance économique et des moyens à mettre en œuvre pour obtenir de nouvelles améliorations du bien-être1.

Dans le graphique 1.1, on a définit trois niveaux de bien-être2. Le bien-être englobe certes le bien-être économique, mais également la jouissance des libertés civiles, d'un environnement propre, d'un bon état de santé mental et physique et l'absence relative de criminalité. Dans le même ordre d'idée, Sen (1987) insiste sur les « aptitudes sociales » des individus ou encore les possibilités qui s'offrent à eux de choisir et d'atteindre les objectifs qui leur conviennent le mieux. Dans cette démarche, la croissance de la production économique, au lieu d'être un objectif en soi, élargit la palette des choix qui s'offrent à l'être humain (par exemple, travail, loisirs ou activités politiques et culturelles). Les résultats atteints (les « performances » selon la terminologie de Sen) comptent moins que les « possibilités de faire » permettant aux individus de mener la vie qui leur convient et qu'ils ont choisie. II est donc essentiel d'utiliser les capacités humaines pour élargir la notion et l'évaluation du développement humain et social. Le bien-être humain est plus que la somme des différents niveaux de bien-être car il est lié aux préférences individuelles et socié- 


\section{Graphique 1.1. Rapport entre le bien-être humain, le bien-être économique et le PIB}

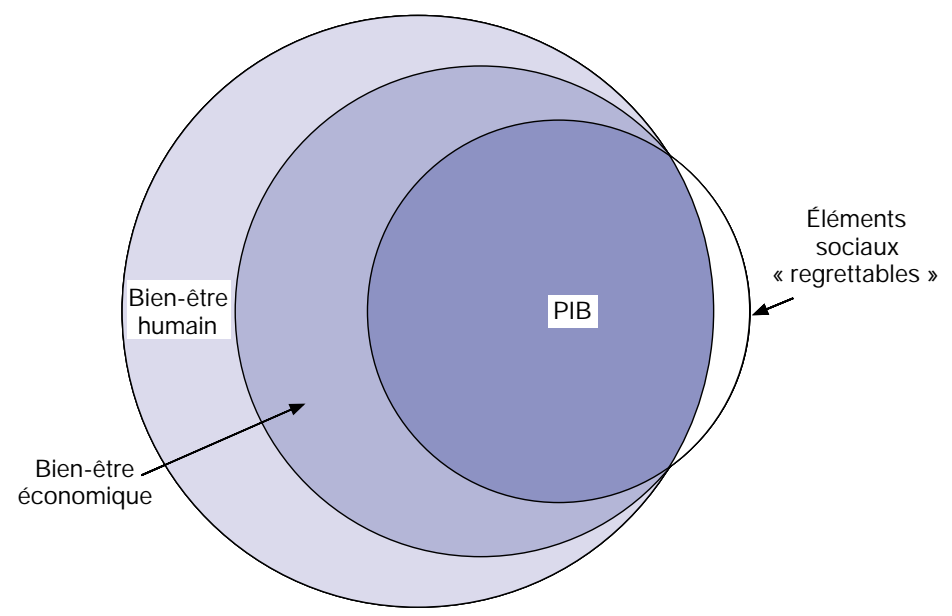

Source : OCDE.

tales à l'égard de l'égalité des chances, des libertés civiles, de la répartition des ressources et des possibilités de toujours apprendre plus.

Le bien-être économique - fruit de la production économique - est une composante importante du bien-être. Toutefois, le produit intérieur brut (PIB) 3 p résente des limites non négl igeables lorsqu'il s'agit de mesurer le produit économique. Le PIB rend compte de la production actuelle des biens de consommation et d'équipement et des services figurant dans la comptabilité nationale, mais exclut les activités non marchandes des ménages 4 (telles que l'éducation des enfants) et ainsi que les activités de conservation des ressources naturelles qui contribuent au bien-être futur grâce à des additions nettes au patrimoine de la société5. Les mesures globales de la production et du revenu, telles que le PIB, ne reflètent pas non plus les préférences de la société concernant les objectifs d'équité.

Le PIB englobe des biens et des services qui ne contribuent pas directement au bien-être. Les éléments dits « regrettables » découlant de la pollution, de la criminalité et de l'éclatement des familles en sont des exemples. Dans le graphique 1.1, les «éléments regrettables » font partie du $\mathrm{PIB}$, mais sont à l'extérieur du bien-être (y compris le bien-être économique). Les «éléments regrettables » sont rep résentés par des dépenses qui ne contribuent pas directement au bien-être, mais qui sont néanmoins jugées nécessaires par exemple pour la sécurité nationale (voir à l'annexe A).

Ce que nous entendons par bien-être ou épanouissement individuel est fonction de valeurs qui varient selon les individus et les groupes sociaux. L'évaluation des différents aspects du bien-être soulève également des difficultés techniques. Des aspects subjectifs tels que les niveaux déclarés de satisfaction et de bien-être personnel sont difficiles à mesurer ou à rattacher à des causes précises 6 . Lorsqu'on définit les besoins sociaux, il faut décider de la manière d'évaluer les besoins de différents groupes de la société civile. Par exemple, une inégalité des revenus peut être souhaitable pour inciter la population à travailler et traduire éven-
... et le bien-être économique ne se résume pas à la mesure du PIB notamment.

Le PIB comprend des activités qui ne contribuent pas directement au bien-être.

Nous ne pouvons espérer un consensus clair sur la signification du bien-être... 
... ce qui n'a pas cependant empêché de tenter d'élaborer des mesures synthétiques.

Ces mesures donnent à penser que le bien-être progresse plus lentement que le PIB. tuellement aussi les préférences individuelles à l'égard d'un style de vie donné, d'un lieu de résidence, d'un métier, d'un équilibre entre loisirs et travail ou entre une activité bénévole et un emploi rémunéré. Pour mettre en lumière les grandes tendances sociales, il est possible de choisir des indicateurs différents et d'attribuer implicitement à chacun des pondérations ou des degrés d'importance (voir annexe A).

En dépit de ces difficultés, plusieurs méthodes ont été essayées pour calculer une mesure synthétique du bien-être. L'une d'elles (Osberg, 2001) porte uniquement sur le bien-être économique et réunit quatre principaux types d'indicateurs : i) les flux actuels de consommation par habitant ; ii) les variations des stocks de capital (naturel et humain); iii) les variations de la répartition des revenus; et iv) l'évolution des risques économiques.

Le bien-être dans certains pays de l'OCDE semble progresser plus lentement que le PIB par habitant (voir l'annexe A). Le graphique A.2 de l'annexe présente les résultats de ces calculs pour cinq pays. Les mesures d'Osberg montrent que jusqu'aux années 80, l'évolution du PIB par habitant suit de très près celle du bien-être économique dans de nombreux pays de l'OCDE, mais qu'elles ont eu tendance à diverger depuis lors. De même, d'autres mesures établies à partir d'une gamme plus large d'indicateurs sociaux (par exemple, l'indice du bien-être économique durable et l'indice de la santé sociale de Fordham) révèlent la même évolution depuis la période située entre le début et le milieu des années 80 . Cette divergence s'explique principalement par la dégradation de l'environnement, l'augmentation de la pauvreté relative et les inégalités de revenu dans certains pays de I'OCDE.

Les tendances économiques et sociales sont liées, mais en partie seulement. Dans le graphique A.1, on a ind iqué l'évolution tend ancielle dans toute une série de domaines sociaux, pour tous les pays de I'OCDE ou certains d'entre eux, depuis le milieu des années 70 ou une époque plus tardive. II s'agit d'indicateurs de variation des revenus, de la pauvreté, de la participation au marché du travail ainsi que de l'évolution des schémas de composition de la famille, et de la santé, de la démographie et de l'environnement.

\subsection{L'interaction entre le bien-être et le capital humain et social}

Le rôle des différents facteurs qui influent sur le bien-être et les liens complexes existant entre tous ces éléments sont illustrés dans le graphique 1.2 .

Dans le graphique 1.2, du côté des «facteurs contribuant au bien-être » figurent le capital naturel et physique ainsi que les «capacités humaines et sociales ». Le capital humain représente les connaissances, les aptitudes et la santé des personnes (voir la définition dans le chapitre 2 ) et le capital social désigne les normes et les réseaux qui facilitent la coopération au sein des groupes ou entre eux (voir la définition dans le chapitre 3). Les dispositions politiques, institutionnelles et juridiques interagissent avec le capital humain et social et, ce faisant, influent sur le bien-être. En outre, le capital humain et social ainsi que les dispositions politiques, institutionnelles et juridiques ont leurs propres liens traditionnels directs avec le 


\section{Graphique 1.2. Principaux facteurs qui contribuent au bien-être humain et leurs relations}

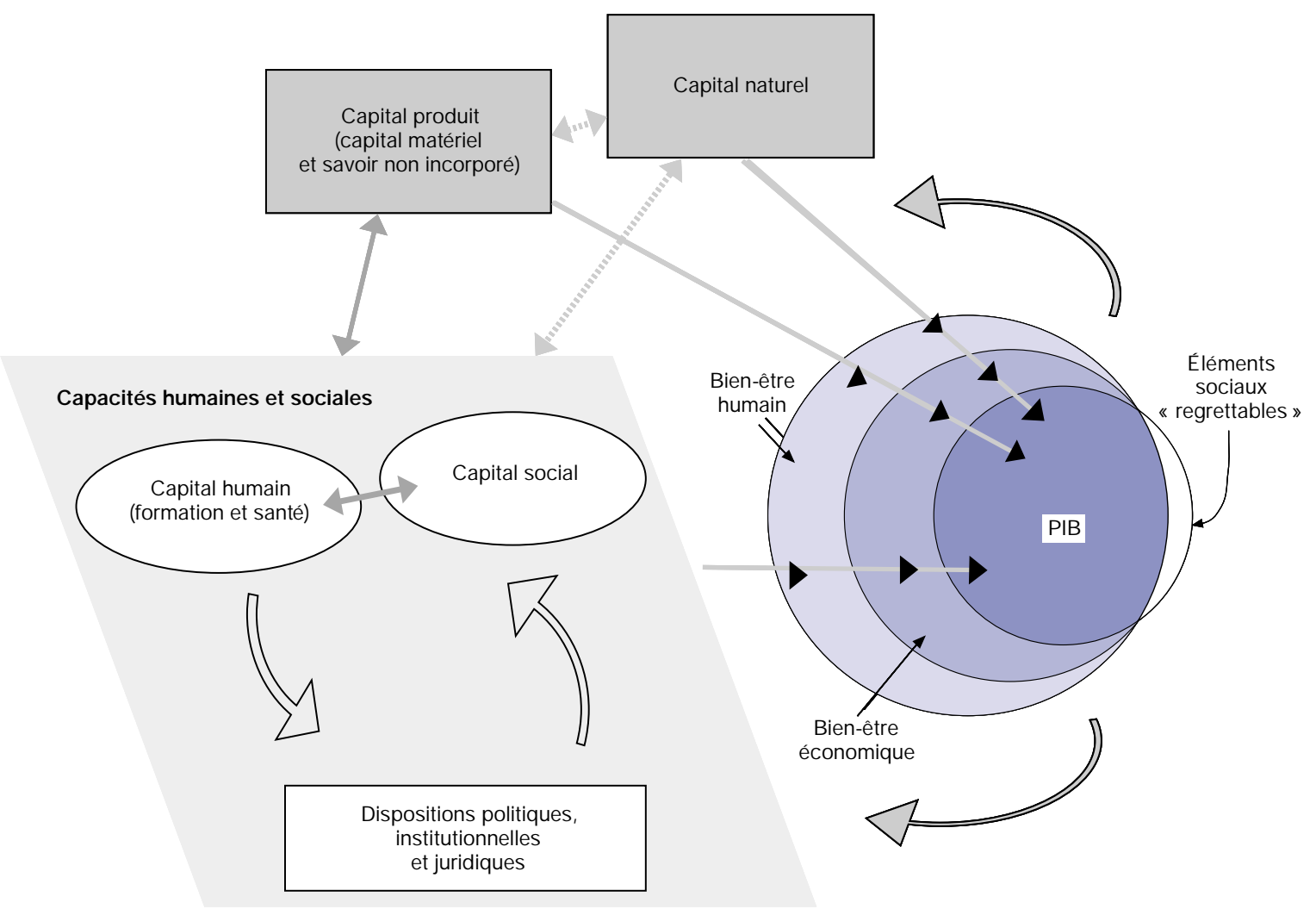

Source : OCDE.

capital naturel et le capital produit, mais ces liens ne sont pas signalés dans le graphique pour éviter une complexité excessive.

Il peut y avoir une forte complémentarité entre le capital humain, le capital social et les dispositions politiques, institutionnelles et juridiques7. Dans les travaux qu'il a consacrés au capital social dans les années 80 , Coleman (1988) souligne le rôle que jouent les communautés fortes et des liens solides entre les parents, les éducateurs et les élèves pour faciliter l'acquisition de connaissances. Par ailleurs, les activités d'enseignement et d'apprentissage peuvent favoriser les habitudes, les qualifications et les valeurs propices à la coopération et à la participation sociale. Des institutions de qualité, une main-d'œuvre hautement qualifiée et l'existence de normes et de réseaux facilitant la coopération servent souvent de base à des investissements plus élevés dans l'équipement et peuvent aussi renforcer les stratégies de régénération du milieu naturel. La santé est aussi un facteur important du bien-être et de la performance économique tout en étant liée à l'âge, au mode de vie, à la situation sociale, au niveau de formation et à l'étendue des liens sociaux et de la solidarité entre les personnes. De fait, certains économistes considèrent que la santé fait partie du capital humain8.

Les activités commerciales et la vie civique reposent sur toute une série d'institutions civiques, politiques et juridiques, officielles ou non. Les institutions fixent les « règles du jeu ». Rodrik (2000) décrit cinq types d'institutions qui :
... et les dispositions politiques, institutionnelles et juridiques sont des compléments importants du capital humain et social. 
Il faut établir une nette distinction entre le capital humain, le capital social et les dispositions politiques, institutionnelles et juridiques.
- protègent la propriété privée et le respect des contrats ;

- modèrent certaines activités économiques ;

- soutiennent la stabilité macro-économique ;

- offrent une assurance ou une protection sociale ; et

- gèrent les conflits sociaux.

Ces institutions, lorsqu'elles fonctionnent efficacement, peuvent permettre aux pays se trouvant à des niveaux différents de développement de gérer le changement et de parvenir à une croissance économique soutenue9.

La notion de cohésion sociale est très proche de celle de capital social. Selon la définition de Jenson (1998), la cohésion sociale s'entend " des valeurs partagées et du sentiment d'ap partenance à la collectivité » et se caractérise par cinq aspects importants : I'appartenance, l'intégration, la participation, la reconnaissance et la légitimité. Les sociétés présentant une plus grande cohésion parviennent à atteind re leurs objectifs globaux car elles réussissent mieux à protéger et à intégrer les personnes et les groupes en danger d'exclusion. Ritzen (2001) stipule que «l'objectif de cohésion sociale exige de concilier un système d'organisation fondé sur les forces du marché, la liberté de choisir et l'entreprise d'une part, et un attachement aux valeurs de solidarité et de soutien mutuel qui assure le libre accès aux avantages et à la protection pour tous les membres de la société d'autre part ». Cette acception de la cohésion sociale correspond à des situations d'harmonie ou décrit les retombées de situations de ce genre, qui résultent de divers facteurs, dont le capital humain et le capital social. La notion de cohésion sociale est donc plus générale que celle de capital social.

Le capital humain et le capital social sont étroitement liés à l'influence qu'exercent les institutions et les dispositions politiques et sociales sur la société. II faut cependant soigneusement distinguer plusieurs éléments car :

- le capital humain réside dans les individus ;

- le capital social réside dans les relations sociales ; et

- les dispositions politiques, institutionnelles et juridiques définissent les règlements et les institutions en vertu desquels le capital humain et capital social fonctionnent.

\subsection{Le bien-être est-il durable?}

II faut parfois beaucoup de temps pour que certains effets du capital Un bien-être durable exige des investissements suffisants dans le capital humain et dans le capital social... humain et du capital social sur le bien-être se fassent sentir ; il en est bien évidemment de même des avantages à long terme des investissements affectés par la société aux enfants. Un niveau insuffisant d'investissement peut compromettre les chances des générations futures. II est difficile 
d'envisager les « besoins sociaux » futurs et cette démarche met en relief la nécessité de coordonner les différents domaines d'intervention des pouvoirs publics. Les décideurs accordent une importance de plus en plus grande au développement durable, ce qui montre qu'ils se préoccupent davantage de l'articulation entre les politiques environnementales et les besoins futurs.

Il y a lieu de mieux intégrer les questions concernant l'environnement économique, social et naturel, car, quelle que soit la politique envisagée, il faut établir un lien entre les choix d'investissement actuels et les tendances et coûts et avantages à long terme et s'intéresser à l'interdépendance de différents processus. Dans le rapport intérimaire relatif au projet triennal de I'OCDE sur le développement durable (OCDE, 1999b, p. 2), il est stipulé que la «notion de [développement durable] a aujourd'hui acquis un sens plus large, qui implique que les objectifs d'accroissement de l'efficience économique et de la richesse matérielle doivent prendre en compte les préoccupations sociales et environnementales dans le cad re plus général de l'action gouvernementale ».

Toute dégradation de l'environnement social se produira sans doute progressivement et touchera certains groupes plus que d'autres. Cette dégradation peut prendre la forme d'une insécurité accrue, d'une augmentation des comportements anti-sociaux, dont la délinquance, d'un allongement des déplacements entre le travail et le domicile ou d'une baisse du bien-être personnel10. Une partie de cette dégradation, comme la réduction des niveaux d'engagement civique, peut même ne pas être visible dans un premier temps.

La cohésion sociale peut permettre à une société de mobiliser l'énergie d'une importante fraction de la population pour faire bouger les choses. Selon Rodrik (1998), la polarisation sociale peut empêcher l'économie de réagir à des chocs économiques néfastes. Un fossé grandissant entre les personnes très qualifiées et non qualifiées peut compromettre la cohésion sociale. Le capital humain et le capital social peuvent jouer un rôle important en facilitant l'utilisation efficace des compétences, le partage de l'information et la médiation des conflits d'intérêt. Comme le fait observer Dobell (2001, p. 37) : «A partir du moment où les pouvoirs s'exercent de nouveau par l'intermédiaire de la société civile et des institutions démocratiques, les décisions économiques qui ont des conséquences importantes en matière de répartition ne peuvent être prises uniquement sur la base de calculs économiques ni sur la base d'une analyse globale des coûts-avantages ou autres critères indirects. Pour que la société approuve l'action économique et la poursuite de la croissance économique, il faudra que l'action soit acceptable ou légitime aux yeux de la société civile qui est désormais dotée de pouvoirs. »
... dans la mesure où les changements de l'environnement social et de l'environnement naturel s'étalent sur de longues périodes.

\section{Partenariat social et consensus sont par conséquent nécessaires à un développement soutenu.}




\section{Notes}

1. Ces préoccupations ne sont pas nouvelles. Elles ont déjà été exp rimées dans les années 70 ap rès la croissance économique sans précédent de l'après-guerre. L'OCDE a lancé un Programme d'indicateurs sociaux dans les années 70, à la suite d'une déclaration ministérielle de 1970 soulignant que « la croissance n'est pas une fin en elle-même mais plutôt un moyen de créer des conditions de vie meilleures... il importe de prêter davantage d'attention à ses aspects qualitatifs et de définir les politiques à suivre à l'égard des grandes options économiques et sociales qu'implique l'allocation de ressources croissantes » (cité dans OCDE, 1976, p. 7).

2. Le bien-être économique est entièrement compris dans le terme de «bien-être ». Dans la suite du rapport, le terme «bien-être » désignera le bien-être humain à moins que le concept ne soit limité aux aspects économiques, auquel cas on utilisera l'expression «bien-être économique».

3. Les chercheurs qui ont contribué à l'élaboration des normes nationales de comptabilité au milieu du 20e siècle ont reconnu les limites des mesures existantes reposant sur le PIB.

4. La valeur du travail des femmes au foyer n'a pas été comptabilisée parce que ce travail ne fait pas partie d'un marché, erreur qui représente environ 8000 milliards de dollars (Picciotto, 1998).

5. Le PIB comprend certes l'investissement brut dans l'équipement ainsi que la consommation actuelle, mais il ne tient pas compte de la contribution qu'apporte la production actuelle aux changements d'autres types de patrimoine.

6. Toutefois, dans les chapitres 2 et 3 , nous donnerons les résultats récents d'analyses des niveaux de bien-être subjectif ou de satisfaction qui donnent à penser qu'on peut repérer de manière empirique des facteurs contribuant aux niveaux de bien-être subjectif.

7. Parfois, le capital humain, le capital social et les dispositions politiques, institutionnelles et juridiques sont substituables notamment lorsque les institutions et règlements officiels peuvent remplacer des réseaux sociaux informels.

8. Gary Becker, qui a été l'un des premiers à utiliser l'expression de « capital humain », assimilait l'instruction, la formation en cours d'emploi et la santé à des éléments du capital humain ayant des conséquences pour les gains et la productivité économique (Becker, 1993, pp. 54-55).

9. Toutefois, comme le souligne Rodrik, les mesures à prendre varient selon les pays et il n'existe pas de recettes magiques pouvant s'appliquer à toutes les situations et à tous les pays.

10. Une éventuelle détérioration de la santé est une question à part. Il peut suffire que de nombreuses personnes aient l'impression que leur bien-être a diminué pour que cela soit préoccupant. 


\section{Chapitre 2}

\section{LE RÔLE DU CAPITAL HUMAIN}

« La cohésion sociale est nécessaire pour que la croissance et la prospérité soient durables; là encore le rôle du capital humain est essentiel. Ces principes sont de plus en plus généralement admis... » (OCDE, 1998).

«Si l'enseignement a des effets sur la productivité de la main-d'œuvre, ce n'est pas sa vocation exclusive. ... On n'enseigne pas l'éducation civique, les lettres ou la musique uniquement pour améliorer la productivité au travail des apprenants, mais pour leur permettre de s'épanouir dans la vie et de mieux exercer leur qualité de citoyen. » (Weiss, 1995).

\subsection{Introduction}

Du fait de l'évolution des conditions économiques et sociales, les connaissances et les qualifications - le capital humain - jouent un rôle de plus en plus décisif dans la réussite économique des nations et des personnes. Les technologies de l'information et des communications, la mondialisation de l'activité économique et la tendance des individus à assumer davantage de responsabilités et à devenir plus autonome ont modifié la demande de formation. Les économistes et autres spécial istes admettent généralement que les compétences et le savoir jouent un rôle essentiel en stimulant la croissance économique.

Nombreux sont ceux qui considèrent que le rendement non économique de la formation, sous forme d'une amélioration du bien-être individuel et de la cohésion sociale, est aussi important que son effet sur les revenus du travail et sur la croissance économique. Ces objectifs individuels et collectifs de la formation ne sont pas nécessairement incompatibles avec l'amélioration visée des performances économiques, d'autant que la réalisation des objectifs économiques de l'éducation exige des individus qu'ils soient accomplis, souples et adaptables, et disposés à apprendre tout au long de leur vie.

Ce chapitre a pour objet de définir le capital humain, illustrations à l'appui, et d'étudier l'intérêt que présentent quelques questions de fond essentielles parmi lesquelles:

- les dispositions efficaces pour promouvoir des activités de formation donnant de bons résultats (section 2.4) ;

- I'impact de différents types de formation (niveau, domaine ou type de qualifications) sur la croissance du PIB (sections 2.7 et 2.8) ;

- la question de l'éventuel sous-investissement dans le capital humain du fait que parfois les personnes qui effectuent l'investissement ne

Les connaissances et les qualifications revêtent une importance économique croissante...

... mais les effets sociaux de la formation sont tout aussi importants que les effets économiques. 
sont pas celles qui profitent des avantages d'ordre social qui en découlent ;

- les effets de la formation et de l'enseignement sur le bien-être (sections 2.7 et 2.8).

\subsection{Qu'entend-on par capital humain ?}

Le capital humain s'entend des savoirs et savoir-faire que possède l'individu...

... et de ses caractéristiques individuelles.

La formation se déroule tout au long de la vie et dans toutes les facettes de la vie...
Dans le passé, les économistes distinguaient trois facteurs de production : la terre, le travail et le capital physique. A partir du début des années 60 , l'attention s'est de plus en plus portée sur la qualité de la main-d'œuvre, en particulier son niveau de formation. C'est ainsi qu'est apparue la notion de capital humain, qui désigne les qualifications et autres caractéristiques des personnes qui leur confèrent divers avantages d'ordre personnel, économique et social. Les qualifications et les compétences sont en grande partie acquises par l'instruction et l'expérience, mais peuvent également refléter des capacités innées. Certains aspects de la motivation et du comportement ainsi que des caractéristiques individuelles telles que la santé physique, psychologique et mentale sont aussi assimilés à du capital humain. La définition du capital humain utilisée dans ce rapport est la suivante :

Les connaissances, les qualifications, les compétences et caractéristiques individuelles qui facilitent la création de bien-être personnel, social et économique.

S'il est vrai que le «capital humain » a souvent été défini et évalué par rapport à des compétences cognitives acquises et à des savoirs précis, une notion plus générale du capital humain, englobant les caractéristiques individuelles, montre plus adéquatement comment diverses capacités non cognitives et autres caractéristiques individuelles contribuent au bienêtre et peuvent être influencées et modifiées par le milieu extérieur, notamment l'apprentissage. Le capital humain se développe dans des cadres culturels précis1.

L'acquisition de connaissances et de qualifications se déroule d'un bout à l'autre de la vie. La notion de formation tout au long de la vie souligne l'importance non pas seulement de la formation à l'âge adulte, mais aussi celle de la formation à tous les stades de la vie, et notamment le fait « d'apprendre à ap prendre » dans les établissements scolaires et autres établissements d'enseignement formel - à la fois tout au long de la vie et dans toutes les facettes de la vie. Le capital humain se développe en diverses occasions à travers :

- I'acquisition de connaissances au sein de la famille et dans les différentes structures d'accueil de la petite enfance ;

- les activités formelles d'enseignement et de formation, notamment l'enseignement préscolaire, l'enseignement obligatoire, l'enseignement général ou professionnel après l'obligation scolaire, I'enseignement supérieur, la formation organisée dans le cadre du marché du travail, la formation des adultes, etc. ;

- la formation sur le lieu de travail et les connaissances acquises dans la vie professionnelle, dans le cadre d'activités précises telles que 
la recherche, l'innovation ou la participation à divers réseaux professionnels ;

- les acquis informels, c'est-à-dire sur le tas, dans la vie de tous les jours ou la vie de citoyen.

Cette vision globale du capital humain aide à réfuter la critique selon laquelle la notion de capital humain se déshumanise du fait de l'assimilation des personnes à des mécanismes physiques. A l'inverse, dans son rapport de 1998 sur le capital humain, l'OCDE affirme que la notion de capital humain souligne avec force l'importance des personnes dans les économies fondées sur le savoir et les compétences.

Le capital humain a de multiples facettes. Les qualifications et les compétences peuvent être d'ordre général (savoir lire, écrire, parler) ou extrêmement spécifiques, et plus ou moins adaptées selon le contexte. Les qualifications et les connaissances propres à une entreprise donnée s'acquièrent par la formation sur le tas et en entreprise. Le savoir et les qualifications ainsi acquis sont pour l'essentiel plutôt tacites que codifiés et explicités dans des documents - soit parce qu'ils ne se prêtent pas une codification soit parce que la tâche de la codification n'a simplement pas été entreprise. Plus le savoir est tacite plutôt qu'explicite, plus il est difficile de le transmettre et de le partager. Lundvall et Johnson (1994) classent le savoir en quatre catégories :

1. Le savoir quoi : désigne la connaissance de « faits ».

2. Le savoir pourquoi : désigne la connaissance de principes et de lois auxquels obéissent la nature, l'intelligence humaine et la société.

3. Le savoir comment: désigne les qualifications (autrement dit, les aptitudes à effectuer des tâches).

4. Le savoir qui : désigne l'aptitude à coopérer et à communiquer avec différents types de personnes et de spécialistes.

A la différence du capital physique, le capital humain est le propre des individus2. Le capital humain se développe par son utilisation et l'expérience, à la fois dans l'emploi et en dehors de celui-ci, ainsi que grâce à l'acquisition de connaissances informelles et formelles, mais il tend également à se déprécier s'il n'est pas utilisé. Certaines qualifications vont perdre en importance avec le temps et ce processus explique en partie la baisse du capital humain mesuré (ou du moins du surcroît de revenus du travail lié à un niveau donné de formation) audelà d'un certain âge (Mincer, 1974). Ainsi, on ne saurait concevoir le capital humain comme un ensemble homogène et statique de qualifications ou de compétences acquises une fois pour toutes. Certaines des qualifications et compétences liées au capital humain peuvent être classées comme suit :

1. Communication (dont l'aptitude à utiliser une langue étrangère dans chacune des rubriques indiquées immédiatement ci-dessous)

- écouter

- parler
... et le capital humain présente de multiples aspects...
... comme des qualités allant de l'aptitude à lire et à écrire à la persévérance. 
En pratique, les activités humaines exigent pour la plupart un esprit d'équipe.
Le savoir et les réseaux jouent un rôle sans cesse plus important.
- lire

- écrire

\section{Calcul}

3. Compétences personnelles

- motivation/persévérance

- « apprendre à apprendre » et autodiscipline (y compris les stratégies de formation autodirigées)

- capacité de former des jugements fondés sur un ensemble pertinents de valeurs morales et d'objectifs pour la vie

4. Compétences relationnelles

- esprit d'équipe

- art de diriger

5. Autres compétences et caractéristiques personnelles (utiles dans de nombreux domaines mentionnés ci-dessus)

- aisance dans l'utilisation des technologies de l'information et des communications

- connaissances tacites

- aptitude à résoudre des problèmes (également présente dans d'autres types de compétences)

- qualités physiques et habilité manuelle

Fukuyama (1995) note que la quasi-totalité de l'activité économique est le fait non pas des individus, mais d'organisations, ce qui exige un degré élevé de coopération sociale. Le «capital organisationnel » correspond aux savoirs détenus en commun, à l'esprit d'équipe, aux normes de comportement et aux interactions au sein des organisations ; c'est, dans le domaine de l'organisation, une version du capital social examinée dans le chapitre suivant. Leana et Van Buren (1999) définissent le capital organisationnel « comme une ressource reflétant la nature des relations sociales au sein de l'entreprise. Ce capital se développe dans la mesure où chaque membre, à son niveau, apporte sa contribution à la réalisation de l'objectif collectif dans un climat de confiance mutuelle, ce qui crée de la valeur en facilitant la réussite de l'action de tous ». Les personnels sont encouragés à coopérer lorsque le mode d'organisation limite les comportements opportunistes, favorise la confiance, encourage la franchise dans la communication et promeut l'acceptation de valeurs et d'objectifs communs (voir par exemple, Axelrod, 1984).

Il est de plus en plus admis que la gestion et la mise en commun des connaissances peuvent jouer un rôle primordial dans l'économie désormais dite «nouvelle » en raison de la situation organisationnelle et technologique fondamentalement nouvelle (Lesser, 2000). Les connaissances, l'adaptabilité, la confiance et la constitution de réseaux revêtent une importance accrue alors que l'on recherche des idées et des pratiques nouvelles dans un environnement économique en mutation rapide. De nombreux observateurs ont lié la notion « d'économie nouvelle » à celle «d'économie apprenante », dans laquelle la capacité des réseaux, des entreprises et des personnes à ap prendre, à s'adapter et à communiquer ainsi qu'à mettre leurs connaissances en application est qualitativement plus importante qu'auparavant (OCDE, 2000a). 


\subsection{Comment mesurons-nous le capital humain ?}

Les titres et diplômes sont une solution simple et facile à utiliser pour mesurer les qualifications et les compétences. L'inconvénient est qu'ils ne reflètent pas le capital humain acquis à l'occasion d'activités informelles de formation ou par l'expérience, et qu'il peut être difficile de comparer des diplômes différents (lorsqu'ils sont par exemple délivrés par divers pays). L'autre formule consiste à évaluer, à l'aide de questionnaires, les performances des élèves et les compétences des adultes comme dans le Programme international pour le suivi des acquis des élèves (PISA) ou l'Enquête internationale sur la littératie des adultes (voir le graphique 2.1). Ces évaluations ne portent que sur certains aspects des aptitudes et compétences et se heurtent aux limites propres aux méthodes d'enquête et d'évaluation (concernant, par exemple, la taille des échantillons, la gamme des variables interdépendantes prises en compte ou les pays couverts dans les enquêtes).

Pour établir une estimation de la valeur du capital humain, on a tenté de regrouper les données relatives aux projections des revenus du travail des individus durant leur vie entière, selon le niveau de formation initiale3. Cette démarche occulte l'imp ortance des « connaissances ou qualifications collectives » que possède une organisation ou une autre entité collective 4 . (En principe, les personnes ont peu de chances de monnayer sous forme de salaire l'intégralité de leurs compétences-maison dans la mesure où leur employeur est le seul acheteur potentiel de ces compétences.) Cette méthode de globalisation risque aussi de conduire à négliger les interactions et les retombées 5 engendrées par l'amélioration du capital humain de certains membres. Enfin, il n'est pas aisé de rendre compte dans ces mesures globales des aspects éminemment spécifiques, étroitement liés à la culture, non communicables, tacites et hétérogènes du capital humain.

Ces réserves ind iquent à quel point il faut se montrer prudent dans les estimations de stocks ou de flux d'investissement dans le capital humain, surtout dans les comparaisons internationales. Les calculs à indice unique du capital humain doivent être complétés par des mesures plus spécifiques reposant sur une évaluation directe des connaissances et des qualifications dans les organisations.

\subsection{Comment le capital humain se forme-t-il ?}

Le capital humain se forme à l'occasion non seulement d'activités formelles d'enseignement ou de formation, mais aussi de contacts informels avec autrui ainsi que d'un travail de réflexion personnelle et de formations autodirigées. Les réseaux sociaux et les normes jouent un rôle important tout au long du cycle de vie en favorisant une culture de l'apprentissage. Dans cette section, on passe en revue des données empiriques qui révèlent l'incidence de divers facteurs sur les résultats des activités de formation - ces données portent surtout sur les niveaux d'enseignement primaire et secondaire - et proviennent de nombreux documents couvrant diverses disciplines. D'autres précisions figurent dans les annexes $C$ et $D$.

Nombreux sont ceux qui ont souligné l'importance du contexte culturel dans lequel se déroule l'acquisition de connaissances et l'instruction. Fuller et Clarke (1994), par exemple, passent en revue un certain nombre
Les titres et diplômes sont une solution simple mais insuffisante pour mesurer le capital humain.

Il est difficile de faire la somme des différentes composantes du capital humain...

... et il faut en mesurant le capital humain reconnaître les limites de nombreuses variables représentatives.

L'investissement dans les qualifications s'effectue dans de nombreux cadres et à différents stades de la vie...

... et le contexte culturel exerce une influence sur les activités d'apprentissage. 


\section{Graphique 2.1. Répartition comparative des niveaux de littératie}
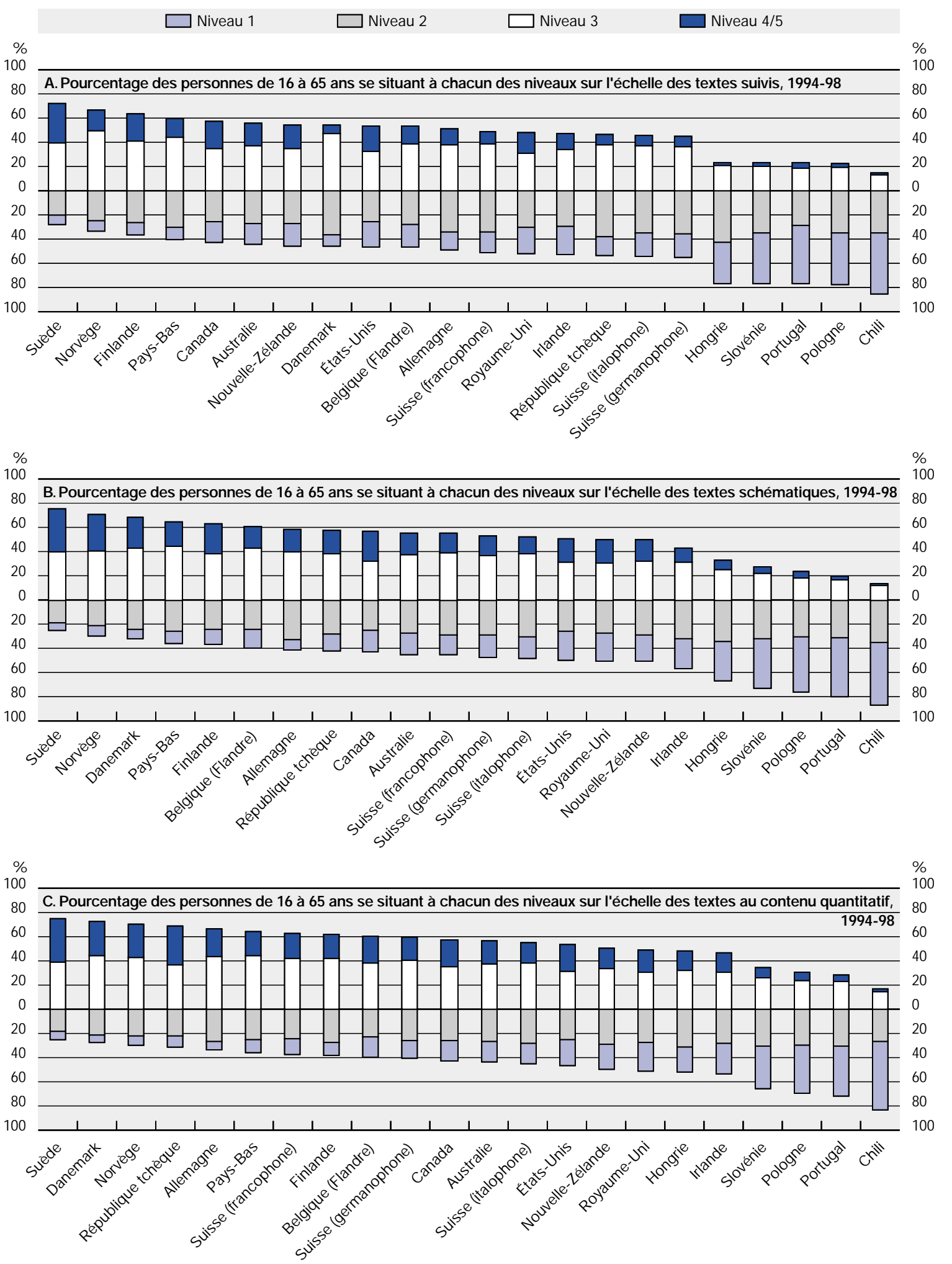

Note: Les pays sont classés selon la proportion des personnes aux niveaux 3 et $4 / 5$.

Source : OCDE (2000), La littératie à l'ère de l'information, Paris. 
de différentes méthodes, dont celles qu'ils qualifient de «mécanique décisionnelle $\gg 6$ et de 《culturalisme en classe $\gg 7$ destinées à évaluer la productivité de l'école. Outre les normes et attitud es propres à leur famille et à leur milieu social, les élèves acquièrent des normes, des habitudes et des valeurs particulières dans le cadre culturel de l'école ou de la classe. Ces normes renforcent ou modifient leur perception à différents égards - mérite, statut, normes de comportement et de participation - de même que leur attitude à l'égard de l'acquisition de connaissances. Ainsi, la signification d'ordre culturel attachée à l'acquisition de connaissances ainsi qu'aux pratiques et outils pédagogiques peut compter tout autant que le contenu matériel des apports scolaires ou que le volume des ressources affectées. Fuller et Clarke affirment que tout en représentant une optique valable, la méthode culturaliste n'a pas produit de données empiriques sur la variabilité transculturelle de la signification attribuée aux outils et aux pratiques d'enseignement pas plus qu'elle n'a réussi à déterminer des moyens d'action susceptibles de dynamiser l'apprentissage dans différents contextes culturels.

Les dépenses publiques d'éducation, tous niveaux confondus, n'ont cessé d'augmenter dans les pays de l'OCDE ces dernières années, ce qui témoigne à la fois de l'accroissement de la fréquentation (surtout au-delà de l'obligation scolaire) et de la progression des dépenses réelles par élève - souvent liée à une diminution de la taille des classes dans les établissements scolaires. Les données donnent à penser que le volume des dépenses exercent effectivement une influence, limitée toutefois, sur les résultats et que leur efficacité est étroitement liée à la façon dont les pratiques pédagogiques, l'organisation de l'école et le soutien des parents se conjuguent avec l'accroissement du financement de l'enseignement (Hanushek et Kim, 1995, Hanushek et Kimko, 2000 et Gundlach, Wossmann et Gmelin, 20008).

Certaines données laisseraient entrevoir un rendement en baisse des dépenses affectées aux activités formelles d'enseignement dans les pays ayant un développement économique relativement élevé (Hanushek et Kim, 1995). Dans les pays en développement, Fuller et Heyneman (1989) ont montré que l'école influe plus que la famille sur les résultats des élèves dans les milieux démunis, ce qui renforce l'argument selon lequel les pouvoirs publics peuvent agir sur le niveau de formation des plus défavorisés. Une importante conclusion se dégageant de la plus grande partie des travaux empiriques menés à ce sujet est qu'en général l'augmentation des dépenses induites par une plus grande fréquentation de l'école (due en particulier à une baisse des sorties prématurées du système scolaire) a peut-être un meilleur rendement (à travers une amélioration des revenus du travail) que l'augmentation des dépenses par élève par niveau et année. Cette remarque semble s'appliquer aussi bien aux États-Unis (Betts et Roemer, 1998) qu'aux pays en développement (Psacharopolous, 1994).

L'accroissement des dépenses d'éducation ces dernières décennies a servi pour une grande part à réduire la taille des classes. II s'agit de savoir si à l'avenir la réduction de la taille des classes sera rentable. Les effets dépendent de l'âge ou des besoins des groupes concernés - ainsi, cette réduction pourrait avoir des effets positifs pour les jeunes enfants (dans les écoles maternelles ou en première année d'en-
Pour améliorer les performances, l'accroissement des dépenses d'éducation doit être complété par d'autres stratégies.

Dans les pays dont le niveau de développement économique est relativement élevé, le rendement des dépenses d'éducation pourrait être en baisse.

Les classes de plus petite taille paraissent effectivement associées à une élévation du niveau, mais l'effet reste limité. 
L'influence respective de la famille et de l'école est débattue.

Les réseaux sociaux sont importants dans la formation. seignement primaire). Des recherches effectuées aux États-Unis et dans d'autres pays de I'OCDE donnent à penser que les classes de plus petite taille obtiennent effectivement des résultats légèrement meilleurs, d'ap rès les résultats des élèves aux évaluations. Toutefois, quels qu'ils soient, les avantages possibles de cette réduction doivent être mis en balance avec les coûts et le rendement d'autres stratégies possibles, telles que le renforcement des efforts pour améliorer la formation et la qualité des enseignants. II se peut qu'une réduction de grande ampleur, ramenant le nombre d'élèves par classe de 25 à 15, par exemple, permette d'améliorer légèrement les résultats, mais il est aussi probable que les coûts induits seront considérables. Dans certaines disciplines, pour certains groupes d'apprenants, et en conjuguant la réduction avec une adaptation particulière des méthodes pédagogiques, on obtiendra vraisemblablement de meilleurs résultats avec des classes moins chargées. D'autres recherches sont nécessaires au sujet de cette possibilité, liée à l'éventuelle utilisation de classes à petits effectifs dans certains cas et de classes à effectifs plus importants dans d'autres cas.

Il est très largement ad mis que le milieu familial et social influe sur l'efficacité de l'école. De nombreuses études mettent en évidence l'importance de facteurs tels que le soutien que les parents accordent à leurs enfants, les aspirations et habitudes de travail qu'ils leur transmettent. Utilisant les données provenant d'un grand nombre de pays participants à la Troisième étude internationale sur les mathématiques et les sciences (TIMSS), Wossmann (2000), par exemple, constate que l'influence exercée par les parents sur la formation de leurs enfants ou l'intérêt qu'ils montrent pour leurs études ainsi que pour leur orientation scolaire a une importante incidence sur les résultats des élèves. Bourdieu (1979) utilise l'expression «capital culturel » pour désigner les habitudes ou pratiques culturelles fondées sur le savoir et les comportements appris au contact de modèles de rôle au sein de la famille et dans d'autres milieux. Le capital culturel représente aussi l'ensemble du patrimoine familial, dont le niveau d'instruction des parents, la classe sociale et les habitudes, normes et pratiques familiales qui influent sur la réussite scolaire. Cinq grandes catégories ont été définies (Kellaghan et al., 1993) : les habitudes de travail de la famille, l'aide aux devoirs et les conseils, l'incitation à réfléchir et à débattre d'idées ou d'événements, les habitudes langagières (possibilités de penser et d'exercer son imagination) et les aspirations et attentes des parents quant aux études de leurs enfants9. Les parents ou les frères et sœurs peuvent aider les plus jeunes à faire leurs devoirs et leur inculquer le goût d'apprendre et la discipline. En outre, plus les attentes des parents (en particulier de la mère) sont fortes, plus la probabilité d'aband on des étud es est faible. Ce sont autant de moyens efficaces d'atténuer les effets négatifs, sur les résultats scolaires des enfants, d'une origine socio-économique modeste ou du manque d'instruction des parents (White et Kaufman, 1997).

Coleman, qui a été le premier à introduire le concept de capital social dans la recherche sur l'enseignement, a souligné l'importance du monde des adultes entourant les jeunes, qui se trouvent « enclavés » au milieu des adultes dont ils sont le plus proches (Coleman, 1988). Le capital social désigne les ressources acquises grâce aux liens sociaux, à l'appartenance à des réseaux et le partage de normes communes. A l'inverse, le capital 
culturel - qui est un aspect du capital social - désigne les ressources propres aux familles, qui permettent aux individus d'atteind re une position sociale particulière (Bourdieu, 1979 ; Bourdieu et Passeron, 1970). Les familles et les élèves ont accès à des niveaux variables de capital financier, humain, culturel et social, mais ce capital à lui seul ne garantit pas un bon niveau de formation. Coleman affirme que le capital social peut favoriser l'acquisition de connaissances à travers l'existence de multiples formes de relations de soutien entre des parents d'enfants fréquentant la même école. II peut s'agir d'aide pour les devoirs, d'activités parascolaires ou encore de la participation directe des parents aux activités de l'école et de l'aide aux familles et aux enfants en difficulté. Putnam (2000a) fournit des données empiriques pour les États-Unis au sujet de l'impact du capital social sur les résultats scolaires des élèves (voir l'annexe $C$ pour plus de détails).

La prudence s'impose lorsqu'on définit les déterminants les plus significatifs des résultats des élèves. A elle seule, l'existence de ressources matérielles ou d'enseignants hautement qualifiés ne suffit pas. Tout dépend de la manière dont les différents acteurs, dont les élèves, les parents, les enseignants et les membres de la communauté scolaire au sens large, interagissent et utilisent les ressources disponibles. A la difficulté de définir les facteurs clés s'ajoute le fait que les résultats varient selon l'aspect des performances qui est mesuré (les incidences varient en fonction de la discipline ou du domaine de compétence) et selon le groupe de population considéré (un soutien ciblé sur tel groupe particulier peut être plus efficace que s'il était accordé à d'autres groupes). En général, il est essentiel de renforcer les partenariats entre les élèves, les parents et les enseignants et de les étayer par des dispositions institutionnelles et financières appropriées.

La plus forte demande de formation liée à l'emploi émane d'un groupe de travailleurs, allant des jeunes aux quadragénaires, ainsi que des personnes dont le niveau de formation est supérieur à la moyenne. D'une façon plus générale, la demande d'activités de formation continue est liée aux habitudes qui ont été acquises et aux possibilités qui ont été offertes à un stade précoce de la vie (OCDE, 1998). La formation pour adultes est en grande partie informelle, expérientielle et imbriquée dans la vie quotidienne et le travail, d'où les difficultés rencontrées pour l'observer et la mesurer. Il est difficile de distinguer et de mesurer les facteurs qui assurent l'efficacité et la qualité des activités informelles de formation. Les données existantes dans ce domaine portent davantage sur les activités d'enseignement formel et de formation continue. En l'occurrence, il semblerait que dans le cas de la formation liée à l'emploi, pour élaborer des programmes qui répond ent aux besoins des différents app renants et leur procurent les qualifications et les connaissances demand ées sur le marché du travail, le mieux est de le faire en partenariat avec les app renants, les prestataires et les employeurs (OCDE et Département de l'É ducation des États-Unis, 1998). Les cadres d'apprentissage formels ou de type scolaire ne garantissent pas nécessairement l'efficacité des résultats puisque la formation s'inscrit dans un contexte différent et qu'il faut assurer une plus grande personnalisation des activités. Si l'on considère les types de formation moins formels et moins liés à l'emploi, une très large palette d'expérience et de cadres sont possibles 10 . Il importe de déterminer les centres d'intérêt des adultes et non pas seulement les qualifications qui leur manquent.
Les partenariats, le financement et des réglementations efficaces jouent un rôle.

Pour être efficace, la formation des adultes doit être soigneusement définie. 
Les réseaux sociaux contribuent à favoriser l'acquisition de connaissances tout au long de la vie.

La répartition est liée à des normes globales et à la qualité du capital humain.

La situation des personnes
défavorisées ne s'est pas
améliorée dans de nombreux pays...
S'il est difficile d'isoler les différents facteurs en jeu, il semble effectivement que des niveauxélevés d'engagement social et de confiance offrent des possibilités d'app rendre dans des cad res informels et formels. Ohsako (1998), citant les résultats de la «International Comparison of Learning and Social Participation by the Eld erly $\gg 11$ (ICLSE - comparaison internationale de l'engagement des personnes âgées dans des activités de formation et dans la vie sociale), fait état d'une corrélation, dans le cas des anciens, entre leur participation à des activités de formation et leur engagement actif dans la vie sociale et collective. Les associations bénévoles peuvent jouer un rôle important en favorisant une culture de l'apprentissage. Ap paremment, une forte interdépendance existe entre le niveau de formation initiale, le niveau de participation à des activités de formation continue et le niveau de confiance et d'engagement dans des activités civiques, ce qui laisse entrevoir un cercle vertueux. Comme on le notera dans le chapitre 3, certains réseaux sociaux peuvent entraver l'ap prentissage, lorsque par exemple le poids des liens familiaux et ethniques limite les possibilités de progrès et de formation personnelle12.

\subsection{Comment le capital humain est-il réparti ?}

La répartition des qualifications et des possibilités de formation pour adultes varie considérablement d'un pays à l'autre $(O C D E$ et Statistique Canada, 2000). Les groupes qui risquent d'être en échec scolaire et par conséquent de se retrouver exclus du marché du travail et de la société sont défavorisés dès le début de la formation initiale. Avec le temps, ce handicap peut s'aggraver à l'école et aboutir à des taux très faibles d'obtention d'un diplôme, de poursuite d'étud es plus poussées et de progression dans l'emploi. Le handicap éducatif a été défini et mesuré de diverses façons, selon que l'on se focalise sur l'individu ou sur des groupes ou que les phénomènes examinés concernent le financement, l'accès, l'obtention de diplômes ou encore le degré de réussite ou de compétence atteint dans la vie de tous les jours. Dans le cadre scolaire, I'une des définitions utilisées porte sur la situation de handicap dans laquelle un enfant se trouve si, en raison de facteurs économiques, culturels ou sociaux, les compétences qu'il possède sont différentes de celles qui sont valorisées par le système scolaire, et qui sont nécessaires pour faciliter son adaptation à l'école et son processus d'apprentissage (Kellaghan, 1999).

Dans la plupart des pays de l'OCDE, on observe une diminution de la proportion d'adultes ne maîtrisant pas les savoirs fondamentaux, c'est-àdire le «lire-écrire-compter », ce qui reflète l'effet décalé dans le temps de l'allongement de la scolarité obligatoire durant la deuxième moitié du 20e siècle. Pourtant, il n'est pas évident que l'éducation ait contribué à réduire l'écart entre les différents groupes sociaux, qu'il s'agisse de l'accès à l'enseignement post-secondaire et supérieur ou de la situation socio-professionnelle relative des différents groupes. Dans certains cas, la progression de la demande d'enseignement tertiaire n'a peut-être pas bénéficié aux personnes issues des milieux sociaux défavorisés et risque même d'avoir aggravé la situation des personnes peu qualifiées vis-à-vis de l'emploi. Des données à l'appui de ces constats figurent dans OCDE (2001). Depuis les années 80 , les inégalités des revenus tirés de l'emploi se sont accentuées dans certains pays de I'OCDE comme les États-Unis, non seulement à l'intérieur des différents niveaux de formation, mais aussi 
entre eux. Cette situation peut s'expliquer notamment par une plus grande disparité de la qualité de la formation initiale, une évolution de la demande d'aptitudes de différents types, que ne mesure pas le niveau de formation initiale, mais aussi d'autres raisons déterminées par le marché ou par les institutions (Levy et Murnane, 1999).

Une étude réalisée par Blossfeld et Shavit (1993) concernant deux cohortes (nées en 1910 et 1960) dans 13 pays13 a montré que le milieu socio-économique a toujours un effet tout à fait déterminant sur le niveau de formation atteint et la participation à des activités d'enseignement dans la plupart de ces pays. Ce constat vaut en particulier dans le supérieur où les obstacles (dus aux coûts, à l'information, à la motivation ou aux aptitudes acquises) à une plus large fréquentation persistent en dépit de l'accroissement des effectifs dans le deuxième cycle du secondaire. Les auteurs ont également constaté que l'incidence de l'origine sociale est plus grande aux niveaux inférieurs de transition (par exemple, au passage du premier au deuxième cycle de l'enseignement secondaire), la sélection sociale intervenant plus tôt. Enfin, les auteurs affirment que la réforme de l'enseignement n'a guère eu d'impact voire aucun sur la diminution des inégalités, excepté peut-être en Suède et aux Pays-Bas. A l'inverse, Blossfeld et Shavit ont pu constater une certaine réduction de l'écart entre les sexes parallèlement au développement de l'enseignement.

Erikson et Jonsson (1996) mettent en relief trois principaux facteurs qui sont à la base de l'inégalité des chances devant les études et des écarts de résultats scolaires dans l'ensemble des pays :

- les différences de possibilités d'apprendre «en dehors de l'école » ;

- les différences de taux de poursuite des études et de possibilités d'intégrer des filières «p restigieuses »; et

- les différences de développement global de l'enseignement.

Erikson et Jonsson attirent l'attention sur le rôle que joue le deuxième de ces trois facteurs dans l'explication des écarts d'un pays à l'autre. Une égalité socio-économique relative conjuguée à une réforme globale de l'enseignement et à l'allongement de la scolarité obligatoire ont contribué à diminuer les disparités au fil des ans. Plus les élèves sont âgés au moment où ils peuvent quitter le système scolaire et où ils sont appelés à faire un choix entre différentes filières d'enseignement, plus les risques d'échec sont faibles.

S'il est vrai que Hanushek et Somers (1999) ne trouvent que peu d'éléments prouvant que les dépenses publiques dans l'ensemble des États-Unis sont liées aux résultats des élèves et, en dernière analyse, à leur devenir sur le marché du travail, d'autres études donnent à penser que des programmes soigneusement conçus et bien ciblés peuvent avoir une incidence positive sur l'acquisition de connaissances et sur les chances dans la vie s'ils répondent aux besoins des apprenants issus des milieux défavorisés (Kellaghan, 1999). Ainsi, l'accueil et l'éducation des jeunes enfants peuvent améliorer le développement cognitif durant la prime enfance et aboutir à une amélioration durable du processus d'ap-
... et en dépit des initiatives prises par les pouvoirs publics en matière d'égalité devant l'enseignement, des inégalités persistent.

Toutefois, des programmes soigneusement conçus et bien ciblés peuvent avoir une incidence positive. 
L'inégalité économique va de pair avec l'inégalité d'accès à l'enseignement et les écarts de niveau de littératie des adultes.

Remédier au handicap peut être le moyen d'élever le niveau global. prentissage et des résultats scolaires (OCDE, 1999a). Par ailleurs, l'impact des interventions ciblées sur les jeunes défavorisés ne saurait s'évaluer uniquement au regard de résultats cognitifs. II convient également de tenir compte des autres avantages possibles de la socialisation et de l'épanouissement personnel liés à ces interventions, même s'ils sont plus difficiles à mesurer.

Dans la population adulte, les personnes peu qualifiées ou ayant un faible niveau d'instruction risquent davantage d'être au chômage ou exclues de la société (Steedman, 1996). Les sociétés qui tendent à être moins égalitaires du point de vue de l'accès à l'enseignement et des niveaux de formation le sont également moins en matière de répartition des revenus. Conjugués, le niveau de formation, le milieu social et l'accès au capital social peuvent influer sur les chances dans la vie. L'Enquête internationale de l'OCDE sur la littératie des adultes (OCDE et Statistique Canada, 2000) révèle :

- de grandes différences dans le niveau général de littératie (qui englobe la compréhension de textes suivis, la compréhension de textes schématiques et la compréhension de textes au contenu quantitatif14) dans l'ensemble des pays ;

- d'importants groupes de population peu qualifiés, même dans les pays où le niveau d'instruction est élevé ;

- l'existence d'un lien étroit entre les niveaux de littératie et le devenir social, professionnel et civique.

Les pays où la fourchette des résultats de littératie est plus étroite au sein de la population en âge de travailler obtiennent en général de meilleurs résultats globaux dans les comparaisons internationales. Ce constat donne à penser que pour relever le niveau de maîtrise des savoirs fondamentaux de l'ensemble de la population et répondre aux besoins futurs de qualifications, il est peut-être essentiel de s'occuper des groupes de population dont le faible niveau de qualifications est lié aux inégalités sociales et éducatives.

Willms (2001) montre qu'il existe un lien étroit entre le contexte familial, éducatif et social des individus d'une part et les résultats scolaires et le niveau de littératie des adultes d'autre part. Cette remarque s'applique aux différents pays et aux différents sous-groupes à l'intérieur des pays qu'il a analysés. II ressort que les variations des résultats de littératie au sein des sous-groupes de la société ou des pays sont plus larges lorsque les parents ont un faible niveau de formation ou niveau social que lorsque ces niveaux sont relativement plus élevés.

Ce point est corroboré par le constat suivant : les pays (notamment les pays nordiques et la République tchèque) qui ont réussi à atteindre des niveaux élevés de littératie des adultes, d'ap rès les critères internationaux, semblent y être parvenus en partie en réduisant les inégalités entre les différents groupes sociaux du point de vue de la maîtrise des savoirs fondamentaux. Les États-Unis se distinguent par un niveau d'instruction globalement élevé et des scores moyens de littératie des adultes, mais ils figurent parmi les pays de I'OCDE affichant la plus forte concentration de faibles 
aptitudes parmi les personnes peu instruites. C'est pourquoi, l'amélioration des compétences de base pourrait, semble-t-il, avoir une incidence importante sur les groupes de population dont le niveau est le plus faible.

\subsection{L'évolution de la demande de capital humain}

Les niveaux de formation (d éfinis par type ou par degré) ou de qualification de la population active peuvent être mal assortis à la demande sur le marché du travail. Une inflation des diplômes se produit lorsque les employeurs relèvent systématiquement le niveau de formation qu'ils exigent sans qu'il y ait un accroissement correspondant de la technicité des emplois.

La progression rapide des niveaux de formation et des niveaux de littératie au cours de la dernière décennie donne à penser qu'il n'y a pas de pénurie de capital humain dans les pays de I'OCDE. De nombreux anaIystes du marché du travail, dont Hartog (1997), observent à la fois en Europe et aux États-Unis ce qu'ils appellent une suréducation, un renforcement de ce phénomène étant possible au fil des ans en Europe. Green, Mclntosh et Vignoles (1999) constatent une certaine progression de la suréducation au Royaume-Uni entre les années 70 et les années 80 , mais n'observent guère d' "inflation des diplômes » généralisée. Toutefois, l'absence d'éléments prouvant une baisse générale ou sur le long terme des taux de rendement de l'enseignement (OCDE, 1998) infirme une hypothèse de «suréducation ». De plus, cette expression est quelque peu trompeuse car elle tend à occulter les fonctions plus générales de la formation, qui est une ressource importante utilisée également en dehors de la vie professionnelle ou comme source de bien-être en soi.

Les employeurs peuvent utiliser les diplômes comme des signaux ou comme des «moyens de sélection » pour repérer les aptitudes innées et la motivation, qui ne sont pas nécessairement le résultat de la formation (Spence, 1973). Autrement dit, les gains associés à ces diplômes peuvent refléter en partie ces aptitudes préalables plutôt que la valeur ajoutée par le diplôme. On parle dans ce cas de «signalisation ». II ne fait guère de doute que ce processus contribue à expliquer les écarts de salaires fondés sur le niveau d'études, mais son importance globale demeure controversée15.

L'analyse des inadéquations entre les emplois et les qualifications, faite au Royaume-Uni à partir des données tirées de l'Enquête internationale sur la littératie des adultes (Green, McIntosh et Vignoles, 1999), conduit à penser que pour un niveau de formation donnée, non seulement les niveaux de compétences inférieurs mais une sous-utilisation des compétences sont pénalisants du point de vue des revenus du travail. Les diplômés universitaires canadiens ayant de faibles niveaux de littératie risquent bien davantage que d'autres diplômés universitaires de faire l'expérience d'un décalage entre les études faites et les emplois proposés (Boothby, 1999).

Les changements structurels observés dans l'économie, parallèlement à la progression des secteurs de hautes technologies et à l'élévation du niveau d'études exigé dans tous les domaines d'activité, entraînent une évolution de la demande de qualifications. Selon Levy et Murnane (1999), l'accroissement tendanciel aux États-Unis de la variation des revenus du

\section{On peut concevoir}

différemment l'inadéquation de la demande et de l'offre de capital humain.

Une certaine « suréducation » existe, en particulier pour l'enseignement supérieur...

... dans le même temps, toutefois, des indices de sousqualification existent.

Les économies à plus forte intensité de savoir exigent des qualifications différentes... 
... ainsi qu'une plus grande

flexibilité et des aptitudes à

travailler en équipe pour étayer les nouvelles formes du capital organisationnel. travail (à l'intérieur des différents niveaux de formation) tient en partie au fait que les compétences polyvalentes - autrement dit, l'aptitude à communiquer, à travailler en équipe et à résoudre des problèmes, qu'exigent les secteurs en expansion des services aux personnes et du marketing - bénéficient d'un avantage salarial accru. Ces compétences polyvalentes ne se reflètent pas dans les niveaux d'études - et donnent lieu à des écarts de rémunération à l'intérieur des différents niveaux. Beaucoup d'ouvrages nouveaux donnent à penser que la disparité grand issante des salaires dans certains pays pourrait s'expliquer par le différentiel de rentabilité de différents types de compétences (par exemple, Carliner, 1996 ; Rivera-batiz, 1994). Si I'on en croit ces ouvrages, dans certains pays, et notamment au Canada, au États-Unis et au Royaume-Uni, il pourrait être plus rentable de maîtriser la compréhension de textes au contenu quantitatif que la compréhension de textes suivis.

Les employeurs exigent des travailleurs qu'ils soient non seulement plus qualifiés mais aussi souples et « aptes à se former ». C'est pourquoi les travailleurs acquièrent une solide formation initiale ainsi que les compétences génériques d'employabilité afin d'améliorer leurs débouchés sur le marché du travail. Une demande plus intense de «partage du savoir » et de capital organisationnel de la part des organisations et des entreprises génère une demande non seulement de qualifications individuelles plus importantes, mais aussi d'efficacité accrue dans la gestion, le travail en équipe et la flexibilité. Le développement des nouvelles formes de capital organisationnel nécessitant davantage de travail en équipe, un contrôle hiérarchique moindre et une plus grande responsabilité individuelle laissent présager de nouveaux profils de compétences et de nouvelles formes de coopération entre les personnes. Les données tirées de deux enquêtes réalisées en Grande-Bretagne en 1986 et 1997 révèlent, dans les recrutements récents des entreprises, une demande accrue d'aptitud es à communiquer, de « compétences sociales » et de capacité à résoud re les problèmes (Green et al., 1997). Dans les secteurs plus traditionnels et moins orientés vers le savoir, on a tendance à demander aux travailleurs d'exécuter des tâches plus étroitement définies, encore que nombre des entreprises y privilégient le travail en équipe et la flexibilité. Des études de l'organisation du lieu de travail et de l'entrep rise effectuées par ailleurs corrob orent ces observations (par exemple, Cappelli et Rogovski, 1994 ; Freeman, Kleiner et Ostrogoff, 1997).

\subsection{Quel est l'impact du capital humain sur le bien-être économique ?}

L'un des moyens de mesurer l'impact économique du capital humain

Le capital humain a une incidence positive sur les revenus du travail, l'emploi et la croissance économique. consiste à calculer la productivité des investissements dans la formation ou leur « taux de rendement » fondé sur les revenus du travail. On peut évaluer le taux de rend ement privé en utilisant les données relatives aux dépenses privées et aux revenus après impôt de toute une vie (voir OCDE, 1998). En principe, ce taux de rendement privé devrait inclure des avantages non financiers, le plaisir d'apprend re et une plus grande satisfaction dans le travail par exemple, qui peuvent découler du fait de posséder un diplôme. Le taux de rendement social devrait englober l'ensemble des coûts et avantages pour le grand public et la société résultant d'un accroissement des investissements dans le capital humain. En pratique, toutefois, le calcul de l'ensemble des coûts et avantages soulève de nombreuses difficultés et les estimations publiées reposent souvent beaucoup sur une série relative- 
ment limitée de facteurs mesurables. II est en particulier difficile de tenir entièrement compte des effets de la formation en cours d'emploi sur les revenus du travail, ou des avantages du capital humain dont bénéficie l'ensemble de la société ou encore des multiples avantages non économiques. Une autre solution consiste à rechercher à partir de statistiques nationales ou régionales des données empiriques prouvant l'impact du stock de capital humain et de son taux d'évolution sur le niveau et le taux de croissance économique. De nombreux ouvrages visent à établir des estimations de ce genre à partir de données à la fois micro et macro-économiques.

\subsubsection{Données micro-économiques sur les revenus du travail et l'emploi}

Une population mieux instruite a plus de chances d'être au travail et si elle est économiquement active elle risque moins d'être au chômage. De meilleurs diplômes appellent par ailleurs des avantages salariaux. Dans certains pays, ces avantages sont très importants, reflétant un éventail des salaires plus grand sur le marché du travail et vraisemblablement une rentabilité plus grande de qualifications particulières. Des données micro-économiques montrent qu'une année supplémentaire d'études se traduit par une rémunération en moyenne supérieure de 5 à 15 pour cent (Krueger et Lindahl, 1999).

Des recherches dans un domaine connexe ont cond uit à examiner l'incidence de différents types de littératie des adultes sur les revenus du travail. D'après des données communiquées par les États-Unis, la rentabilité de la littératie augmente avec la technicité des emplois (Raudenbush et Kasim, 1998). Dans une enquête longitudinale britannique, Bynner et al. (2001) font état d'une rentabilité salariale de 8-10\% pour le niveau 1 des aptitudes en calcul, tel qu'il est défini par le Q ualification et Curriculum Authority (QCA) du Royaume-Uni16. Selon les données recueillies lors de l'Enquête internationale sur la littératie des adultes (OCDE et Statistique Canada, 2000), l'instruction, la littératie, l'expérience, le sexe, le niveau d'études des parents et l'utilisation de la langue maternelle - autant de facteurs qui sont liés au capital humain - entrent pour 20 et $50 \%$ dans la variation totale des revenus du travail (op. cit., graphique 4.10). La corrélation entre le degré de littératie et le niveau d'études d'une part et les revenus du travail d'autre part varie selon les pays. Cette corrélation implique des effets variables, d'ordre national et institutionnel, sur la façon dont la maîtrise des savoirs fondamentaux est rémunérée sur les marchés nationaux du travail.

\subsubsection{Les données macro-économiques sur la croissance du PIB}

Un modèle courant de croissance économique fondé sur le capital physique et le travail a été mis au point par Solow (1956). Dans ce modèle, la production augmente plus rapidement que les deux principaux facteurs économiques - le capital et le travail - ce qui laisse un facteur « résiduel » représentant le progrès technique. Les détracteurs de ce type d'analyse causale de la croissance font observer qu'il est périlleux de mesurer la qualité du stock de capital humain. De plus, comme le font remarquer Barro et Sala-I-Martin (1995), les résultats d'une analyse causale de la croissance ne permettent pas d'expliquer pourquoi les données observées vont à l'encontre de ce qu'on pourrait croire17.
Il existe une corrélation positive entre la formation et I'emploi, la formation et les revenus.

Indépendamment des qualifications, la littératie des adultes a une incidence importante sur les revenus du travail.

II est difficile d'expliquer le rôle que joue le capital humain dans la croissance. 
Les retombées du savoir sont d'une importance primordiale...
... en particulier dans la relation entre l'enseignement supérieur d'une part et la recherche appliquée et le développement d'autre part.
Une autre solution pour mesurer l'impact de divers facteurs sur la croissance du PIB consiste à utiliser des analyses de régression internationales comprenant des variables pour le capital humain, le niveau de formation, le niveau de revenu et, dans certains cas, des variables indirectes pour divers facteurs d'ordre social et institutionnel (Barro, 2001). Toutefois, le choix des pays, les variables retenues, le choix des moments considérés et les caractéristiques des modèles 18 peuvent entraîner de grandes différences dans les résultats obtenus. Certaines analyses portent à la fois sur des pays en développement et des pays développés. De ce fait, les tests statistiques employés sont plus puissants en raison de la variation plus grande des déterminants de la croissance. Dans ce cas, I'hypothèse implicite est que certains déterminants de la croissance sont communs aux pays en développement et aux pays développés, même s'ils sont quantitativement différents.

Dans les modèles antérieurs de croissance économique, les différents niveaux d'études et la qualité de la formation n'intervenaient pas explicitement et il n'était pas tenu compte des possibilités qu'offre le capital humain de générer « des externalités ou des retombées » (à travers, par exemple, son incidence sur la productivité d'autres facteurs). Avec le développement des modèles dits de la «nouvelle croissance » (Lucas, 1988 ; Romer, 1990b; Barro et Sala-I-Martin, 1995), le rôle de l'instruction et de l'acquisition de connaissances dans la production de nouvelles technologies et d'innovations a bénéficié d'une plus grande attention. Les conceptions et les idées nouvelles, fruits de la recherche et du développement produits par les secteurs à forte intensité de savoir, augmentent la productivité de l'investissement dans le capital physique, dans d'autres secteurs et d'autres régions. L'évolution technique générée de l'intérieur, l'accroissement des rendements d'échelle, le savoir-faire acquis à l'occasion d'une production à forte intensité de technologie peuvent alimenter l'accroissement de la production. Ainsi, un secteur de pointe, en expansion et travaillant à l'exportation peut avoir un effet démultiplicateur sur le savoir et l'innovation dans l'ensemble de l'économie à travers la mobilité des compétences et des entrepreneurs et la diffusion des nouvelles technologies et des nouveaux produits. Le stock initial de capital humain constitué au cours d'une période antérieure peut générer de l'innovation et des effets en aval sous la forme de « retombées » ou d' « externalités » positives affectant d'autres entreprises et même d'autres régions ou pays (Acemoglu, 1996). Une partie du stock initial de capital humain renvoie à des connaissances scientifiques fondamentales et appliquées acquises au cours des études supérieures. Harberger (1998) établit une distinction entre l'effet « levain » et l'effet «champignon » sur le facteur résiduel dans les modèles de croissance économique. Le savoir et le capital humain peuvent servir de levain et par-là même accroître la productivité d'une façon relativement uniforme dans l'ensemble de l'économie alors que d'autres facteurs tels que les avancées technologiques ou les découvertes surviennent de façon soudaine et entraînent un accroissement de la productivité plus spectaculaire dans certains secteurs que dans d'autres.

L'enseignement supérieur joue un rôle important dans le développement de la recherche innovante et dans l'acquisition des compétences nécessaires pour ad opter cette recherche. C'est pourquoi, certaines théories sur «la nouvelle croissance » ont visé à construire un modèle plus complexe qui rende compte de la formation du capital humain en don- 
nant une importance primordiale non seulement à l'éducation en tant que telle mais à ses sous-produits, notamment la recherche et l'innovation. Lorsque, par exemple, les dépenses de recherche-développement sont incluses dans les modèles de croissance, l'effet indépendant des études s'en trouve, semble-t-il, réduit. En prenant le rapport entre les dépenses de R-D et le PIB comme approximation du savoir-faire technologique, Nonneman et Vanhoudt (1996) constatent qu'une partie de la croissance attribuée à la formation initiale était en fait associée à des dépenses de R-D.

Les études consacrées à l'incidence de l'instruction sur la croissance économique ont souvent été peu concluantes. Cette situation pourrait en partie être liée à la mauvaise qualité des données et en partie à la difficulté rencontrée pour repérer les interactions complexes à travers lesquelles le capital humain joue un rôle dans le processus de croissance. Pritchett (1999) affirme que la progression des effectifs scolarisés ou du niveau de formation n'a eu aucune incidence positive significative sur le taux d'accroissement de la productivité ou le taux de croissance économique ${ }^{19}$. Parmi les mesures indirectes du capital humain, généralement utilisées, peuvent être par exemple cités : le taux brut de scolarisation (notamment le pourcentage d'inscrits dans l'enseignement secondaire) ; le nombre moyen d'années d'étud es effectuées par la population adulte ; les estimations de la proportion de la population active ou de la population adulte ayant suivi un enseignement primaire, secondaire ou supérieur ; ou des estimations de la qualité de l'enseignement, établies à partir des résultats des élèves aux examens ou des enquêtes sur la littératie des adultes. L'analyse de l'impact de ces variables ind irectes sur la croissance économique comporte un certain nombre d'écueils et de difficultés qu'il est difficile de surmonter:

i. parfois on ne sait pas au juste dans quel sens est orienté le lien de causalité ;

ii. le rôle du capital humain peut être masqué par son interaction avec d'autres facteurs : I'adaptation des nouvelles technologies et de l'organisation du travail et une répartition plus efficace du capital physique ;

iii. le niveau de formation est une variable indirecte brute du rôle du savoir et des qualifications ;

iv. des erreurs de mesure dues à une classification des niveaux de formation inap prop riée ou non comparable selon les normes internationales ;

v. les pays atypiques ou « en marge » peuvent fausser les résultats (Temple, 2001).

Le cas mentionné en ii) ci-dessus se produit, par exemple, lorsque les modèles ne reflètent pas la façon dont certains pays, dotés d'un faible stock initial de capital humain au début des années 60, ont peut-être eu davantage de possibilités d'accélérer leur croissance en important et en appliquant des technologies mises au point à l'étranger. Par ailleurs, les pays qui ont un faible niveau initial de revenu, mais un stock initial élevé de capital humain (ou une masse critique de diplômés de l'enseignement supérieur) peuvent
L'ambiguïté des résultats des recherches antérieures consacrées à l'enseignement et à la croissance peut être liée à la mauvaise qualité des données. 
Les mesures du capital humain fondées sur une évaluation directe des qualifications donnent des résultats plus positifs...

... et les travaux empiriques récents ont produit des estimations plus fiables de l'impact du capital humain sur la croissance économique. tirer parti d'un processus de rattrapage ou de convergence en adoptant ou en ap pliquant des technologies importées.

Un exemple du cas cité en iv) ci-dessus est donné par Steedman (1996) qui signale des incohérences dans la présentation et la classification des données sur les niveaux de formation dans la Classification internationale type de l'enseignement (CITE). Krueger et Lindahl (1999) estiment également qu'une erreur de mesure dans les principales sources de données internationales utilisées peut être à l'origine de ces résultats, en particulier en ce qui concerne les résultats négatifs obtenus pour le niveau de formation des femmes20. Ce dernier résultat est difficile à rapprocher de l'idée selon laquelle l'instruction des filles et des femmes contribue fortement à la croissance économique et au bien-être des pays développés comme des pays en développement 21 .

L'un des inconvénients de la plupart des recherches internationales est qu'il existe vraisemblablement entre les pays d'importantes différences concernant la nature et la qualité de la scolarité, qui pourraient diminuer I'utilité des comparaisons internationales (Temple, 2001). Hanushek et Kimko (2000) et Barro (2001), à l'aide de données sur les tests internationaux des capacités cognitives en mathématiques et en sciences, évaluent la qualité de différents groupes de la population active adulte. Ils ont constaté que les mesures fondées sur la qualité de l'instruction expliquent beaucoup mieux la croissance économique dans différents pays que le simple nombre d'années d'études.

Des travaux plus récents effectués par de la Fuente et Domenech (2000) ainsi que par Bassanini et Scarpetta (2001) ont renforcé les raisons de penser que les erreurs de mesure jouent un rôle important en la matière. De la Fuente et Domenech (2000) ont examiné la série de données22 de Barro-Lee ainsi que des données d'autres sources, notamment celles de Nehru, Swanson et Dubey (1995). Examinant la cohérence chronologique de ces données et les comparant à des données plus récentes provenant de la base des indicateurs de l'enseignement de l'OCDE, ces auteurs ont constaté de très sérieux problèmes de mesure. Ils se sont ensuite employés à améliorer la série de données en utilisant les sources nationales et internationales disponibles, en veillant à assurer la cohérence dans le temps ainsi que la concordance avec les données récemment recueillies par I'OCDE sur les niveaux de formation. En utilisant cette série de données, ils ont constaté que le capital humain a effectivement un impact considérable et positif sur la croissance du PIB ou du revenu par habitant. Ces résultats sont intéressants car ils portent sur un échantillon limité de pays (principalement des pays Membres de I'OCDE) alors que dans la plupart des autres études, on a obtenu des résultats significatifs pour un échantillon associant des pays à revenu élevé et à faible revenu ; lorsque les analyses effectuées à partir de nombreuses études ont porté uniquement sur les pays de l'OCDE, elles ont fréquemment révélé l'absence d'incidence sensible du capital humain sur la croissance (voir Barro, 2001).

L'OCDE a utilisé les données mises au point par de la Fuente et Domenech pour d'autres travaux consacrés aux disparités récentes des taux de croissance économique. Ces travaux révèlent que «l'amélioration du capital humain a été l'un des principaux facteurs du processus de crois- 
sance des dernières années dans tous les pays de l'OCDE, mais plus particulièrement en Allemagne (principalement dans les années 80 ), en Italie, en Grèce, aux Pays-Bas (principalement dans les années 80) et en Espagne où l'accroissement du capital humain a rep résenté une accélération de la croissance de plus d'un demi-point de pourcentage par rapport à la décennie précédente » $(O C D E, 2000 b)$. Pour les pays de l'OCDE dans leur ensemble, il s'ensuit que chaque année sup plémentaire d'études à plein temps (correspondant à une augmentation du capital humain de $10 \%$ environ) est associée à un accroissement de la production par habitant d'environ $6 \%$ (cette estimation est basée sur la spécification que nous avons sélectionnée. Une autre spécification donne le chiffre de 4-7\%).

Dans la mesure où les pays de l'OCDE ont pour la plupart instauré une scolarisation universelle jusqu'à la fin de l'enseignement obligatoire, voire du deuxième cycle du secondaire, il est particulièrement intéressant d'examiner le rôle de stimulateur de la croissance que joue l'enseignement supérieur ou post-obligatoire. Les preuves ne sont pas très probantes dans la mesure où l'on ne dispose pas en général de séries de données chronologiques adaptées. Toutefois, dans une étude, Gemmell (1996), utilisant un indice de la formation de la main-d'œuvre (nombre de travailleurs ayant suivi un enseignement primaire, secondaire et supérieur), examine les effets des trois niveaux d'enseignement dans les pays en dévelop pement et dans les pays de l'OCDE sur la période 1960-1985. En ventilant les échantillons nationaux par niveau de revenu, il constate que, toutes choses égales d'ailleurs, l'enseignement supérieur semble plus important pour la croissance économique dans les pays de I'OCDE, alors que dans les pays en développement, c'est l'enseignement primaire et second aire. Gemmell (1995) a obtenu des résultats analogues à l'aide de données sur la scolarisation. Barro et Sala-I-Martin (1995) ont obtenu des résultats similaires en utilisant les niveaux d'éducation23. On a observé une association positive et significative entre le niveau initial et aussi le développement ultérieur de l'enseignement tertiaire et la progression du revenu par tête dans les pays de I'OCDE.

Le fait que l'enseignement supérieur puisse avoir d'importantes répercussions sur la productivité peut expliquer la différence de résultats obtenus à partir des études micro et macro-économiques des revenus. A l'échelon micro-économique, l'enseignement supérieur, comparé à l'enseignement secondaire du deuxième cycle, a, selon les estimations, une rentabilité qui semble modeste $(O C D E, 1998)$. Toutefois, au niveau macroéconomique, on peut mieux saisir le rôle de l'enseignement supérieur dans la production d'effets indirects et de retombées, ce qui se traduit par un impact plus important.

Lorsqu'on évalue l'impact de l'enseignement supérieur sur la productivité, on peut prendre en considération deux autres aspects : la part respective des différents domaines d'études dans cet impact et la proportion de diplômés de l'enseignement supérieur entrant dans des secteurs de l'économie dont la contribution au PIB n'est pas évaluée correctement (l'administration ou les services publics par exemple). En analysant l'impact du capital humain sur l'accroissement de la productivité de la main-d'œuvre pour les pays de l'OCDE entre 1950 et 1988, Gittleman et Wolff (1995) ont constaté que le nombre d'ingénieurs et de scientifiques par habitant a un impact positif appréciable sur la productivité.
L'enseignement supérieur importe tout particulièrement dans les pays de I'OCDE.

Le domaine d'études dans l'enseignement supérieur est également un aspect pertinent. 
Le capital humain est un facteur d'égalités de revenu.

En résumé, des recherches récentes laissent entrevoir une incidence positive du capital humain sur la croissance économique.

Les effets sociaux positifs de l'instruction l'emportent sur les effets négatifs...
Le capital humain influe également sur l'inégalité des revenus. Ce type d'inégalité, qui s'est en général accentué dans de nombreux pays de I'OCDE depuis le milieu des années 80 , a été rattaché à de multiples facteurs. Une progression des inégalités entre les ménages selon le type d'emploi (temps partiel, emploi temporaire, etc.) a été associée à l'accroissement du pourcentage de ménages avec ou sans travail (OCDE et Statistique Canada, 2000). Alesina et Rod rik (1992) révèlent l'existence d'un lien entre l'instruction et la répartition des revenus, le rapport de cause à effet jouant dans les deux sens. Les données provenant de l'Enquête internationale sur la littératie des adultes (OCDE et Statistique Canada, 2000) révèlent une forte corrélation à l'échelon national entre le niveau des inégalités de revenu et les inégalités dans la répartition des niveaux de littératie (compréhension de textes suivis) - ce qui implique que plus le capital humain est également réparti, moins grandes sont les différences de revenus.

Une idée généralement favorable de l'incidence du capital humain se dégage d'une étude effectuée par Temple (2001) dans laquelle ce dernier conclut :

Au cours des dix dernières années, les chercheurs travaillant sur la croissance sont passés de la détermination des effets assez spectaculaires de la formation à la mise en doute de l'existence même de ces effets. Les recherches plus récentes se situent quelque part entre ces deux extrêmes, mais penchent peut-être davantage du côté des conclusions initiales selon lesquelles la formation a un impact majeur. Un examen des études n'ayant décelé aucun effet nous a fourni quelques bonnes raisons (erreur de mesure, pays aty piques, spécifications incorrectes) de douter de ces résultats. Le bilan des données récentes donne à penser que les effets de la formation sur la productivité sont au moins aussi importants que ceux recensés par les économistes spécialistes du travail.

\subsection{Quelle est l'incidence du capital humain sur tous les aspects du bien-être?}

Un certain nombre de méthodes, décrites ci-dessous, ont été élaborées pour mesurer les « avantages sociaux » de l'instruction et de l'acquisition de connaissances. Les éléments d'information passés en revue dans cette section portent principalement sur les effets mesurés de la formation initiale, dans des domaines tels que la santé, l'assurance sociale, l'art d'être parent, la délinquance et le bien-être personnel ou subjectif. On peut induire certains des effets de la formation sur la santé et le comportement social en favorisant des habitudes, des caractéristiques et des attitudes propices à la création d'emplois, à la productivité, au bien-être personnel, à des choix positifs d'ordre temporel et à l'auto-discipline. Certaines de ces caractéristiques ainsi que les aptitudes et atouts innés sont acquis en dehors de l'enseignement formel mais sont étroitement liés à l'école.

Les compétences humaines peuvent être mises au service d'objectifs destructeurs comme la délinquance. La recherche de certaines formes de capital humain peut exclure la réalisation d'autres objectifs souhaitables et compromettre les relations sociales et le bien-être personnel. Ainsi, une polarisation excessive durant la prime enfance sur les aptitudes cognitives par rap port à la socialisation et au jeu peut ne pas être la démarche la plus adaptée aux besoins de développement des enfants. Comme pour le capital social, ces effets négatifs possibles sont l'exception. 
Un certain nombre d'importantes techniques de mesure ont été élaborées pour chiffrer certains avantages sociaux de l'éducation, notamment la santé. Les travaux réalisés par Wolfe et Haveman (2001), McMahon (2001)24 et Wolfe et Zuvekas (1997) fournissent une palette de techniques permettant d'établir des estimations monétaires des avantages sociaux découlant de l'instruction. Ces techniques reposent sur le calcul du coût «d'achat » des mêmes effets par d'autres moyens possibles25. Behrman et Stacey (1997) résument les travaux consacrés à diverses sources d'effets sociaux plus larges, en se fondant principalement sur des données recueillies aux États-Unis. D'autres éléments d'information sur les avantages sociaux plus vastes de l'instruction ont été relevés dans des rapports récents au Royaume-Uni26.

Des recherches dans lesquelles on a neutralisé les variables de revenu, de race, de situation sociale et autres ont révélé qu'une corrélation tend à exister entre l'instruction et une amélioration de la santé, une baisse de la délinquance, la participation aux activités politiques et collectives et la cohésion sociale. II faut cependant interp réter ces résultats avec soin car on ne sait pas au juste dans quel sens s'oriente le lien de causalité, et d'autres facteurs dont on n'a pas tenu compte peuvent agir. Toutefois, Wolfe et Haveman (2001) ainsi que McMahon (2001) arrivent à la conclusion que les avantages sociaux de l'instruction sont grands - peut-être plus que ne le sont les effets directs macro-économiques et du marché du travail.

L'un des avantages les plus manifestes de l'instruction est l'amélioration de la santé. Plus le niveau de formation est élevé, meilleure est l'hygiène de vie. Les personnes mieux instruites risquent moins de fumer ou de boire beaucoup. D'après les estimations, une année supplémentaire d'études diminue la consommation moyenne quotidienne de cigarettes de 1.6 pour les hommes et de 1.1 pour les femmes (Wolfe et Haveman, 2001). La surcharge pondérale risque également moins de menacer les personnes mieux instruites qui, par ailleurs, ont tendance à faire plus d'exercice par semaine que les personnes moins instruites - environ 17 minutes par année supplémentaire d'études (Kenkel, 1991). Les avantages de l'instruction du point de vue de la santé peuvent être dus en partie aux choix professionnels (choix de métiers dans lesquels les risques professionnels sont relativement moindres) ou aux choix relatifs au lieu de vie (dans des zones moins polluées). Les personnes plus instruites seront probablement plus à même d'identifier les informations pertinentes pour leur santé et de les utiliser de manière à améliorer leur santé (Kenkel, 1991). A partir de données sur les États-Unis, Kenkel démontre cependant que l'essentiel de la variation des résultats en matière de santé ne peut s'expliquer par des différences de connaissances dans le domaine de la santé. Apparemment, l'instruction a un effet sur la santé indépendamment du revenu, de la race, de l'origine sociale et d'autres facteurs.

Un niveau de formation plus élevé est associé à une moindre probabilité de bénéficier de transferts sociaux (Wolfe et Haveman, 2001). Des analyses récentes ont montré que lorsque le niveau d'instruction des mères est plus élevé, la probabilité que leurs filles choisissent de bénéficier de l'aide sociale, si elles y ont droit, est moindre. Des études effectuées sur des demandeurs d'allocations d'incapacité révèlent également qu'un niveau d'instruction plus élevé diminue la probabilité de percevoir ce type d'aide. Des travailleurs plus instruits ont également tendance à être
... et les effets « indirects » de l'éducation sur la croissance économique à travers les avantages sociaux peuvent être aussi grands que ses effets directs.

Les personnes mieux instruites sont en général en meilleure santé...

... ont moins recours aux prestations sociales... 
... et transmettent les

bénéfices de la formation aux générations suivantes.
L'instruction améliore la recherche d'un emploi, produit des consommateurs plus efficients...

... et est liée à la participation à la vie civique.

Une corrélation existe aussi entre l'instruction et le recul de la délinquance... moins touchés par le chômage et à être mieux rémunérés, ce dont la société profite à travers les impôts27.

Le niveau d'instruction d'une génération a des effets positifs sur celui de la génération suivante. Les enfants dont les parents ont atteint le deuxième cycle du secondaire ont plus de chances d'aller eux-mêmes au bout de leurs études secondaires que les enfants dont les parents sont moins instruits (Sandefur, McLanahan et Wojtkiewicz, 1989). Les enfants dont les parents ont fait de meilleures études ont un développement cognitif plus élevé et de plus grandes possibilités de revenus futurs. On observe également des retombées à l'échelon de la collectivité en ce sens que le fait de vivre au sein d'une population dont le niveau de formation est relativement élevé augmente la probabilité que les enfants vivant dans cet environnement aillent au bout de leurs études secondaires, toutes choses égales d'ailleurs (Wolfe et Haveman, 2001).

L'instruction exerce une influence positive sur la recherche d'un emploi, probablement du fait que les personnes instruites savent utiliser l'information ainsi que les réseaux d'accès à l'information et nouer des relations importantes. Les personnes plus instruites sont apparemment aussi des consommateurs plus efficients (Rizzo et Zeckhauser, 1992).

Le niveau de formation est l'une des principales variables prédictives de nomb reuses formes d'engagement politique et social. Verba, Schlozman et Brady (1995) ont constaté, toutes choses égales d'ailleurs, que la participation à la vie politique augmente en même temps que l'instruction. De plus, il existe une corrélation positive entre le niveau de littératie des adultes et leur participation à des activités bénévoles associatives dans de nombreux pays de l'OCDE (OCDE et Statistique Canada, 2000). Bynner et al. (2001), se fondant sur des données relatives au Royaume-Uni, signalent que les « compétences sociales » seraient d'autant plus grandes que le niveau de formation est élevé. II s'agit des compétences d'organisation et de conseil, qui toutes sont de nature à améliorer la qualité de l'engagement dans la vie civique. Ces auteurs font également état d'une plus grande tolérance à l'égard de la diversité, adhésion au principe de l'égalité des chances et d'une résistance à la désaffection pour la vie politique.

Parfois, les résultats sont plus ambigus : Helliwell et Putnam (1999b) ont constaté qu'une progression du niveau moyen d'instruction entraîne une augmentation des niveaux de confiance, mais non une réduction des niveaux de participation à la vie politique, tandis que Nie, Junn et Stehlik-Barry (1996) affirment que ce sont les niveaux d'instruction plutôt relatifs qu'absolus qui déterminent essentiellement la participation à la vie civique.

L'instruction diminue le risque de délinquance en aidant à socialiser les jeunes qui demeurent scolarisés. Wolfe et Haveman (2001) constatent que les variables liées au capital humain environnant ont un effet positif sur les comportements antisociaux. Bien qu'il faille du temps pour que les effets de ces avantages sociaux se fassent tous sentir, la société peut tirer parti d'un investissement dans la formation en dépensant moins pour la protection sociale, la prévention de la délinquance et la mise en application de la loi. 
Des éléments prouvent également l'existence d'un lien positif entre le temps et les sommes consacrés aux œuvres charitables et le nombre d'années de scolarité. Ainsi, une étude montre que par rap port aux diplômés de l'enseignement secondaire, les diplômés de l'université dédient près de deux fois plus d'heures au bénévolat et font des dons représentant une part de leur revenu supérieure de 50 pour cent (Hodgkinson et Weitzman, 1998). Au Royaume-Uni, les données recueillies à I'occasion de la National Child Development Study (NCDS) révèlent une forte corrélation entre le niveau de formation et l'adhésion à des organisations politiques, à des groupes de défense de l'environnement, à des associations féministes, à des groupes caritatifs, à des associations de riverains et de parents d'élèves (Schuller et al., 2001). Bynner et al. (2001) signalent qu'au Royaume-Uni les diplômés de l'enseignement supérieur ont trois fois plus de chances d'être des membres actifs d'une organisation bénévole que les personnes qui n'ont pas terminé leurs études secondaires (c'est-à-dire qui n'ont pas obtenu de diplôme de fin d'études secondaires) et environ deux fois plus que les personnes qui ont achevé leurs études secondaires.

Les éléments d'information donnent à penser que l'instruction a des effets positifs, à la fois immédiats et à long terme, sur le bonheur déclaré par les intéressés eux-mêmes. Blanchflower et Oswald (2000) font état d'estimations des «équations du bonheur » dans des analyses de régression qui relient le bien-être déclaré à diverses caractéristiques individuelles. Ils constatent que le niveau d'instruction est associé à un bonheur plus grand même lorsqu'on neutralise la variable du revenu familial. Il est possible que le niveau d'instruction d'un individu ait un impact positif sur le bonheur des autres; dans ce cas, il se peut que les individus égoïstes tendent à sous-investir dans la formation d'un point de vue social. Inversement, l'instruction peut avoir une incidence sur le bonheur car elle influe sur la perception que chacun a de sa position par rapport aux autres ; en pareil cas, les résultats de Blanchflower et Oswald pourraient exagérer l'effet sur le bien-être d'un développement des activités éducatives proposées. Toutefois, Putnam (2001), se basant sur des données relatives aux différents États des États-Unis, constate que le niveau personnel d'instruction et le niveau moyen de formation dans le comté ont des effets positifs sur le bonheur.

\subsection{Conclusion}

L'instruction, la formation et l'acquisition de connaissances peuvent jouer un rôle important en jetant les bases de la croissance économique, de la cohésion sociale et de l'épanouissement personnel. II faut du temps pour que l'investissement dans le capital humain se développe et rapporte. Pour autant que ces effets puissent être mesurés et comparés, certaines études donnent à penser que l'incidence sociale de la formation (sur la santé, la délinquance, la cohésion sociale) pourrait être aussi prononcée sinon plus que son incidence sur la productivité économique. Toutefois, la corrélation constatée entre les indicateurs globaux de la scolarisation et divers résultats économiques et sociaux ne donne aucune indication sur l'orientation qu'il convient de donner à l'enseignement formel, si ce n'est qu'il est profitable d'apprendre davantage. Des synergies et des complémentarités existent entre l'acquisition de connaissances et d'autres aspects de l'environnement
... ainsi qu'entre l'instruction et le volontariat et le don.

Les ouvrages sur la relation entre l'instruction et le bien-être subjectif et le bonheur se multiplient.

Un surcroît de formation a un rendement non négligeable... 
... et la qualité et la répartition des possibilités de formation sont importantes.

Les mesures du capital humain doivent porter sur les qualifications effectives mais aussi sur d'autres éléments tels que le temps passé dans le système éducatif. social, institutionnel et juridique. Les qualifications et les compétences peuvent avoir une influence indirecte en renforçant les effets d'autres facteurs. L'enseignement supérieur, en partenariat avec des intérêts publics et privés, peut jouer un rôle fond amental en stimulant la recherche et l'innovation propices à une accélération de la croissance du revenu national.

Ce chapitre a mis en évidence une interaction entre le capital humain et le capital social, qui pourrait être vigoureuse. L'acquisition de connaissances et la préparation à la formation qui se font au sein des familles et dans les environnements locaux constituent un socle important en vue du développement permanent du capital humain à travers le système éducatif proprement dit et la formation suivie tout au long de la vie adulte. Les réseaux sociaux et les organisations ap prenantes peuvent également stimuler l'acquisition informelle de connaissances « sur le tas » et dans la vie quotidienne. Toutefois, les liens entre le capital humain et le capital social ne sont pas automatiques.

Un accroissement du capital humain global doit être également complété par des stratégies axées sur:

1. la qualité de l'investissement dans le capital humain et l'adéquation des qualifications avec les exigences sociales et économiques ;

2. la répartition des possibilités de formation au sein des pays, étant donné la relation entre les inégalités et les performances globales; et

3. le risque de sous-investissement du marché en raison des caractéristiques de «bien public » ou d'« externalité » propres au capital humain.

Enfin, les décideurs étant de plus en plus d'accord sur l'importance de la formation tout au long de la vie et dans toutes les facettes de la vie (cette dernière caractéristique impliquant un large éventail d'environnements d'apprentissage), on commence à prendre conscience des problèmes liés à une focalisation excessive sur des interprétations et des mesures étroites du capital humain. Les mesures fondées sur des résultats, notamment les enquêtes sur les capacités de lecture, d'écriture et de calcul, sont un moyen très important de mesurer directement le capital humain. Étant donné la complémentarité des avantages et des inconvénients des mesures classiques du capital humain, fondées sur les moyens mis en œuvre, et des mesures directes, basées sur les enquêtes, toute stratégie globale de mesure devra recourir à ces deux ap proches. 


\section{Notes}

1. Selon la définition d'Inglehart (1997), la culture est « un système d'attitudes, de valeurs et de connaissances commun à un grand nombre au sein d'une société et transmis de génération en génération » (page 15). La culture est acquise et évolue relativement lentement. Selon Inglehart, la culture a plus de chances d'être modifiée par l'arrivée de jeunes générations que par une réorientation des valeurs et des attitudes de personnes qui ont assimilé des croyances et des valeurs fondamentales dès leur petite enfance.

2. Le capital humain et le capital physique présentent des caractéristiques communes en ce sens que le premier i) comporte une dimension temporelle dans laquelle des investissements sont réalisés et des avantages obtenus et ii) implique de renoncer à des avantages à court terme. Mais il diffère du capital physique dans la mesure où les règles habituelles d'aliénabilité et de souveraineté du consommateur (en vertu desquelles les consommateurs ont la possibilité d'acheter et de « posséder » un bien externe) ne s'y appliquent pas. Par définition, le capital humain réside dans les individus, et les services qu'il produit peuvent faire l'objet d'échanges marchands, mais, à strictement parler, la propriété de ce capital ne peut faire l'objet d'une cession ni être vendue, sauf dans le cas de l'esclavage humain.

3. Pour calculer la valeur du stock de capital humain, on retranche de la valeur actuelle du surcroît net futur des revenus du travail, découlant de la réalisation d'une formation sup plémentaire, le coût encouru pour investir dans cette formation supplémentaire tout au long du cycle de vie, actualisé au moyen d'un taux de rendement social reflétant le coût d'opportunité que représente l'investissement dans une formation plus poussée.

4. Bien que le capital humain soit par définition le propre ou une caractéristique des individus, la somme ou la conjugaison des différentes quantités de capital humain individuel aboutit à un ensemble de compétences organisationnelles dont l'incidence est bien supérieure à celle de chaque composante.

5. Il y a des retomb ées lorsque l'amélioration des qualifications de certains contribue à accroître la productivité non seulement du personnel plus qualifié, mais aussi du personnel qui tire parti de cette productivité accrue.

6. La méthode de la «mécanique décisionnelle » a pour objet de repérer les apports distincts de l'école qui font une grande différence dans les résultats de l'école et des activités d'apprentissage.

7. Selon la méthode du « culturalisme en classe », les normes en vigueur en classe et la socialisation que la classe permet sont des aspects importants pour comprendre les résultats et l'efficacité de l'école.

8. Gundlach, Wossman et Gmelin (2000), se basant sur les résultats des élèves aux évaluations internationales en mathématiques et en sciences réalisées entre 1970 et 1994, affirment que la productivité de l'école, dans le meilleur des cas, n'a pas augmenté dans 9 pays de l'OCDE sur les 11 considérés, en dépit de l'accroissement des dépenses par élève.

9. Récemment, plusieurs tentatives ont été faites afin de déterminer des variables indicatives du capital culturel. Jusqu'à présent, elles ont été axées sur les activités de la famille à l'extérieur (assister à un concert symphonique, visiter une galerie de peinture, voyager, par exemple), la fréquence d'activités familiales au cours de la semaine (discussions en famille, dîner familial, débat sur des questions se posant à l'extérieur du foyer, par exemple), le type de documents à lire disponibles à la maison (quotidien, encyclopédies, recueils de poésies), ainsi que sur les langues comprises et parlées par la mère ou le père (voir Willms, 1999).

10. Des exemples de pratiques novatrices concernant les possibilités de formation proposées à proximité des lieux où les personnes vivent, travaillent et se déplacent sont passés en revue dans la publication « How Adults learn » (OCDE et Département de l'Éducation des États-Unis, 1998).

11. Ont participé à cette étude : I'Allemagne, la Corée, les États-Unis, le Japon, le Royaume-Uni et la Suède. 
12. Field et Spence (2000) dans leur étude sur la formation des adultes en Irlande du Nord constatent que si des liens sociaux denses d'ordre familial ou confessionnel sont associés à un niveau élevé de formation initiale, ces liens peuvent nuire à la participation des adultes à des activités formelles de formation. Cette situation tient peut-être en partie au fait que les liens denses au sein de la famille et de la collectivité se substituent à la participation à des activités formelles de formation continue. Du fait de l'absence de capital social pour relier les différentes communautés, les individus et les groupes locaux peuvent être faiblement incités à prendre part à des activités plus formelles ou structurées d'enseignement et de formation. En revanche, des liens intra-communautaires solides (analogues aux liens d'attachement examinés dans le chapitre 3) peuvent favoriser des niveaux plus élevés de participation des adultes à des ap prentissages informels car les adultes comptent sur les réseaux familiaux et communautaires pour effectuer les activités d'information et d'engagement social.

13. Angleterre et pays de Galles, États-Unis, Hongrie, Israël, Italie, Japon, Pays-Bas, Pologne, République fédérale d'Allemagne, République tchèque, Suède, Suisse et Taïwan.

14. L'enquête a été menée auprès de personnes âgées de 16 à 65 ans pour évaluer l'aptitude des adultes à utiliser des informations écrites et imp rimées. La compréhension de textes suivis couvre l'aptitude à lire et à comprendre un texte continu ; la comp réhension de textes schématiques concerne l'aptitude à interp réter des rapports, des documents et différents types de textes discontinus, tand is que la compréhension de textes au contenu quantitatif concerne la capacité d'interp réter des informations quantitatives, telles que des graphiques et des figures mathématiques, généralement contenues dans un texte suivi. La littératie a été mesurée non pas en termes de dichotomie personnes alphabétisées/analphabètes mais en fonction d'un continuum d'aptitudes mesurables.

15. Weiss (1995), entre autres, apporte un éclairage très différent sur la généralité théorique et la validité empirique des modèles de signalisation.

16. Bynner et al. (2001) ont également observé que si les adultes atteignaient les objectifs de littératie fixés à l'échelle nationale pour 2010, il pourrait en résulter une économie de 2.5 milliards de livres pour le contribuable au titre des compétences en calcul et de 0.4 milliard de livres au titre des compétences en lecture.

17. Temple (2001) précise : «Considérons par exemple l'affirmation selon laquelle $X$ points de pourcentage de croissance enregistrés dans un pays donné résultent d'une évolution de la qualité de la population active. Cela ne doit pas dire qu'en l'absence d'une telle évolution, le taux de croissance de la production aurait été plus faible précisément de $X$ points de pourcentage. En réalité, le niveau de formation peut avoir d'autres effets indirects sur la production à travers le taux d'activité, l'investissement et même la R-D ainsi que l'accroissement de la productivité totale des facteurs. L'analyse causale de la croissance ne rend pas compte de ces effets indirects et, en revanche, passe nécessairement sous silence l'importance globale que revêtent pour la croissance des variables telles que l'éducation ».

18. Par exemple, la relation entre le nombre d'années d'études et la production varie selon que l'on utilise les niveaux, le taux variation ou une présentation semi-logarithmique. Il est impossible de poser en hypothèse que le rendement de l'enseignement est indépendant du niveau initial.

19. Pritchett a aussi recours au taux d'accroissement de la productivité totale des facteurs dans une analyse causale de la croissance et arrive essentiellement à la même conclusion.m

20. Barro et Sala-i-Martin (1995), Barro et Lee (1997) constatent l'existence d'une relation inverse entre les niveaux initiaux de formation (secondaire et tertiaire) des femmes et la croissance.

21. D'autres études indiquent une forte corrélation entre le niveau d'instruction de la mère et l'état de santé de ses enfants.

22. La série Barro-Lee bien connue fournit des données chronologiques sur les niveaux de formation de la population de 25 ans et plus. Cette série de données a été établie essentiellement à partir des résultats du recensement recueillis par la Division des statistiques des Nations Unies, des estimations étant calculées pour les années manquantes.

23. Pour un échantillon plus large de pays, ils ont constaté que l'enseignement supérieur avait un impact important: ainsi, un allongement de la scolarité moyenne des garçons dans l'enseignement secondaire de 0.68 an majore la croissance annuelle de 1.1 point de pourcentage par an tand is qu'un al longement de 0.09 an en moyenne des études supérieures majore la croissance annuelle de 0.5 point de pourcentage. 
24. A l'aide de données recueillies pour 78 pays, McMahon examine les interactions existant entre différents avantages sociaux tels que la santé, la démocratisation, la réduction des inégalités dans la répartition des revenus et la pauvreté, les effets environnementaux et la délinquance. II constate que les effets de l'instruction sont dus pour plus de moitié à des effets ind irects ind uits, tels qu'une amélioration de la santé, qui se répercutent sur la croissance du revenu par habitant.

25. Il est donc possible d'utiliser les éléments d'information sur les préférences révélées des individus pour l'achat d'un niveau donné de santé financé par des ressources privées afin de calculer la valeur monétaire d'une élévation de l'instruction en additionnant la valeur correspondant à l'amélioration de divers résultats sociaux, dont une baisse de la délinquance, une meilleure santé, etc.

26. Voir Bynner et al. (2001), Bynner et Egerton (à paraître) et Schuller et al. (2001).

27. Car les travailleurs plus instruits ont tendance à travailler en moyenne de façon plus continue et les prélèvements fiscaux dont ils font l'objet sont proportionnellement supérieurs car du fait de leurs salaires plus élevés, ils sont imposés à des taux marginaux plus élevés. 



\section{Chapitre 3}

\section{LE RÔLE DU CAPITAL SOCIAL}

«Pratiquement toutes les transactions commerciales comportent un élément de confiance ; c'est vrai en tout cas de toutes les transactions menées sur une certaine durée. II est raisonnable de penser qu'une grande part du retard économique observé dans le monde s'explique par un manque de confiance mutuelle. » Kenneth J. Arrow, « Gifts and Exchanges », Philosophy and Public Affairs, 1, été 1972, p. 357.

\subsection{Introduction}

Une société fondée sur des relations de confiance et de coopération peut contribuer à réaliser le potentiel humain. Les ouvrages économiques témoignent d'une prise de conscience grandissante de l'importance que revêtent les réseaux sociaux et la confiance dans le soutien d'efforts collectifs. Ainsi, Temple (2001) observe, à propos du capital social, que «c'est une expression commode pour recouvrir tous les aspects des sociétés qui, bien que difficiles à mesurer et à intégrer dans des modèles structurés, sont généralement assimilés à des facteurs importants de réussite économique à long terme. Pour certains économistes (pas tous) l'idée intuitive que 'la société a son importance' est suffisamment forte pour qu'on la retienne malgré la quasi-absence de base théorique ».

Dans ce chapitre, on examine la définition, la mesure et l'impact du capital social. Cette expression étant relativement nouvelle et son acception n'étant pas universellement admise, on définira une méthode possible pour définir ce concept et mesurer sa contribution au bien-être. Bien que les éléments d'information dont on dispose soient pour l'instant préliminaires, ils donnent effectivement à penser que la notion de capital social est utile aux fins de l'action gouvernementale, et que des travaux plus poussés s'imposent pour affiner ce concept et mettre au point des techniques de mesure.

\subsection{Différentes conceptions du capital social}

Dans sa conception le « capital social » se différencie du capital humain et du capital physique à plusieurs égards ; en effet :

- il réside dans les relations et n'est pas la propriété exclusive de tel ou tel individu ;

- il constitue pour l'essentiel un bien public en ce sens qu'il est partagé par un groupe ; et

- il est le produit des investissements réalisés par la société en temps et en énergie, mais sous une forme moins directe que dans le cadre du capital humain ou physique.

Parallèlement aux compétences personnelles, les relations sociales jouent un rôle important dans la réussite.
Le capital social consiste en relations sociales et, en tant que capital, peut être conçu comme une ressource dans laquelle nous investissons pour obtenir un flux d'avantages. 
Le capital social dépend des actions menées par les individus qui le favorisent.

Cette notion a ses origines dans des recherches passées.
Le capital social est également le produit du patrimoine culturel et de normes de comportement héritées du passé. Le capital social présente donc des aspects d'ord re «social » puisqu'il réside plutôt dans des relations que dans des individus, mais aussi des aspects liés au «capital » puisqu'il est également une ressource pouvant générer un flux d'avantages pour la société au fil des ans. Cela dit, il peut également aboutir à un dysfonctionnement lorsqu'un groupe l'utilise contre d'autres.

De nombreux auteurs ont adopté une optique individualiste pour interp réter les incitations qu'ont les individus à utiliser le capital social et à investir dans son acquisition (Glaeser, 2001). Coleman a surtout vu dans le capital social un instrument permettant d'ob tenir certains résultats. D'autres, comme Hirschman, ont mis l'accent sur les efforts des individus pour œuvrer collectivement à la réalisation d'objectifs non monétaires tels que la justice, la beauté, l'amour, la cohésion et l'amitié. Cet aspect non instrumental de l'action collective représente « un investissement dans l'identité individuelle et de groupe » (Hirschman, 1984). Hirschman se focalise sur la valeur de "l'énergie sociale », telle que l'amitié, le partage d'idéaux et d'idées, qui transcende l'intérêt personnel rationnel et les transactions commerciales et favorise la coopération sociale. Quelle que soit la motivation qui pousse à coopérer et à faire confiance, l'investissement dans l'identité individuelle et de groupe peut aboutir à la création de réseaux sociaux denses qui permettent d'améliorer les résultats économiques et sociaux. Ainsi l'engagement civique, l'honnêteté et la confiance sociale peuvent-ils se renforcer mutuellement.

L'idée de capital social remonte aux travaux d'Alexis de Tocqueville1, d'Emile Durkheim² et de Max Weber3 (voir Woolcock, 1998, pour un historique complet de ce concept). La première mention connue de cette expression dans son acception contemporaine évoquait l'importance du capital social pour l'éducation et les communautés locales (Hanifan, 1916). Depuis cette époque, cette notion a été utilisée par Jacobs (1961)4 dans son analyse des quartiers urbains, par Loury (1987) dans une étude des marchés du travail, par Coleman (1988) qui a souligné sa complémentarité avec le capital humain, et par Putnam (1993) et Fukuyama (1995) qui I'ont appliquée au niveau de l'état-nation ou de la région (le premier insistant sur le rôle que joue l'engagement civique dans le développement de la démocratie et de la cohésion sociale). Bourdieu (1979) et Bourdieu et Passeron (1970) ont pour leur part utilisé la notion très proche de « capital culturel » (voir la section 2.4, chapitre 2).

Le capital social ne se prête pas à une seule et unique définition. Et I'on peut envisager ce concept sous au moins quatre principaux angles:

1. Les ouvrages anthropologiques sont à l'origine de l'idée selon laquelle les humains ont un instinct naturel d'association. Fukuyama (1999), par exemple, souligne le fondement biologique de l'ordre social, le capital humain ayant selon lui ses racines dans la nature humaine.

2. Les ouvrages de sociologie décrivent les normes sociales et les sources de la motivation humaine. Ils mettent en évidence les caractéristiques de l'organisation humaine, notamment la confiance, les normes de réciprocité et les réseaux d'engagement civique. 
3. Les ouvrages économiques reposent sur I'hypothèse que les personnes maximisent leur utilité personnelle, en décidant d'interagir avec d'autres, et ont recours aux ressources du capital social pour mener différents types d'activités de groupe (Glaeser, 2001). Dans cette optique, l'accent est mis sur les stratégies d'investissement des individus, qui ont le choix entre différentes utilisations possibles de leur temps.

4. L'un des courants dans les ouvrages de science politique souligne le rôle des institutions, des normes politiques et sociales dans la détermination du comportement humain. Dans des travaux récents sur le rôle joué par le capital social dans la réduction de la pauvreté et la promotion du développement durable, la Banque mondiale a insisté sur le rôle des institutions, des dispositions sociales, de la confiance et des réseaux 5 .

L'une des définitions a consisté à lier le concept de capital social à des aspects macro-institutionnels plus généraux que certains économistes appellent «capacités sociales » (Abramovitz et David, 1996; Omori, 2001 ; Hall et Jones, 1999 ; Temple et Johnson, 1998). Putnam (2000a), Woolcock (2001) et Knack (1999), en revanche, préfèrent une définition « dép ouillée » centrée sur les réseaux sociaux et les normes de réciprocité (ou de confiance dans le cas de Knack) qui leur sont directement liées. Certains observateurs (Woolcock, 2001) considèrent que la confiance découle du capital social (c'est-à-dire des réseaux et des normes s'y rapportant) tandis que pour d'autres, la confiance est une composante des valeurs et des normes communes qui constituent le capital social.

Dans ce rapport, le capital social correspond, par définition, à des réseaux ainsi qu'à des normes, valeurs et convictions communes. Les réseaux renvoient au comportement objectif $d$ 'acteurs qui mènent une activité en association. Les normes, valeurs et convictions communes renvoient aux dispositions et attitudes subjectives des individus et des groupes ainsi qu'aux sanctions et aux règles qui régissent les comportements, qui sont communes à un grand nombre6. Le contexte culturel dans lequel les attitudes, valeurs et connaissances communes sont transmises de génération en génération contribue puissamment à faire comprendre les choix que font les individus et les groupes à l'égard de la coopération. Des normes et des valeurs partagées permettent aux individus de communiquer entre eux et d'interp réter des expériences communes ainsi que les divergences à propos de certaines normes et valeurs. Des interprétations et des systèmes de valeurs à la fois différents et communs peuvent coexister sans nécessairement compromettre la coopération s'il y a un climat de tolérance. C'est pourquoi le dialogue et la compréhension mutuelle fondés sur la tolérance d'autres cultures ou croyances sont des aspects essentiels de la cohésion sociale et contribuent à étayer le capital social.

On peut considérer la confiance à la fois comme une source de capital social et un résultat de ce capital et comme une valeur de remplacement très proche de nombreuses normes, convictions et valeurs qui sous-tend ent la coopération sociale. II est possible d'établir une distinction en se demandant si les personnes font confiance aux autres et si les personnes sont dignes de confiance. La confiance peut être un bon indicateur de la loyauté (en tenant compte des décalages dans le temps), mais la loyauté décrit un compor-
Ce rapport propose sa propre définition du capital social.

La confiance favorise le capital social. 
Le capital social est en partie un bien public et en partie un bien privé.

\section{Le capital social comporte} trois types de liens.

Les liens d'attachement et d'accointance sont nécessaires pour éviter l'éclatement de la société. tement qui découle d'une multitude de facteurs dont les réseaux et les valeurs et normes communes. II y a lieu de distinguer trois types de confiance :

- la confiance entre personnes se connaissant (la famille, les collègues proches et les voisins) ;

- la confiance entre personnes « étrangères »; et

- la confiance dans les institutions publiques et privées.

Le capital social permet auxindividus, aux groupes et aux collectivités de résoudre plus facilement des problèmes communs. Les normes de réciprocité et les réseaux aident à assurer un comp ortement collectivement souhaitable. En leur absence, les individus tendent à ne pas coopérer parce qu'ils ne peuvent pas être sûrs que les autres vont faire de même. Le capital social peut être un sous-produit de diverses activités sociales qui ne sont pas nécessairement menées dans le but de le renforcer7. Comme le capital humain, le capital social a « des externalités positives » telles que de nombreuses personnes tirent parti de l'apport d'un individu ou d'un groupe au capital social. Il est par conséquent menacé de sous-investissement du fait que les acteurs qui contribuent à ce capital ne s'en approprient pas totalement les avantages. Comme l'observe Coleman : « Le résultat est qu'il va y avoir dans la société un déséquilibre d'investissement entre les organisations qui produisent des biens privés pour un marché et celles (souvent des associations) dont on ne saisit pas bien les avantages » (Coleman, 1990, p. 317). Cependant, l'accès aux informations et à l'influence que permettent les réseaux sociaux apporte aussi auxindividus des avantages privés et peut dans certains cas être utilisé par des individus ou des groupes pour en exclure d'autres et renforcer leur domination ou leurs privilèges.

Woolcock (1999) distingue dans le capital social trois formes de liens essentiels : les liens d'attachement entre membres d'une même famille et d'un même groupe ethnique ; les liens d'accointance avec les amis éloignés, les collègues et les associés ; enfin, les liens de contact entre les différentes couches sociales auxquelles accèdent différents groupes dans la hiérarchie des pouvoirs, des positions sociales et des richesses. Woolcock (2001) associe ce dernier type de liens à la capacité qu'ont les individus et les communautés de profiter des ressources, des idées et des informations émanant d'institutions situées au-delà de leur cercle immédiat.

Si les liens d'attachement donnent à des communautés ou à des groupes un sentiment d'identité et de finalité commune, sans les liens d'accointance qui transcendent diverses frontières sociales (religion, appartenance ethnique, situation socio-économique), les premiers peuvent conduire à la poursuite d'intérêts étroits et exclure les personnes qui n'en font pas partie. Des groupes relativement homogènes peuvent se caractériser par un haut degré de confiance et de coopération interne, mais un faible degré de confiance et de coopération vis-à-vis du reste de la société. Certaines formes de liens d'attachement exclusifs peuvent alors faire obstacle à la cohésion sociale et à l'épanouissement personnel : en pareil cas, les liens d'attachement sont forts mais les liens d'accointance sont faibles. Comme l'observent Powell et Smith-Doerr (1994) : «les liens d'attachement peuvent aussi être des liens aveuglants ». 
Si la confiance ne s'étend pas au-delà d'un groupe soudé - famille ou cercle restreint d'amis - les interactions sociales et la coopération avec le reste de la collectivité risquent d'être limitées (Knack, 1999 ; Portes et Land holt, 1996). Le souci exclusif des intérêts du groupe au détriment des intérêts collectifs plus vastes peut encourager une "recherche de rente » socialement destructrice (Olson, 1982 et Knack, 1999). Dans les entreprises, des liens étroits de confiance et d'obligation mutuelle peuvent, dans certaines circonstances, bloquer les informations provenant de l'extérieur et empêcher l'innovation (Kern, 1998 et Uzzi, 1997).

Bien que les liens ethniques puissent être « source d'avantages propices à l'adaptation » à l'arrivée des groupes d'immigrants dans un pays, des liens ethniques exclusifs peuvent empêcher les individus d'élargir leurs contacts à un réseau plus vaste. Cela montre bien l'importance des liens d'accointance lorsqu'il existe une grande diversité de groupes, ethniques ou autres.

Certaines formes particulières de liens d'attachement peuvent dans certaines circonstances empêcher la cohésion sociale. A cet égard, le capital social ne diffère pas des autres formes de capital, qui peuvent être utilisées à diverses fins - pas toutes nécessairement souhaitables pour l'ensemble de la collectivité. Certains groupes dont les liens sont très forts tels que les cartels de la drogue, les filières d'immigration clandestine, les groupes maffieux et terroristes, peuvent se caractériser par des niveaux élevés de confiance interne et de récip rocité8. Ces mêmes groupes peuvent aussi comprendre des individus dotés d'un haut niveau de capital humain, qui utilisent des capitaux financiers, entre autres, à des fins socialement destructrices et indésirables. Certains types de liens d'accointance trop exclusifs à l'échelon national ou régional peuvent avoir des conséquences socialement destructrices 9 . Ces exemples ne diminuent pas le potentiel qu'a par ailleurs le capital humain ou social de générer des avantages pour tous les membres de la société ou la plupart d'entre eux. Les avantages qu'apportent la plupart des types de liens sociaux ont en général beaucoup plus de poids que leurs inconvénients.

\subsection{Comment mesurons-nous le capital social ?}

Il est difficile de mesurer le capital social. En règle générale, la plupart des mesures dont on dispose portent sur la confiance et les niveaux d'engagement ou d'interaction dans des activités sociales ou des activités de groupe. Dans son analyse des différences de capital social à travers les États-Unis, Putnam (2000a) a eu recours à de vastes séries de données transversales et longitudinales. Ses mesures du capital social sont en général fondées sur un indice composite dont les éléments sont les suivants : i) le degré de participation à la vie organisationnelle et à la collectivité ; ii) l'engagement dans la vie publique (par exemple, la participation au vote) ; iii) le bénévolat; iv) la sociabilité informelle (rencontres avec des amis) ; et v) les niveaux subjectifs de confiance entre personnes.

En principe, il faudrait que les mesures du capital social i) couvrent le plus grand nombre possible d'aspects essentiels (réseaux, valeurs et normes) et ii) respectent un équilibre entre les éléments subjectifs liés à l'attitude (niveaux de confiance déclarés, par exemple) d'une part et les aspects comportementaux (par exemple, l'appartenance à des associa-
Les groupes soudés peuvent exclure les gens qui $n$ 'en font pas partie.

On reconnaît les inconvénients de certaines formes de capital social.

La mesure du capital social se trouve encore à un stade embryonnaire... 
... mais des instruments pilotes de mesure sont mis au point.

Les dysfonctionnements sociaux constituent une mesure négative du capital social...

... mais la World Values Study fournit une mesure directe de la confiance. tions et l'étendue des liens sociaux) d'autre part. Il faudrait utiliser ces mesures en tenant compte du contexte culturel dans lequel sont mesurés les comportements ou les attitudes en rapport avec le capital social (en effet, dans les enquêtes, les questions posées au sujet de la confiance peuvent s'entendre très différemment selon les pays).

Toutefois, les difficultés rencontrées pour mesurer le capital social doivent être reconnues. Le désir d'effectuer une mesure peut amener à confondre les sources, les fonctions et les résultats. Nombre des aspects qui ont trait au capital social sont tacites et relationnels et ne se prêtent pas aisément à la mesure ou à la codification. Les attitudes des individus (la confiance par exemple) ou leurs comportements (notamment, leur ad hésion à des organisations, leur participation aux votes) sont des mesures indicatives du capital social, mais il ne faut pas toutefois confondre la mesure indicative avec le concept lui-même. Les efforts faits pour refléter les aspects essentiels des interactions et des relations entre les gens se heurtent au manque de sources de données ap propriées, qui lui-même est dû au fait que les questionnaires d'enquête ne comprennent pas un éventail suffisamment complet de questions et que les enquêtes ne sont pas véritablement conçues pour mesurer le capital social. C'est pourquoi il est difficile de recueillir des données sur ce sujet à l'échelon international.

La Banque mondiale a mis au point des mesures du capital social «par le bas », à partir d'enquêtes ou de modules d'enquête spécialement conçus. C'est ainsi que Narayan et Pritchett (1998) ont construit une mesure du capital social en Tanzanie rurale, à partir des données de l'enquête sur le capital social et la pauvreté (SCPS) dans ce pays. Cette enquête à grande échelle a consisté à poser aux individus des questions sur l'importance et les caractéristiques de leurs activités associatives, et sur la confiance qu'ils accordaient à diverses institutions et personnalités. Un certain nombre de nouvelles enquêtes ou de nouveaux modules d'enquête sont par ailleurs en cours d'élaboration au Royaume-Uni et en Australie (voir Cox et Macdonald, 2000 et Schuller et al., 2001).

Un moyen d'évaluer les variations du capital social consiste à mesurer les dysfonctionnements sociaux ou l'absence de coopération sociale. Fukuyama mesure ces variations à partir de l'évolution de la criminalité, de l'éclatement des familles et de la confiance. A l'augmentation des taux de criminalité on peut apparenter celle de la « déviance sociale » (développement des incivilités et du manque d'esp rit civique, etc.). Dans cette méthode, les variations sur une longue période des mœurs et des valeurs sont considérées comme des indicateurs des variations du capital social. Cependant, il faut être prudent lorsqu'on utilise des indicateurs des dysfonctionnements sociaux, car on n'en connaît pas toutes les causes et le capital social n'est que l'un des facteurs qui peut y contribuer. De plus, on risque dans ce type d'approche de confondre conséquences et origines.

A diverses reprises (en 1981, 1991 et 1996), on a inclus dans la World Values Study 10 une question sur la disposition des personnes interrogées à faire confiance à autrui : " D'une manière générale, diriez-vous qu'on peut faire confiance à la plupart des gens, ou non ? ». Les chiffres montrent que les niveaux de confiance déclarés diffèrent beaucoup selon les pays Membres de I'OCDE (voir le tableau 3.1), que les écarts restent relativement stables dans le tempsil et que les pays voisins tend ent à afficher des 
valeurs proches (ce qui témoigne de la force des antécédents culturels régionaux).

Tableau 3.1. Mesure de la confiance (World Values Study), 1995-96

Pourcentage de personnes déclarant qu'on peut faire confiance à la plupart des gens

\begin{tabular}{|c|c|c|c|}
\hline \multicolumn{4}{|c|}{ Membres de I'OCDE } \\
\hline Norvège & 65.3 & Italie * & 35.3 \\
\hline Suède & 59.7 & Belgique * & 33.2 \\
\hline Danemark* & 57.7 & Autriche * & 31.8 \\
\hline Pays-Bas* & 55.8 & Royaume-Uni & 31.0 \\
\hline Canada* & 52.4 & Corée & 30.3 \\
\hline Finlande & 47.6 & République tchèque * & 30.3 \\
\hline Irlande * & 47.4 & Espagne & 29.8 \\
\hline Japon & 46.0 & Mexique & 28.1 \\
\hline Islande * & 43.6 & Hongrie * & 24.6 \\
\hline Allemagne & 41.8 & France $*$ & 22.8 \\
\hline Suisse & 41.0 & Portugal * & 21.4 \\
\hline Australie & 39.9 & Turquie & 6.5 \\
\hline États-Unis & 35.6 & & \\
\hline \multicolumn{4}{|c|}{ Sélection de pays non-membres de l'OCDE } \\
\hline Inde & 37.9 & Afrique du Sud & 18.2 \\
\hline Chili & 21.9 & Argentine & 17.5 \\
\hline Nigeria & 19.2 & Brésil & 2.8 \\
\hline
\end{tabular}

L'une des difficultés à propos de ce type de question est que l'on ne sait pas au juste si les personnes interrogées interp rètent de la même façon les termes «confiance » ou «la plupart des gens», quels que soient leur pays, leur culture et l'époque à laquelle la question leur est posée. Par ailleurs, il faut faire une distinction entre la disposition des individus à faire confiance d'une part à des personnes qu'ils connaissent et d'autre part à « autrui » en général (renvoie à la distinction établie entre la confiance à l'égard de personnes connues et à l'égard de personnes étrangères mentionnée plus haut). L'environnement, le contexte et la situation jouent un rôle déterminant. Dans des pays comme I'Espagne, la France et I'Italie, où les niveaux de confiance à l'égard d'étrangers paraissent être faibles comparés aux autres pays de l'OCDE, les individus déclarent des niveaux élevés de confiance vis-à-vis de leur cercle familial immédiat (selon la World Values Study - voir Galland, 1999). Bien qu'il y ait une corrélation entre la confiance et l'engagement civique, Galland constate des différences importantes entre les groupes sociaux dans la façon dont les individus exercent leur choix de réseaux et de relations. Un haut niveau de confiance dans un domaine peut coexister avec un degré restreint d'engagement ou de confiance dans un autre. Galland conclut que ni les mesures générales de confiance ni l'engagement civique ne donne une indication fiable de la qualité des relations sociales ou de leur interaction au niveau macro. II s'ensuit que les résultats d'une enquête comme la World Values Study doivent être considérés avec prudence.
La mesure de la confiance doit être interprétée avec prudence. 
La confiance et l'engagement civique seront probablement imbriqués.
Knack et Keefer (1997) rapportent une expérience qui a consisté à « perdre », dans un certain nombre de villes, un grand nomb re de porte-monnaie contenant 50 dollars. Le pourcentage de porte-monnaie «perdus » qui ont été rendus à leurs propriétaires dans chaque pays présentait une corrélation de 0.67 avec la mesure de la confiance, ce qui tend à indiquer que les gens sont effectivement plus dignes de confiance dans les pays où l'indice de confiance est élevé12. Knack et Keefer ont par ailleurs construit un deuxième indice, destiné à mesurer la force des normes de loyauté et de coopération civique. Cet indice représente la moyenne des réponses à cinq questions concernant l'attitude adoptée face à des comportements tels que la fraude aux prestations ou la fraude dans les transports en commun. Cet indice présentait relativement peu de variations entre les différents pays de I'OCDE. Putnam (2000a) montre que dans les États des États-Unis, une forte corrélation existe entre la confiance et d'autres mesures du capital social lié à l'engagement civique et aux liens sociaux. On peut donc penser que la confiance est peut être un indicateur acceptable du capital social, faute de disposer d'une série d'indicateurs plus large et plus complète.

\subsection{Les sources du capital social}

Le capital social se constitue au « niveau » des familles, des communautés, des entreprises et des unités administratives et autres institutions nationales ou infranationales. D'une façon générale, l'idée de capital social est associée aux relations qui existent dans la société civile. Or, les relations de confiance et les réseaux font aussi intervenir les institutions et organisations publiques. Le capital social a ses racines dans les normes et institutions, parmi lesquelles figurent les entités publiques et légales. L'analyse peut aussi porter sur les différents groupes qui composent la société civile et qui se définissent par le sexe, la profession, la langue ou l'ethnie. On peut retenir, comme sources et dimensions du développement du capital social : i) la famille ; ii) l'école ; iii) la communauté locale ; iv) l'entreprise ; v) la société civile ; vi) le secteur public ; vii) la complémentarité homme-femme ; et viii) I'ap partenance ethnique. On a accordé une grande attention au rôle des associations bénévoles et civiques, mais le rôle clé de la famille, de l'école et de l'entreprise a peut-être été relativement négligé dans les récents débats et analyses au sujet du capital social.

La famille est le socle du capital social.
La famille crée des normes et des liens sociaux et constitue un réseau social qui est source d'avantages pour ses membres - surtout pour les familles élargies dans les « sociétés familiales » (voir Coleman, 1990 et Bourdieu, 1985, 1979). Les relations familiales fondées sur la réciprocité et l'échange et sur l'aptitude à répond re aux besoins affectifs et matériels des enfants peuvent aussi favoriser le développement de la confiance et de la coopération en dehors du cercle familial immédiat. Le soutien matériel et affectif, que les membres de la famille s'apportent mutuellement peut en effet disposer ces derniers à reproduire le même schéma à l'extérieur de la famille et faire qu'ils s'attendent à bénéficier de ce soutien aussi à l'extérieur. La famille est aussi une source première d'apprentissage, et peut stimuler la réussite scolaire. La famille peut ajouter une influence positive indirecte aux effets potentiellement importants de l'éducation sur le développement du capital social (voir plus loin). Pour mesurer le capital social de la famille, Coleman utilise comme indicateurs : i) la présence physique d'adultes ou de parents dans le foyer, et ii) la qualité et 
I'intensité de l'attention accordée aux enfants par les adultes ou les parents. On peut également mesurer ces deux dernières variables à l'aide du ratio adultes/enfants. D'une manière générale, ap rès neutralisation des autres facteurs, plus la fratrie est grande et plus le nombre d'adultes présents à la maison est faible, moins les apprenants disposent de capital social. Toutefois, des liens familiaux forts (liens d'attachement) peuvent dans certains cas constituer un frein à de plus grands liens d'accointance.

Les relations sociales, qui entrent dans la composition du capital social, sont plus fréquemment rompues lorsque les familles déménagent (Glaeser, 2001). Le capital social est généralement moins développé dans les familles monoparentales, toutes choses égales d'ailleurs. La qualité de l'attention accordée à chaque enfant est d'autant meilleure que les adultes sont nombreux au sein du foyer, que le nombre d'enfants est réduit et le lieu de résidence de la famille stable (MCLanahan et Sand efur, 1994, et Hao, 1994). De leur tour d'horizon des recherches menées aux États-Unis, McLanahan et Sandefur ont conclu que le divorce - qui s'accompagne souvent d'une baisse de revenus, d'une moindre présence des parents auprès des enfants et d'un accès plus limité aux services collectifs - a généralement pour effet de diminuer le bien-être des enfants et de faire baisser leurs résultats scolaires. Un certain nombre d'études (Biblraz, Raftery et Bucur, 1997 et Simons, 1996) montrent qu'il existe une corrélation entre le fait pour les enfants de vivre dans une famille monoparentale ou reconstituée, et un certain nombre de handicaps sociaux comme, par exemple, des taux plus faibles d'achèvement des études, des taux plus élevés de criminalité ou de toxicomanie, et une plus forte proportion de grossesses précoces. Painter et Levine (1999) confirment cet argument : à partir de données longitudinales recueillies aux États-Unis, ils ont constaté que, entre la structure familiale et le niveau de bien-être des enfants, mesuré de diverses façons, il existe un lien de cause à effet plus déterminant que les caractéristiques inhérentes aux familles à risque. Toutefois, ces effets peuvent être en partie ou totalement compensés par d'autres facteurs 13 .

Les établissements scolaires peuvent promouvoir les valeurs propices à la coopération sociale tout en étant des « lieux de rencontre » où divers réseaux sociaux peuvent se côtoyer. D'une façon plus générale, les établissements d'enseignement supérieur, les organismes de formation pour adultes et les organisations professionnelles peuvent favoriser la création de réseaux regroupant différents secteurs - celui de la formation, l'entrep rise et les mouvements associatifs. Dans la mesure où les méthodes d'enseignement et l'organisation de l'ap prentissage encouragent la mise en commun des connaissances, le travail en équipe ainsi que l'ouverture aux idées nouvelles et à la diversité culturelle, un plus grand nombre d'établissements scolaires peuvent étayer le capital social qui relie différents groupes de la société.

Les communautés et le voisinage jouent aussi un rôle dans la formation du capital social. Les interactions sociales entre voisins, amis et groupes génèrent du capital social et l'aptitude à travailler ensemble pour le bien commun. Coleman (1990) établit un lien entre la famille et la communauté locale lorsqu'il analyse l'« enfermement » par lequel une communauté peut peser sur ses membres en les menaçant de sanctions lorsqu'ils ne respectent pas certaines normes. Loury14 (1987) considère le capital social comme l'un des « biens publics locaux» que produit la collectivité. Ces biens publics
Les établissements scolaires et les organismes de formation peuvent également développer le capital social...

... tout comme le peuvent les communautés locales... 
... et les entreprises.

Les associations et les organisations bénévoles jouent aussi un rôle. locaux peuvent être d'ordre très général ; ce sont par exemple l'éducation, l'influence exercée par les pairs, les contacts, les réseaux d'amis.

Parmi les ouvrages, de plus en plus nombreux, consacrés à la «nouvelle économie », certains (celui de Drucker, par exemple) soulignent l'importance des réseaux, de la confiance, des partenariats et des collaborations. L'idée, en effet, est que l'innovation s'appuie de plus en plus sur la coopération, l'apprentissage rapide et les réseaux. Et les réseaux innovants intra ou intersectoriels qui lient fournisseurs, clients et chercheurs suscitent un fort potentiel de croissance. Le regroupement d'activités, avec tous les avantages de la collaboration qu'offrent les réseaux, a peut-être une corrélation avec l'avantage compétitif national (Porter, 1990). L'aptitude à « se mettre en réseau » ou à communiquer en dehors des circuits formels prend alors de plus en plus d'importance. Les organisations tayloristes, fondées sur des règles formelles et des structures de pouvoir hiérarchiques, font moins appel aux réseaux et à la confiance que les organisations post-fordistes, où l'autorité et les responsabilités de gestion sont plus internalisées et où la confiance et le partage de l'information sont plus déterminants, mais qui par conséquent exigent de plus hauts niveaux de compétence. Les réseaux informels qui favorisent la transmission des savoirs tacites sont importants. Les organisations qui « apprennent » à socialiser le savoir et les compétences grâce à des formes plus efficaces d'interaction, de réseaux et de normes de confiance et de coopération sont des sources importantes de capital social.

La société civile est une autre source de capital social. Elle se compose des « groupes et organisations, formels et informels, qui interviennent indépendamment de l'État et du marché pour promouvoir divers intérêts dans la société $\gg 15$. Les relations entre la société civile, l'État et le marché comportent de fortes synergies. L'ampleur et la densité des relations entre groupes peuvent déterminer l'efficacité avec laquelle ces synergies vont favoriser le bien-être et la croissance économique. Dans son analyse du capital social dans différentes régions d'Italie, Putnam (1993) explique les différences de performance économique et politique entre les régions par le capital social constitué au fil du temps à travers divers types d'associations et de traditions civiques. Putnam souligne l'importance de divers types d'associations entre les gens (clubs sportifs, coopératives, mutuelles, associations culturelles, syndicats, etc.). Ces groupes favorisent une accumulation de liens d'attachement et de liens d'accointance, qui entraîne des effets positifs, parmi lesquels une meilleure efficacité des institutions politiques. Ainsi, pour Putnam, les interactions entre les membres de ces groupes et organisations créent des réseaux horizontaux d'engagement civique qui aident leurs participants à agir collectivement de telle façon que la productivité et le bien-être de la communauté s'en trouvent améliorés. En bref, les associations et les réseaux peuvent, comme l'école, développer la confiance et l'engagement civique, ce qui pourra entraîner des retombées positives dans d'autres secteurs de la société, notamment l'État et le marché.

Une gestion des affaires publiques inspirée par une volonté de bien-être public, de responsabilité et de transparence, est l'une des assises de la confiance et de l'insertion sociale, et peut donc renforcer indirectement le capital social. Les conditions politiques, institutionnelles et juridiques qui prévalent dans un pays peuvent servir à étayer les réseaux et les normes de coopération sociale. Ces deux catégories de facteurs peuvent se compléter et se renforcer mutuellement pour le bien-être général : non seu- 
lement le capital social permet une meilleure gestion des affaires publiques et une plus grande efficacité des institutions politiques, mais ces dernières peuvent compléter les réseaux locaux (sans les remplacer) et renforcer la confiance.

Les niveaux de confiance et d'engagement social et les types d'engagement (formel, informel) peuvent être très différents entre les hommes et les femmes, qui n'ont pas les mêmes réseaux sociaux ni les mêmes niveaux d'accès à l'information. Dans certains cas, les réseaux des hommes tend ent à être plus formels du fait qu'ils occupent plus souvent un emploi, tandis que les réseaux des femmes tend ent à être plus informels et plus centrés sur la famille (Moore, 1990). La capacité de confiance de l'enfant dépend surtout de la relation qu'il a avec sa mère, car c'est très souvent la mère qui s'occupe essentiellement des enfants - même si la qualité de la relation avec le père a son importance (Amato, 1998, p. 247). La participation active des femmes à la vie civique, professionnelle et politique peut aussi contribuer au capital social, ce qui veut dire que les discriminations fondées sur le sexe peuvent nuire à l'édification de certaines formes de capital social16.

Les liens ethniques montrent également comment des acteurs qui ont des valeurs et une culture commune peuvent s'associer au profit de tous. Portes et Sensenbrenner (1993) notent que des éléments tels que la réciprocité, ainsi que l'obligation de solidarité et de confiance (c'est-à-dire sociales) sont des caractéristiques essentielles des relations entre l'appartenance ethnique et le capital social. Pour les groupes d'immigrés, cette appartenance est une « source d'avantages propices à l'adaptation » au moment de leur arrivée dans leur nouveau pays car ils comptent sur le capital social pour obtenir des conseils et un soutien dans de multiples domaines. Ainsi, les groupes ethniques sont des sources de capital financier et humain pour ceux de leurs membres qui veulent créer une entreprise et qui, grâce aux solides liens existant au sein du groupe, peuvent obtenir des prêts et des subventions ainsi qu'une main-d'œuvre bon marché (Geertz, 1962). Ils peuvent souvent fournir de précieux renseignements sur le marché du travail local. Ils offrent aussi un filet de sécurité sociale en répond ant aux besoins matériels et financiers durant les périodes difficiles et en s'occupant des enfants et des personnes âgées (Abrams et Bulmer, 1986). Bien sûr, l'appartenance ethnique peut aussi parallèlement servir à créer entre certaines personnes des liens dont d'autres sont exclues.

\subsection{La confiance et la participation à la vie civique évoluent-elles au fil du temps?}

Les variations de niveaux du capital social dénotent des variations à plus long terme des normes, valeurs et schémas d'interaction sociale. Putnam (2000a) a constaté aux États-Unis une diminution de l'engagement social et civique. La baisse observée auxÉtats-Unis ne se retrouve pas tout à fait dans d'autres pays. Au Royaume-Uni et en Suède, l'appartenance à divers types d'organisation civile a progressé, aussi bien en chiffres absolus qu'en proportion de la population totale. Dans certains pays (notamment en Australie), la nature de l'engagement civique a évolué, devenant plus individualiste et temporaire. On constate dans certains pays des différences de niveau de confiance et d'engagement civique selon le groupe d'âge.
Le sexe est un déterminant essentiel de l'accès au capital social...

... tout comme l'est l'origine ethnique.

On constate un

désengagement civique dans certains pays, mais pas dans d'autres. 
On ne constate guère de baisse de l'engagement social ailleurs qu'en Australie et aux États-Unis.

Certaines organisations civiques traditionnelles semblent toutefois en perte de vitesse...

... et de nouvelles formes d'engagement sont apparues qui sont plus axées sur des thèmes particuliers.
Si des formes traditionnelles locales de liens sociaux (familles, quartiers et organisations de masse traditionnelles) semblent sur le déclin dans de nombreux pays de l'OCDE, on voit apparaître dans le même temps d'autres formes de liens sociaux, souvent distants, temporaires et davantage dictés par l'intérêt personnel. Toute la question est de savoir si ces nouvelles formes de liens sociaux sont suffisantes pour remplacer les anciennes. Les changements que l'on constate dans le domaine de l'engagement civique, de la participation au vote, de l'appartenance à divers types d'organisations, des niveaux de confiance et de la socialisation informelle sont autant d'indications importantes des tendances qui se dessinent dans les pays de I'OCDE. Nous examinerons ici ce qui se passe dans quelques pays de I'OCDE (Allemagne, Australie, France, Japon, Pays-Bas, Royaume-Uni, Suède et États-Unis). L'annexe E comprend plus de précisions.

Les informations dont on dispose mettent en lumière :

- une participation aux organisations civiques stable ou en augmentation (sauf en Australie et aux États-Unis) ;

- des niveaux de bénévolat stables ou croissants dans la plupart des pays ;

- des signes contrastés de baisse de l'engagement des jeunes dans les organisations et le bénévolat ;

- des signes contrastés d'une baisse des niveaux de sociabilité informelle (mais la qualité et l'exhaustivité des données laissent à désirer) ;

- des signes contrastés de baisse des niveaux de l'intérêt politique et du militantisme.

- une baisse de la participation à certaines formes plus traditionnelles d'organisations civiques telles que les organisations syndicales, confessionnelles et féminines ;

- certains signes de baisse des niveaux de confiance interpersonnelle et de confiance dans les institutions politiques (surtout chez les jeunes au Royaume-Uni et aux États-Unis).

- le développement de nouveaux mouvements pour la défense de causes diverses (I'environnement, entre autres) durant ces dernières décennies ;

- l'augmentation du nombre d'adhérents à des organisations culturelles et sportives dans la plupart des pays ;

- des modes de participation à la vie politique donnant moins de place aux contacts directs et plus aux entretiens avec des professionnels, les médias jouant un rôle prépondérant ;

- une certaine évolution vers des formes plus informelles de rapports sociaux. Ceux-ci tendent à être plus individualistes et temporaires, mais pas nécessairement plus matérialistes ou égoïstes ; enfin 
- de nouvelles formes de participation civique plus étroites, plus individualistes et moins orientées vers des intérêts ou des objectifs collectifs ou de groupe. On assiste peut-être à une certaine forme de privatisation du capital social.

A partir des données internationales de la World Values Study, Inglehart (1997) a également noté une diminution des niveaux de confiance dans différents types d'institutions, y compris les hiérarchies politiques et religieuses. Les niveaux déclarés de confiance dans l'État, la fonction publique, la police, l'église, le système d'éducation et les médias auraient baissé dans la plupart des pays entre 1981 et 1990. En revanche, on constate durant la même période une relative stabilité, voire une certaine augmentation de la confiance entre personnes. Cette évolution est peut-être liée à l'élévation des niveaux d'instruction et à un déplacement des valeurs vers plus d'autonomie personnelle et moins de soumission à l'autorité.

\subsection{Quelle est l'origine de l'évolution des niveaux de confiance et de par- ticipation à la vie civique?}

La baisse du capital social aux États-Unis et en Australie est-elle un phénomène exceptionnel, ou annonce-t-elle une évolution analogue dans les autres pays? Les données dont on dispose montrent que les effets intergénérationnels sont particulièrement importants aux États-Unis. Les générations récentes (en particulier celles nées depuis les années 1950) ont en général moins tend ance à adhérer à des organisations ou à des associations, à voter ou à s'engager socialement, et à faire confiance aux autres. Putnam (2000a) conclut que l'effet des différences intergénérationnelles est particulièrement important à long terme et l'emporte sur l'effet de la classe sociale, du groupe ethnique ou du lieu géographique. II arrive à cette conclusion en recourant à diverses sources de données qui permettent de faire la distinction entre les variations qui interviennent au cours de l'existence (l'engagement civique est maximum à l'âge mûr), les différences entre cohortes à des moments précis (les générations baby-boom comparées aux générations plus anciennes) et les variations associées à des périodes spécifiques (qui reflètent l'impact de changements soudains sur l'ensemble des cohortes).

II n'est pas facile de savoir pourquoi ces effets intergénérationnels sont si importants. Putnam signale que la Deuxième Guerre mondiale a donné lieu à un formidable esprit civique national, dont on a ressenti les effets chez les générations nées entre 1910 et 1940. La réapparition du capital social au début du 20e siècle aux États-Unis (après une période de déclin vers la fin du 19e siècle, qui n'est pas sans ressembler aux tendances récentes) a été liée à divers mouvements de renouveau civique qui s'y sont produits au tournant du siècle. Dans son analyse, Putnam se demande si l'évolution analogue observée en Australie est également attribuable aux mêmes effets intergénérationnels et pourquoi on ne les constate pas aussi dans d'autres pays.

La plupart des pays de I'OCDE connaissent actuellement d'importants changements dans leurs systèmes de valeurs, leurs normes et leurs schémas de comportements sociaux. Pour certains observateurs (par exemple Inglehart, 1997), ils font partie d'une évolution naturelle des sociétés moder-
La confiance entre individus augmente, mais la confiance dans les institutions diminue.

L'évolution entre générations est particulièrement importante aux États-Unis.

Des changements plus vastes se produisent dans les systèmes de valeur... 
... impliquant souvent un attachement plus faible à des causes communes.

De nouvelles formes d'engagement pourraient apparaître.

Le temps passé devant la télévision est lié à une baisse de l'engagement civique... nes vers davantage d'autonomie individuelle, moins de contrôle hiérarchique, une dilution des identités collectives et culturelles et, dans certains cas, des objectifs post-matérialistes ou « post-modernes » et individualistes. Inglehart (1997) et Yankelovich (1981) laissent entrevoir en outre que l'accroissement des richesses matérielles est une cause majeure de perturbation sociale et de dysfonctionnement du développement économique dans ses phases initiale et intermédiaire, tandis qu'à un stade ultérieur de ce développement, une sécurité économique relative pourrait entraîner un retour à des valeurs plus traditionnelles. La conséquence de cette évolution est que les liens sociaux pourraient changer plutôt que diminuer.

La progression de nouvelles valeurs mettant davantage l'accent sur l'autonomie et une perte de respect peuvent avoir quelques effets ind ésirables tel qu'un recul du sens du devoir et de l'entreprise commune. Alors qu'à une époque, les individus pouvaient compter sur le soutien de la famille élargie, de l'Église et d'autres organes sociaux, aujourd 'hui, ces soutiens traditionnels se sont affaiblis. Une des conséquences est que le passage à l'âge adulte risque d'être plus délicat pour les jeunes qui sont à la recherche d'une identité, de croyances et d'une place dans la société. Pour certains commentateurs (comme Fukuyama, 1999), la dégradation des valeurs communes, des identités communes et des objectifs sociaux à poursuivre, affaiblit la confiance et la volonté d'un engagement civique de qualité au service de l'intérêt général.

Selon Rothstein (1998), il se peut que les gens partagent de moins en moins les mêmes valeurs et les mêmes modes de vie, mais soient plus concernés et plus engagés socialement. A partir de données d'enquêtes suéd oises, il conclut que les individus sont plus disposés à ap porter une aide à d'autres individus, mais aussi à admettre que ceux-ci aient d'autres valeurs et veuillent s'engager pour des causes différentes. On peut se demander si ce constat pourrait s'appliquer à d'autres pays, mais il illustre l'un des grands défis que pose cette transformation des valeurs et des normes. Souvent, il ne sera guère logique de tenter de recréer les traditions passées et il faudra plutôt s'efforcer de susciter dans la société un renouveau d'engagement civique et public fondé sur les aspirations et les attitudes nouvelles.

Putnam (2000a) observe un lien étroit entre le temps de plus en plus long que passent les Américains devant la télévision (en même temps qu'ils sont moins sélectifs dans leurs choix de programmes) et le déclin de l'engagement social, ind épendamment d'autres facteurs comme la race, le statut social et l'âge. La conjugaison des effets intergénérationnels et de l'allongement du temps passé devant la télévision semble expliquer l'essentiel du déclin du capital social aux États-Unis depuis le milieu des années 1960. Aux PaysBas, de Hart et Dekker (1999) observent en effet un lien entre le temps passé devant la télévision et le désengagement civique (et expliquent ainsi non pas le déclin de l'ensemble du capital social, mais plutôt le fait que certains groupes sont plus engagés que d'autres). Ils constatent que les personnes qui regard ent beaucoup la télévision consacrent relativement peu de temps à rendre visite à des amis et connaissances, à faire du bénévolat, à recevoir, à parler aux autres membres de la famille, à écrire ou à téléphoner17. Des conclusions de ce genre amènent à se demander pourquoi, étant donné que le temps passé à regard er la télévision augmente globalement, la baisse du capital social s'observe de façon très nette uniquement aux États-Unis et peut-être en Australie. 
Comme l'affirme Putnam (2000a, p. 170), étant donné le caractère récent de l'Internet, il est difficile de dire qu'il est l'une des principales causes de l'évolution ou de la baisse du capital social - surtout aux ÉtatsUnis où ce recul a commencé 25 ans plus tôt. Les effets de I'Internet à I'avenir pourraient être considérables. II importe de noter que si la communication par voie informatique est jugée favorable à la mise en commun de l'information, au recueil d'opinions et au débat entre des personnes physiquement éloignées, on a souvent considéré qu'il était plus difficile de construire des relations de confiance dans un environnement virtuel. En réalité, la communication par l'Internet paraît plutôt compléter les interactions sociales directes que de s'y substituer.

Putnam affirme qu'auxÉtats-Unis l'augmentation du temps de travail, les familles à double revenu et l'étalement des villes sont des facteurs qui interviennent dans le déclin du capital social, mais de façon moins significative que les différences intergénérationnelles et le temps passé devant la télévision. Le fait qu'on réside de moins en moins dans des zones rurales multifonctionnelles et de plus en plus dans des zones urbaines et suburbaines uniquement vouées à l'habitat semble avoir accéléré le déclin de l'engagement civique. En revanche, Putnam ne constate pas d'impact majeur de l'emploi des femmes sur leur participation à la vie collective. En neutralisant l'effet du niveau d'instruction, de la situation matrimoniale et de l'année de naissance, Putnam constate que les femmes sont plus engagées socialement que les hommes dans toutes les catégories d'emploi (temps complet, temps partiel et sans emploi). Celles qui occupent des emplois à temps partiel (surtout si elles ont trouvé l'équilibre qu'elles souhaitent entre le travail et la famille) tendent à être plus engagées socialement que les autres 18. La conclusion qui s'impose est donc que la baisse de la participation à la collectivité aux États-Unis existe pour toutes les catégories d'emploi, y compris les femmes qui n'occupent pas un emploi rémunéré, ce qui révèle que l'évolution de la situation des femmes vis-à-vis de l'emploi n'est pas un facteur majeur de la baisse de l'engagement civique. AuxÉtatsUnis, cependant, le manque de temps lié à l'augmentation du temps de travail a pu jouer un certain rôle aussi bien chez les hommes que chez les femmes.

Il a été dit que le système de protection sociale pouvait être une cause possible du déclin du capital social. L'argument avancé est que s'il y a une prise en charge importante des obligations sociales par le secteur public, les liens associatifs, familiaux et interpersonnels tendent à s'affaiblir et les particuliers sont moins incités à assurer des services bénévoles (Norton, 1998). Autre possibilité, l'État-providence, au lieu d'annihiler I'initiative et le bénévolat, peut au contraire encourager la solidarité aussi bien sur le plan symbolique que pratique, notamment en réduisant par des programmes de protection sociale les risques auxquels les individus sont confrontés et en favorisant l'acquisition de compétences afin que les individus puissent développer leur potentiel (DRHC, 1999). Les constats que font de Hart et Dekker (1999), Rothstein (1998) et Hall (1999) respectivement pour les Pays-Bas, la Suède et le Royaume-Uni ne corroborent pas I'hypothèse d'un étouffement de l'initiative par l'État-p rovidence. Ils observent, en effet, que les niveaux de bénévolat, de contacts informels et de participation à des projets d'intérêt collectif sont relativement élevés dans ces pays et qu'ils ne semblent pas avoir baissé du fait des politiques sociales.
... quant à l'Internet, ses effets ne se sont pas encore pleinement réalisés.

Le temps de travail et l'étalement des villes interviennent également.

On a suggéré que le système de protection sociale évince les activités associatives et le capital social, mais les preuves sont faibles. 
L'instabilité croissante de la vie familiale a pu dégrader le capital social.
Une enquête portant sur huit pays européens montre en particulier que deux des pays où la protection sociale est la plus développée, les Pays-Bas et la Suède, sont aussi ceux où le bénévolat est le plus important (Gaskin et Davis Smith, 1995)19. En réponse à la question : «Avez-vous, au cours de l'année écoulée, effectué pour ou dans une organisation qui n'a pas de lien avec votre travail rémunéré, un travail ou une activité non rémunéré qui ne vise pas exclusivement votre propre intérêt ou celui de votre famille ? », 36 pour cent de la population suédoise ont répondu oui, comparé à une moyenne de 27 pour cent pour les autres pays d'Europe.

Depuis la révolution industrielle, le rôle de la famille nucléaire ou élargie a fléchi aussi bien comme unité de production économique que dans sa mission d'éducation, ce qui a peut-être affaibli certains réseaux familiaux. Avec les changements intervenus à une date plus récente dans les structures de la famille et sa stabilité, les personnes ont plus tendance à faire appel à des liens impersonnels pour se procurer des financements, et pour assurer la garde et l'éducation de leurs enfants. Cela dit, la famille élargie, grâce à son capital culturel, peut encore constituer un important filet de sécurité en répond ant aux besoins matériels et financiers en périodes difficiles et en participant à la prise en charge des enfants et des personnes âgées (Abrams et Bulmer, 1986).

Selon des projections établies à partir de données récentes (State of A merica's Children, 1998 Yearbook Children's D efense F und), 1 enfant sur 2 va vivre à un moment ou à un autre dans une famille monoparentale, 1 sur 3 va naître hors mariage, 1 sur 4 va vivre avec un seul parent, 1 sur 8 naîtra d'une mère adolescente et 1 sur 25 ne vivra avec aucun de ses deux parents. Pour Fukuyama, l'éclatement de la famille, l'absence de l'un des parents ou des deux et la montée de l'individualisme sont des facteurs qui entament la confiance et contribuent à amplifier les dysfonctionnements sociaux. S'il est difficile de savoir dans quelle mesure à long terme ces changements vont favoriser la confiance et l'engagement civique, ils ont en tout cas des conséquences importantes sur le plan de la politique sociale, car avec la dissolution des liens familiaux et le vieillissement de la population, il y a un risque croissant d'isolement social des personnes démunies.

\subsection{Quelle est l'incidence du capital social sur le bien-être ?}

Des avantages divers découlent de niveaux plus élevés de liens sociaux.
A l'instar du capital humain, le capital social procure d'importants avantages aux ind ividus et aux sociétés. Certains de ces avantages, notamment un accroissement de la productivité, sont purement économiques et leur incidence sera passée en revue plus loin dans la section 3.9. La présente section porte, quant à elle, sur les effets du capital social sur d'autres aspects du bien-être.

Un certain nombre d'études menées dans divers pays ont montré que, compte tenu de l'état de santé initial, la densité des liens sociaux - autrement dit l'étroitesse des liens noués avec la famille, les amis et les connaissances - va de pair avec l'espérance de vie20. Putnam (2000a) passe en revue un grand nombre d'études empiriques qui concluent à un lien positif entre le capital social (essentiellement mesuré par rapport aux réseaux sociaux) et l'instruction, le bien-être des enfants, la délinquance, la vitalité des quartiers (par exemple prix de revente des logements), la santé (phy- 
sique et psychologique), le bonheur et le gouvernement démocratique. Putnam signale aussi une forte corrélation entre les mesures du capital social au niveau des différents états des États-Unis, et une mesure composite du bien-être des enfants, compte tenu de caractéristiques telles que la race, le revenu et le niveau de formation initial.

C'est peut-être dans le domaine de la santé individuelle que l'impact positif du capital social est le plus évident. Au 19e siècle déjà, le sociologue Emile Durkheim constatait un lien étroit entre le taux de suicide et le degré d'intégration des individus dans la société. II montrait en effet que les taux de suicide tendaient à s'élever dans les périodes de mutations rapides de la société. Cet effet était attribué à la désorganisation du tissu social et à l'affaiblissement des liens sociaux. Putnam (2000a) cite les résultats de nombreuses études récentes qui indiquent une corrélation entre les liens sociaux et la santé et le bien-être individuel (compte tenu des caractéristiques sociales, raciales et démographiques des individus). La longévité elle aussi paraît être influencée par l'importance des liens sociaux. II voit à cela deux raisons possibles : i) les réseaux sociaux fournissent une aide concrète, qui réduit le stress physiologique et physique et ii) le capital social déclenche peut-être un mécanisme physiologique qui stimule les systèmes immunitaires des individus et leur permet de lutter contre la maladie et d'atténuer le stress.

Les recherches consacrées aux effets du capital social sur la santé montrent que l'isolement social tend à précéder la maladie, ce qui renforce l'idée selon laquelle cette situation est une cause plutôt que la conséquence de la maladie. Les études de psychologie menées sur plus de 30 ans confirment le rapport entre des liens qui vous apportent un soutien et la santé mentale (Brown et Harris, 1978, par exemple). Putnam (2000a) passe également en revue des données relatives à l'impact du capital social sur le bien-être et le bonheur déclarés. Là encore, on constate un lien positif, même une fois éliminés les autres facteurs. Il observe que « ... dans toutes les enquêtes successives, les gens déclarent eux-mêmes que les bonnes relations familiales, amicales ou amoureuses - beaucoup plus que l'argent et la céléb rité - sont nécessaires à leur bonheur » (p. 332). Les personnes âgées qui vivent seules et n'ont pas d'amis ou de famille, encourent un risque relativement plus grand de démence ou de maladie d'Alzheimer, toutes choses égales d'ailleurs. Une enquête menée pendant trois ans sur 1200 personnes par le Centre de recherche en gérontologie de Stockholm a montré qu'entre autres facteurs, un réseau social important protège contre la démence (Fratiglioni et al., 2000). L'importance de contacts satisfaisants avec autrui, surtout avec la famille immédiate et les enfants, a été mise en lumière ; ils semblent protéger contre la démence, même s'ils sont relativement peu fréquents.

Rose (2000) a montré qu'en Russie les liens avec d'autres personnes en qui on peut avoir confiance ou sur lesquelles on peut compter pour obtenir de l'aide ou pour guider l'évolution de sa vie expliquent en grande partie les différences de santé physique et affective d'un individu à l'autre. L'effet du capital social est proche de celui du revenu du ménage et plus important que celui du niveau de formation. Rose constate la présence de solides réseaux informels d'amis, de parents et d'autres groupes proches, à côté d'une vie politique et sociale plus distante où il y a moins de confiance. II en conclut que les gens comptent plus pour traiter leurs pro-
Une corrélation existe entre le capital social et une meilleure santé...

... et l'isolement social est lié au malheur et à la maladie. 
Le capital social améliore le bien-être de l'enfant...

... diminue les taux de maltraitance des enfants...

... et facilite la transition à la vie d'adulte.

Il existe aussi une corrélation entre le capital social et une baisse de la délinquance. blèmes sur le capital social informel que sur les institutions formelles de l'État.

Putnam (2000a) fait état de données qui montrent que le capital social a presque autant d'impact que la pauvreté sur l'existence des enfants21. Si la pauvreté s'accompagne chez les adolescents de taux plus élevés de fécondité, de mortalité et d'oisiveté, l'engagement dans la collectivité a l'effet op posé. En revanche, le niveau d'instruction de la population adulte a beaucoup moins d'effet sur le bien-être des enfants, une fois éliminés les facteurs pauvreté, capital social et caractéristiques démographiques. Toutefois, étant donné les impacts de l'éducation sur la pauvreté et le capital social, ces résultats ne rendent peut-être pas bien compte de l'effet interactif ou conjugué qu'exerce le capital humain, notamment au travers du capital social et de la situation socio-économique.

Les travaux des psychologues montrent que les taux de maltraitance des enfants tendent à être plus élevés dans les quartiers où il y a moins de cohésion (Korbin et Coulton, 1997). Garbarino et Sherman (1980), étudiant deux quartiers où les niveaux de revenu, les taux d'activité des femmes et la proportion de ménages monoparentaux sont analogues, constatent que les habitants des quartiers où le risque de maltraitance des enfants est plus élevé hésitent davantage à demander de l'aide à leurs voisins et que les parents ont moins tendance à se garder mutuellement leurs enfants ou à laisser leurs enfants jouer avec les autres. Dans les quartiers à faible risque, en revanche, les enfants ont trois fois plus de chances que ceux des zones à haut risque de trouver l'un des parents à la maison au retour de l'école.

Runyan et d'autres ont également constaté que les liens sociaux des mères étaient très importants pour éviter les problèmes comportementaux et affectifs des enfants ultérieurement. Par liens sociaux, on entend la présence de réseaux sociaux qui apportent un soutien aux mères aussi bien qu'une attitude positive de la société environnante. Ils concluent que : « le capital social des parents... profite à leurs enfants, de même que les enfants bénéficient du capital financier et humain de leurs parents » (Runyan et al., 1998). Les familles où les liens sont relativement étroits paraissent avoir plus de chances de faire respecter leurs valeurs. Inculquer la confiance au sein de la famille peut aider les jeunes pour leur passage à la vie adulte et leur pleine participation civique (Teachman, Paasch et Carver, 1999 ; Darling et Steinberg, 1997).

Le capital social joue un rôle important non seulement en décourageant les comportements antisociaux ou délinquants, mais aussi en valorisant les énergies collectives et en les canalisant dans un sens positif. Le rapprochement des données de la World Values Study et des résultats du Sondage international de victimisation (ICVS) 22 montre qu'un certain nombre de normes sociales et de valeurs qui font partie du capital social peuvent expliquer des différences entre les pays dans la proportion de victimes d'actes de délinquance. Halpern (à paraître) utilise pour 18 pays des données, recueillies dans le cadre de I'ICVS, qui établissent un lien entre certaines valeurs et l'incidence de la délinquance, tout en neutralisant les effets de l'inégalité, du PIB par habitant et de la confiance. II constate, ce qui n'est pas surprenant, une forte corrélation entre la délinquance et les attitudes « égoïstes » (dont témoigne la propension à conserver pour soi de l'argent trouvé, à tricher, à mentir et à resquiller dans les transports 
en commun). Ces attitudes sont plus fréquentes chez les jeunes, les hommes et les citadins. Deux tiers de la variation entre pays des taux de délinquance peuvent s'expliquer par des variables nationales de valeurs égoïstes, d'inégalité économique et de confiance sociale23. II résume ses résultats en disant que l'inégalité économique est le « motif », la confiance sociale «l'opportunité » et les valeurs d'intérêt égoïste les « moyens » de passage à l'acte délinquant. Ces résultats se rapportent à des données nationales à un moment donné et ne prouvent pas le lien de cause à effet, mais ils suggèrent qu'il existe, entre les aspects en question du capital social et la délinquance, des liens qui peuvent être importants au niveau global. Kawachi et al. (1997) pensent aussi que, dans la relation entre le crime, la méfiance sociale et l'inégalité un lien causal essentiel est le manque d'estime de soi, de dignité et de statut social. Lorsque l'estime de soi, la dignité et le statut social sont dégradés par la pauvreté et l'exclusion, cela se répercute sur la confiance et les liens sociaux, et des conséquences en découlent pour la santé et la délinquance.

Des observations faites aux États-Unis montrent que, même compte tenu de la pauvreté et d'autres facteurs susceptibles d'encourager les comportements criminels, les communautés qui se caractérisent par i) I'anonymat et le manque de contacts entre les résidents, ii) la présence de groupes d'adolescents non surveillés et iii) un faible niveau de participation civique locale, sont plus vulnérables à la criminalité et à la violence (Sampson et Morenoff, 1997, et Sampson, 1995). Putnam (2000a) soutient qu'aux États-Unis, la baisse du capital social dans les quartiers, c'est-à-dire des fonctions d'encadrement, de socialisation, de grade et d'organisation exercées par le milieu environnant est un facteur important de déclin des quartiers pauvres des grandes villes. Sampson, Raud enbush et Earls (1997) ont utilisé d'abondantes données d'études concernant les quartiers de Chicago pour montrer que la confiance mutuelle et l'altruisme de voisinage étaient des aspects primordiaux pour expliquer les différences de taux de criminalité entre quartiers (une fois prises en compte leurs caractéristiques socio-économiques). Leur analyse, qui complète l'observation de Putnam sur l'effet qu'a le capital social informel sur l'acquisition de connaissances (voir l'annexe C), laisse à penser que « la participation individuelle à des organisations locales, le nomb re de programmes axés sur le quartier et l'étendue des liens de parenté et d'amitié dans le quartier ne semblaient pas faire de différence marquée ». Les auteurs concluent plutôt que «la réduction de la violence semble plus directement attribuable à l'encadrement informel exercé par les résidents et leur cohésion » (Sampson, Raudenbrush et Earls, 1997, pp. 918-924).

Le capital social peut influer de manière cruciale sur l'efficacité avec laquelle les institutions publiques et les pouvoirs publics promeuvent l'insertion et la cohésion sociales. Putnam (1993 et 2000b) utilise des données sur le comportement des électeurs, la fraude fiscale et l'engagement civique et politique pour montrer qu'il existe un lien entre le capital social et la performance des institutions publiques. Les régions ou les états où les niveaux de confiance et d'engagement sont élevés ont en général une administration de meilleure qualité, même une fois pris en compte d'autres facteurs socio-économiques. En outre, les individus qui sont liés à d'autres personnes dans leur zone d'habitation, leur activité professionnelle ou une association se désintéressent moins souvent de l'activité politique locale et ont plus rarement des opinions extrêmes. En d'autres
Le capital social est lié à une amélioration de l'administration publique... 
... et joue peut-être un rôle même plus important que le capital humain pour améliorer le bien-être. termes, les associations civiques bénévoles développent les compétences sociales et civiques - ce que Putnam appelle «écoles de démocratie ». La plupart des formes d'engagement civique, mais pas toutes, aident à créer des relations de confiance, de réciprocité et de coopération qui ont des retombées pour les collectivités, les sociétés et les économies locales. Dans les associations civiques, on ap prend à discuter avec d'autres personnes, qui ont souvent un point de vue différent.

Comme on l'a vu dans le chapitre 2, un nombre croissant d'études porte sur les facteurs qui déterminent la satisfaction personnelle ou le bien-être subjectif. Utilisant des résultats d'enquêtes, ces études examinent l'association entre le niveau déclaré de bien-être ou de bonheur et des facteurs démographiques, sociaux, ethniques ou autres. Faute de données permettant de suivre les individus dans le temps, il est difficile de tirer des conclusions d'ordre général. Toutefois, un certain nombre de points intéressants se dégagent de ces études. Putnam (2000a) étudie les tendances des niveaux déclarés de bonheur et les caractéristiques individuelles qui y sont associées (p. 333). II découvre que, parallèlement à la santé personnelle, le facteur le plus significatif semble être les liens sociaux. La situation matrimoniale semble aussi étroitement liée au bonheur déclaré. Putnam constate qu'une augmentation des revenus et du capital humain et social semble entraîner une progression du bonheur tant individuel que collectif24. Le revenu moyen à l'échelon régional a beaucoup moins d'effet sur le bien-être que l'instruction, la santé ou le capital social. Toutes choses égales d'ailleurs, aux États-Unis une augmentation du revenu moyen dans l'état n'accroît pas le bonheur individuel déclaré alors qu'une augmentation du capital humain ou social a cet effet.

Les résultats d'études faites aux États-Unis et en Grande-Bretagne examinés par Blanchflower et Oswald (2000) confirment l'idée que les liens sociaux pourraient avoir sur le bonheur un effet supérieur à celui de l'instruction et du revenu, au moins pour les tranches de revenu moyenne et supérieure à la moyenne. Myers (1999) arrive à des résultats semblables pour les États-Unis au sujet de l'impact qu'ont les relations personnelles et les liens sociaux étroits sur le niveau déclaré de qualité de vie. Blanchflower et Oswald examinent l'impact sur le bonheur d'événements comme le mariage, le chômage, le divorce, etc. Comme Putnam (2000a), ils constatent que le bonheur a diminué entre le début des années 70 et la fin des années 90 . Bien que l'instruction ait une corrélation importante avec le bonheur, elle semble venir en troisième position après les liens sociaux et la santé. Le revenu compte aussi, mais moins que les liens sociaux, la santé ou l'instruction, et son effet diminue aux tranches de revenus supérieures. Ces résultats sont cohérents avec les rapports macro-économiques observés dans une comparaison entre pays (Inglehart, 1997) entre les niveaux de PIB par habitant et les niveaux globaux de bien-être.

\subsection{La relation entre le capital social et l'inégalité sociale}

Les formes aiguës d'exclusion sociale (en fonction du statut social, de l'appartenance ethnique, du sexe ou de la région) semblent aller de pair avec des niveaux plus faibles de confiance et d'engagement civique notamment intergroupes. Les groupes peuvent être moins disposés à 
coopérer avec d'autres groupes ou à leur faire confiance même lorsque la coopération et la confiance sont solides au sein de chaque groupe. Dans la présente section, nous traitons deux questions interdépendantes:

- A l'échelon micro-économique, quels liens y a-t-il entre le capital social et l'inégalité sociale (accès à l'instruction, aux emplois et aux revenus) ?

- A l'échelon macro-économique, le capital social entraîne-t-il une plus grande égalité des revenus et des chances dans la société, et cette égalité génère-t-elle une élévation des niveaux de confiance et de cohésion sociale?

Les pays et les régions où les niveaux de confiance et d'engagement civique sont élevés sont en général plus égalitaires en ce qui concerne les revenus, la maîtrise des savoirs fondamentaux (le niveau de " littératie ») par les adultes et l'accès à la formation continue. Plus la dotation initiale en capital social est élevée, plus il est probable que les individus l'enrichiront pendant toute leur vie. Les personnes qui sont intégrées à des réseaux ou à des collectivités donnés ont d'importants moyens d'accès aux ressources et à une assistance dont les «étrangers » sont en fait exclus. Hall (1999), par exemple, constate qu'il existe des différences ap préciables de capital social selon les groupes sociaux au Royaume-Uni (point qui est également développé dans Galland, 1999, en ce qui concerne la France). Hall a constaté que les membres des classes moyennes ont plus de chances d'adhérer fréquemment à de nouvelles associations, d'accumuler les adhésions au cours de leur vie et d'ap partenir à des réseaux sociaux étendus et divers. A l'inverse, les personnes issues de la classe ouvrière adhèrent en général à un moins grand nombre d'associations, souvent pour y accomplir des activités précises, et restent memb res pendant longtemps.

L'évolution du capital social examinée dans la section 3.6 peut refléter des changements dans la structure des inégalités économiques dans l'ensemble des pays et au sein de chacun d'eux. Selon Putnam (2000a, p. 360), la baisse du capital social auxÉtats-Unis est liée à l'inégalité croissance des revenus et de la richesse. Le lien de causalité peut opérer dans les deux sens car l'auteur fait également état de données révélant que l'inégalité et la diminution de l'engagement civique se renforcent en général mutuellement. Knack (1999) constate également une corrélation positive entre l'égalité de revenu et la confiance dans différents pays. Toutefois, Fukuyama (1999) adopte un point de vue assez différent soutenant que l'éclatement des familles et la diminution de la confiance qui en résulte en général expliquent dans une certaine mesure la progression de la pauvreté auxÉtats-Unis.

En tirant parti des données recueillies à l'échelon des états à l'occasion de l'Enquête sociale générale menée aux États-Unis, Kawachi et al. (1997) soutiennent que les inégalités de revenu diminuent le capital social et par là même conduisent à une progression des taux de mortalité (ainsi que des taux de criminalité). Outre le rôle de médiation du capital social, il existe naturellement des liens directs entre la pauvreté et la mauvaise santé. L'idée essentielle qui ressort de leur analyse est que la répartition des revenus à l'échelon des différents états compte davantage que le revenu moyen dans les résultats en matière de santé25. Ce point de vue est appuyé, entre autres, par Wilkinson (1996) selon qui les
Le capital social est lié à l'égalité, mais le sens dans lequel le lien de causalité s'oriente est incertain.

Les inégalités économiques peuvent être à la fois la cause et la conséquence des inégalités devant le capital social.

Les données sont incertaines au sujet des liens entre l'exclusion sociale, le capital social et les inégalités devant la santé. 
Le capital social peut avoir pour effet soit d'enraciner l'éclatement social, soit de passer outre les divergences.
Le capital social peut atténuer les écarts dans la maîtrise globale des savoirs fondamentaux ( « littératie ») ou les niveaux d'instruction. inégalités devant la santé au sein des pays ou des régions sont plus étroitement liées au degré d'inégalité qu'au niveau de revenu moyen. Halpern et Nazroo (2000) laissent également entendre que les personnes issues des minorités ethniques présentent une santé mentale et un bien-être personnel meilleurs lorsqu'elles vivent dans des régions où leur groupe est fortement concentré. Toutefois, Lynch et al. (2000) mettent en garde contre « des interprétations excessivement simplistes des liens entre le capital social, le développement économique, la politique gouvernementale et la santé ». En outre, Muntaner et al. (2000) estiment que l'utilité de la notion de capital social du point de vue de la santé publique a peut-être été exagérée dans la mesure où ce concept a été défini dans une optique étroite et où il est focalisé sur une évaluation relativement optimiste de son lien avec la santé de la population. En conséquence, selon ces détracteurs, il faut considérer avec prudence la conceptualisation et les données empiriques limitées sur lesquelles repose le capital social.

Les pays à forte polarisation sociale, ethnique ou linguistique (Collier, 1998; Knack et Keefer, 1997) sont presque inévitablement confrontés à un risque plus grand d'éclatement social. Toutefois, nombreuses sont les sociétés multiraciales et ne parlant pas la même langue qui ont prouvé qu'elles étaient capables de gérer la diversité et de l'exploiter à des fins positives. Établissant une comparaison de différents pays, La Porta et al., par exemple, ne constataient aucune corrélation significative entre I'hétérogénéité ethnolinguistique et la méfiance déclarée.

Bien que l'« éclatement » des relations sociales puisse aboutir à la formation de nouvelles structures et de nouveaux réseaux, qui devraient être bienvenus, les nouvelles formes de capital social risquent d'être inégalement réparties. Si l'effet de l'Internet peut être source d'espoir et de motivation, il faut le considérer en regard des risques qu'il comporte : l'inégalité sociale d'accès au cyberespace, la nature impersonnelle apparente de ce moyen de communication, la nouvelle fragmentation des populations en des groupes distincts, enfin la possibilité qu'il offre d'être un outil de divertissement personnel et passif. Ces risques pourraient être accentués à l'avenir dans la mesure où certains groupes auront probablement accès à des technologies et des formes d'apprentissage nouvelles liées à l'amélioration des possibilités d'emploi.

A partir de données canadiennes sur les résultats des élèves, Willms (2001) conclut que les personnes qui viennent de milieux démunis et vivent dans des communautés défavorisées risquent tout particulièrement d'avoir un faible niveau d'instruction. Les communautés qui ont un fort capital social et culturel peuvent atteindre un niveau d'instruction plus élevé. Willms souligne l'importance que la discipline, la participation et les attentes des parents revêtent dans l'élévation des niveaux d'instruction et de littératie. En outre, la famille et la collectivité jouent un grand rôle dans l'amélioration des savoirs et savoir-faire de base des adultes désavantagés. D'après ces constatations, les mesures que les sociétés prennent pour remédier au handicap social sont un facteur déterminant de leur niveau global de maîtrise des compétences de base. Dans les différents pays, les écarts de niveau de compétences et de qualification des adultes sont plus grands parmi les personnes issues de milieux défavorisés. Cela donne à penser que, pour améliorer les normes globales à cet 
égard, il est essentiel de repérer les besoins des classes sociales défavorisées et surtout des personnes qui n'ont qu'un accès réduit à des réseaux sociaux.

\subsection{Incidence du capital social sur le bien-être économique}

\subsubsection{Productivité dans les entreprises et les organisations}

Toutes transactions, qu'elles soient privées, sociales, économiques ou politiques, reposent sur la confiance. La notion de réseaux est au cœur de la définition du capital social26. Les entreprises peuvent tirer parti des normes de coopération et confiance concrétisées par les réseaux intraentrep rises ou interentrep rises car ces derniers facilitent la coordination et permettent une diminution des coûts de transaction imputables aux activités de négociation et d'application des règles, à une information insuffisante et à une bureaucratie inutile27. Dans cette optique, la confiance prend de nombreuses formes et consiste notamment à croire que les autres ont de bonnes intentions, qu'ils sont compétents et fiables.

Humphrey et Schmitz (1998) soulignent que « des relations fondées sur la confiance entre agents économiques sont considérées comme faisant partie de l'avantage comparatif des entreprises manufacturières en Allemagne, au Jap on et dans certaines parties de l'Italie... ». Fournisseurs et acheteurs peuvent maintenir des relations à long terme de coopération et d'obligation mutuelle à mesure que se succèdent des transactions reposant sur la confiance et la constitution de réseaux. Les réseaux d'entreprises s'occupant de commercialisation, de formation ou de recherche peuvent procurer des avantages à long terme en réduisant les frais généraux, en mettant en commun l'information et en appliquant des sanctions en cas de comportements op portunistes. Dans certains créneaux, comme le développement de logiciels ou l'industrie du vêtement, les chefs d'entrep rise peuvent gagner du temps, en partageant l'information et en s'adaptant rapidement aux exigences des clients (Uzzi, 1996 et 1997).

Certains chercheurs ont étudié la notion de confiance généralisée par rapport à celle de confiance spécialisée. La Porta et al. (1997) constatent une association positive entre les recettes des vingt plus grosses entreprises, exprimées en pourcentage du PIB, et la confiance envers autrui en général, mais une association négative entre cette variable et la confiance envers la famille. Leur hypothèse est que les grandes entreprises réussissent peut-être dans les sociétés confiantes où les sanctions et punitions imposées aux comp ortements opportunistes sont moins nécessaires. En revanche, selon la même hypothèse, il serait plus difficile de créer de grandes entreprises dans des sociétés fermées reposant sur des liens familiaux ou ethniques. Dans ce modèle, la confiance tend à être un facteur exogène façonné par l'histoire et la culture, qui sert à faciliter l'action collective et la coopération, et renforce notamment l'engagement civique et l'efficacité gouvernementale.

De même, les réseaux intra-entrep rises et les normes de coopération peuvent aussi faciliter le travail en équipe, renforcer l'efficience et la qualité et améliorer les flux d'information et de savoir. Shimada (1988) fait observer que la coopération entre les travailleurs et la direction est la rai-
La confiance a un rôle à jouer par rapport à la productivité...

... lorsqu'elle est ancrée dans la culture de l'entreprise...

... et peut aboutir à un agrandissement et à une plus grande efficacité des unités de production...

... ainsi qu'à une coopération accrue au sein des entreprises. 
son essentielle de la compétitivité (traditionnellement) forte des constructeurs automobiles japonais : «Dans une entrep rise américaine, chaque salarié tient à assurer sa propre réussite et n'est pas disposé à transmettre son savoir à son collègue. Le salarié japonais, en revanche, est prêt dans toute la mesure du possible à le faire car il est convaincu de pouvoir réussir non tout seul mais uniquement dans le cadre d'une équipe » (Omori, 2001). Les différents types de capital social et organisationnel peuvent être plus ou moins adaptés selon le stade de développement économique - en témoigne la baisse de compétitivité des constructeurs automobiles japonais dans les années 90 .

\subsubsection{Productivité dans les régions et les quartiers}

Le capital social peut faciliter les systèmes régionaux d'innovation.

L'accès au capital social aide à trouver un emploi.
Les systèmes industriels régionaux fondés sur des réseaux locaux ont le potentiel d'être plus souples et plus dynamiques que ceux où l'acquisition de connaissances n'a lieu que dans des entreprises individuelles. Les réseaux régionaux ou locaux peuvent favoriser la circulation de l'information, l'apprentissage mutuel et des économies d'échelle. Putnam (2000a) compare aux États-Unis l'effet de la Silicon Valley et celui de la Route 128. Dans la Silicon Valley en Californie, un groupe de chefs d'entreprises, aidé par les chercheurs des universités locales, a contribué au développement d'un centre mondial de technologie de pointe. Putnam note que : «La réussite est en grande partie attribuable aux réseaux horizontaux de coopération, officielle ou non, qui se sont établis entre les nouvelles entreprises de la région. » A l'inverse, sur la Route 128, à l'extérieur de Boston, l'absence de capital social interentreprises a conduit à une forme plus traditionnelle de hiérarchie des entreprises caractérisée par la culture du secret, l'autonomie et le principe de la «chasse gardée».

\subsubsection{Recherche d'emploi}

Barbieri, Russell et Paugam (1999) montrent que dans un certain nombre de pays européens, le capital social est une ressource précieuse pour trouver un emploi, surtout dans des marchés du travail ouverts et souples. Plus que sur le nombre de liens sociaux auxquels un individu peut faire appel, le succès de la recherche d'emploi dépend de la diversité des personnes avec lesquelles cet individu a des contacts et sur lesquelles il peut compter. Les liens d'accointance sont plus importants que les liens d'attachement pour les personnes qui cherchent un emploi. Or, les chômeurs ont moins accès à des réseaux et à des contacts étendus associés au milieu de travail.

Un certain nombre d'économistes dont Granovetter (1973) ont insisté sur le rôle des relations occasionnelles ainsi que des amis proches et de la famille pour trouver un emploi. De même, Burt (1992) souligne que l'absence de liens étroits 28 est un facteur qui encourage la mobilité des individus et le partage des connaissances. Dans les réseaux fermés ou denses, l'information est plus souvent répétitive. Selon cet auteur, l'information et l'influence (sur sa propre autonomie et celle d'autrui) sont deux types d'avantages attribuables aux réseaux sociaux. Un troisième type d'avantages prend la forme de solidarité sociale mettant en jeu coopération, sens du devoir social et réciprocité sans exigence de «compensation » immédiate pour 
ceux qui contribuent au bien-être des autres. Loury (1987), I'un des « inventeurs » de la notion de capital social, a créé ce concept pour signaler le fait que même si l'on annulait les avantages humains et financiers dont bénéficient les blancs en Amérique, la richesse de leurs liens avec les grandes institutions américaines les avantagerait par rap port aux membres des classes moyennes appartenant à une minorité.

\subsubsection{Avantages macro-économiques}

Comme Arrow (1972) l'a fait observer, la confiance entre les personnes et les normes de confiance à l'égard des institutions peuvent être primordiales dans de nombreuses activités économique et sociales. Toutefois, le rôle que le capital social joue dans l'explication des écarts de croissance économique d'un pays à l'autre n'a guère été analysé à ce jour, ce qui reflète la relative nouveauté de ce concept, son hétérogénéité, et la difficulté rencontrée pour recueillir des indicateurs ad équats à l'échelle internationale. De plus, de sérieux problèmes de mesure se posent au stade de l'agrégation.

Les résultats témoignant de l'effet macro-économique du capital social varient selon les pays choisis, la période retenue et l'intégration ou non d'autres variables explicatives dans les équations de croissance. Utilisant les données provenant de la World Values Study, Knack et Keefer (1997) ont constaté une corrélation positive entre une mesure de la confiance générale interpersonnelle et l'accroissement du PIB, une fois neutral isé l'effet du revenu initial par habitant, d'une variable représentant le capital humain et du prix relatif des biens d'équipement 29 . Utilisant la même source de données, Hjerrpe (1998) a également découvert un lien positif et significatif entre la confiance et la croissance économique pour un échantillon de 27 pays (dont un certain nombre de pays à revenu intermédiaire ou faible)30, une fois éliminé l'effet du capital physique, de l'ouverture aux échanges et de la proportion d'adultes ayant fait des études supérieures. Cela étant, Helliwell (1996) a constaté une corrélation négative entre la confiance et l'accroissement de la productivité totale des facteurs dans un échantillon de 17 pays Membres de I'OCDE.

D'autres chercheurs ont laissé entendre que la confiance peut stimuler l'épargne, la prise de risque et l'investissement. Guiso et al. (2000) signalent que dans les régions d'Italie où la confiance sociale est élevée, les ménages investissent moins en espèces et davantage en actions, utilisent plus de chèques, ont davantage accès au crédit institutionnel et moins recours au crédit informel. Les entreprises ont aussi davantage accès au crédit et ont plus souvent des actionnaires multiples. C'est lorsque les forces de l'ordre sont le plus faibles et parmi les groupes de population peu instruits que l'effet de la confiance est le plus fort. Le comportement financier des personnes originaires d'une autre région est surtout influencé par le niveau de confiance existant dans le milieu où elles vivent et dans une moindre mesure par le niveau de confiance existant dans la province d'où elles viennent.

Les analyses effectuées au niveau infranational peuvent être plus révélatrices car elles tiennent compte implicitement de certains facteurs de confusion, d'ordre contextuel et culturel. L'Italie du nord marque de
Les données récentes témoignant de l'incidence de la confiance sur la croissance économique sont constratées... 
... tout comme le sont les éventuels liens entre l'adhésion à des groupes et la croissance économique.
D'autres recherches donnent à penser que l'infrastructure sociale joue un rôle important dans la croissance économique. net progrès en matière de gestion des affaires publiques, de performances des institutions et de développement économique, d'autres facteurs étant alors éliminés31. Putnam (1993) ainsi que Helliwell et Putnam (1999a) affirment qu'un capital social plus solide a permis à la région du nord de tirer parti de la réforme régionale pour progresser plus rapidement que le sud. Pour asseoir leurs arguments, ces auteurs ont entrep ris de comparer l'Italie du nord et l'Italie du sud : ils ont découvert un rapport entre la performance interrégionale depuis le milieu des années 80 et l'amélioration de la satisfaction croissante des citoyens à l'égard des autorités régionales enregistrée dans le nord du pays à la suite des réformes réalisées dans les années 7032. Jusqu'au début des années 80 , les niveaux de PIB réel par habitant des différentes régions de l'Italie avaient tendance à converger. Depuis lors, les écarts d'une région à l'autre se sont de nouveau creusés.

On ne connaît pas de façon précise le lien existant à l'échelle nationale entre les niveaux d'adhésion à des groupes et la croissance économique. Putnam (1993) a découvert un lien entre les niveaux d'engagement civique et le développement économique sur une longue durée. Il en conclut que dans les interactions quotid iennes structurées par des associations civiques, les gens ap prennent la confiance, les normes sociales et forgent des réseaux efficaces d'action publique. Les associations civiques peuvent créer un réseau horizontal dense et offrir de nombreuses occasions d'acquérir le capital social que constitue la confiance.

D'autres études ont abouti à des résultats peu concluants, ce qui donne à penser que les variables représentatives ne rendent peut-être pas compte de certains aspects importants du capital social. Knack et Keefer (1997), utilisant des indicateurs tirés de la World Values Study pour le nombre de personnes appartenant à des groupes, ont constaté que les associations dont les fonctions tend ent à défend re les intérêts de leurs membres - notamment les syndicats, les parties politiques et les associations professionnelles - avaient peu de rapport avec la croissance ou les taux d'investissement. Ils ont également observé que beaucoup d'autres types d'association tels que les organisations religieuses, éducatives, artistiques, musicales ou culturelles, n'avaient pas de corrélation avec la croissance économique, mais avaient une corrélation négative avec l'investissement.

II n'est donc pas évident que les changements enregistrés dans l'appartenance à des groupes ou dans d'autres formes d'interactions sociales aient influé sur les écarts récents de croissance économique entre les pays de I'OCDE. Ainsi, la baisse des taux d'adhésion à des groupes et de participation civique enregistrée aux États-Unis va de pair avec des augmentations rapides du revenu par habitant.

Une grande partie des travaux récents ont porté sur les déterminants à court terme de la croissance économique. D'autres recherches donnent à penser que les pays atteignent des niveaux de production par travailleur plus élevés à long terme, s'ils associent des taux élevés d'investissement dans le capital physique et humain et une infrastructure sociale 33 de qualité (voir Hall et Jones, 1999). La qualité de "l'infrastructure sociale » est essentiellement liée à l'efficacité des institutions et des politiques gouvernementales qui constituent l'environnement économique dans lequel les particuliers 
et les entrep rises effectuent des investissements, créent et transfèrent des idées et produisent des biens et des services. Cette notion d'infrastructure sociale est très proche de celle de conditions politiques, institutionnelles et juridiques mentionnées dans le chapitre 1.

Il faut être prudent lorsqu'on interprète le rôle de la confiance dans les équations comparant la croissance économique de plusieurs pays. II est fort possible que la confiance soit déterminée par d'autres aspects des sociétés qui ne figurent pas dans les analyses de régression de la croissance et qu'elle ait une corrélation avec eux. Il se peut, par exemple, que la corruption ou une application insuffisante de la loi affaiblissent la confiance et, pour des raisons totalement indépendantes, le taux de croissance. Comme le notent Knack et Keefer (1997), la confiance pourrait même être attribuable à l'optimisme de sociétés qui ont de bons résultats économiques. La pauvreté et le sous-développement économique peuvent freiner l'établissement de la confiance car les gens qui vivent dans une société d'insécurité et de sous-développement économiques sont sans doute moins incités à faire confiance aux autres.

L'effet exercé sur les performances macro-économiques peut aussi être en partie indirect et résulter d'un taux d'investissement plus élevé dans le capital physique et humain et d'une meilleure performance de I'«infrastructure sociale » (ou des conditions politiques, institutionnelles et juridiques, selon le chapitre 1). La Porta et al (1997)34 ainsi que Knack et Keefer (1997) citent des données montrant que la confiance est associée à certains indicateurs de performance gouvernementale, dont l'efficacité du système judiciaire et la qualité des fonctionnaire. Toutefois, ces études montrent qu'il n'existe qu'un faible rapport entre la confiance et la croissance économique (sur la période 1970 à 1993), mais qu'il y a un lien plus fort entre des résultats très divers comme le niveau d'instruction et l'investissement dans le capital matériel, une fois pris en compte un certain nombre de facteurs. Elles révèlent également une corrélation positive entre les niveaux d'instruction et la confiance, toutes choses égales d'ailleurs 35 .

\subsection{Conclusion}

C'est avec prudence qu'il faut dégager des conclusions des études qui reposent sur des mesures représentatives trop globales ou indifférenciées du capital social. II ressort toutefois de ce chapitre que d'après une large gamme d'études empiriques réal isées dans un certain nombre de pays, le capital social procure probablement des avantages économiques, sociaux et personnels positifs.

II existe également une relation bilatérale entre le capital social et le capital humain. On observe une association positive même à l'échelon international entre, par exemple, l'engagement civique et la confiance d'une part et les niveaux de formation d'autre part36.

Il est plus difficile de faire apparaître clairement un lien entre le capital social et la croissance économique. Comme dans le cas du capital humain, on se heurte au problème de la qualité et de l'amplitude des mesures indicatives, de la complexité des interrelations entre divers
Il importe de bien comprendre les limites des recherches.
En conclusion, le capital social semble procurer des avantages économiques et sociaux...

... et le capital humain et le capital social pourraient se renforcer mutuellement.

Certes, la recherche donne matière à réfléchir, mais aucune donnée fiable ne permet pour l'instant de dire 
que le capital social est généralement lié à la croissance économique.

Les difficultés d'accès au capital social sont aussi à l'évidence un aspect important de l'exclusion sociale. déterminants et de la difficulté de faire des comparaisons entre des pays dont les traditions culturelles, institutionnelles et historiques sont très différentes. Comme l'a laissé entend re Temple (2001), il serait fructueux d'entreprendre des recherches complémentaires dans ce domaine.

Les données examinées dans ce chapitre, témoignant des avantages que procure l'accès au capital social, sont assez remarquables pour dire que le capital social est un aspect à prendre en considération dans les politiques de lutte contre la pauvreté et l'exclusion sociale - en réalité, l'expression d'exclusion sociale implique l'impossibilité d'accéder au capital social. 


\section{Notes}

1. De Tocqueville (1835) écrit : «Les Américains combattent l'individualisme par la doctrine de l'intérêt bien entendu » ajoutant par exemple, qu' « ils montrent complaisamment comment l'amour éclairé d'eux-mêmes les porte sans cesse à s'aider entre eux et les dispose à sacrifier volontiers au bien de l'État une partie de leur temps et de leurs richesses ».

2. Pour Durkeim, la société est composée d'« organes » (faits sociaux), ou de structures sociales qui exercent diverses fonctions dans la société.

3. Weber s'intéresse aux individus et aux schémas et régularités des actions. II s'intéresse surtout aux actions qui font clairement intervenir, entre le stimulus et son ultime réponse, des processus de réflexion (qui leur donnent du sens).

4. Jacobs (1961) assimile dans sa définition le capital social à des « réseaux de proximité ».

5. La définition du capital social retenue de préférence par la Banque mondiale est la suivante : «Le capital social désigne les institutions, relations et normes qui déterminent la qualité et la quantité des interactions sociales d'une société. De plus en plus d'éléments factuels montrent que la cohésion sociale est capitale pour que les sociétés prospèrent économiquement et pour que le développement soit durable. Le capital social n'est pas simplement la somme des institutions d'une société - c'est le ciment qui les lie. » (http://www.worldbank.org/poverty/scapital/whatsc.htm)

6. Certains auteurs y ajouteraient les « croyances ». Adler et Kwon (2000), par exemple, les définissent comme des visions, interprétations et significations stratégiques communes. Les « croyances » sont proches des valeurs qui sous-tendent la coopération.

7. Putnam (1993) donne l'exemple des «membres des chorales florentines, qui viennent à la chorale parce qu'ils aiment chanter et non parce que leur participation renforce le tissu social toscan ».

8. II ne faut pas déduire de ces exemples que tous les groupes unis par des liens forts ont un impact social négatif. Certaines formes de liens familiaux ou ethniques, par exemple, peuvent avoir sur la santé et l'emploi des effets positifs qui entraînent des retombées pour d'autres groupes.

9. Les formes exclusives de liens d'accointance peuvent comprendre des formes d'idéologies extrêmes ou totalitaires.

10. Cette enquête regroupe des enquêtes nationales représentatives effectuées sur les valeurs et croyances essentielles du public dans plus de 65 sociétés. Elle prolonge les enquêtes sur les valeurs européennes (European Values Surveys) dont la première série a été réalisée en 1981-89. Une deuxième série d'enquêtes, conçues au niveau mondial, a été effectuée en 1990-1993, une troisième en 1995-1997, et une quatrième est en cours en 1999-2000. Pour plus de précisions, voir http://wvs.isr.umich.edu/index.ht/m.

11. Les mesures de la confiance semblent assez stables dans le temps pour les vingt pays pris en compte dans l'enquête en 1981 et 1990 (avec un coefficient de corrélation de 0.91).

12. Cette corrélation ne peut être attribuée à une confusion possible introduite par un niveau plus élevé de revenu par habitant : si l'on élimine l'effet du revenu par habitant, la corrélation partielle entre la confiance et la proportion de porte-monnaie rendus est supérieure à la corrélation simple.

13. Une certaine prudence s'impose dans l'examen de ces résultats car une forte corrélation existe entre le fait de vivre dans une famille monoparentale et de nombreux autres indicateurs de handicap. Par exemple, Joshi et al. (1999) ont constaté que les enfants de mères isolées ayant rompu toute relation avec le père de l'enfant ne s'en sortent pas plus mal sur le plan de leurs études ou de leur comportement que d'autres enfants lorsqu'on ne tient pas compte du revenu actuel ou du niveau de formation de la mère. 
14. Loury s'intéresse essentiellement à l'inégalité du capital social et au fait qu'il est à même d'influer sur la structure socio-économique de classes.

15. C'est la définition utilisée par la Banque mondiale sur son site http://www.worldbank.org/poverty/scapital/sources/civil1.htm.

16. Picciotto (1998) note que : «La discrimination fondée sur le sexe nuit à la confiance et aux relations familiales, restreint les réseaux sociaux, et appauvrit le capital social qui permet aux sociétés d'avancer en direction d'objectifs communs ».

17. De Hart et Dekker constatent que ceci est vrai indép endamment du niveau d'instruction, du sexe, de l'âge ou du niveau de revenu de la personne interrogée.

18. Cela dit, les femmes sans emploi sont plus engagées que les femmes employées à temps complet.

19. Les autres pays où le bénévolat est important sont l'Allemagne, la Belgique, la Bulgarie, I'Irlande, la Slovaquie et le Royaume-Uni.

20. Toutefois, les liens sociaux, indépendamment de leurs formes et de leur densité, n'ont pas toujours des effets positifs sur la santé : certains peuvent avoir un caractère oppressif ou impliquer un comportement mauvais pour la santé.

21. Cela reste vrai même lorsqu'on neutralise les facteurs que sont le revenu, le niveau d'instruction, la composition raciale et la structure familiale pour chaque État américain.

22. L'ICVS consiste à recenser les victimes de tous types de délinquance, qu'ils aient ou non fait l'objet de plaintes. Ces enquêtes ont été effectuées en 1989, 1992 et 1996. Voir http://ruljis.leidenuniv.nl/group/jfcr/www/icvs/ind ex.htm

23. Un résultat curieux de cette étude empirique est qu'à la confiance sociale correspondent des niveaux de délinquance plus élevés, si l'on élimine les facteurs d'inégalité et d'intérêt égoïste. On peut penser que cela signifie que, pour un niveau donné d'inégalité et d'intérêt égoïste, il existe une offre de « victimes » : les victimes continuent de faire confiance à autrui en ne fermant pas leurs portes ou leurs voitures malgré le comportement malhonnête des autres.

24. Putnam (2000a) ne découvre au niveau local (du comté des États-Unis) aucun impact du capital social sur le bonheur bien qu'un effet individuel existe toujours. Cela peut tenir à une erreur de mesure associée au fait que les échantillons sont plus petits.

25. On trouvera dans Lynch et al. (2000) une autre vision du rôle de médiation que joue le capital social dans l'impact de la pauvreté et de l'inégalité sur la santé. Ils affirment que l'impact du capital social sur la santé a peut-être été exagéré.

26. «Lorsque les gens sont confiants et fiables et lorsqu'ils ont des contacts répétés avec d'autres citoyens, les transactions commerciales et sociales courantes sont moins coûteuses » (Putnam, 2000a, p. 288).

27. Fukuyama (1999) définit un réseau comme « un groupe d'agents individuels qui partagent des normes ou valeurs informelles en dehors de celles qui sont nécessaires aux transactions commerciales ordinaires. »

28. Que Burt appelle les trous structurels.

29. Knack et Keefer concluent qu'une variation d'un écart-type de l'indice de confiance est associée à un changement du taux de croissance de 0.56 d'un écart-type. En d'autres termes, un niveau de confiance supérieur de 10 points de pourcentage (un peu moins d'un écart-type) est associé à un taux de croissance annuel supérieur de 0.8 point de pourcentage.

30. Parmi les pays non membres de l'OCDE peuvent être cités l'Argentine, le Brésil, le Chili, la Chine et la Russie.

31. Putnam a utilisé des séries chronologiques générales pour vingt régions de l'Italie pour la période allant de 1960 au milieu des années 80.

32. Le capital social est mesuré en fonction des éléments suivants : le pourcentage d'habitants qui lisent le journal, le nombre d'associations sportives et culturelles, le pourcentage de votants au référendum, l'incidence des systèmes de vote préférentiels et l'efficacité des autorités régionales.

33. L'infrastructure sociale d'un grand nombre de pays en développement est mesurée en fonction des éléments suivants : i) institutions favorables à la production plutôt qu'aux transferts ; ii) ouverture aux échanges internationaux; iii) primauté du droit et respect des droits de propriété ; iv) présence d'une langue intemationale ; et v) distance de l'équateur. 
34. Les auteurs constatent que dans l'ensemble des pays une progression d'un écart-type dans la même mesure de la confiance augmente l'efficience judiciaire de 0.7 d'un écart-type et réduit la corruption de $0.3 \mathrm{~d}$ 'un écart-type.

35. Knack et Keefer relèvent une forte corrélation $(r=0.83)$ entre la confiance et une estimation du nombre moyen d'années de scolarité pour 1980 et notent que « l'éducation peut renforcer la confiance et les normes civiques, par exemple si l'ignorance cond uit à la méfiance ou si l'éducation réduit les incertitudes concernant le comportement des autres ou si les élèves/étudiants apprennent à se comporter de manière coopérative » (p. 1270).

36. Ainsi, la progression de la demande d'éducation liée à l'évolution des conditions du marché du travail ne va pas nécessairement s'accompagner d'une augmentation du capital social. 



\section{Chapitre 4}

\section{CONSÉQUENCES POUR L'ACTION DES GOUVERNEMENTS ET AUTRES RECHERCHES À EFFECTUER}

« Aussi difficile soit-il de concevoir et d'évaluer l'instruction, nous disposons à cette fin de véritables outils. Les ministres de l'É ducation existent : des missions et des budgets leur sont confiés. M ais supposez que vous soyez ministre du capital social, quelles seraient vos fonctions au jour le jour ? Le capital social est en soi un concept hybride ». Helliwell (2001)

\subsection{Introduction}

II ressort du rapport que les décideurs devraient s'intéresser au capital humain et au capital social principalement pour quatre raisons :

1. Des données fiables montrent que le capital humain est un déterminant important de la croissance économique et, si l'effet du capital social sur la croissance n'est pas prouvé, les éléments dont on dispose suffisent pour qu'il soit justifié de consacrer des recherches complémentaires à cette éventualité.

2. On constate que le capital humain et le capital social sont associés à une large gamme d'avantages non économiques, dont l'amélioration de l'état de santé et un sentiment plus grand de bien-être. Ces observations revêtent une importance particulière eu égard au fait que ces dernières années le bien-être déclaré n'a pas en moyenne progressé au même rythme que le PIB.

3. Le capital humain et le capital social peuvent se renforcer mutuellement.

4. D'après ce qui précède, le capital humain et le capital social entrent pour une telle part dans les chances que chaque individu a dans la vie que les mesures prises pour favoriser l'intégration sociale doivent tenir compte de ces deux types de capital.

Le capital humain et le capital social permettent aux individus, aux collectivités, aux entreprises et aux sociétés, de faire face aux nécessaires et rapides mutations sociales et économiques - apprendre aux enfants à s'adapter à un monde en pleine évolution, reconvertir les adultes à de nouveaux types d'activité professionnelle et instaurer la confiance ainsi qu'une détermination commune dont dépendent la plupart des activités sociales et économiques. Ce capital représente une ressource essentielle pour le développement durable.
Le capital humain et le capital social contribuent à la fois à la croissance et au bien-être. 
Développer le capital humain et le capital social est une tâche qui incombe à chacun et chacune - et certainement pas aux seuls pouvoirs publics.

Il faut inscrire les politiques en faveur du capital social dans leur contexte.

Ce rapport laisse entrevoir quelques possibilités d'action pour développer le capital humain.
S'il est vrai que les établissements d'enseignement et les organismes de formation jouent un rôle important dans le développement du capital humain, leur réussite dépend de façon décisive du soutien plus vaste dont ils bénéficient de la société. Le capital humain et le capital social sont créés, de façon formelle et informelle, sur le lieu de travail, dans les communautés locales et au sein des familles. Le capital social réside dans les liens sociaux noués volontairement, ce qui implique que les pouvoirs publics souvent facilitent ou favorisent son développement, plutôt qu'ils ne le créent de façon active. De plus, le capital social et le capital humain se renforcent mutuellement. Pour toutes ces raisons, les idées de fond avancées dans ce rapport s'adressent non pas seulement aux pouvoirs publics mais également à tout un éventail d'autres acteurs et institutions, notamment les employeurs et les personnes travaillant dans des organisations bénévoles ainsi que dans des secteurs publics clés. Chacun a un rôle à jouer.

\subsection{Les actions en faveur du capital humain}

Les actions en faveur du capital humain passent par la reconnaissance d'un certain nombre de faits :

- L'évolution économique - en particulier la technicité accrue de l'activité économique - pourrait modifier la demande de capital humain, notamment en mettant davantage en vedette certaines qualifications non cognitives telles que l'aptitude à travailler en équipe et l'innovation 1 .

- Tous les environnements d'ap prentissage - dont la famille, le lieu de travail et les milieux préscolaires - sont importants.

- Le partenariat et le dialogue - entre les secteurs privé, public et associatif - sont essentiels tout comme l'est la coord ination des politiques - qui établit un lien entre les actions menées dans le domaine de l'éducation et les politiques de l'emploi et de la protection sociale.

- Des différences considérables existent du point de vue à la fois de l'accès et de la participation à des activités de formation complémentaire. Les politiques de formation tout au long de la vie doivent tenir compte de ces variations afin de venir à bout avec succès de l'exclusion sociale.

- Les profils temporels en jeu sont souvent longs, aussi bien pour le développement que pour l'érosion du capital humain et social.

Si l'on considère les travaux de recherche sur le capital humain, passés en revue dans ce rapport, en se plaçant dans la perspective d'autres étud es et d'autres travaux sur l'élaboration des politiques gouvernementales, certaines conséquences directes en découlent pour l'action des pouvoirs publics et certaines options méritent une réflexion plus poussée :

1. Les investissements dans le capital humain génèrent des avantages privés et sociaux non négligeables. Les toutes dernières recherches renforcent 
l'idée selon laquelle le capital humain joue un rôle notable dans la croissance économique. De plus, de nombreuses données montrent que l'investissement dans le capital humain procure des avantages non économiques appréciables - une meilleure santé, une amélioration du bien-être, des parents sachant mieux élever leurs enfants et un engagement social et politique plus grand. L'évolution de la structure de l'économie entraîne également un accroissement de la demande de qualifications dans la mesure où l'activité économique est plus axée sur le savoir et où la compétitivité des entreprises et des nations dépend de plus en plus du capital humain. II ne faut pas déduire de ces constats que tous les investissements dans l'éducation sont souhaitables, mais ces éléments corroborent effectivement l'idée selon laquelle les investissements dans la formation de capital humain, s'ils sont judicieusement ciblés, représentent un choix d'action raisonnable.

2. Le capital social est lié au niveau de formation, ce qui donne à penser que les divers réseaux locaux pourraient contribuer à la plupart des types d'activité de formation. Il faut établir des partenariats pour préparer et aider les personnes à ap prendre, étant entendu que cette préparation et cette aide sont souvent en grande partie apportées par le milieu familial2.

3. Les incitations à apprendre en permanence doivent être développées. II y a peut-être lieu de reconsidérer l'organisation des possibilités de formation offertes tout au long du cycle de vie, ainsi que les mécanismes financiers et fiscaux incitant à se former, de telle sorte que les besoins des apprenants soient mieux servis. II faut encourager et évaluer avec rigueur les formes novatrices de cofinancement telles que les comptes formation individuels. Des incitations pourraient être envisagées dans plusieurs domaines : la formation sur le tas, la formation postscolaire à temps partiel, l'alternance travail-études et le recours aux possibilités de formation pour adultes à distance et basée sur l'utilisation des technologies de l'information.

4. Les programmes d'enseignement et les pédagogies doivent privilégier les compétences relationnelles et autres aptitudes non cognitives autant que les facultés cognitives. Les organisations efficaces sont de plus en plus des organisations ap prenantes et novatrices. Du fait de cette évolution, l'aptitude à travailler en équipe, la souplesse et la capacité d'innover et de bien utiliser les connaissances sont de plus en plus demandées (voir OCDE, 2001, chapitre 4).

5. L'investissement dans le capital humain peut également contribuer à développer le capital social. En mettant davantage l'accent sur les aptitudes à résoudre des problèmes en groupe, on pourrait peut-être contribuer à préparer les élèves/étudiants à faire preuve d'une plus grande coopération. On pourrait également utiliser les établissements d'enseignement pour favoriser les atouts des collectivités locales en profitant du fait que d'importantes fractions de la collectivité ont des liens avec ces établissements. Les activités d'intérêt collectif pourraient se dérouler dans les locaux scolaires aux moments de l'année ou de la journée auxquels ils ne sont pas affectés à leur utilisation normale. [Le scénario 3, dans le chapitre 5 de I'Analyse des politiques d'éducation (OCDE, 2001) donne une description 
du rôle que les établissements scolaires pourraient jouer dans ce contexte.]

6. Les dispositifs de formation doivent être ciblés sur les personnes les plus menacées d'être exclues du marché du travail. En règle générale, ceux qui ont le moins de chances de participer à des activités de formation sont ceux qui en ont le plus grand besoin. Dans de nombreux pays de l'OCDE, les inégalités croissantes de revenu observées ces dernières années pourraient être encore accentuées au fur et à mesure que de nouvelles formes d'apprentissage et de nouvelles technologies se dévelop peront. Pour résoudre ces problèmes, il sera nécessaire de modifier les moyens d'action afin par exemple de s'assurer que la fracture numérique ne crée pas d'inacceptables inégalités d'accès à la formation à distance.

L'accès au capital social est en général bénéfique...

... mais le rôle des pouvoirs publics dans le développement du capital social est moins évident que dans le cas du capital humain...

\subsection{Politiques en faveur du capital social}

Les données passées en revue dans ce domaine montrent que le capital social joue un rôle important dans le bien-être, la santé et la recherche d'un emploi et laissent dans une certaine mesure entrevoir la contribution qu'il peut ap porter à la croissance économique. Cela dit, certaines formes de capital social ne sont pas souhaitables - notamment celles qui sont en jeu lorsque des personnes coopèrent à des fins antisociales. Les politiques doivent tenir compte des diverses formes de capital social et du fait qu'elles sont plus ou moins désirables.

Comme on l'a signalé plus haut, le rôle que jouent les pouvoirs publics pour assurer la pérennité du capital social est moins clair que dans le cas du capital humain. Souvent, ce rôle sera peut-être plutôt celui d'un accompagnateur que d'un acteur principal. Parallèlement, les pouvoirs publics ainsi que d'autres instances publiques exercent une influence diffuse, mais puissante si on la considère globalement, sur la formation du capital social. Les organismes à même d'agir sur le capital social sont disséminés dans l'ensemble des secteurs public, privé et associatif. Les niveaux locaux et régionaux d'administration jouent souvent un rôle particulièrement important à cet égard.

Dans l'optique des politiques de lutte contre l'exclusion sociale, il faut surtout admettre que l'accès au capital social contribue à déterminer les chances dans la vie. Certains types précis de capital social peuvent aussi être particulièrement utiles en matière d'exclusion sociale - on peut citer notamment les «liens d'accointance » forgés ind épendamment des frontières que représentent la classe sociale, la communauté ethnique et le sexe. La réussite des immigrés et des personnes issues de minorités ethniques peut être subord onnée à ces relations sociales qui leur permettent d'adhérer à des réseaux clés d'accès et d'influence dans la société. L'une des actions possible serait de tenter d'améliorer le capital social dans les zones ou au sein des communautés dans lesquelles ce dernier laisse à désirer.

Quelques exemples de politiques exerçant actuellement une influence sur le capital social figurent dans l'encad ré 4.1. Les recherches nouvelles consacrées au capital social révèlent la portée de ces initiatives en démontrant que les relations sociales établies dans un contexte donné peuvent 


\section{Encadré 4.1. Politiques en faveur du capital social : quelques exemples}

Les actions en faveur du capital social ne sont pas nouvelles. On a indiqué ci-dessous quelques exemples de politique dont l'incidence dépend du capital social ou qui ont pour effet de le renforcer:

En Italie, renforcer les collectivités et les familles en faveur des enfants. A Pistoia, en Italie, la municipalité met des lieux de rencontre à la disposition des enfants et les familles, grâce à ces structures d'accueil, n'ont pas besoin de faire garder leurs enfants à temps plein. Les parents, grands-parents et autres membres de la famille peuvent suivre des activités de perfectionnement dans ces locaux, qui servent en outre de lieux de réunion aux adultes ainsi que d'aires de jeu et d'expérimentation aux enfants. Ces structures accueillent également les enfants d'âge scolaire ap rès l'école et offrent un centre de documentation et d'information aux enseignants des scuole dell'infanzia et des écoles élémentaires de la ville.

En Irlande du N ord, construire des passerelles grâceà la participation de la collectivité. Le U Ister People's College, organisme bénévole situé à Belfast, propose des programmes de formation structurés, de divers niveaux, à la population locale qui participe à des activités bénévoles et à des travaux d'intérêt collectif. Les formations aux tâches d'encadrement proposées par le College aident les membres de la collectivité à devenir plus efficaces dans leur travail et, par ailleurs, le College a constitué également à Belfast un réseau très efficace à la disposition des travailleurs bénévoles et des mouvements associatifs. Cet établissement a expressément pour mission, entre autres, d'intégrer des représentants à la fois des mouvements nationalistes et unionistes et de contribuer à la paix et à la réconciliation en Irlande du Nord.

$A u$ Danemark, innover à travers la constitution de réseaux. Le Danemark met en œuvre un certain nombre de programmes dont l'objectif est de stimuler l'innovation en créant des relations entre les centres de recherche et le secteur privé. Les partenariats publics-p rivés dans le domaine de la recherche ont été encouragés par le vote de nouveaux textes de loi en matière d'invention et l'adoption de nouvelles réglementations applicables à la participation financière du secteur privé dans les organismes de recherche publics. L'innovation, les partenariats et la constitution de réseaux ont également été favorisés au moyen de «contrats centralisés » en vertu desquels les entreprises, les établissements de services technologiques et les chercheurs travaillent de concert sur des projets de $R \& D$ à vocation commerciale et financés conjointement par les pouvoirs publics et le secteur privé.

être utilisées à des fins extrêmement diverses. Autrement dit, on peut considérer que la participation à des activités sportives, par exemple, procure des avantages allant bien au-delà de la santé physique et du plaisir lié à l'activité elle-même.

Les recherches dans le domaine du capital social se trouvent à un stade précoce et ne permettent pas encore de savoir avec certitude si tel ou tel programme ou telle ou telle politique réussira ou non à atteindre les objectifs qui sont les siens dans ce domaine. Parallèlement, l'élaboration de politiques et leur évaluation offrent le seul moyen d'affiner notre compréhension du capital social et de son utilité. II faut donc considérer les options présentées ci-dessous plutôt comme des id ées méritant d'être creusées, expérimentées et évaluées que comme des programmes bénéficiant déjà d'un effort considérable de recherche.

1. Soutien en faveur des familles. Parmi les moyens d'action, on peut notamment citer l'octroi d'une aide fiscale, l'assouplissement des heures de travail et l'adoption de dispositions visant à encourager les parents à s'impliquer davantage dans la vie de leurs enfants, ou à faciliter cette démarche. II pourrait être utile de moduler le temps de travail pour donner plus de temps libre aux parents ou encore d'en améliorer l'organisation et l'aménagement - étant entendu que ces politiques favorables à la famille ont pour effet à la fois d'aider
... quant à la recherche sur le capital social, elle se trouve à un stade précoce.
Quelques idées sont susceptibles de faire l'objet de projets pilotes et d'évaluations. 
les parents en activité et d'encourager un plus grand nombre d'entre eux, et en particulier les femmes, à accéder au marché du travail s'ils le souhaitent.

2. Soutien en faveur du bénévolat. Les possibilités d'action englobent à la fois les mesures prises du côté de la demande pour encourager le financement d'organisations qui ont effectivement recours à des bénévoles et les mesures prises du côté de l'offre qui incitent les employeurs à octroyer à leurs salariés du temps libre pour mener des activités associatives.

3. Les processus de décision des pouvoirs publics. Les systèmes démocratiques qui font intervenir la population constituent non seulement un moyen de prendre de meilleures décisions, mais également un élément essentiel de l'ensemble du tissu social. La participation des citoyens à l'exercice du pouvoir et le fait que l'administration publique soit proche de la population peuvent contribuer à dynamiser les collectivités locales et à mobiliser les énergies au profit d'une action sociale constructive.

4. TIC et capital social. Les nouvelles formes de TIC ainsi que les moyens de communication plus traditionnels peuvent être utilisés pour aider à établir un lien entre la population et son voisinage ainsi qu'avec des communautés plus éloignées. Les TIC offrent aux pouvoirs publics de nouvelles possibilités pour consulter les citoyens, communiquer avec eux et soumettre à leur examen leurs propres actions. Les réseaux électroniques peuvent également servir à communiquer des renseignements et à faciliter les opérations commerciales, surtout en l'absence d'informations adéquates 3 . Les nouveaux médias créent de plus grandes possibilités de formation informelle, y compris à distance. II importe que les groupes défavorisés aient davantage accès à ces nouveaux médias afin de pouvoir tirer pleinement parti des nouveaux réseaux et autoroutes de l'information. Les environnement informels d'ap prentissage gagnent de plus en plus en importance à mesure que les pays s'orientent vers des types de formation diversifiés, personnalisés et fondés sur la demande.

5. O rganisation locale des soins de santé. La planification et la prestation des soins de santé à l'échelon local, ce qui permet aux personnes âgées et aux autres groupes de population concernés de rester à proximité de leurs familles et dans leur environnement habituel, peuvent constituer un autre moyen de maintenir les liens sociaux tout en en retirant des avantages sur le plan de la santé, qui, selon les recherches, sont liés au capital social4.

\subsection{Les lacunes à combler et les recherches à mener à l'avenir}

Dans ce rapport, un très grand nombre de thèmes ont été abordés et de multiples pistes de recherche pour l'avenir ont été repérées. Toute liste de projet sera inévitablement très sélective. Des lacunes dans nos connaissances et notre compréhension s'observent, semble-t-il, essentiellement dans les domaines suivants : 
1. Élargir la mesure du capital humain, en particulier la mesure directe des compétences, qui ne se limitent pas au « savoir lire-écrirecompter » mais englobent aussi, entre autres, l'aptitude à travailler en équipe, à résoud re des problèmes et à utiliser les TIC. S'il est vrai que ces compétences, ou certains de leurs aspects, peuvent dépendre du contexte culturel, il importera malgré tout d'en améliorer la mesure même lorsque, par exemple, les instruments utilisés dans les enquêtes internationales sont inappropriés. II s'agira notamment de continuer à exploiter les résultats de l'Enquête internationale sur la littératie des adules (IALS) afin de couvrir une palette plus large de compétences utiles dans la vie courante.

2. Arriver à comprend re plus clairement comment la demand e de capital humain évolue et continuera d'évoluer tant quantitativement que qualitativement. Ces travaux seront primordiaux pour orienter les projets actuellement entrep ris à la fois pour former les travailleurs à la nouvelle économie du savoir et pour résoudre les problèmes auxquels sont confrontés ceux dont les compétences sont limitées ou dépassées. Des travaux complémentaires pourraient être nécessaires afin d'intégrer, dans la réflexion au sujet de l'évolution de la demande de compétences, d'un côté la question de la structure des programmes d'enseignement scolaire, et de l'autre celle de l'équilibre à trouver entre la formation initiale et l'éducation et la formation tout au long de la vie.

3. Préciser les liens entre le capital humain et le capital social afin d'étudier comment les réseaux sociaux peuvent favoriser l'instruction des individus et comment l'instruction peut promouvoir le capital social.

4. Approfondir la définition conceptuelle de la notion de capital social, de préférence en rattachant cet exercice à des travaux empiriques. A cette fin, il faudra également affiner notre classification des différentes formes de capital social, déterminer les domaines d'analyse et les types de capital social pour lesquels I'utilisation de cette notion est la plus fructueuse.

5. Améliorer la mesure du capital social au sein de groupes sociaux de même que celle de l'accès individuel à ce capital, ces deux types de mesures devant être distincts mais articulés entre eux. Un certain nombre de questionnaires ont été élaborés à cette fin. Les mesures utilisées dans Putnam (2000a) établissent un lien avec les comportements ou les attitudes recensés à l'occasion d'enquêtes effectuées auprès des personnes. Les dispositions déclarées à l'égard de la confiance ou les activités concernant la socialisation informelle, le comportement des électeurs, l'adhésion à divers types d'organisations et le bénévolat dans des projets associatifs constituent des indicateurs importants des normes et des modes de comportement en réseau de la population dans son ensemble.

6. Grâce à l'évaluation des actions menées, arriver à mieux comprendre celles qui permettent avec succès de promouvoir le capital social ainsi que les contextes dans lesquels le développement du
Il serait utile de poursuivre les travaux sur la mesure du capital humain, sur l'évolution de la demande et sur la nature des liens entre le capital humain et le capital social.

Des recherches plus poussées pourraient permettre d'expliciter la notion de capital social et d'en améliorer la mesure.

Nous devons également déterminer les actions efficaces. 
capital social procure le maximum d'avantages. On a donné plus haut une idée des domaines se prêtant à une évaluation.

Le nouveau débat sur le rôle du capital social pourrait ressembler à celui consacré au capital humain auquel I'OCDE a largement contribué dans les années 60.

L'OCDE pourrait encore apporter sa contribution.

\subsection{Conclusion}

Dans les années 60, I'OCDE a largement contribué à la définition et à la reconnaissance de la notion de capital humain dans l'activité économique. Une génération plus tard, alors qu'il subsiste d'importantes ambiguïtés conceptuelles, des incertitudes dans les conclusions des recherches et de sérieux problèmes de mesure, c'est le capital social qui est à l'ord re du jour. Tout en passant en revue les tout derniers éléments d'information au sujet du capital humain, on a tenté ici de faire avancer notre compréhension du capital social.

Dans le monde entier, des travaux très divers de recherche, $d$ 'analyse et d'élaboration de politiques sont en cours au sujet du capital humain et, de plus en plus, du capital social. Afin de pouvoir diffuser des conclusions fond amentales, il est extrêmement important de suivre l'évolution de l'ensemble de ces travaux et de mettre en commun les données d'expérience recueillies, qu'il s'agisse des conséquences des actions menées ou de problèmes de mesures. La mission de I'OCDE sera de repérer les domaines où sa propre contribution peut apporter une valeur ajoutée. Trois domaines pourraient être retenus à cette fin :

- L'OCDE a joué un rôle important en regroupant des indicateurs, comparables à l'échelle internationale, de la formation du capital humain et en réal isant sur la base de ces indicateurs une analyse des actions menées. Elle peut poursuivre ces travaux et les ap profondir en ajoutant l'analyse de la complémentarité possible du capital humain et du capital social.

- Le capital social suscite un intérêt grandissant chez les décideurs et responsables politiques, mais rares sont les exemples qui montrent clairement que les résultats des recherches sur le capital social ont été mis en application sans réserve. Cette notion reste vague à de nombreux égards et il n'est pas rare que la terminologie varie. De plus, l'idée de capital social est ap plicable à une grande diversité de contextes et, pour l'instant, on ne sait pas encore au juste où son utilisation se révélera la plus utile. Par ailleurs, l'efficacité des différentes politiques destinées à favoriser le capital social constitue pour l'instant un domaine de recherche quasiment inexploré. Ces considérations donnent à penser que I'OCDE pourrait peut-être utilement organiser un séminaire à haut niveau, dont l'objet serait de mettre en commun les données d'expérience et les idées théoriques des pays Membres à la lumière des nouveaux résultats de la recherche sur le capital social.

- Il est essentiel d'améliorer la mesure du capital social, et des travaux sont en cours dans un certain nombre de pays pour mettre au point des instruments d'enquête. L'une des options serait que I'OCDE envisage la possibilité d'une centralisation internationale des ressources dans ce domaine - peut-être grâce à l'élaboration d'un questionnaire commun, lié aux travaux que I'Organisation consacre en 
permanence aux indicateurs de l'enseignement. L'une des solutions méritant d'être approfondies serait, par exemple, d'associer la mesure du capital humain et du capital social dans une enquête sur les compétences des adultes. Ce moyen permettrait, en principe, d'évaluer l'incidence conjointe et respective de ces deux formes de capital sur les chances dans la vie.

Une tâche primordiale incombant aux pays de l'OCDE est d'assurer une croissance économique continue et d'améliorer le bien-être de tous. Dans une économie globale en mutation rapide, l'utilité des compétences sociales et humaines pour accomplir cette tâche est plus importante que jamais. Notre mission sera de parvenir à mieux comprendre le rôle de ces compétences, autrement dit le capital humain et social. Grâce à cela, nous serons à même de mieux utiliser les aptitudes humaines et de concevoir de nouvelles façons de travailler ensemble pour accroître le bien-être de tous - et nous laisserons ainsi aux générations futures au moins autant de chances que nous en avons eues.
L'attention portée au capital humain et au capital social contribuera à préserver le bien-être des générations futures. 


\section{Notes}

1. Voir OCDE (2001, chapitre 4), où est examiné le problème de la définition des nouvelles qualifications requises par l'économie du savoir.

2. Epstein (1995) donne une idée de la façon dont pourrait fonctionner un partenariat entre les écoles, les familles et les collectivités : l'école fonctionnerait plus comme une famille en accueillant non seulement les enfants mais aussi leurs parents, et la famille fonctionnerait plus comme une école en encourageant les enfants à apprendre.

3. Ces réseaux électroniques pourraient comprendre, par exemple, des bulletins d'information servant à communiquer des offres d'emploi, des offres de bénévolat, etc., dans une zone précise.

4. Voir dans la section 3.7 plus haut les données passées en revue au sujet de l'incidence des liens sociaux et de la qualité des relations personnelles. 


\section{Annexe A}

\section{QUELQUES INDICATEURS DU BIEN-ÊTRE}

Les enquêtes sur l'opinion du public et la qualité de la vie, menées dans divers pays de l'OCDE, notamment la World Values Survey, tendent à démontrer que globalement le bien-être progresse plus lentement que le PIB. II s'ensuit que dans le monde développé, la croissance économique est peut-être entrée dans une phase de rendements décroissants (Inglehart, 2000 ; Eckersley, 1998). L'une des conséquences est qu'il faut accorder une plus grande attention à la qual ité de la croissance économique en tenant compte d'autres changements survenant éventuellement dans l'environnement physique ou social, qui sont liés à cette croissance et qui influent sur le bien-être.

Le PIB et d'autres indicateurs fondés sur la comptabilité nationale (PIB « vert », «épargne véritable », etc.) sont calculés à partir des estimations monétaires des flux de revenu et des ajustements relatifs aux stocks mesurables. La valeur des variations de stocks ou le prix d'une activité donnée sont déterminés sur la base de ce que les consommateurs et la société sont prêts à payer. Les coûts et avantages hors marché ne peuvent être aisément intégrés dans ce système.

Cette annexe présente, à titre d'exemple, certains indicateurs qui ont été utilisés pour effectuer des comparaison internationales ou intertemporelles de certains aspects du bien-être. Chaque exemple représente des aspects du bien-être dont il n'est pas tenu compte dans les indicateurs fondés sur le PIB ou dans des indicateurs analogues. Il est possible de donner une idée de l'ampleur probable des ajustements apportés au PIB ou aux investissements en se reportant à certaines des étud es examinées plus loin. Utilisant les données concernant les États-Unis en 1965, Nord haus et Tobin (1972) constatent que la valeur des loisirs et d'autres activités, dont il n'est pas tenu compte dans le PIB, correspondent environ à 100 et 50 pour cent respectivement du PIB des États-Unis. Jorgenson et Fraumeni (1987), se basant sur les données relatives auxÉtats-Unis pour 1982, chiffrent l'investissement dans le capital humain, selon des estimations, à plus de 50 pour cent de la totalité du produit intérieur brut privé.

Certes, nombre des méthodes utilisées pour mesurer le bien-être sont relativement nouvelles, mais les raisons qui les justifient ne le sont pas. Nordhaus et Tobin (1972) ont proposé un indicateur composite du bien-être économique net, intégrant des éléments nouveaux dans un indicateur plus vaste de la consommation et du bien-être durables. Ils ont inclus dans le PIB :

- des estimations de la valeur de certaines activités telles que la garde des enfants par leurs parents ainsi que d'autres services domestiques et les loisirs1;

- les investissements matériels des ménages et des administrations publiques (les biens de consommation durables, par exemple, dans le cas des ménages).

Les éléments exclus du PIB sont:

- les dépenses intermédiaires consacrées à des « activités qui de toute évidence ne sont pas des sources directes de bien-être, mais sont malheureusement nécessaires à d'autres activités pouvant quant à elles générer du bien-être » (op. cit., p. 7) (il s'agit par exemple du coût des trajets domicile-travail, des dépenses publiques imputées à la police, à l'assainissement, à la voirie, à la défense nationale) ;

- I'amortissement du capital ainsi que les dépenses d'investissement requises pour satisfaire les impératifs de croissance à l'avenir² ; 
- «les désagréments de la vie urbaine »3 (par exemple, le coût de la pollution, des petits déchets, des encombrements et du bruit) ;

- l'éducation et la santé (du fait qu'il s'agit de biens intermédiaires dont les retombées sont déjà factorisées dans l'accroissement du revenu).

A la différence de Nord haus et de Tobin, Jorgenson et Fraumeni (1987) ont inclus dans leur mesure du bien-être les investissements dans l'éducation. Tout comme eux, cependant, ils ont pratiqué des ajustements au titre des subventions, des dépenses d'équipement et de consommation des ménages et de la production de loisirs par les ménages. La valeur des investissements dans l'éducation correspond, dans les estimations, à la somme de la valeur actuelle des revenus de toute une vie pour l'ensemble des individus et de la valeur imputée des revenus auxquels ont renoncé ceux qui poursuivent des études4. Aucune déduction n'est faite pour tenir compte du coût que représente l'éducation ou l'instruction des enfants. Kendrick (1976), en revanche, inclut des estimations du coût de l'éducation, des coûts de formation supportés par les employeurs, des dépenses de formation informelle (dans les bibliothèques, les musées ou au titre des informations diffusées par les milieux de la presse et de l'édition) et de la valeur des dépenses de recherche et développement.

Les indicateurs utilisés sont dans la plupart des cas composites et partiellement non monétaires. D'autres font intervenir des estimations, calculées sur une base monétaire, de diverses évolutions ou résultats observés dans le domaine social et supposent leur intégration dans un système de comptabilité nationale, soit directement, soit par l'intermédiaire de comptes satellites. Parmi les indices synthétiques du bien-être utilisés, peuvent par exemple être cités :

- L'indicateur de progrès réel (IPR), qui est un indicateur du bien-être, calculé sur une base monétaire à partir de données remontant jusqu'en 19505. Le cadre de comptabilisation classique est élargi afin d'inclure, en plus de la production économique conventionnelle, les contributions économiques des familles, des communautés et de I'habitat naturel.

- L'indicateur du développement humain (IDH) élaboré par les Nations Unies mesure les résultats globaux enregistrés par un pays pour trois aspects fondamentaux de ce développement, à savoir la longévité, le savoir et les ressources économiques. II mesure plus précisément l'espérance de vie, le niveau d'instruction (taux de « littératie » - maîtrise des savoirs fondamentaux des adultes et taux de scolarisation dans le primaire, le secondaire et le supérieur considérés ensemble) et le niveau de revenu par habitant pondéré des parités de pouvoir d'achat (Programme des Nations Unies pour le Développement, 1990, 2000).

- Un indice de santé sociale (ISS) a été mis au point au Canada à partir de l'indice de santé sociale de Fordham6. II s'agit d'un indice créé sur la base d'une série d'indicateurs socio-économiques, qui mesure les progrès d'un pays donné par rapport à ses performances passées 7 .

- L'indice de bien-être économique durable (IBEED) mesure diverses activités qui contribuent à la qualité de la vie8. La pollution de l'air occasionnée par l'activité économique est soustraite et la valeur du travail domestique non rémunéré est incluse. Cet indice couvre également des aspects tels que les inégalités de revenus, divers préjudices environnementaux et l'appauvrissement du patrimoine naturel. A ce jour, I'IBEED a été calculé pour le Royaume-Uni, I'Allemagne, l'Autriche, la Suède, les Pays-Bas, I'Italie et les États-Unis.

L'indice du bien-être économique a été élaboré par Osberg (1985), puis explicité et illustré par Osberg (2001) à I'aide de données concernant les États-Unis, le Royaume-Uni, le Canada, l'Australie, la Norvège et la Suède sur la période 1980-19969. Osberg pense que les éléments non pris en considération pour la mesure du PIB peuvent être particulièrement pertinents du point de vue du capital social. Son indice du bien-être économique s'articule autour de quatre composantes:

- Les flux de consommation effectifs par habitant, qui incluent la consommation de biens et services marchands ou non marchands, l'évolution de l'espérance de vie et des loisirs (consommation marchande par habitant, dépenses publiques par habitant, variation de la durée du travail). 
- L'accumulation nette par la société de réserves de ressources productives, y comp ris l'accumulation nette de capital physique et d'actifs immobiliers par habitant, l'accumulation nette de capital humain (dépenses annuelles d'éducation rapportées à la population adulte totale) et la recherche-développement par habitant, moins la variation nette de l'endettement extérieur et le coût social de la dégradation de l'environnement.

- La distribution du revenu, mesurée par le coefficient d'inégalité de Gini (inégalités ap rès impôts), ainsi que le degré et l'incidence de la pauvreté.

- L'insécurité économique liée au chômage, aux problèmes de santé, à la pauvreté chez les parents isolés et les personnes âgées.

Les éléments de l'indice sont présentés dans le graphique A.1. On peut utiliser différentes pondérations, calculées à partir des valeurs et des jugements des utilisateurs, pour associer les quatre principaux éléments qui sont

\section{Graphique A.1. Illustration de l'indice d'Osberg}

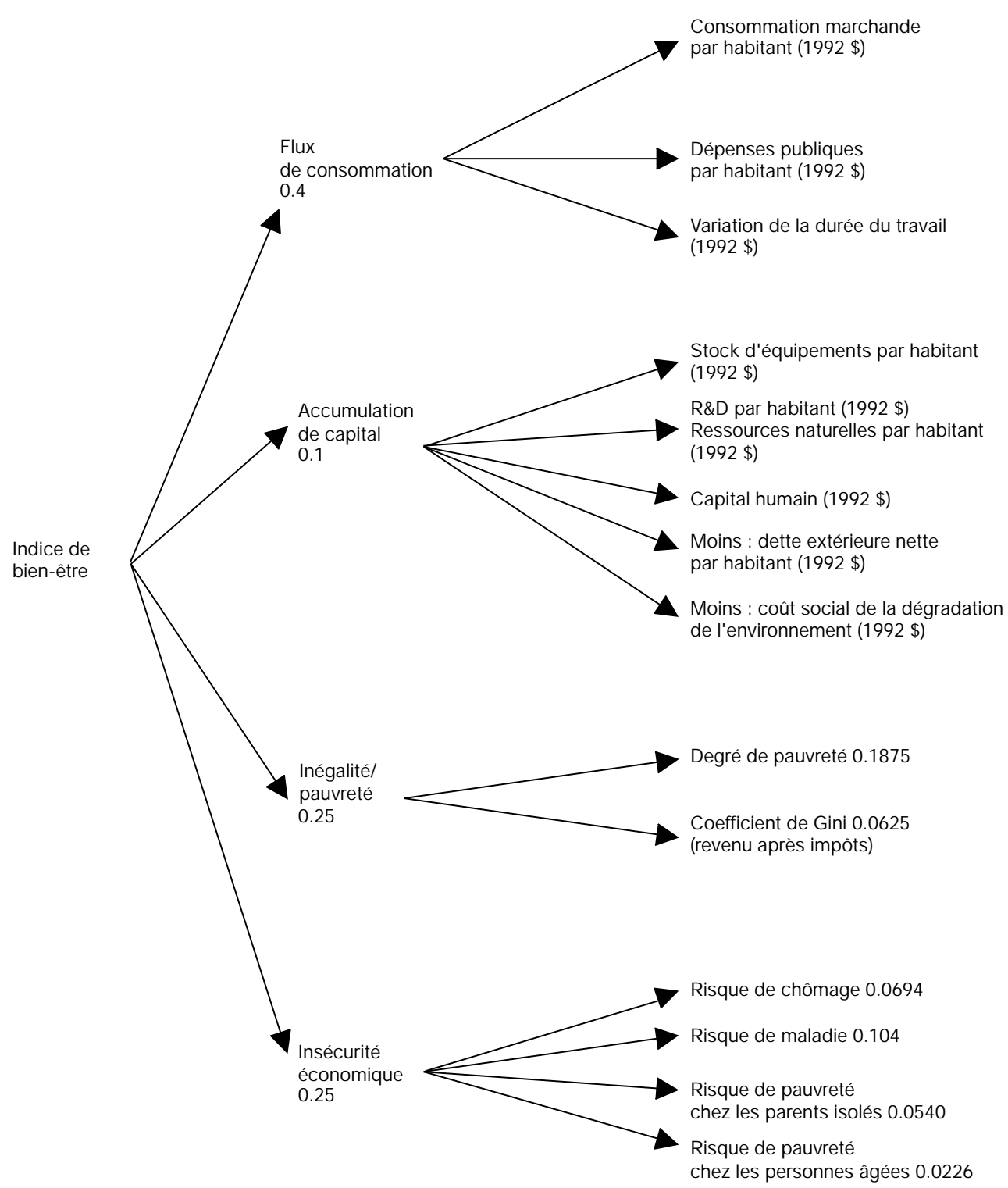

Source: Osberg (2001). 
la consommation moyenne, les situations héritées de génération en génération, l'inégalité/la pauvreté et l'insécurité. Un aspect essentiel dans cet indice est d'attribuer une pondération explicite aux questions de distribution, qu'il s'agisse des affectations à la consommation présente et future ou de la répartition des revenus et des possibilités économiques entre les différents sous-groupes durant la période considérée. Dans la mesure où les quatre principaux éléments sont signalés de façon distincte, il est aisé d'analyser l'influence qu'a la variation des pondérations sur les tendances globales observées. Les figures du graphique A.2 présentent les résultats obtenus avec les deux pondérations (standard et variante) pour cinq pays : I'Australie, le Canada, les États-Unis, le Royaume-Uni et la Suède.

Ces résultats montrent que dans chaque pays considéré, et bien qu'à des degrés variables, la croissance du PIB par habitant dépasse la croissance du bien-être économique. Dans tous les pays, l'évolution négative de l'inégalité/pauvreté et des indicateurs d'insécurité se traduisent par un retard de la progression du bien-être économique par rap port à l'accroissement du PIB. Aux États-Unis, le PIB par habitant a augmenté approximativement de 30 pour cent au cours de la période allant de 1980 à 1997, mais l'indice de bien-être économique est resté presque constant, augmentant seulement de 4 pour cent au cours de cette période. Au Royaume-Uni, le PIB par habitant a augmenté encore plus ( 40 pour cent), mais l'indice de bien-être économique a fléchi de près de 10 pour cent. Osberg note que ces résultats témoignent de la nette progression des inégal ités économiques au cours de cette période. Dans le cas des États-Unis, un facteur supplémentaire est l'augmentation importante des heures de travail. Selon Osberg, le recul de cet indice au Royaume-Uni et en Suède est sensible à la pondération relative de la consommation courante par rapport à la répartition des revenus et à l'insécurité. Dans le cas de l'Australie et du Canada, dont la dépendance à l'égard de la production de matières premières est plus grande, le PIB par habitant fluctue davantage que l'indice de bien-être économique. S'agissant du Canada, I'utilisation d'autres pondérations possibles pour l'insécurité économique et les inégalités ne modifie pas sensiblement la valeur de l'indice de bien-être économique.

Vouloir résumer le bien-être de sociétés complexes et de plus en plus diverses «implique nécessairement de formuler une série de jugements éthiques et statistiques »(Osberg, 2001). Les différentes approches présentées plus haut sont des tentatives importantes faites pour élaborer des indicateurs plus précis, mais toutes, à des degrés divers, sont limitées par:

- Le choix des indicateurs ou des composantes parmi tous les aspects qu'il faudrait prendre en compte pour avoir un indice complet.

- La pondération des différents indicateurs et composantes.

- L'interprétation de l'évolution quantitative de ces composantes par rapport aux tendances du PIB, alors que ces composantes sont moins précises (comme c'est le cas pour les ind icateurs du chômage).

Additionner divers indicateurs puis effectuer une pondération et une modulation arbitraires peuvent être source de divergences sans fin sur la pondération des composantes du bien-être. Les lecteurs décideront par eux-mêmes si l'une des approches proposées est, malgré ses imperfections, préférable à la mesure par le PIB. II importe de déterminer s'il ne vaut pas mieux disposer d'une mesure partielle que de n'avoir aucune mesure synthétique des autres aspects du bien-être. 


\section{Graphique A.2. Comparaisons internationales des tendances du bien-être économique}

_ Indice du PIB par habitant . - Indice du bien-être, pondération standard - - Indice du bien-être, variante

1.50

1.40

1.30

1.20

1.10

1.00

0.90

0.80

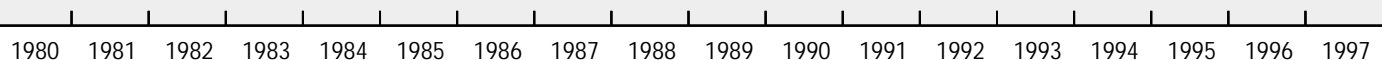

— Indice du PIB par habitant

..- Indice du bien-être, pondération standard

- - Indice du bien-être, variante

1.50

\section{Canada}

1.40

1.20

1.10

1.00

0.90

0.80

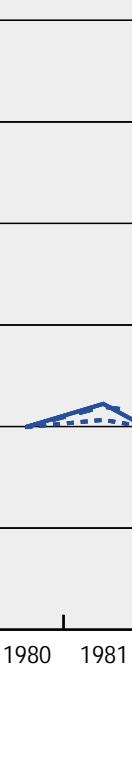

1.50

1.40

1.30

1.20

1.10

1.00

0.90

Source : Osberg (2001), partie A. 
Graphique A.2. Comparaisons internationales des tendances du bien-être économique (suite)

— Indice du PIB par habitant - - - Indice du bien-être, pondération standard - - Indice du bien-être, variante

1.50

\section{Suède}

1.40

1.30

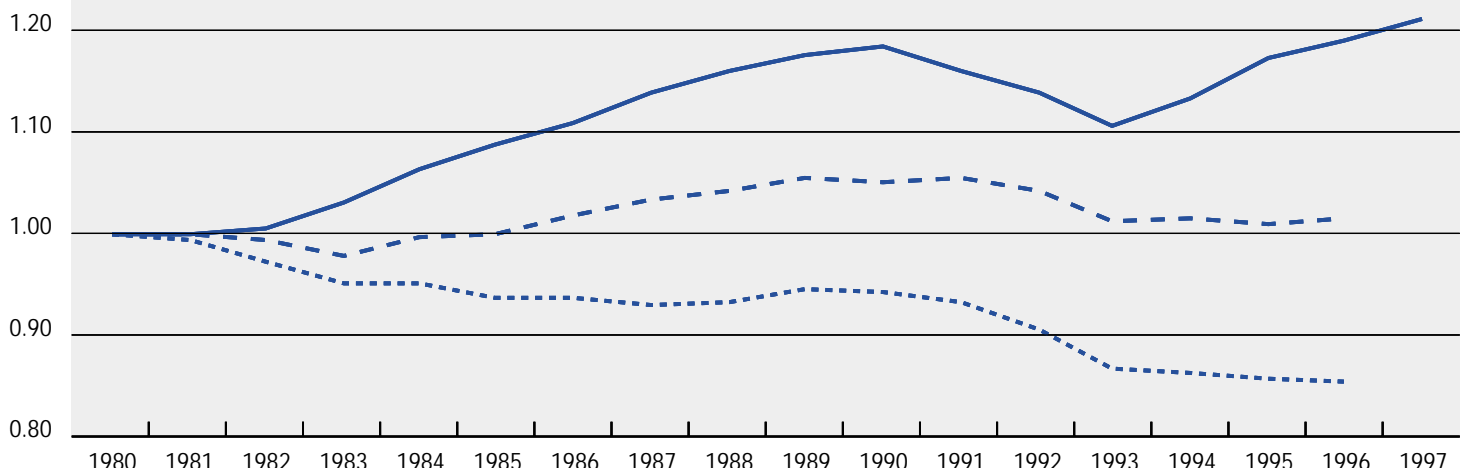

_ Indice du PIB par habitant - - - Indice du bien-être, pondération standard - - Indice du bien-être, variante

1.50

\section{Royaume-Uni}

1.30

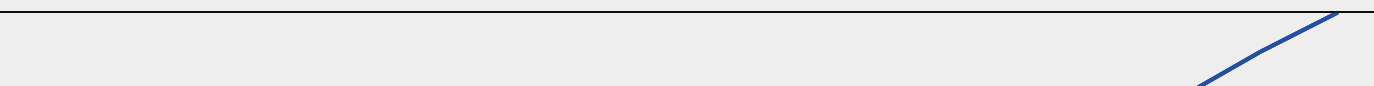

1.20
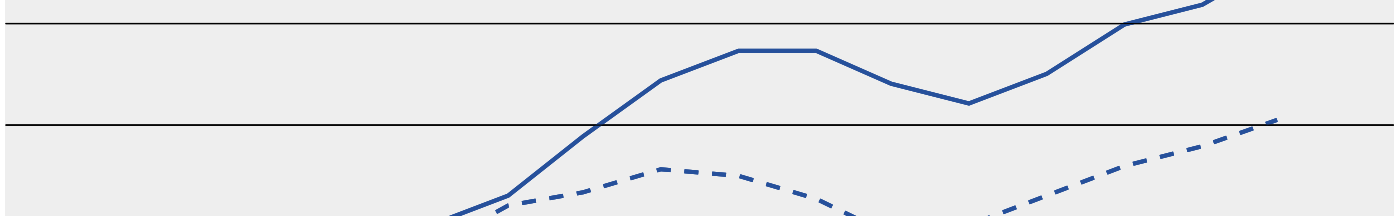

1.10

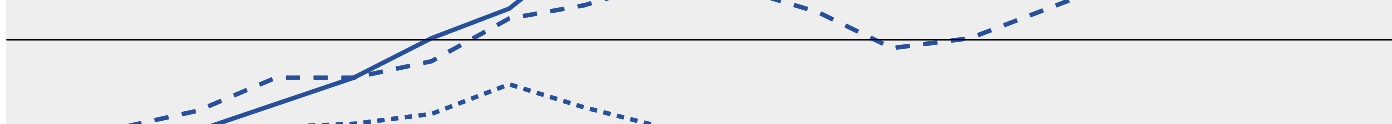

1.00
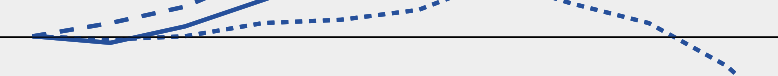

0.90

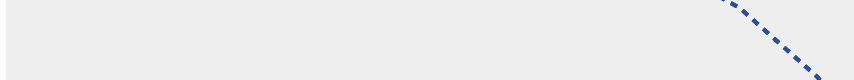

0.80

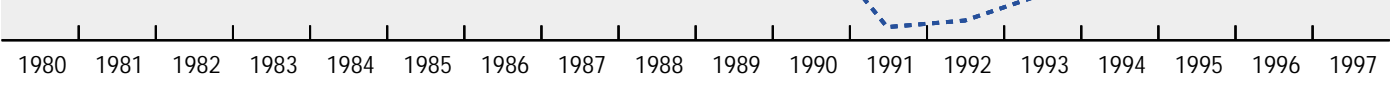

Source : Osberg (2001), partie A. 
Graphique A.2. Comparaisons internationales des tendances du bien-être économique (suite)

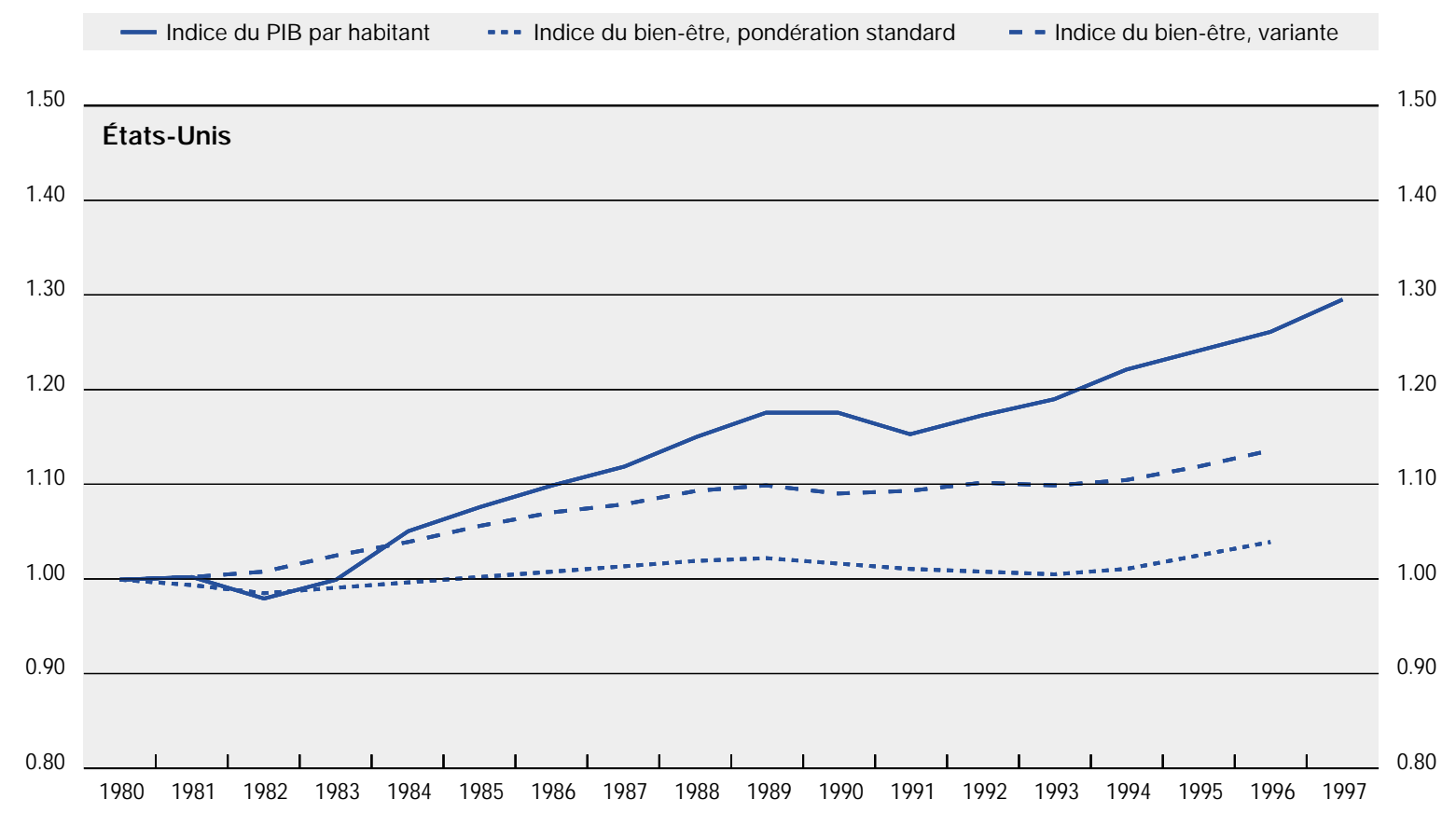

Source : Osberg (2001), partie A. 


\section{Notes}

1. Pour estimer la valeur des loisirs, on a multiplié le nombre estimé d'heures de loisir de la population âgée de 14 ans et plus par les taux de salaire (ce qui correspond au coût d'opportunité des loisirs).

2. Est exclue de la mesure du bien-être économique, la fraction des dépenses d'investissement requise pour maintenir un ratio capital-production constant, la consommation progressant à un rythme compatible avec celui de l'accroissement démographique et du progrès technologique.

3. Faute de données, aucun ajustement n'est introduit au titre de la dégradation de l'environnement.

4. La valeur imputée de la perte de revenu de ceux qui poursuivent des études est égale à l'écart entre les revenus p révisonnels du travail de ceux qui ont fait des études et de ceux qui n'en ont pas fait.

5. L'indice de progrès réel inclut les éléments suivants : consommation personnelle; consommation personnelle corrigée des inégalités de revenu ; valeur du travail domestique et de la garde des enfants par leurs parents ; créances ou emprunts nets vis-à-vis de l'extérieur ; dépenses d'équipement et de consommation des ménages ; services liés aux grandes voies routières et aux rues ; coût du sous-emploi ; valeur du travail bénévole ; dépenses nettes d'investissement ; épuisement de ressources non renouvelables ; coût de l'éclatement des familles ; dégradation durable de l'environnement ; coût de la pollution atmosphérique ; coût des biens de consommation durables ; coût de la pollution de l'eau ; coût des trajets domicile-travail ; coût de la criminalité ; disparition de zones humides; coût du bruit et de la pollution par l'ozone ; coût de la réduction des pollutions domestiques ; diminution des temps de loisirs.

6. Voir également : Index of Social Health (1995), Monitoring the Social Well-Being of the Nation, Ford ham Institute for Innovation in Social Policy, Tarrytown, N.Y.

7. L'indice de santé sociale (Brink et Zeesman, 1997) inclut les éléments suivants : mortalité infantile ; maltraitance des enfants ; pauvreté des enfants ; suicides chez les adolescents ; toxicomanie ; taux élevés d'abandon de la scolarité ; chômage ; revenus hebdomadaires moyens ; pauvreté chez les personnes de 65 ans et plus ; dépenses de santé des personnes de 65 ans et plus non prises en charge par le système; accidents routiers mortels liés à la consommation d'alcool ; homicides; bénéficiaires de l'aide sociale ; fossé entre les riches et les pauvres ; accès à un logement abordable.

8. L'IBEED se compose notamment des éléments suivants : dépenses de consommation ; inégalités de revenu ; services liés au travail domestique et aux biens de consommation durables ; services liés aux rues et grandes voies routières ; dépenses publiques pour la santé et l'éducation ; coût des trajets domicile-travail ; coût de la lutte contre les pollutions domestiques; coût des accidents automobiles ; coûts de la pollution de l'eau et de l'air et des nuisances sonores ; disparition d'habitats naturels ; disparition de terres agricoles ; épuisement de ressources non renouvelables; coûts du changement climatique et de la destruction de l'ozone ; produit intérieur brut (voir le site de Friends of the Earth : http://www.foe.org.uk/campaigns/sustainable_development/progress).

9. Osberg fournit aussi des mesures pour 8 autres pays Membres de I'OCDE, mais les données de base sont parfois moins complètes (cf. Osberg, 2001). 
Annexe B

\section{ÉVOLUTION DE L'ENVIRONNEMENT SOCIAL ET ÉCONOMIQUE - QUELQUES TENDANCES}

\section{Graphique B.1. Produit intérieur brut réel par habitant à prix constant, moyenne de l'OCDE, 1966-99}

20000
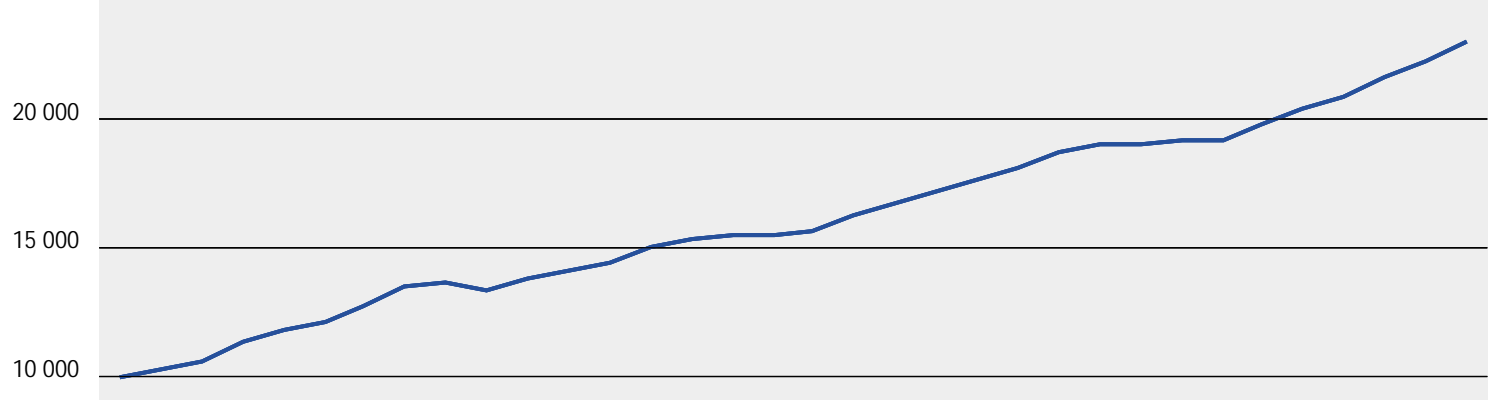

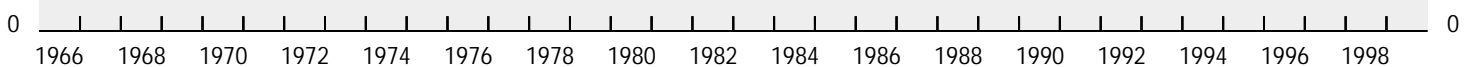

Pays pris en compte : Allemagne, Australie, Autriche, Belgique, Canada, Danemark, Espagne, États-Unis, Finlande, France, Grèce, Irlande, Islande, Italie, J apon, Luxembourg, Norvège, Nouvelle-Zélande, Pays-Bas, Portugal, Royaume-Uni, Suède, Suisse.

Source: OCDE. Produit intérieur brut réel ajusté au prix du dollar de 1998, basé sur les parités de pouvoir d'achat. 
Graphique B.2. Tendances de l'inégalité des revenus pour l'ensemble de la population dans certains pays de l'OCDE, milieu des années 80 et 90

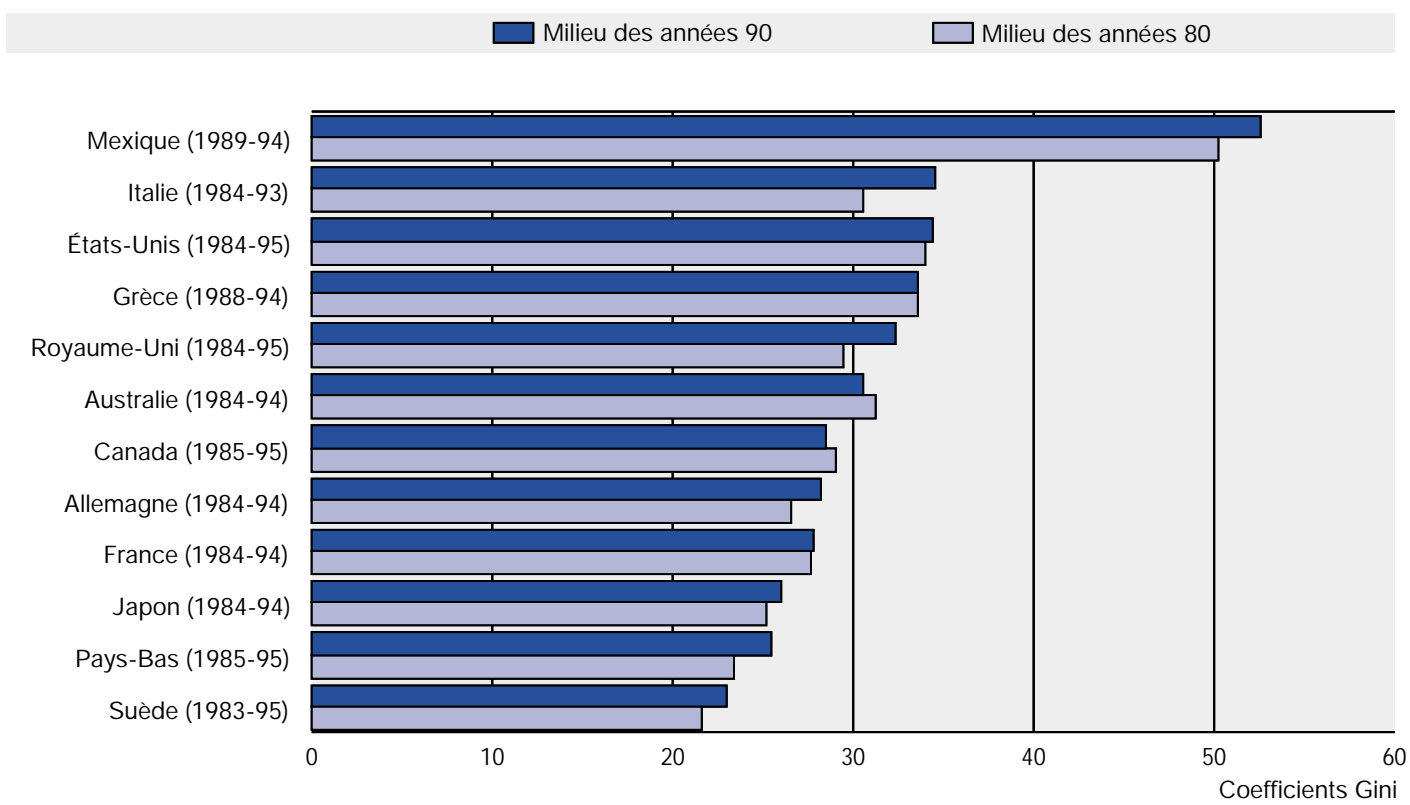

Note: Selon le revenu disponible. Élasticité d'échelle équivalente $=0.5$.

Source : OCDE (1999), «Trends in Income Distribution and Poverty in the OECD Area », document gratuit (en anglais uniquement), tableau 2.2. 


\section{Graphique B.3. Pourcentage d'enfants vivant dans une pauvreté relative dans certains pays de l'OCDE, années 90}

Pourcentage vivant dans des ménages au-dessous de $50 \%$ du revenu médian

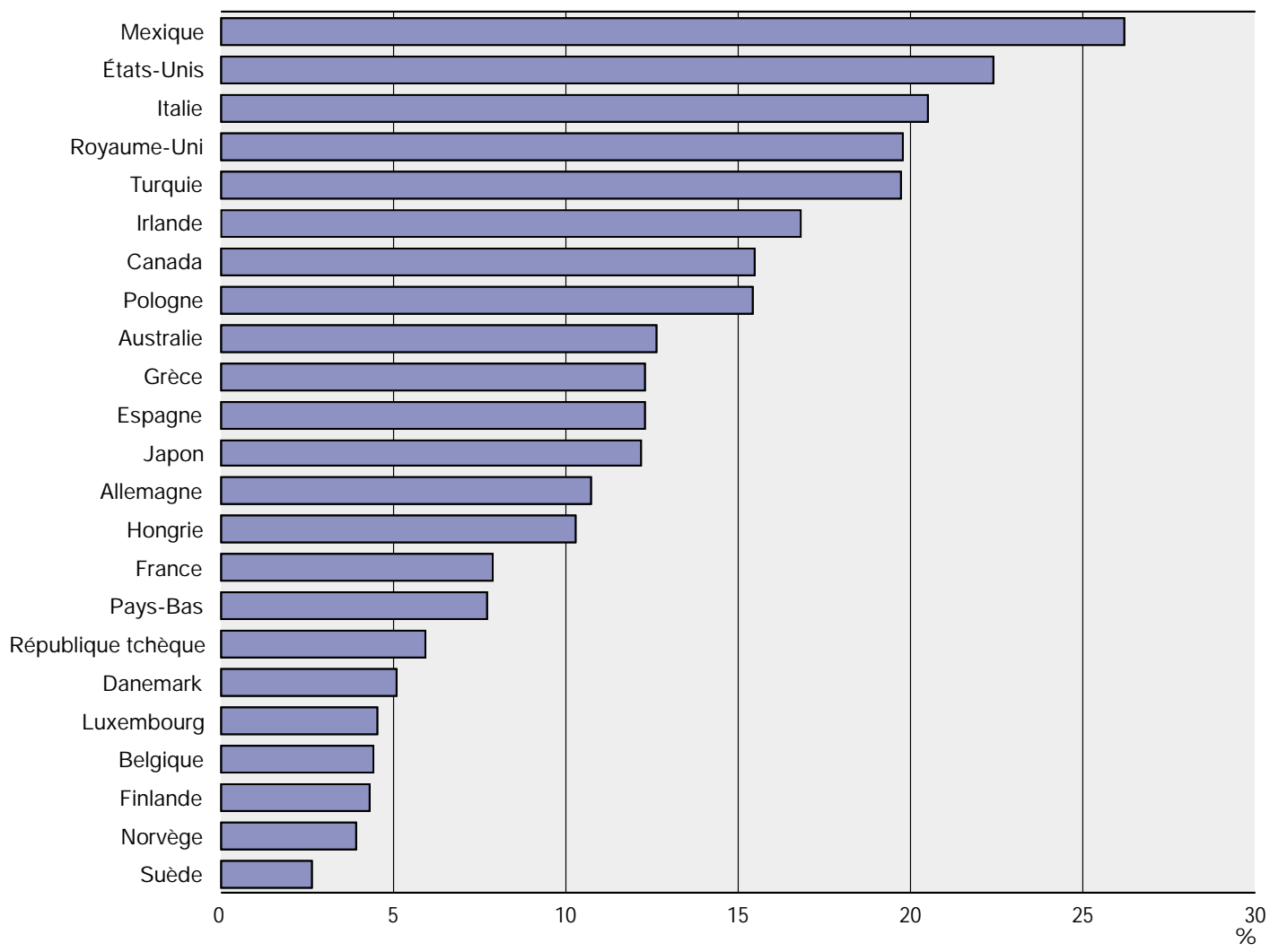

Note: Le pourcentage d'enfants vivant dans une pauvreté relative est défini comme le pourcentage vivant dans des ménages se situant audessous du seuil de $50 \%$ du revenu médian. Le taux de pauvreté porte sur les années suivantes : 1990 (Espagne), 1992 (Belgique, Danemark et J apon), 1994 (Canada, France, Allemagne, Grèce, Hongrie, Luxembourg, Mexique, Pays-Bas, Turquie), 1995 (Finlande, Italie, Norvège, Pologne, Suède, Royaume-Uni), 1996 (République tchèque), 1996-97 (Australie) et 1997 (Irlande, États-Unis).

Source: UNICEF (2000), «A league table of child poverty in rich nations », Innocenti Report Card No. 1, UNICEF Innocenti Research Centre, Florence. 
Graphique B.4. Taux de chômage des 25-54 ans et des 15-24 ans dans certains pays de l'OCDE, 1975-99

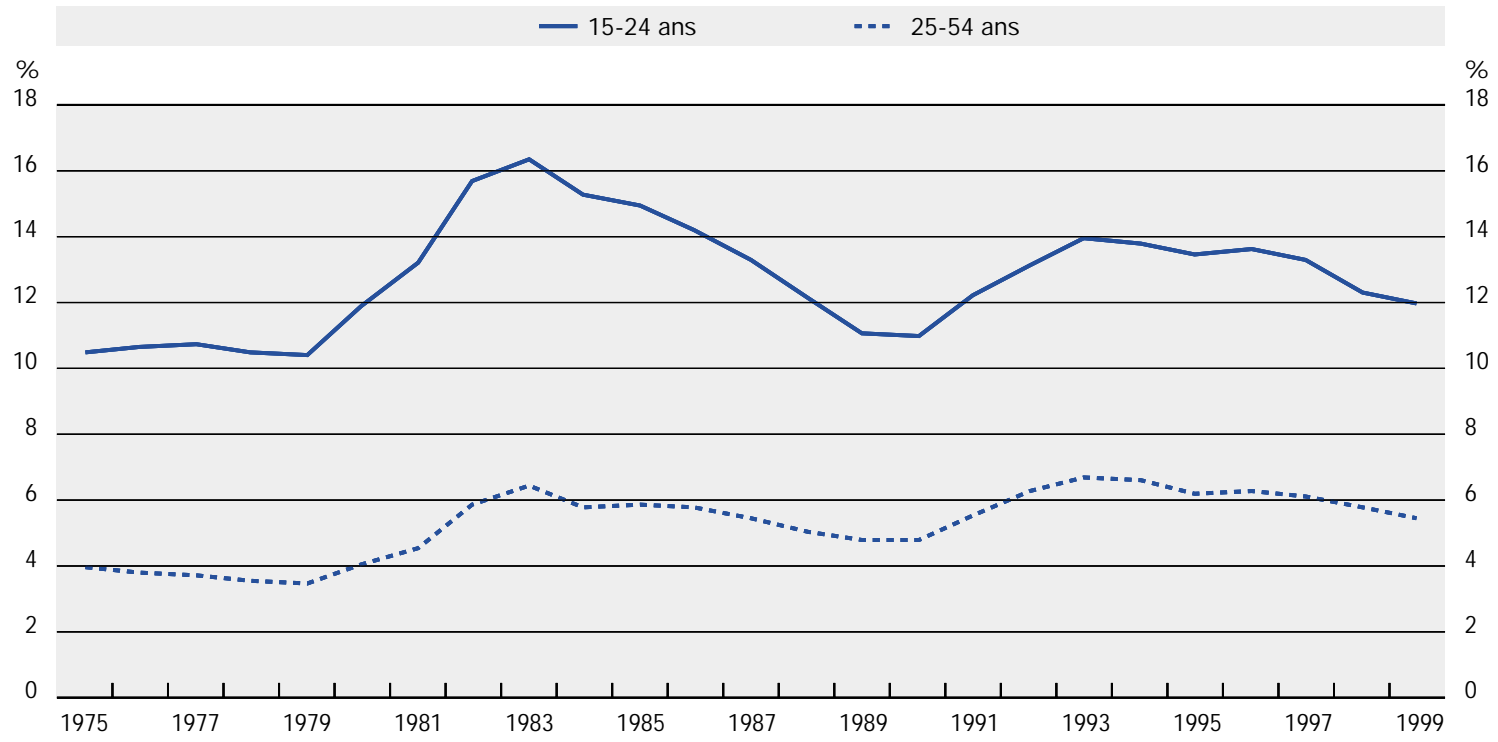

Pays pris en compte : Allemagne, Australie, Canada, Espagne, États-Unis, Finlande, France, J apon, Norvège, Pays-Bas, Portugal, Suède.

Source: OCDE (2000) à partir des enquêtes sur la population active. Le taux moyen de chômage est défini comme étant le pourcentage des chômeurs divisé par l'ensemble de la population active.

\section{Graphique B.5. Taux de dépendance dû à l'âge dans les pays de l'OCDE, 1950-2050}

-. - Moins de 16 ans et plus de 65 ans/16-64 ans _ _ Plus de 65 ans/16-64 ans

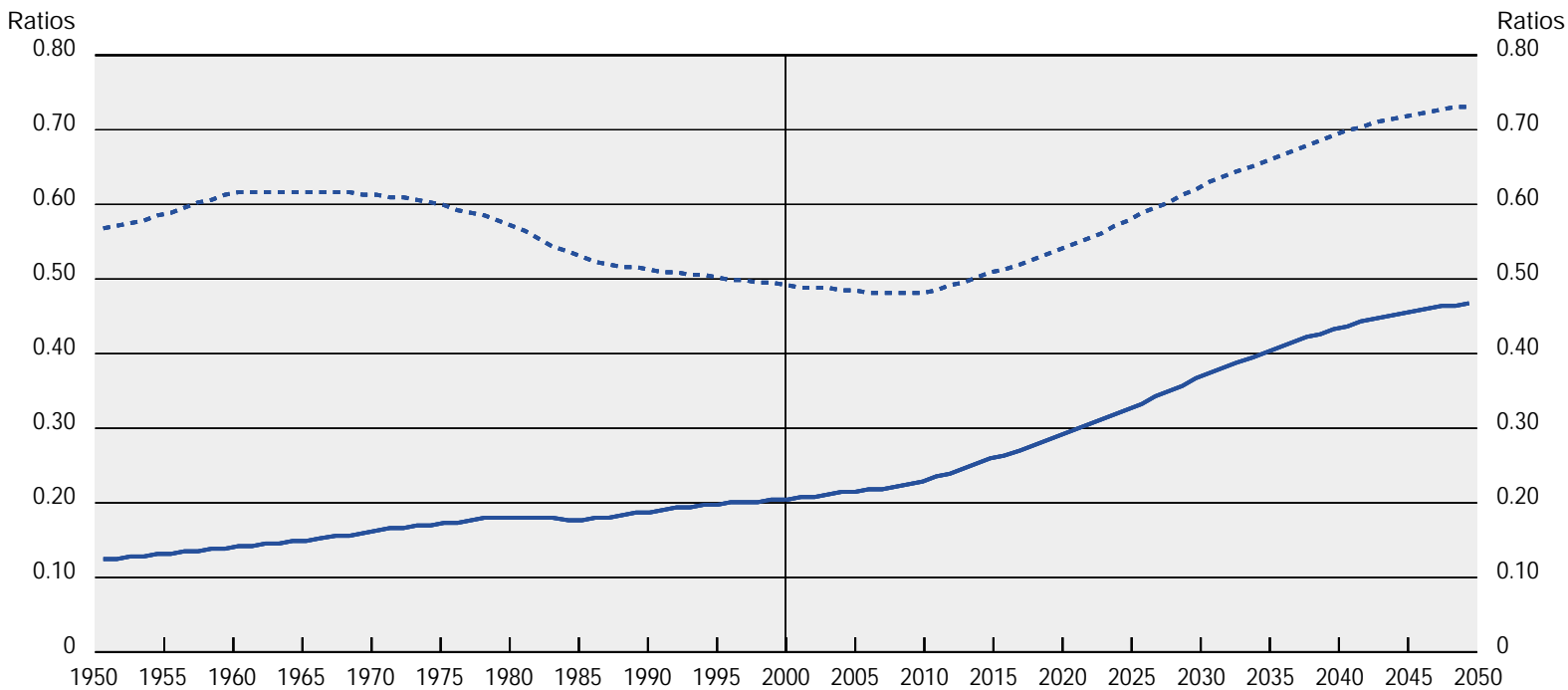

Taux de dépendance : La courbe supérieure exprime le rapport de la proportion de jeunes de moins de 16 ans et des personnes de plus de 65 ans à la population active. La courbe inférieure indique le rapport de la proportion des plus de 65 ans à la population d'âge actif.

Source: OCDE (1999), Pour un monde solidaire : le nouvel agenda social ; Nations Unies (révision 1998), Perspectives de la population mondiale 1950-2050, variante médiane. 
Graphique B.6. Pourcentage de la population des pays de l'OCDE de 25 à 64 ans ayant un niveau égal ou supérieur à la fin des études secondaires, 1950-98

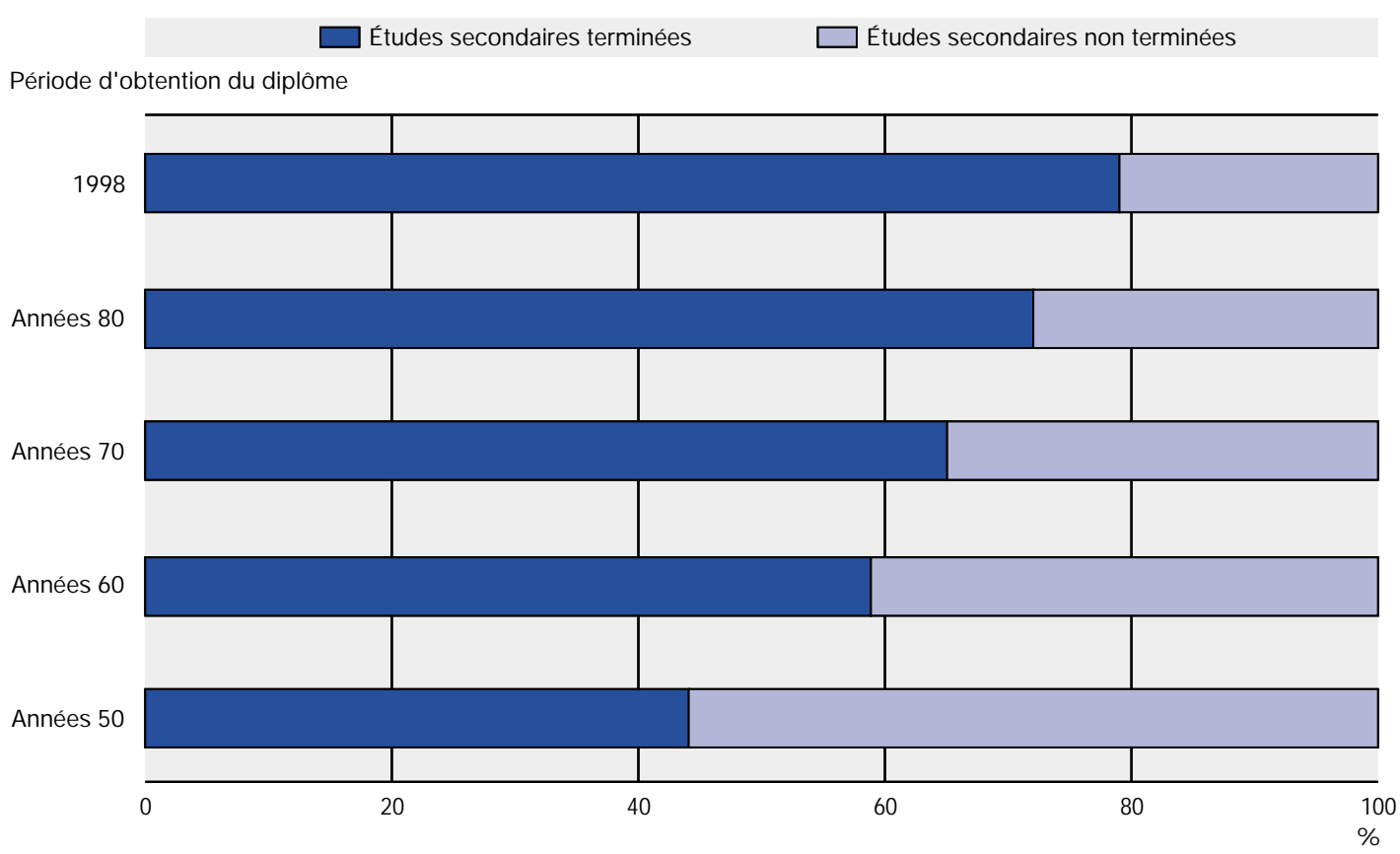

Source: OCDE (2000), Regards sur l'éducation - Les indicateurs de l'OCDE, tableaux C2.2 et C4.2.

Graphique B.7. Parents isolés dans certains pays de l'OCDE, comparaison entre les années 80 et 90 Pourcentage de ménages avec enfants comportant un seul adulte

Années $90 \quad \square$ Années 80

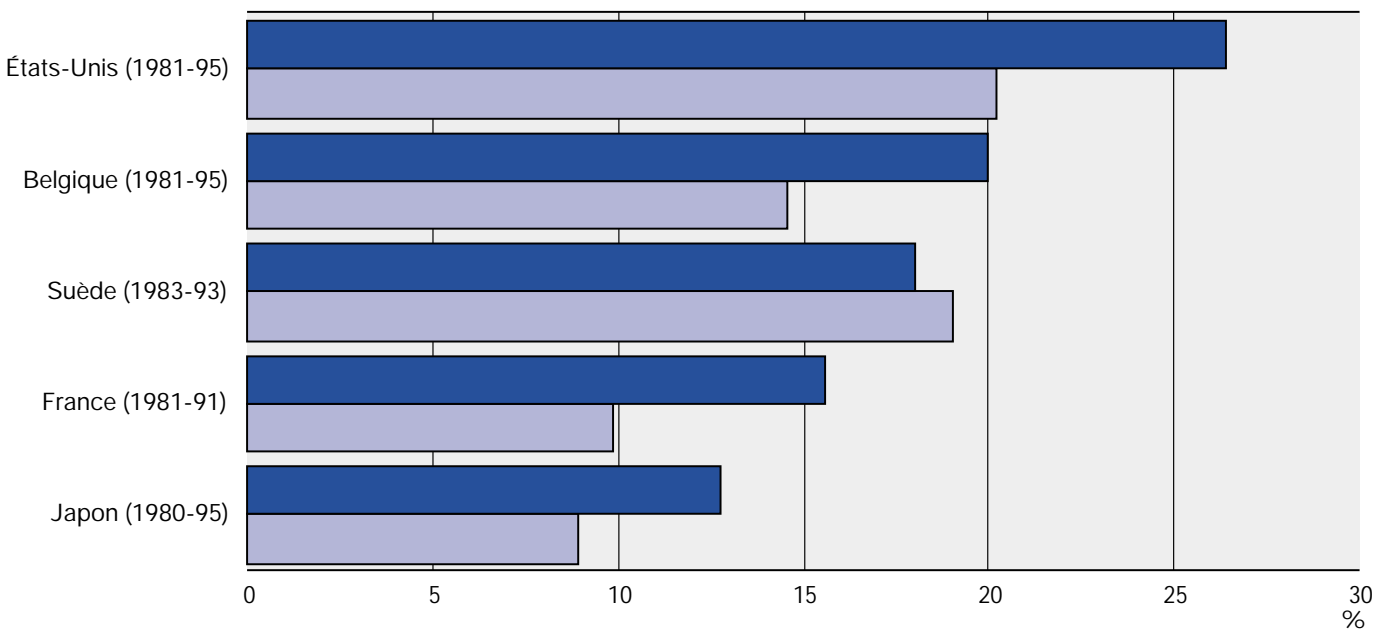

Note: Années d'enquête entre parenthèses.

Source: OCDE d'après Eurostat Demographics 1996. Le taux de parents isolés est le pourcentage de ménages avec enfants comportant un seul adulte. Le taux est défini comme le nombre de familles monoparentales en pourcentage de l'ensemble des familles ayant des enfants à charge. 
Graphique B.8. Écart de rémunération entre les hommes et les femmes de 25 à 64 ans (travaillant à plein temps), années 80 et 90

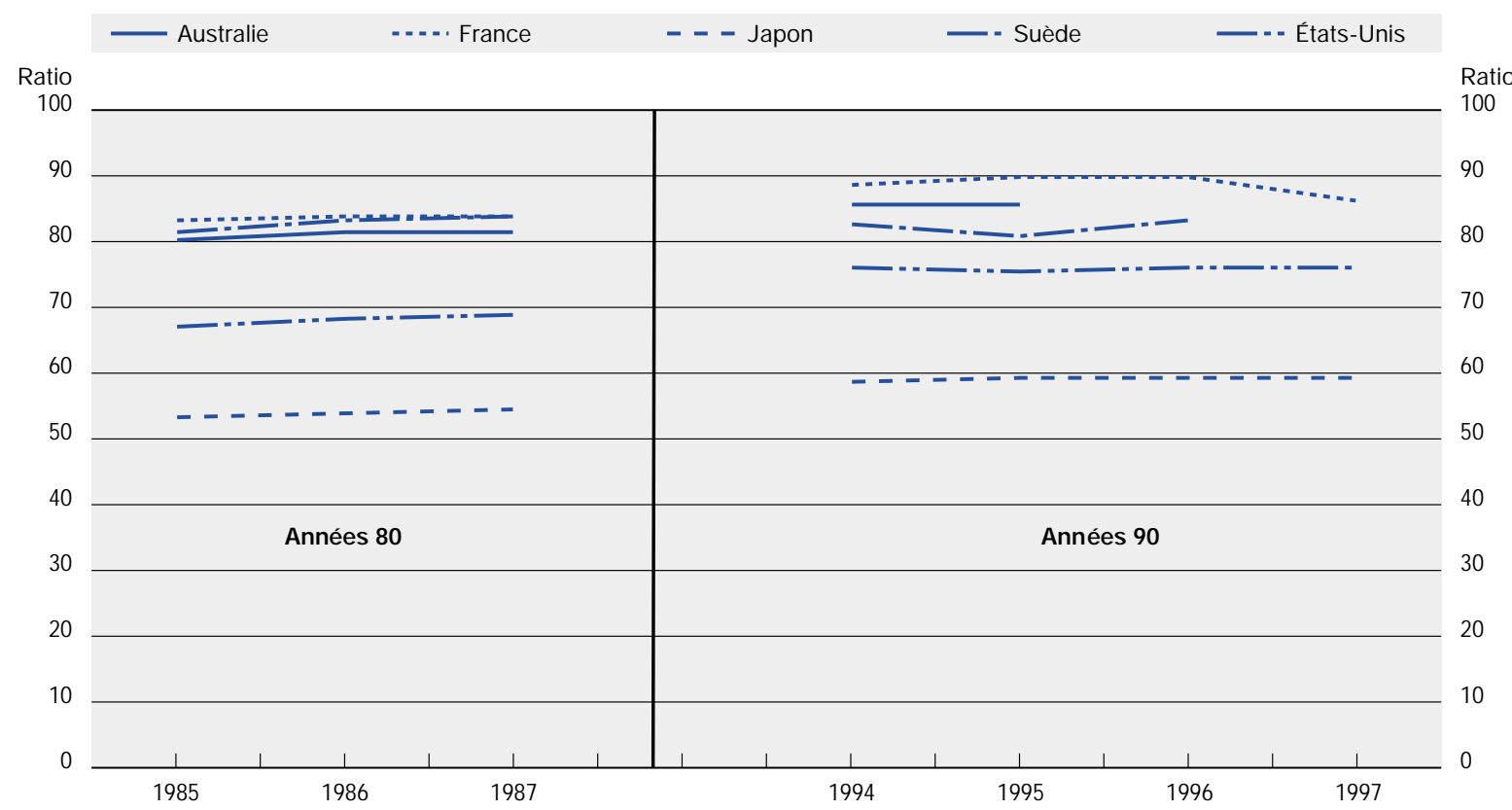

Source: Base de données de l'OCDE sur la structure des salaires, voir Keese et Puymoyen (à paraître).

Graphique B.9. Différence de taux d'activité entre les hommes et les femmes de 15 à 64 ans dans certains pays de l'OCDE, 1960-99

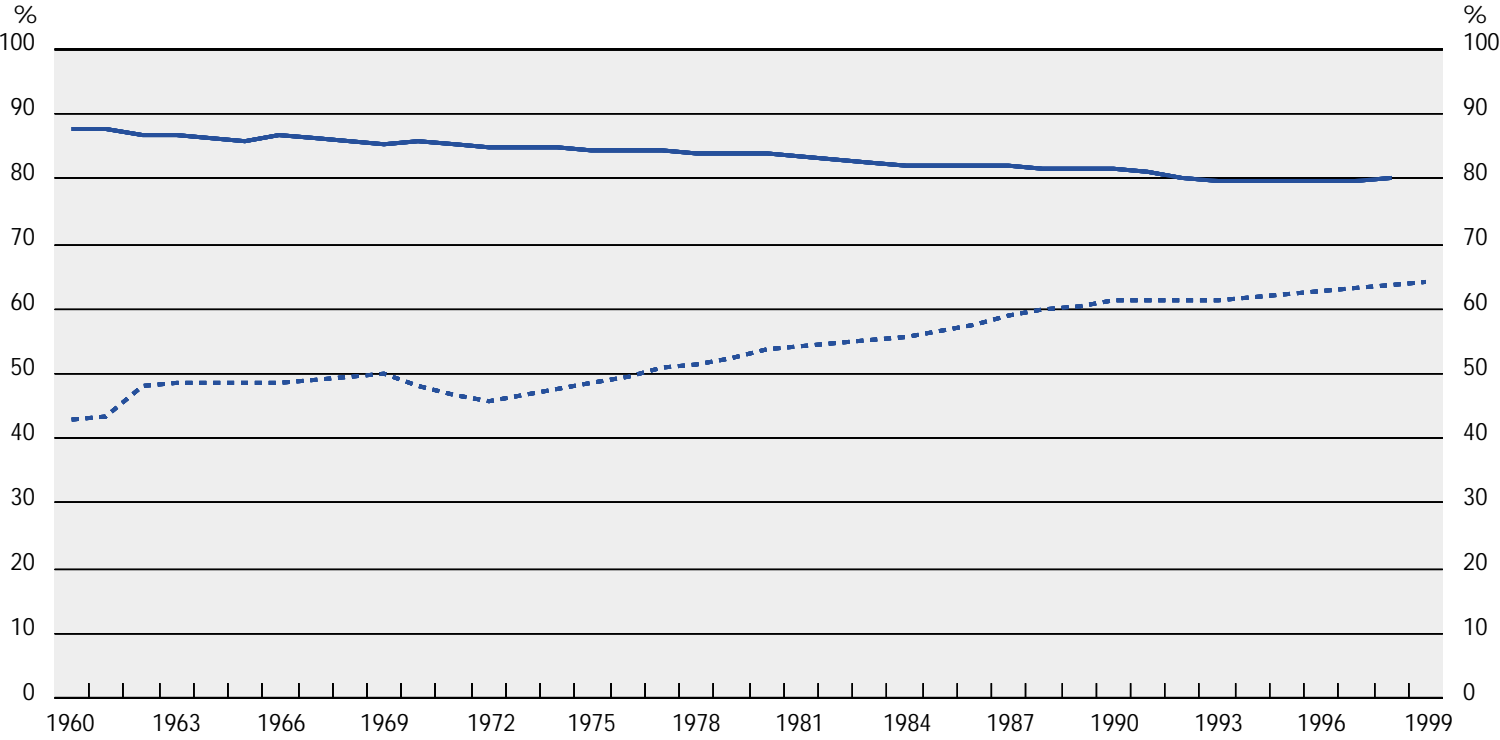

Source: OCDE d'après les enquêtes sur la population active. Moyenne basée sur l'Allemagne, l'Australie, le Canada, l'Espagne, les États-Unis, la Finlande, la France, l'Italie, le J apon, la Norvège, les Pays-Bas, le Portugal, la Suède. 
Graphique B.10. Flux d'entrées d'étrangers dans quelques pays de l'OCDE, 1999

Pour 1000 habitants

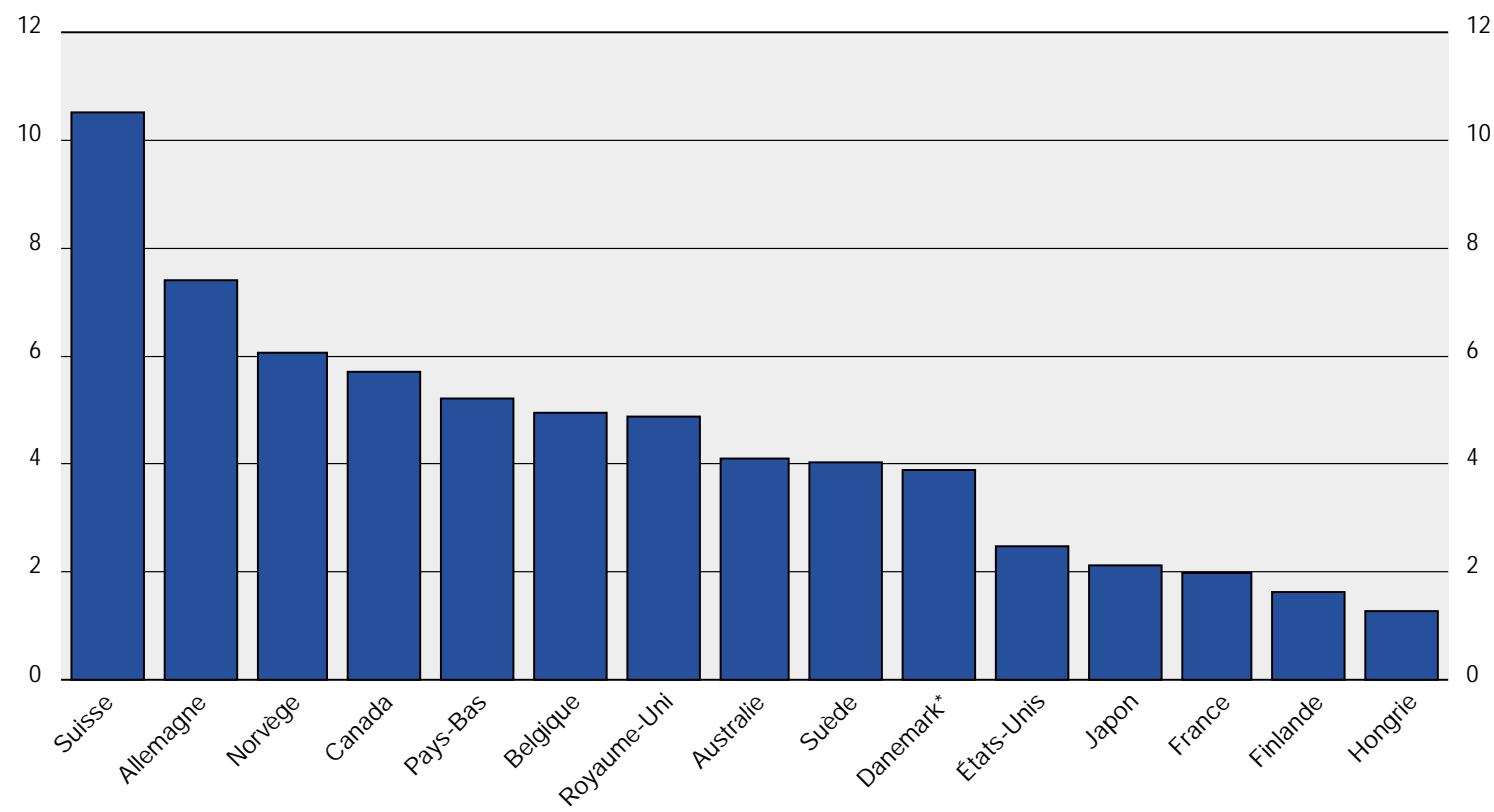

* Les données pour le Danemark se réfèrent à l'année 1998.

Source: Données de l'OCDE sur les migrations internationales ; ISTAT (1999), rapport annuel.

Graphique B.11. Nombre de décès par toxicomanie enregistrés dans l'Union européenne par million d'habitants, 1986-97

25

20

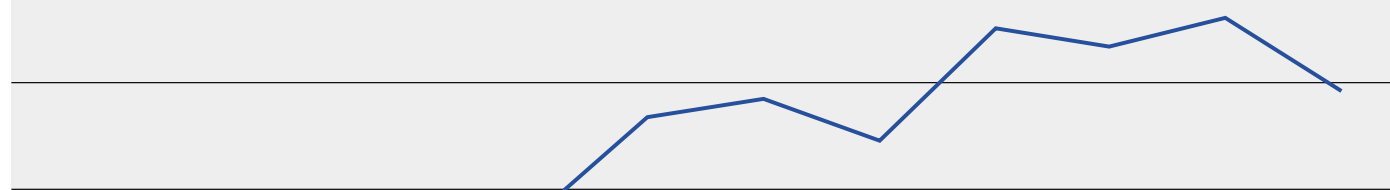

15

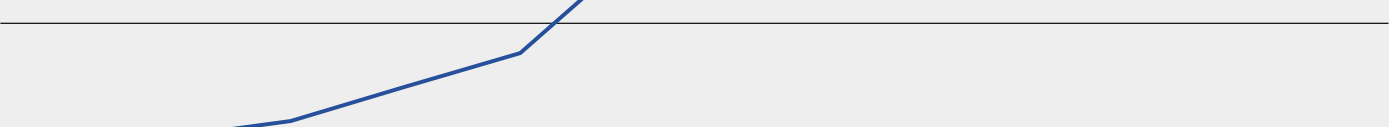

10
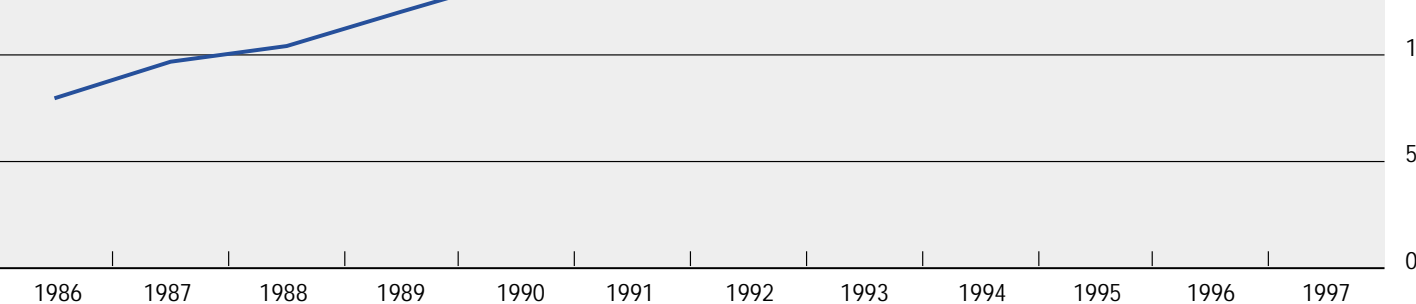

Pays pris en compte : Allemagne, Belgique, Danemark, Espagne, France, Grèce, Irlande, Italie, Luxembourg, Pays-Bas, Portugal, Royaume-Uni, Suède.

Source : Conférence des ministres européens sur les drogues et la toxicomanie ; UNDPC Redbook (2000), Global Illicit Drugs Trends ; projections démographiques des Nations Unies (révision 1998). 
Graphique B.12. Pourcentage de la population victime de délinquance, années 80 et 90

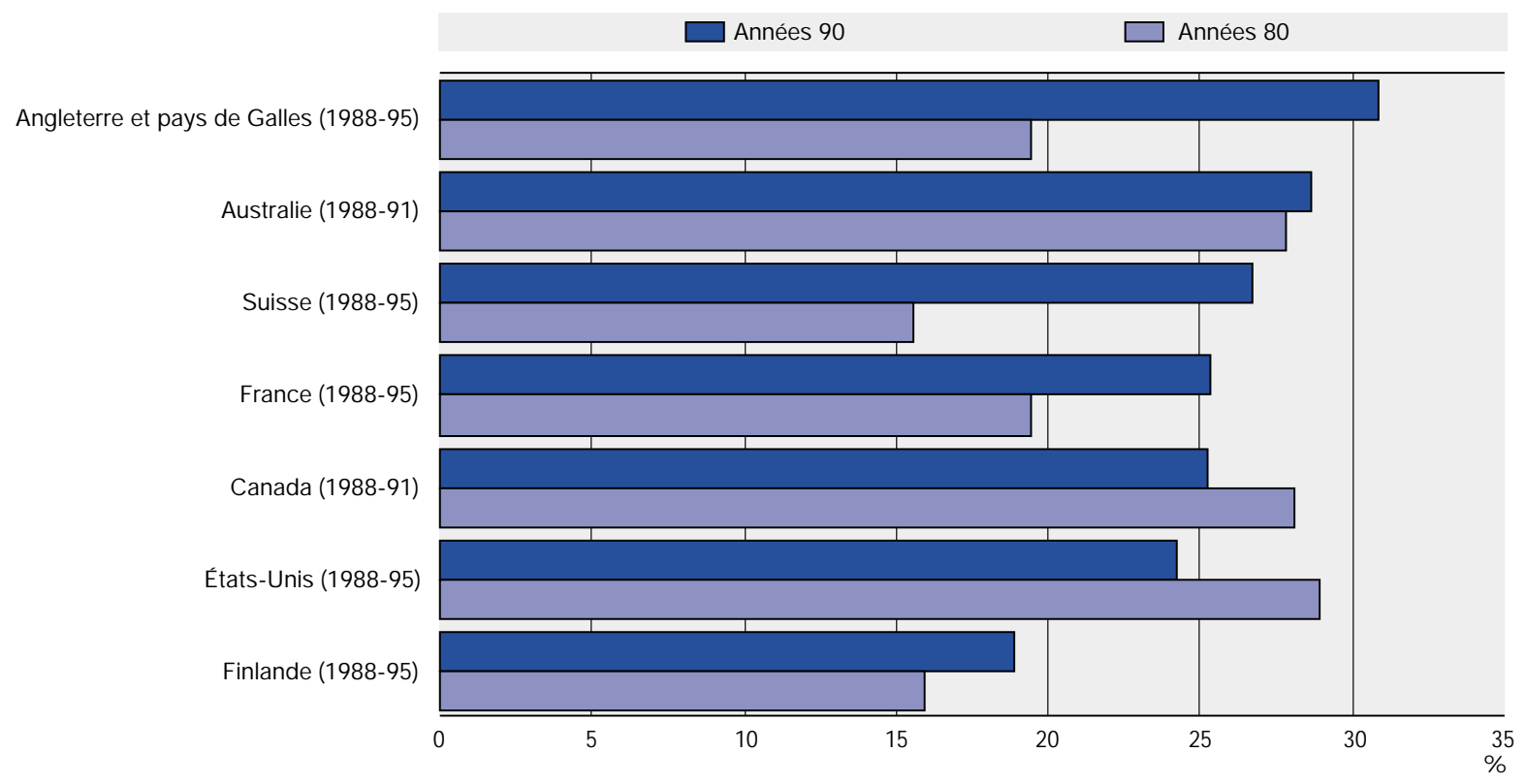

Note: Pourcentage de la population victime de délinquance au cours d'une année. Année d'enquête entre parenthèses.

Source : 1996 International Crime Victims Survey.

Graphique B.13. Espérance moyenne de vie à la naissance dans certains pays de l'OCDE, 1960-98

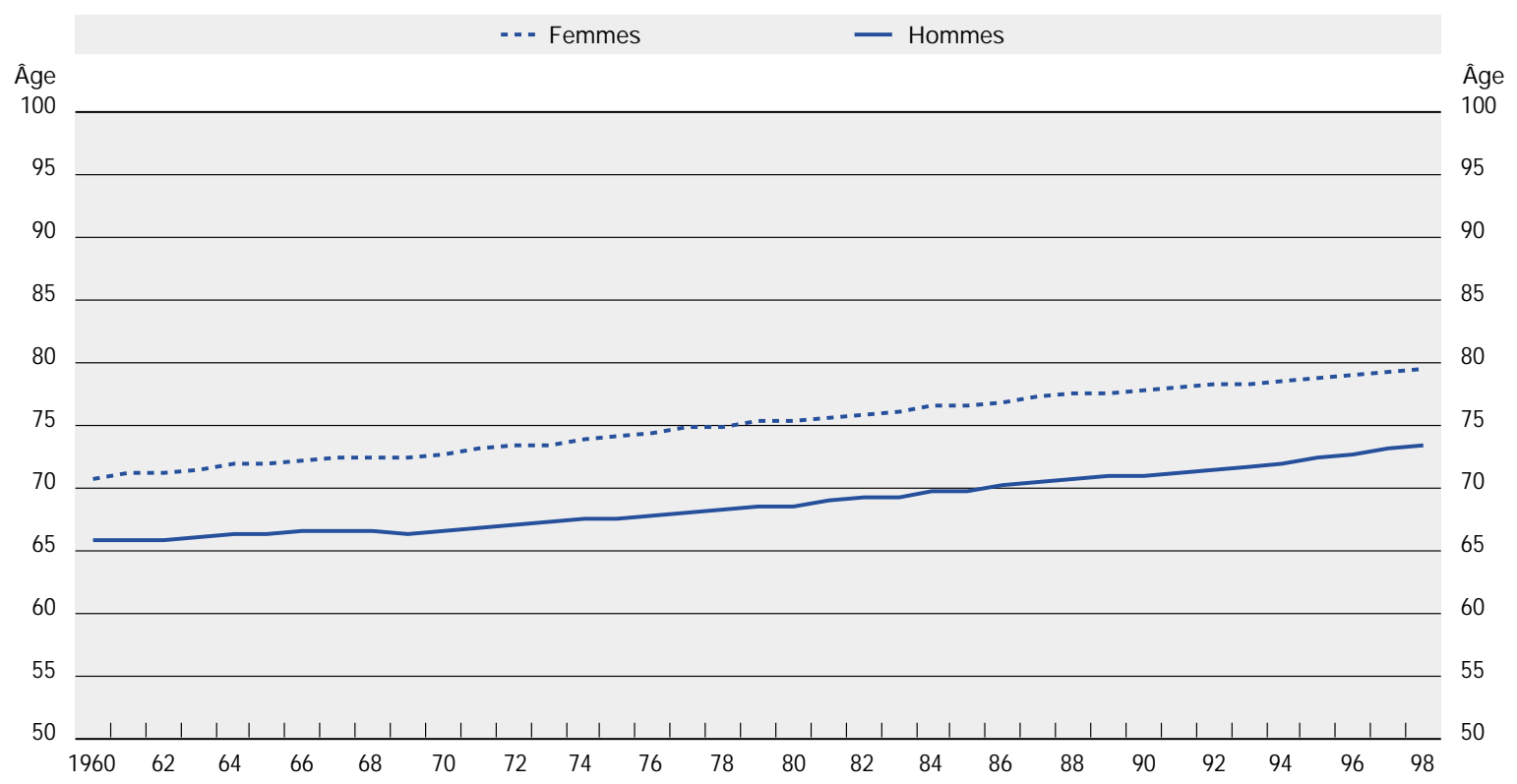

Pays pris en compte : Allemagne, Australie, Autriche, Belgique, États-Unis, France, Hongrie, J apon, Mexique, Norvège, Nouvelle-Zélande, Pays-Bas,

Source: OCDE (2000), Éco-Santé OCDE. 


\section{Graphique B.14. Tendances récentes de la satisfaction personnelle dans 12 pays de l'Union européenne, 1973, 1983 et 1997}

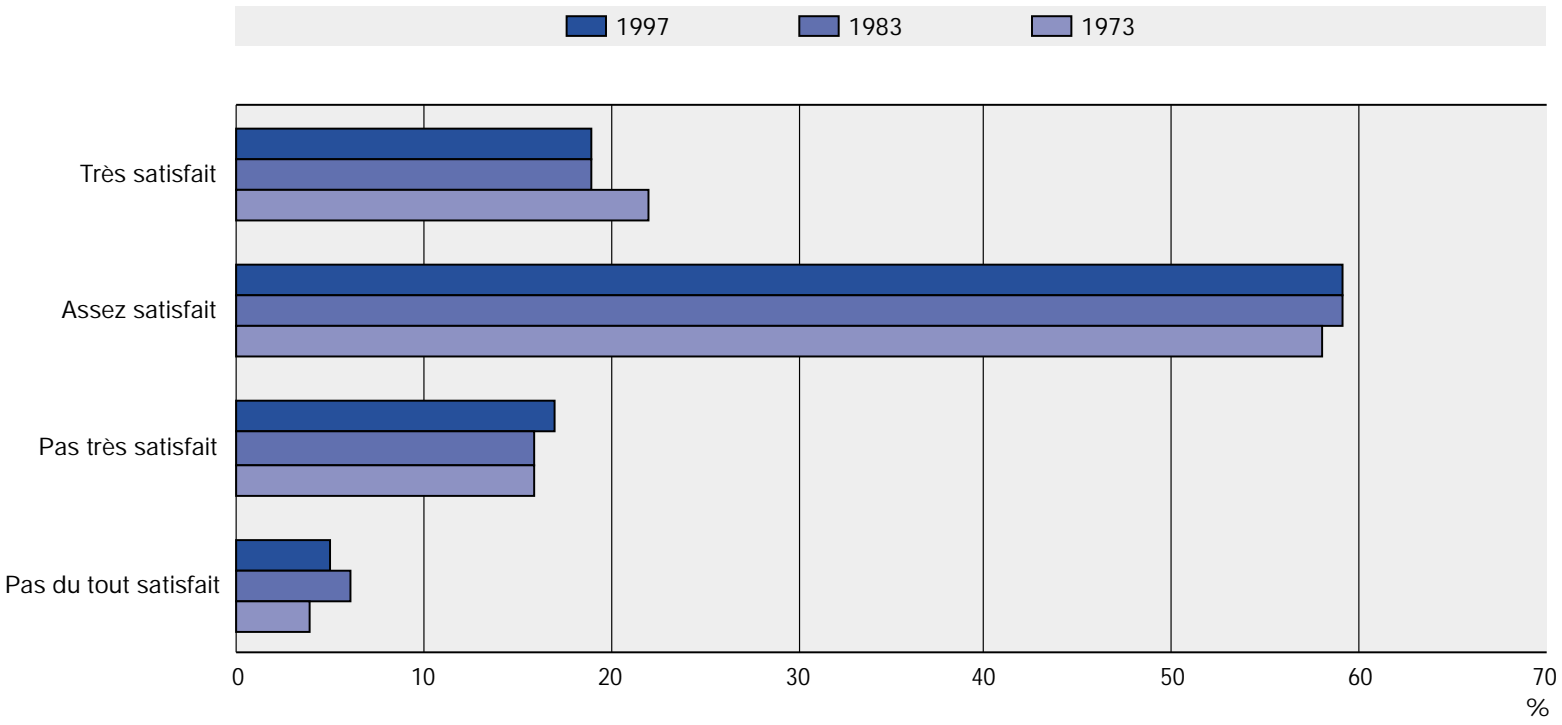

Note: Pays pondérés selon leur taille relative : Allemagne de l'Ouest, Belgique, Danemark, Espagne, France, Grèce, Irlande, Italie, Luxembourg, Pays-Bas, Portugal, Royaume-Uni.

Source : Fichier cumulatif des Eurobaromètres (ICPCR n॰9361) de 1973 à 1983 et Eurobaromètre n॰ 47.1 (ICPCR n॰2089) d'avril 1997 cités dans Blanchflower et Oswald (2000).

Graphique B.15. Proportion de personnes donnant différentes réponses sur le bonheur, États-Unis, 1972-98

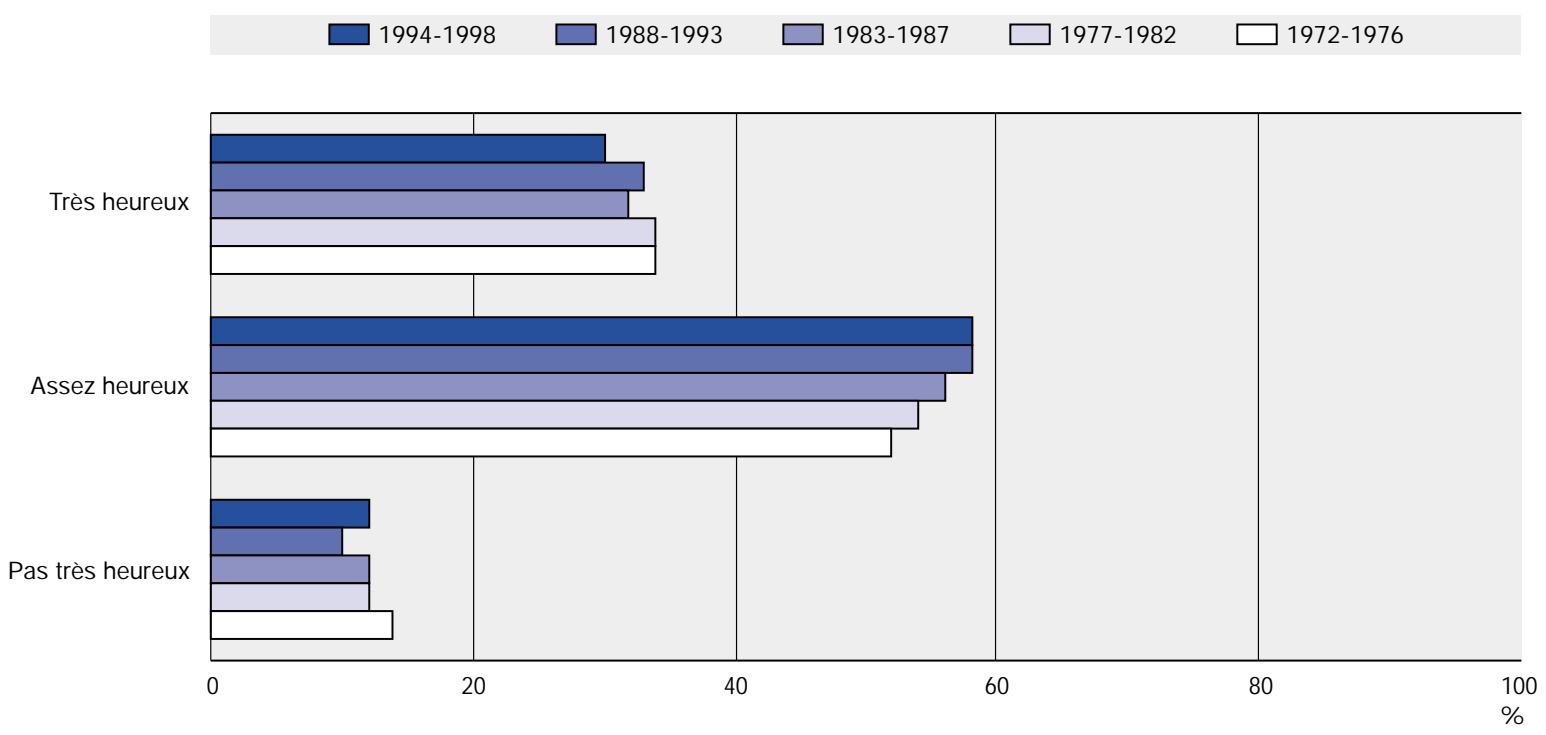

Source : US General Social Survey cité dans Blanchflower et Oswald (2000). 


\section{Graphique B.16. Taux de suicide dans certains pays de l'OCDE, 1950-97}

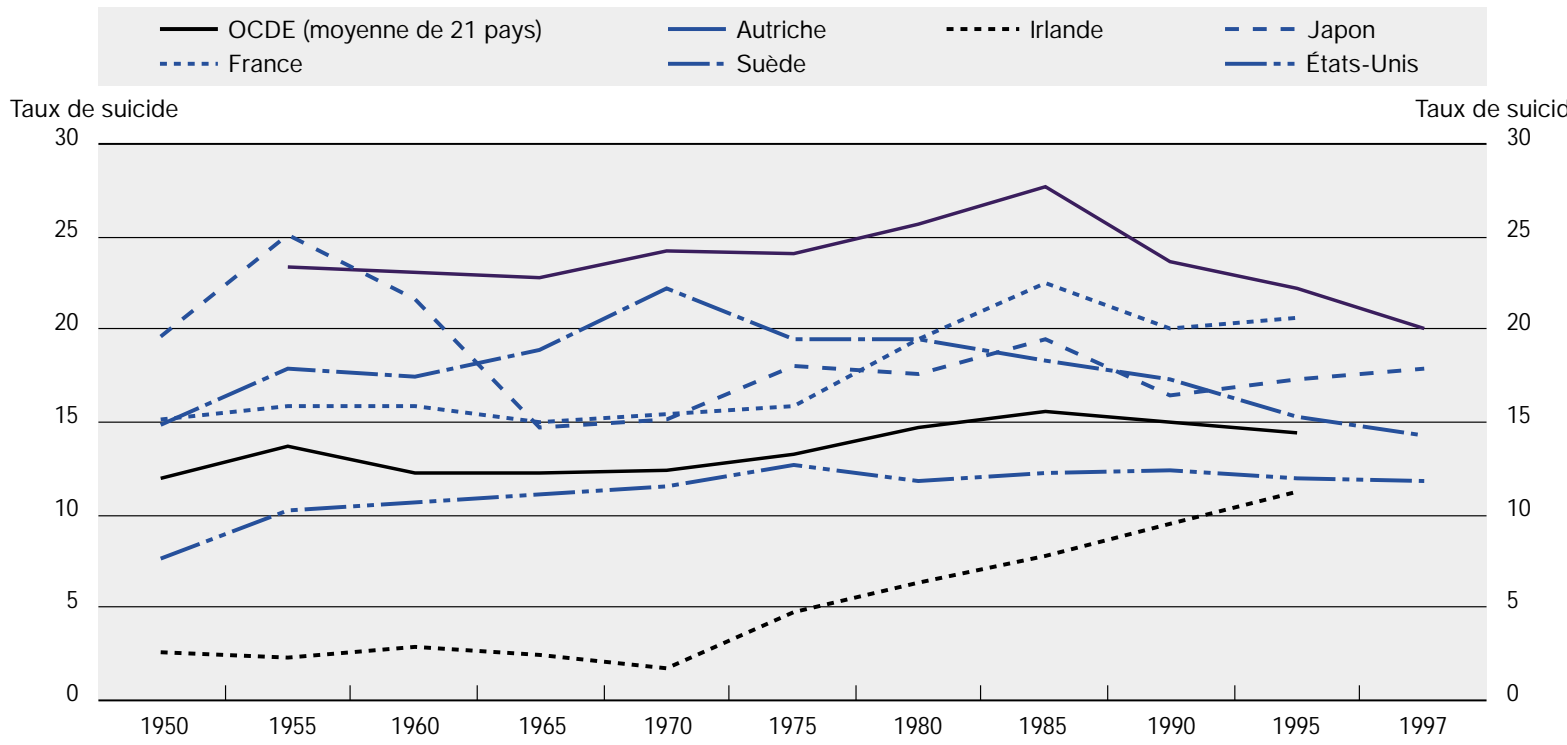

Note: La moyenne des 21 pays comprend : Australie, Autriche, Belgique, Canada, Danemark, Espagne, États-Unis, Finlande, France, Grèce, Irlande, Islande, Italie, J apon, Luxembourg, Nouvelle-Zélande, Pays-Bas, Pologne, Portugal, Suède, Suisse.

Source: Organisation mondiale de la santé.

Graphique B.17. Émissions de gaz à effet de serre dans les pays de l'OCDE selon différents scénarios, 1990-2010

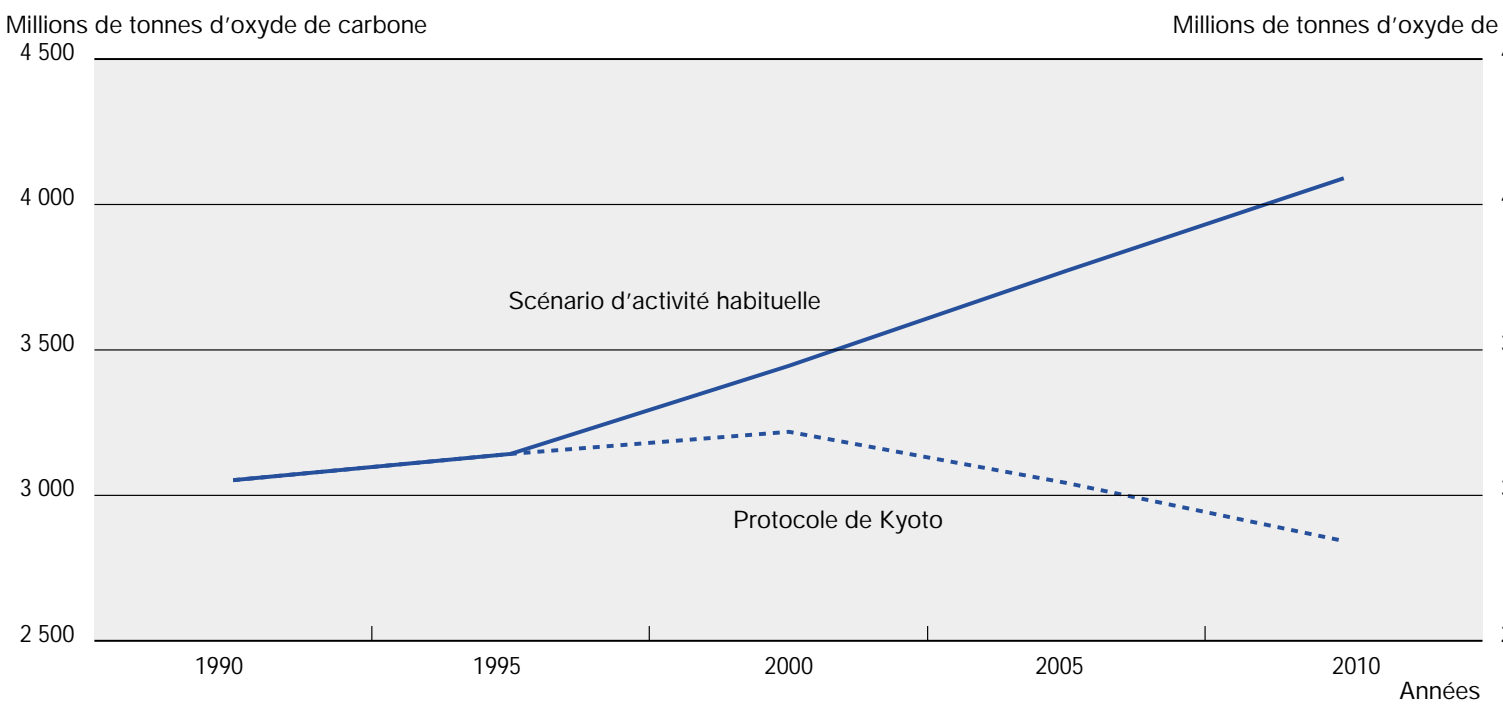

Note: La tendance du «Protocole de Kyoto » est basée sur le protocole de Kyoto de 1997 selon lequel les pays industriels se sont engagés à des réductions des émissions de gaz à effet de serre à partir des niveaux de 1990 sur la période 2008-2012. Le scénario « d'activité habituelle » est une projection si les conditions actuelles demeuraient inchangées dans le futur.

Source : OCDE. Projections des émissions de gaz à effet de serre selon le modèle VERT de l'OCDE. 


\section{Annexe C}

\section{DÉTERMINANTS DU NIVEAU SCOLAIRE : LES RÉSULTATS DES RECHERCHES}

Cette annexe décrit les recherches récentes (résumées dans la section 2.4 du chapitre 2 ) sur les déterminants du niveau scolaire. Les données portent pour la plupart sur les États-Unis et uniquement sur une série limitée d'acquis scolaires. Toutefois, elles tendent effectivement à montrer que le milieu familial et l'environnement immédiat sont des déterminants essentiels des résultats scolaires et peuvent contribuer puissamment à élever le niveau, tout comme les stratégies visant à améliorer la qualité des pratiques pédagogiques. Des financements accrus et ciblés, permettant notamment de réduire la taille des classes, peuvent aussi favoriser une amélioration des performances s'ils se conjuguent avec une gestion des ressources et des pratiques pédagogiques plus efficaces. Indépendamment de l'incidence des moyens matériels mis en œuvre et des changements apportés aux méthodes d'enseignement et d'ap prentissage, les recherches donnent à penser que les normes et les réseaux sociaux continuent de faciliter sensiblement la formation à tous les stades de la vie.

Coleman et al. (1966), rendant compte des conclusions d'une grande enquête menée au milieu des années 60 dans des écoles auxÉtats-Unis, ont constaté peu d'éléments permettant de dire que les moyens matériels mis en œuvre ainsi que la taille des classes sont liés au niveau scolaire. (Des données plus récentes révèlent une corrélation plus positive, mais mettent aussi en évidence des lacunes dans l'état des recherches actuelles et passées.) Coleman a constaté que les moyens matériels mis en œuvre dans les établissements scolaires n'influent que légèrement sur les résultats des élèves, surtout en regard du milieu familial. Toutefois, la corrélation observée varie beaucoup selon le type d'acquis évalués et selon le niveau de développement économique et social des populations considérées. Certains acquis, en sciences et en mathématiques notamment, sont plus étroitement liés à l'effet de l'école. Les compétences relationnelles et surtout les aptitudes propres dépendent sans doute beaucoup plus des parents ainsi que du milieu familial et social et moins de l'école. Autre difficulté rencontrée pour distinguer l'effet du milieu familial de l'effet de l'école, il est rarement possible, dans les pays de l'OCDE, d'observer l'effet de la non fréquentation scolaire. Fuller et Clarke (1994), en revanche, évoquent l'éventualité d'une exagération de l'effet de l'école si les mesures indirectes de l'influence du milieu familial et social sont inadéquates ou mal définies.

D'après les données, la réduction de l'effectif par classe n'a pas toujours un impact positif sur les acquis des élèves. Hanushek (1998), qui a examiné presque 300 évaluations différentes de l'effet de la réduction de l'effectif par classe sur les résultats scolaires, affirme que rien n'indique une corrélation positive entre une réduction généralisée de la taille des classes et le degré moyen d'amélioration des résultats des élèves. Sur les 277 études de l'impact du taux d'encadrement qu'il a passées en revue, Hanushek constate que, dans $15 \%$ des cas, ce ratio a une incidence positive et statistiquement significative sur les performances scolaires tandis que dans un peu moins de $5 \%$ des cas, la corrélation est négative. Selon observations faites, une réduction généralisée de l'effectif par classe n'entraînera pas nécessairement un relèvement des résultats - peut-être pas tout au moins s'il n'y a pas de changements dans les méthodes pédagogiques, la motivation des élèves et l'influence du milieu familial.

En revanche, d'après les conclusions d'une expérience de "répartition aléatoire » des élèves, menée à très grande échelle, au milieu des années 80, dans l'État américain du Tennessee (le projet STAR1), la réduction de la taille des classes à l'école maternelle ou dans les petites classes de l'enseignement élémentaire entraîne une légère amélioration des résultats scolaires2. Dans les classes de niveau plus élevé, l'amélioration semble beaucoup moins perceptible, si tant est qu'elle le soit. Toutefois, utilisant des résultats du projet STAR et d'autres travaux de recherche, Mosteller (1995) a constaté que cette réduction profite plus aux élèves issus des milieux défavorisés qu'aux autres, 
ce qui tend à prouver qu'une réduction ciblée de l'effectif par classe pourrait être un moyen de lutter contre les inégalités sociales. Exploitant eux aussi les données du projet STAR, Nye et Hedges (2000) signalent également que la taille de la classe a un effet important. D'une manière plus générale, d'autres recherches vont être nécessaires pour déterminer dans quelle mesure l'effet relatif de la taille des classes explique les résultats des élèves, tous niveaux d'enseignement confondus. Dans les pays mêmes, la taille des classes (ou le taux d'encadrement dans le secondaire et, surtout, le supérieur) varie considérablement selon le niveau d'enseignement (du primaire au supérieur), le domaine d'études, le type d'établissement (enseignement normal ou spécial) ainsi que le lieu d'implantation (milieu urbain ou rural). Les possibilités de modifier l'effectif par classe et les conséquences susceptibles d'en découler varient suivant le type d'enseignement et le groupe cible considérés.

Les données révèlent de grands écarts de résultats au sein des établissements scolaires et entre eux ainsi que des variations dans l'efficacité des pratiques pédagogiques dans les écoles. Dans les écoles mêmes, la progression des acquis d'une classe à la classe suivante varie beaucoup, semble-t-il, en fonction de la qualité des enseignants (Hanushek, Kain et Rivkin, 1998). Hanushek (1992) montre que, selon que l'enseignant maîtrise ou non sa classe, les acquis des élèves peuvent représenter plus d'une année et demi d'études d'écart en une seule année scolaire. Les enseignants se différencient systématiquement par la rapidité avec laquelle leurs élèves ap prennent (Murnane, 1975), même si l'on tient compte des caractéristiques personnelles des élèves. De plus, Rivkin, Hanushek et Kain (1998) ont constaté que les différences de qualité d'un enseignant à l'autre sont plus significatives à l'intérieur d'un établissement qu'entre les établissements d'un district ou d'une région donné. De même, des études sur le devenir des diplômés révèlent que les différences de performances sur le marché du travail sont liées à la qualité de l'établissement fréquenté (Betts, 1995 et Grogger, 1996).

D'autres recherches ont été consacrées aux effets qu'a sur les résultats scolaires la concurrence entre écoles - que les parents aient un choix plus large dans une localité ou qu'il existe des dispositifs de type chèque-éducation permettant aux parents «d'acheter » les services d'enseignement de l'organisation de leur choix au moyen de ce chèque financé sur fonds publics. Toutefois, les systèmes de chèque-éducation et leurs répercussions continuent de faire l'objet d'un vif débat aux États-Unis et les recherches sur les résultats obtenus n'ont pas encore abouti. Hoxby (1994) laisse entendre que la présence d'écoles privées dans une région semble avoir pour effet d'améliorer l'efficience des écoles publiques voisines, qui se trouvent alors en concurrence avec elles pour attirer les élèves. Shleifer (1998) montre que l'existence d'écoles privées, conjuguée à la possibilité de choix et à la concurrence, favorise puissamment une diminution des coûts et une amélioration des performances scolaires. L'une des grandes questions est cependant de savoir quelles sont les conséquences, pour l'égalité, des changements introduits en matière de concurrence et de choix parental d'établissement eu égard à l'inégalité des chances et aux différences de dotations en moyens financiers et en capital social. Quant à savoir si ces innovations aux États-Unis comme ailleurs améliorent la qualité sans compromettre l'équité, cela reste à voir. Par ailleurs, les études portant sur différents types d'arrangements contractuels, et notamment sur les performances des écoles privées et des écoles publiques, sont limitées par l'absence de contrôles adéquats et par des facteurs perturbateurs 3 .

Certaines données internationales montrent que le fait de centraliser les examens ainsi que les mécanismes de contrôle du respect des normes et des budgets au niveau national ou régional peut contribuer à améliorer les performances des écoles 4 (Wossmann, 2000 et Bishop, 1999). De même, une véritable délégation des pouvoirs de décision aux écoles ou au niveau d'administration local, surtout dans certains domaines de compétences tels que la politique du personnel et son recrutement, ainsi que pour l'achat des supports pédagogiques peut aider les écoles à améliorer leurs performances 5 (Wossman, 2000).

Les observations tirées de diverses études couvrant un large éventail de pays (et résumées dans Fuller et Clarke, 1994) conduisent à penser que le fait, pour les élèves, de disposer de manuels scolaires et d'autres ouvrages ainsi que de bibliothèques scolaires a un impact significatif sur les résultats obtenus, surtout dans le primaire. Toutefois, la fréquence et le type d'usage qui est fait de ces matériels jouent sans doute un rôle plus déterminant que la simple existence d'une bibliothèque dans l'école.

Peu d'analyses ont été faites de l'influence de leurs condisciples sur les résultats obtenus par les élèves, et les rares études dont on dispose sont sujettes à caution du fait de la difficulté rencontrée pour déterminer ces effets en tant que tels. Hanushek, Kain, Markman et Rivkin (2000) ont examiné les résultats des élèves du primaire (à l'issue 
de tests en calcul et en sciences) dans l'État du Texas, et ont montré que le niveau des résultats et la composition raciale du groupe de leurs condisciples influent directement sur les résultats ob tenus par les élèves6. II semble que tous profitent du fait d'avoir des camarades qui obtiennent de bons résultats bien que l'effet produit ne soit pas très marqué.

Comme on l'a dit dans le chapitre 2 plus haut, le temps que les parents passent avec leurs enfants et la qualité de leur présence sont essentiels. Ce que les parents font avec leurs enfants semble plus important que leur situation socio-économique. Ainsi, selon une étude, la participation des parents à la vie familiale a un effet plus important que le bénévolat à l'école ou la participation à l'administration de l'établissement (Ho Sui-Chu et Willms, 1996). Ces auteurs ont également noté que les indicateurs de l'investissement parental sont plus parlants que ceux du milieu social d'origine - ce qui renforce l'argument selon lequel l'effet du milieu social peut être considérablement atténué selon le degré d'investissement des parents et leur comportement. Carlson (1999) a observé, à partir de données longitudinales américaines, que le fait pour les pères biologiques (même s'ils ne vivent pas auprès d'eux) de participer activement à la vie de leurs enfants peut avoir des incidences positives très diverses sur le comportement des adolescents, y compris leur assiduité à l'école, incidences qui vont bien au-delà des effets de la structure familiale.

Certaines études ont plus particulièrement porté sur l'étendue et la qualité des réseaux associant les parents, les enseignants et la communauté locale. Coleman cite, à titre d'exemple, les réseaux de capital social autour des écoles, les efforts et le temps consacrés par les parents à développer des relations étroites avec leurs enfants, les enseignants et les camarades de leurs enfants, les parents de ces camarades ainsi qu'avec d'autres adultes jouant un rôle important dans la vie des enfants. La confiance mutuelle entre les différents éléments d'un réseau instaure un climat de soutien et permet la surveillance et l'influence sociale. Les parents ont ainsi la possibilité de s'investir davantage, de mieux connaître les camarades, les activités et les progrès scolaires de leurs enfants. Une étude de Coleman et Hoffer sur les écoles confessionnelles, privées et publiques des États-Unis leur a permis de constater que, dans les premières, le taux d'abandon des études était nettement inférieur à ceux qu'enregistraient les écoles publiques ou les établissements privés non confessionnels (Coleman et Hoffer, 1987). Ces deux chercheurs font valoir que les performances des écoles confessionnelles sont généralement meilleures non seulement parce qu'on y applique des normes et des préceptes propices à l'apprentissage et à l'enseignement de qualité, mais aussi à cause de l'effet de « convergence sociale » de l'école et des réseaux communautaires au sens large, qui rapprochent les parents des élèves. En tant que communautés d'apprentissage, les écoles où les relations de confiance sont les plus étroites obtiennent de meilleurs résultats même si l'on tient compte de différents autres facteurs, dont la formation des enseignants et les caractéristiques des élèves (Bryk, Lee et Holland, 1993, pp. 314).

S'agissant de l'accueil et de l'éducation de la prime enfance à l'extérieur du foyer, les partenariats entre les prestataires de services et les familles peuvent renforcer non seulement le développement des enfants mais également les compétences des parents et l'estime qu'ils ont d'eux-mêmes (Powell, 1989). La participation au développement effectif des tout jeunes enfants peut également stimuler les réseaux de capital social à travers, par exemple, l'organisation d'activités de formation, la mise en œuvre de programmes et de décisions dans lesquelles interviennent les parents, les personnels d'accueil et autres. En outre, tout comme l'enseignement primaire et secondaire, la prise en charge des tout jeunes enfants peut générer la mise en place de réseaux sociaux de soutien et de liens solides avec d'autres familles.

Putnam (2000a) a étudié l'influence du capital social sur l'éducation auxÉtats-Unis. Il a constaté l'existence d'une corrélation étroite et significative entre les mesures du capital social au niveau agrégé pour chaque État et la qualité des acquis. Pour ce faire, il s'est servi des résultats aux tests normalisés d'aptitudes (SAT)7. Putnam (2000a, pp. 299-230) signale que le capital social à l'échelon de l'État a un effet considérable sur les résultats individuels. II est arrivé à cette conclusion après avoir tenu compte de toute une série de variables pouvant fausser les résultats, notamment la race, le niveau de revenu et les inégalités dans ce domaine, le niveau de formation de la population adulte, les taux de pauvreté, les dépenses d'éducation, les rémunérations des enseignants, la taille des classes, la structure familiale, l'ap partenance confessionnelle ainsi que la taille du secteur de l'enseignement privé. Toutefois, même si l'on tient largement compte des effets du niveau de revenu et d'autres variables, il se peut que d'autres facteurs, passés inaperçus, se corrèlent avec les mesures de variables directes du capital social et les résultats obtenus. Ces conclusions donnent à penser que : 
- des niveaux relativement élevés d'interaction sociale semblent avoir une incidence positive considérable sur l'acquisition de connaissances ;

- les niveaux de confiance et de socialisation informelle semblent revêtir plus d'importance que les niveaux d'adhésion à des organisations et les indicateurs connexes ; et

- comparé aux moyens d'intervention classique tels que la réduction de la taille moyenne des classes, le capital social joue semble-t-il davantage pour élever les résultats obtenus.

Après avoir analysé de multiples études, Henderson et Berla (1994) concluent que... « désormais, l'évidence s'impose : lorsque les écoles collaborent avec les parents pour soutenir l'ap prentissage, en règle générale, les enfants réussissent non seulement dans leurs études, mais aussi tout au long de leur vie. Lorsque les parents aident leurs enfants à faire leurs devoirs à la maison, ceux-ci obtiennent de meilleurs résultats scolaires, et les établissements qu'ils fréquentent sont d'un meilleur niveau » (Coleman et Hoffer, 1987). Ce sont surtout les familles des régions et des milieux défavorisés multipliant les handicaps (faibles revenus, difficultés d'accès à l'emploi et aux réseaux sociaux) qui sont susceptibles de bénéficier des partenariats école-collectivité-parents visant à assurer un soutien scolaire. 


\section{Notes}

1. STAR est I'acronyme de Student-Teacher Achievement Ratio, valeur mesurée lors d'une expérience menée au Tennessee.

2. Pour connaître les conclusions du projet STAR, voir Hanushek (1998). Le projet STAR a permis de réaliser une expérience spéciale, consistant à répartir des enfants de manière aléatoire entre des classes de taille différente, de l'école maternelle jusqu'en troisième année du primaire.

3. Comme dans d'autres domaines des sciences sociales, il n'est pas facile d'effectuer des études sur des échantillons totalement aléatoires étant donné que les contrôles statistiques se limitent aux échantillons utilisés qui proviennent d'un environnement non aléatoire, d'où la difficulté d'éliminer complètement l'erreur systématique dans la sélection des groupes et des individus.

4. Bien que l'impact semble plus sensible en mathématiques qu'en sciences. Voir Wossmann, 2000.

5. En regard de l'autonomie des écoles et de l'existence d'examens et de normes centralisés, les résultats se tiennent, tant en micro-analyse, lorsqu'on utilise les données de la Troisième enquête internationale sur les mathématiques et les sciences (TIMSS) qu'en macro-analyse lorsque l'on utilise les moyennes par pays des 39 pays ayant participé à la TIMSS.

6. Pour cette étude, différentes méthodes statistiques ont été utilisées pour distinguer les effets prop res au groupe des condisciples des effets de l'école, de la collectivité et autres.

7. La mesure composite utilisée par Putnam comprend les indicateurs suivants : i) intensité de l'engagement dans la vie et l'organisation de la communauté, ii) engagement dans la vie publique (par exemple, exercice du droit de vote), iii) bénévolat, iv) sociabilité informelle (visites d'amis, par exemple), et v) degré de confiance notifié. 
Annexe D

\section{L'INCIDENCE DU CAPITAL HUMAIN SUR LA CROISSANCE ÉCONOMIQUE : QUELQUES GRANDES ÉTUDES}




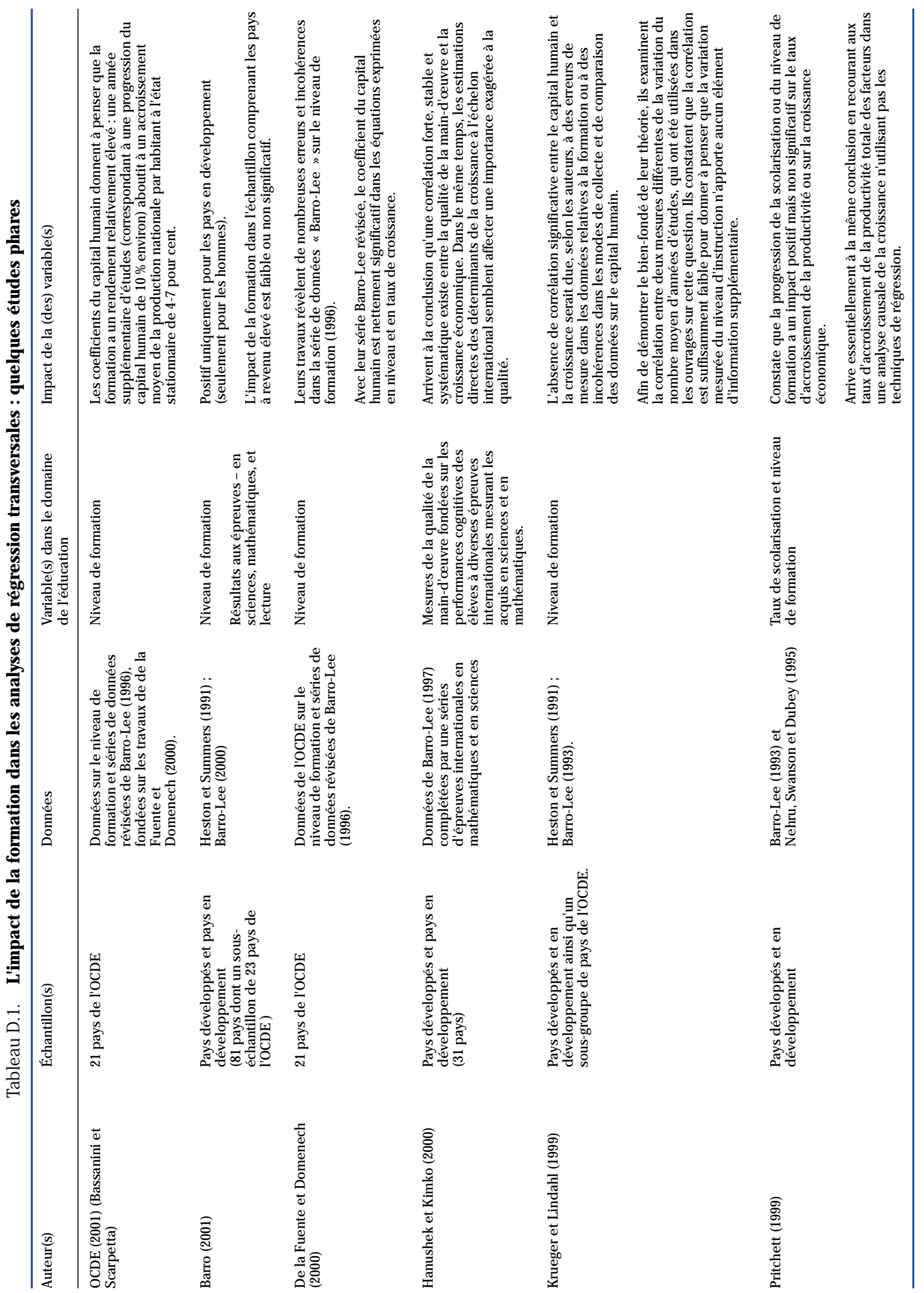




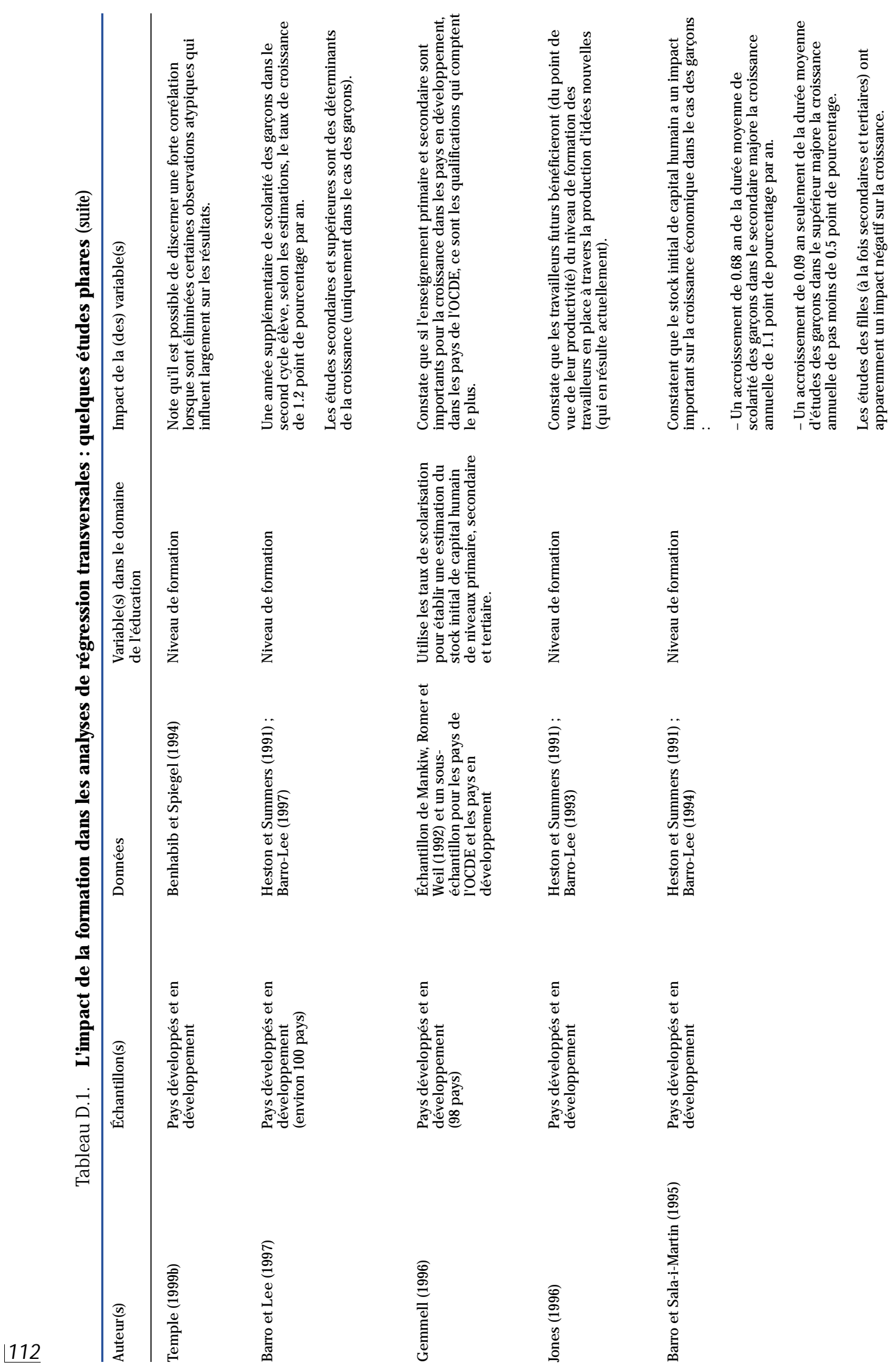




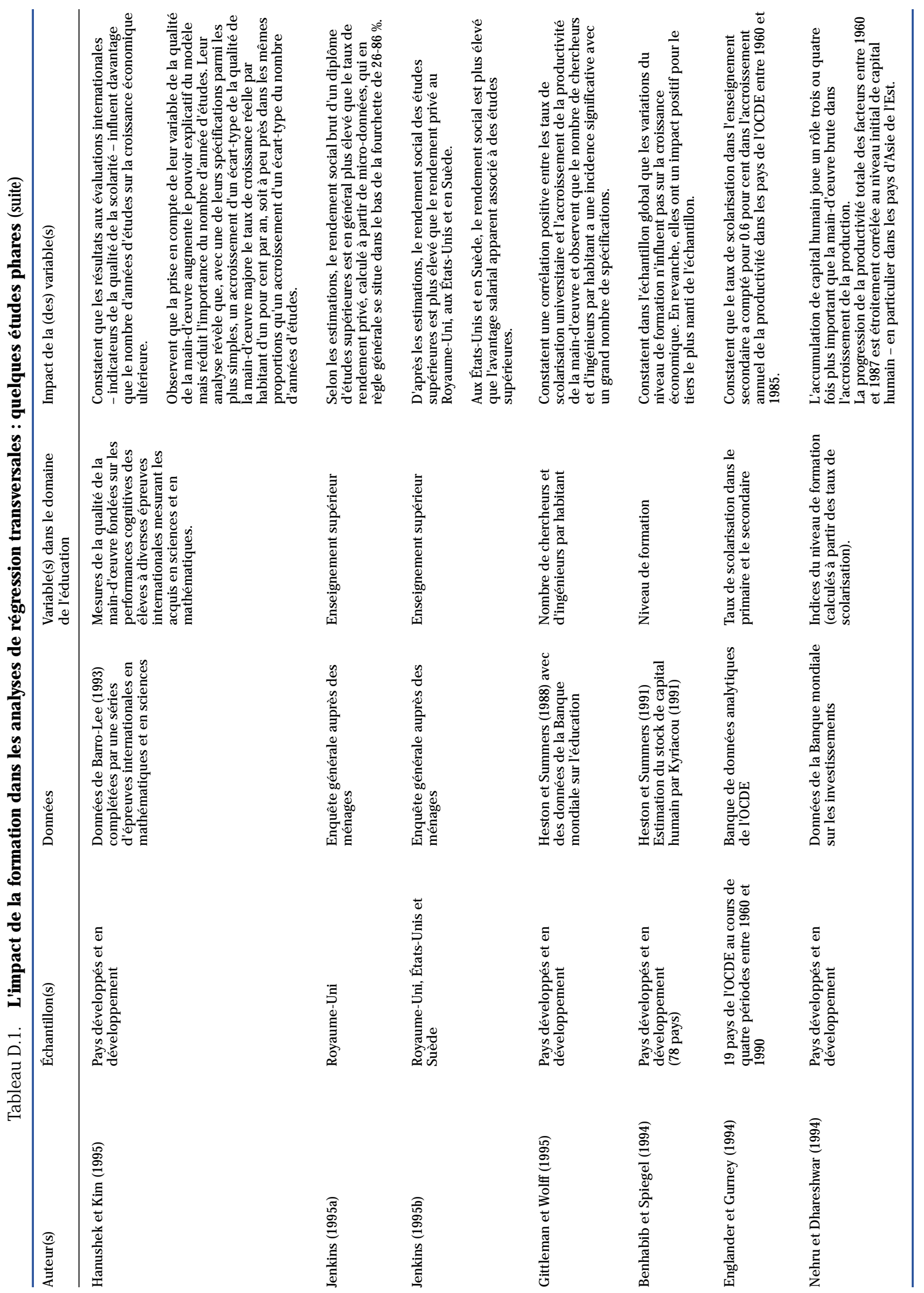




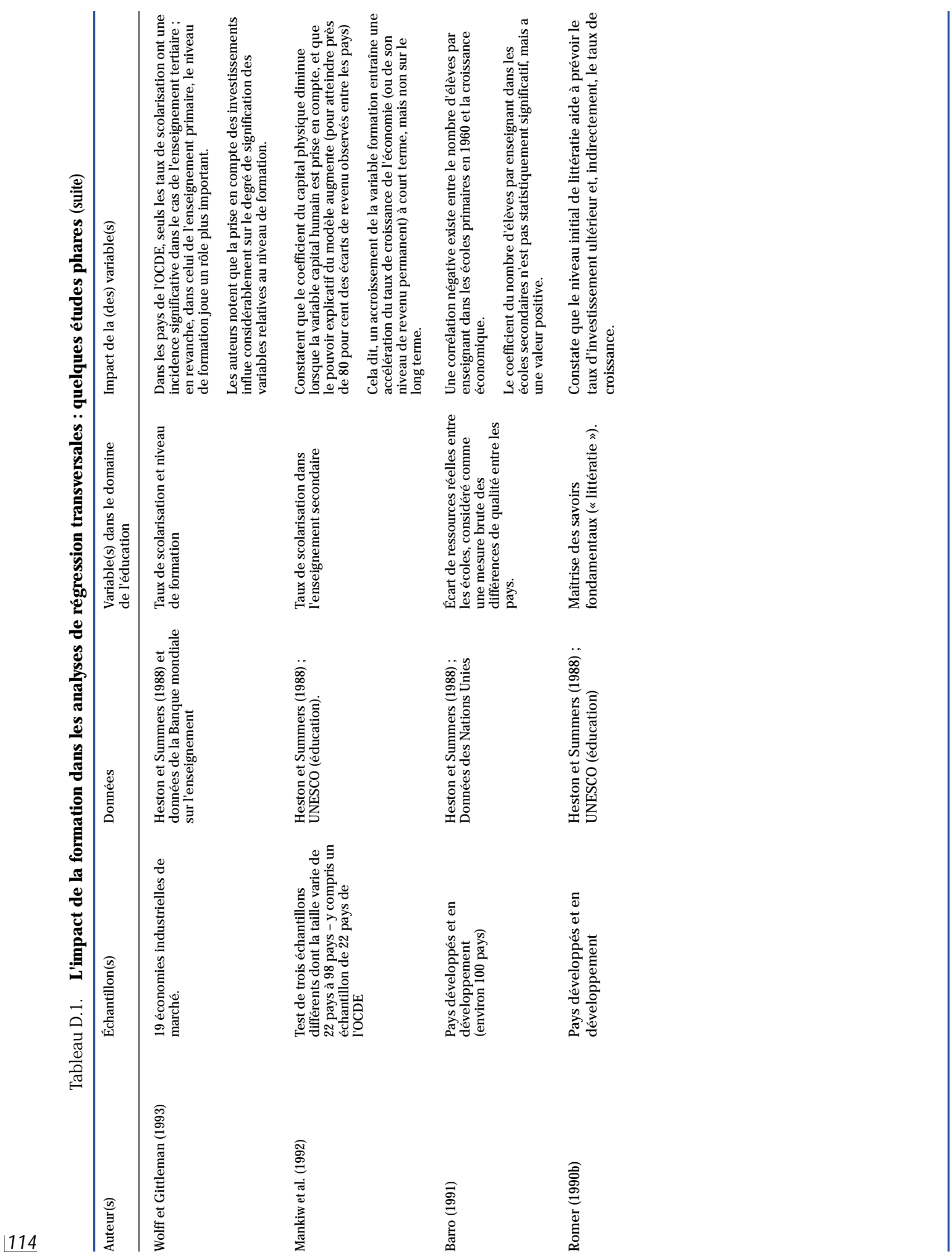


Annexe $E$

\section{LA CONFIANCE ET L'ENGAGEMENT CIVIQUE SONT-ILS EN BAISSE DANS LES PAYS DE L'OCDE?}

Cette annexe comprend des éléments d'information sur la confiance et l'engagement civique dans huit pays : les États-Unis, le Royaume-Uni, les Pays-Bas, la Suède, I'Australie, le Japon, la France et l'Allemagne.

\section{États-Unis}

En se fondant sur des données provenant de sources diverses (dont la G eneral Social Survey des États-Unis, I'enquête sur les tendances sociales et politiques de Roper et l'enquête sur les styles de vie de DDB Needham qui fournissent d'abondantes séries de données portant sur plusieurs décennies), Putnam (2000a et 2000b) fait observer que l'adhésion à des groupes et les contacts sociaux informels diminuent aux États-Unis. Le recul des taux d'adhésion à des organisations officielles s'est accompagné d'une baisse encore plus marquée de l'intensité de la participation (nombre de réunions, volonté de jouer un rôle d'encadrement, etc.). Ce phénomène est intervenu malgré l'élévation des niveaux de formation qui est généralement associée à un accroissement de l'engagement civique. Au milieu des années 70 , près des deux tiers des Américains déclaraient avoir assisté à des réunions de club au cours des douze mois précédents (voir le graphique E.1). A la fin des années 90, près des deux tiers n'assistaient jamais à ce type de réunion*

\section{Graphique E.1. Baisse du nombre de réunions de clubs aux États-Unis, 1975-1999}

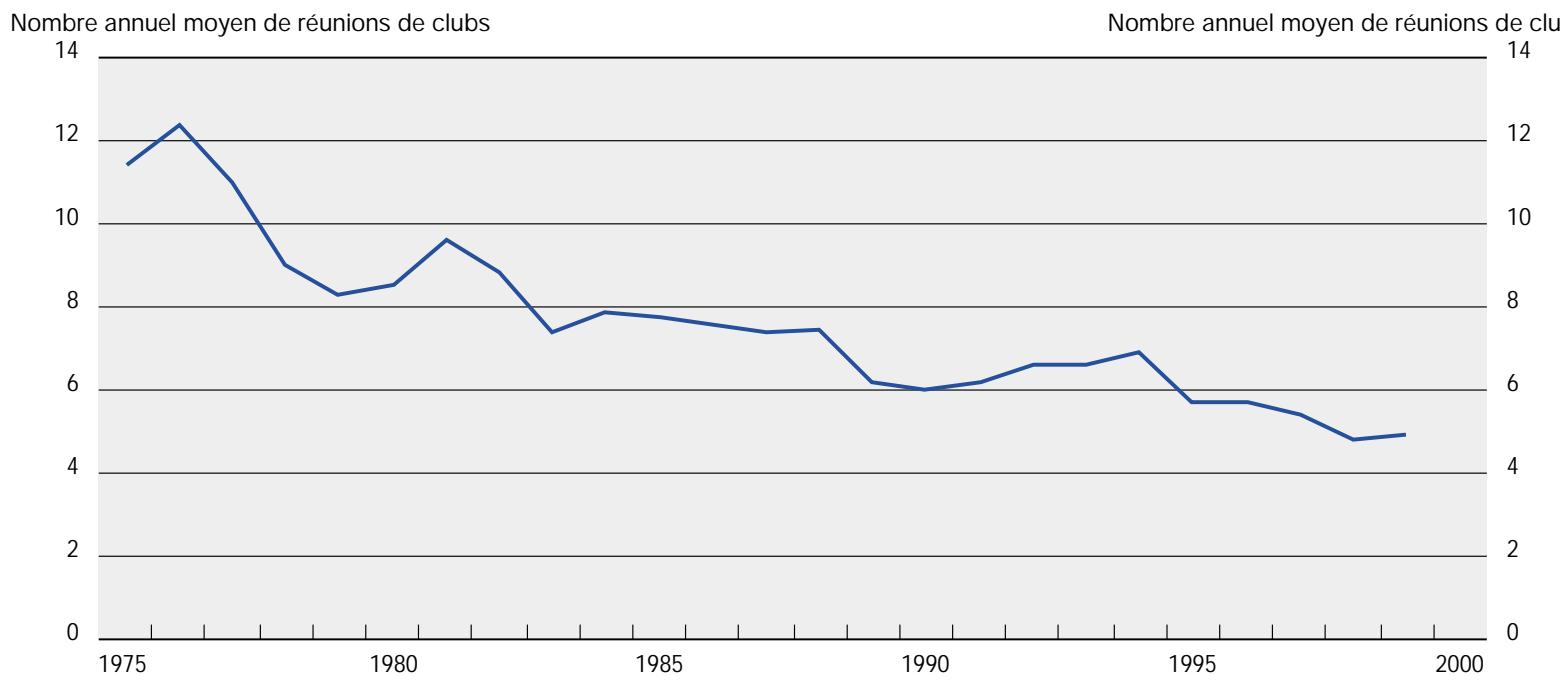

Données : Archives de l'enquête DDB Needham sur les modes de vie, 1975-99.

Source: Robert Putnam, Bowling Alone, Figure 11, p. 61 (Simon et Schuster, 2000).

*. En outre, en heures par mois, l'investissement moyen qu'un Américain consacre à la vie associative (en dehors des groupes religieux) est tombé de 3.7 heures par mois en 1965 à 2.9 en 1975, à 2.3 en 1985 et 1995. Putnam (2000a), pp. 61-62. 
L'engagement dans des organisations politiques, civiques, religieuses et professionnelles diminue depuis 25 ans aux États-Unis, qu'on le mesure en nombre absolu de membres ou en «part de marché » (pourcentage de la population visée pouvant être membre d'une organisation donnée).

Ces éléments d'information amènent à se poser un certain nombre de questions. Les changements constatés peuvent être soit temporaires, soit l'inévitable résultat de l'évolution des normes et des structures sociales. En revanche, les tendances observées pourraient induire en erreur si d'autres formes moins classiques d'organisation sociale avaient progressé, mais n'étaient pas reflétées dans les séries chronologiques. II pourrait, par exemple, y avoir un renforcement des nouveaux mouvements sociaux, notamment de ceux qui militent en faveur de l'égalité hommesfemmes, de l'environnement, des droits civils ou d'un sujet précis. Les données à ce sujet sont contrastées, mais tendent effectivement à indiquer que la «participation » est généralement moins intense et moins soutenue que dans les secteurs plus traditionnels d'association civique. Le nombre de leurs membres est très fluctuant et dans bien des cas est calculé à partir des « listes de publipostage ». Les groupes informels d'aide mutuelle et de soutien se sont certainement développés, mais l'engagement dans ces groupes paraît plus provisoire, de courte durée et moins associé à des questions d'intérêt public général.

Diverses formes de contacts sociaux informels (repas en famille, pique-niques, parties de cartes, dîners entre amis, visites, repas à l'extérieur, etc.) ont aussi diminué sur le long terme. Les gens semblent consacrer plus de temps à eux-mêmes et à leur famille proche qu'à la collectivité en général. Pour ce qui est des activités sportives, la présence à des manifestations sportives en tant que spectateur augmente (de même que les visites de musée, les sorties au cinéma, etc.) mais la participation active à un sport (ou à une activité musicale) diminue.

Le niveau général de confiance entre les personnes a également fléchi. Cette baisse est fortement liée à l'âge, les personnes nées entre 1945 et 1965 et celles qui sont nées ap rès 1965 faisant moins confiance à autrui que les générations précédentes. Au sein des cohortes, les niveaux de confiance sont stables au fil du temps. Les grands-parents font plus de deux fois plus confiance à autrui que les petits-enfants ( 50 pour cent contre 20 pour cent). Ils sont presque deux fois plus nombreux à aller voter que les cohortes plus récentes (80-85 pour cent contre 45-50 pour cent) et ils s'intéressent presque deux fois plus à la politique (55 pour cent contre 30-35 pour cent). Aux États-Unis, la baisse de la confiance, de l'intérêt pour la politique et de l'activité politique proprement dite semble s'observer essentiellement entre générations.

\section{Graphique E.2. Personnel employé à la justice et à la sécurité aux États-Unis, 1900-1997}

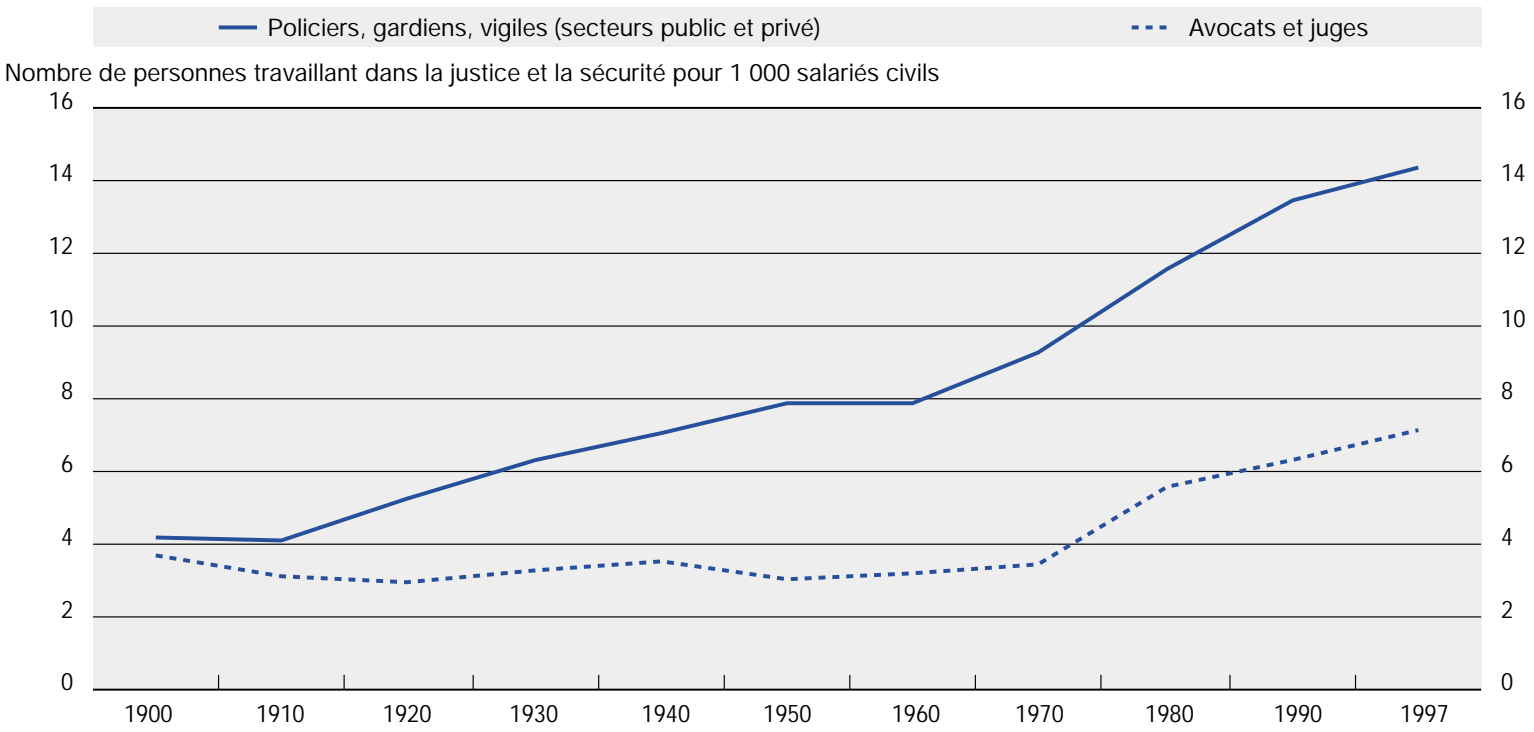

Données : 1900-70: Historical Statistics of the United States, partie I, D589 2, $144 ; 1970-96$ : Statistical Abstract of the United States et données provenant du Bureau of Labor Statistics (BLS).

116 Source: Robert Putnam, Bowling Alone, Figure 42, p. 145 (Simon et Schuster, 2000). 
Un autre indicateur possible de la baisse du niveau de confiance ou de loyauté est l'accroissement du nombre d'avocats depuis 1970, qui selon Putnam serait lié à ce qu'il appelle la «prévention du contentieux ». Selon cet auteur, la stabilité du nombre d'avocats jusqu'en 1970, malgré un bouleversement social et économique profond, est révélateur des changements particuliers qui sont survenus depuis la fin des années 60 (voir le graphique E.2).

Les taux de participation à des activités bénévoles, collectives, sociales et caritatives sont en général plus élevés parmi les personnes d'âge mûr et relativement âgées que parmi les jeunes. Altruisme, bénévolat et philanthropie ont une forte corrélation entre eux. Le don de temps et d'argent est lié à l'engagement dans la vie collective plus qu'à l'instruction, à la richesse, à la taille de la collectivité, à la situation de famille ou à l'emploi. Malgré la réd uction de l'engagement civique dans d'autres domaines, le bénévolat a progressé aux États-Unis essentiellement dans les cohortes âgées (que Putnam appelle la « longue génération civique » des personnes nées entre 1910 et 1940). Les activités bénévoles de par leur nature s'orientent plutôt vers les soins aux personnes que vers des activités de groupe de type collectif.

\section{Royaume-Uni}

Hall (1999) a découvert que les adhésions à des associations ont augmenté dans la plupart des cas depuis les années 50. Comme les États-Unis, le Royaume-Uni a une longue tradition de culture civique assortie de hauts niveaux de confiance sociale et de participation politique et civique (Almond et Verba, 1963). Contrairement à ce qui se passe aux États-Unis, Hall ne constate aucune réduction de l'engagement dans le temps, même chez les jeunes (ce qui contredit I'hypothèse selon laquelle des changements entre générations seraient en cours, mais n'ap paraîtraient pas encore dans les données démographiques globales). II note une baisse de la participation à des organisations traditionnelles féminines ou religieuses, mais ne dispose pas de données qui permettraient d'étudier l'intensité de la participation ou la qualité de l'engagement à long terme. II est possible que la participation dans les organisations de défense des intérêts publics ait diminué au profit de l'adhésion à des organisations d'entraide ou s'occupant de problèmes spécifiques. En outre, la participation directe semble diminuer au profit de formes d'engagement moins formelles et moins soutenues. Les données ne permettent pas d'analyser ces éventualités.

Selon les observations de Hall, les taux d'adhésion à des organisations et les niveaux de confiance déclarés révèlent toutefois un écart croissant entre divers groupes sociaux, notamment en fonction du niveau d'instruction. La confiance et l'engagement ont une corrélation positive avec le niveau d'instruction et sont en général plus développés dans la classe moyenne que dans la classe ouvrière. Hall constate aussi que l'importance relative des divers groupes a changé, la classe ouvrière et les personnes ayant un faible niveau d'instruction étant proportionnellement moins représentées que dans les années 50 . II laisse entend re que la réforme et la démocratisation de l'enseignement, conjuguées à une évolution de la structure des classes sociales, a pu renforcer les niveaux de confiance et d'engagement civique au Royaume-Uni, préservant les niveaux globaux de capital social. Le soutien de l'État en faveur du bénévolat (aide sociale, prise en charge des personnes âgées, projets d'intérêt collectif, etc.) a peut-être également joué un rôle important. Les taux de participation des femmes à la vie collective ont augmenté. Hall recense trois raisons pour expliquer ce phénomène : le pourcentage plus élevé de femmes qui font des études supérieures, l'activité féminine croissante et les changements généraux de la situation sociale des femmes. En particulier, la participation à la vie active des hommes et des femmes ayant fait des études supérieures a augmenté davantage que celle des autres personnes (bien qu'elle ait augmenté à tous les niveaux d'instruction). Les études supérieures pourraient donc avoir sur le capital social un effet marginal plus élevé qu'il ne l'était autrefois.

Les données d'enquête sur l'utilisation du temps couvrant la période 1961 à 1984 ne donnent aucune ind ication probante d'une privatisation croissante des loisirs ou d'une réduction des contacts sociaux informels (bien que le temps passé à rendre visite à des amis ait diminué, surtout pour les hommes travaillant à plein temps). Cela dit, les niveaux de confiance entre les personnes ont diminué. En 1959, 56 pour cent des personnes répondant à l'enquête sur la culture civique avaient déclaré faire confiance à autrui alors qu'en 1995, le chiffre correspondant était tombé à 31 pour cent (World Values Study pour le Royaume-Uni). Hall signale aussi la possibilité qu'avec le temps les valeurs et les attitudes aient subi des modifications importantes qui influent sur la qualité de l'engagement civique et de la confiance. Ce phénomène semble s'inscrire dans la tendance plus générale à l'adoption de valeurs plus matérialistes et individualistes notée dans les chapitres 1 et 3. Putnam (2000b) note aussi une évolution importante des valeurs et des 
attitudes des jeunes par rapport à celles du même groupe d'âge, il y a 20 ou 30 ans. C'est davantage parmi les jeunes que d'autres groupes d'âge que les niveaux de confiance baissent. Les données relatives au Royaume-Uni indiquent une diminution de la confiance :

- entre personnes (dénommée ici confiance interpersonnelle générale);

- à l'égard des institutions et des pouvoirs publics.

Globalement, le Royaume-Uni semble se caractériser par un accroissement de la vie associative et des contacts sociaux informels, conjugué à une baisse des niveaux de confiance. Le développement général de la vie associative dissimule un écart croissant, depuis les années 50 , entre les « groupes de citoyens très actifs et ayant de nombreuses relations » et les autres qui n'ont qu'une vie associative et un engagement civique très limités. Les deux groupes les plus touchés sont les jeunes et les membres de la classe ouvrière. II faut aussi noter que, toutes choses étant égales d'ailleurs, les personnes qui ont divorcé, connu le chômage ou déménagé vers une grande ville sont moins disposées à participer à la vie associative ou à faire confiance aux autres.

\section{Pays-Bas}

Les données relatives aux Pays-Bas n'indiquent pas de baisse globale du capital social, mais laissent entrevoir une évolution possible des types d'engagement. De Hart et Dekker (1999) examinent les données relatives aux adhésions à divers types d'organisations et aux taux de bénévolat. Le nombre d'ad hésions à des associations et l'intensité de l'engagement semblent augmenter pour la plupart des types d'organisation (sauf pour les associations féminines traditionnelles et les partis politiques). Entre 1980 et 1985, les activités bénévoles ont été stables ou en progrès bien qu'elles aient diminué pour le groupe d'âge de 18 à 34 ans (enquête néerlandaise sur l'utilisation du temps). L'activisme politique a eu tendance à augmenter et le taux de participation des électeurs aux grands scrutins est resté stable. Toutefois, comme dans d'autres pays européens, la participation semble prendre des formes moins intenses et moins engagées. Ce sont les mouvements sociaux défendant une seule cause - l'environnement, la solidarité internationale ou des problèmes moraux - qui sont en progression rapide. S'il est difficile d'évaluer l'ampleur de la participation active à ces types d'organisations, il est probable que l'engagement y est plus provisoire et moins orienté vers des questions d'intérêt public général que dans d'autres types d'organisations civiques. Enfin, les données sur l'utilisation du temps ne donnent pas à penser que les niveaux d'engagement ou les contacts sociaux informels aient diminué.

\section{Suède}

Rothstein (1998) passe en revue des données révélant une détérioration de ce qu'il appelle «le capital social organisé ", c'est-à-dire de la confiance existant au sein des grandes organisations syndicales et patronales et entre elles pendant une période de consensus soutenu et de participation des partenaires sociaux à la planification économique et sociale. Comme pour la Grande-Bretagne, il signale une diminution de la confiance dans les institutions politiques parallèle à une baisse des niveaux de coopération, de consensus et de confiance depuis la fin des années 80. L'engagement politique semble aussi évoluer d'un engagement actifà une attitude et à un intérêt plus passifs de spectateur. S'il ressort des données que la population s'intéresse de plus en plus à la politique, le nombre de participants actifs diminue : les bénévoles sont plus âgés et, par ailleurs, la professionnalisation des hommes politiques ainsi que la participation plus grande des médias ont en général eu pour effet d'atténuer le rôle de ces bénévoles. Les campagnes professionnelles et l'activité médiatique ont tendance à supplanter la mobilisation populaire, le déb at et les cercles d'étude. Comme dans d'autres pays européens, les adhésions à des groupes s'occupant d'une question précise progressent par rapport au nombre de membres d'organisations de masse plus stables.

Traditionnellement, la Suède, comme les autres pays scandinaves, se place en très bon rang dans les classements internationaux concernant l'engagement dans la vie associative, le bénévolat et les niveaux déclarés de confiance générale. II ressort d'enquêtes nationales et internationales telles que la World Values Study que les organisations sociales, officielles ou non, ont plutôt gagné en vitalité ces dernière décennies. Rothstein signale des indices d'individualisme 
croissant, à mesure que les jeunes générations se détournent des formes d'activités sociales traditionnelles organisées sur une base hiérarchique. Ainsi, les mouvements religieux de lutte contre l'alcoolisme et les organisations féminines ont fait place à des organisations sportives, culturelles, écologiques et de loisirs. Comme au Royaume-Uni, les taux d'adhésion à des organisations varient selon les groupes et c'est parmi les jeunes qu'ils sont le plus élevés. Ces taux d'adhésion ont progressé avec le temps pour tous les groupes d'âge et toutes les classes sociales et l'écart entre les hommes et les femmes a diminué (comme au Royaume-Uni). La World Values Study indique aussi une augmentation entre 1981 et 1996. Les enquêtes suédoises sur les niveaux de vie de 1968, 1981 et 1991 vont dans le même sens.

D'autres études révèlent toutefois une diminution des niveaux d'« affinité » avec certaines organisations de masse. Selon Rothstein, cela est peut-être dû à un discernement accru ou à un affaiblissement de l'identité collective ou de groupe. D'autres études encore indiquent une montée de l'individualisme et de l'autonomie personnelle. Rothstein soutient que cette attitude peut être compatible avec un individualisme plus solidaire (par opposition à l'individualisme égoïste).

\section{Australie}

Les données sur l'Australie ressemblent davantage à celles sur les États-Unis. Eva Fox (dans Putnam, 2000b) a noté que les ad hésions à de nombreux groupes bénévoles établis de longue date ont diminué au cours des dernières décennies. Par exemple, le nombre de salariés syndiqués et le nombre de personnes allant à l'église ont sensiblement baissé entre les années 60 et les années 90 . Le bénévolat semble aussi avoir diminué ; quant aux chiffres sur l'engagement politique, ils ne sont pas explicites. La confiance générale entre les personnes et la confiance dans les institutions politiques ont nettement fléchi au cours des 15 dernières années. On regarde davantage la télévision et on voit moins ses amis. Selon Cox, la baisse de confiance est peut-être liée à une anxiété croissante quant à l'avenir ainsi qu'à une montée des inégalités. Si les activités sportives sont en hausse, comme elles semblent l'être ailleurs, il s'agit le plus souvent d'activités individuelles de mise en forme plutôt que de sports d'équipe. Certains mouvements sociaux comme les groupes féministes ou écologiques semblent avoir perdu du terrain.

\section{Japon}

Inoguchi (dans Putnam, 2000b) observe une hausse des niveaux d'engagement civique et d'adhésion à des organisations non gouvernementales au Japon. L'appartenance à des groupes de quartier n'a pas sensiblement changé depuis le milieu des années 80 . La participation à des organisations bénévoles caritatives s'occupant d'enfants, de personnes âgées et de personnes défavorisées a fortement progressé.

La confiance sociale a peut-être un rayon d'action plus court au Japon qu'en Europe du Nord et aux États-Unis. Inoguchi soutient que la confiance et la coopération sont plus fortes dans les cercles petits et intimes de la famille, des amis proches et des collègues. De l'avis de Fukuyama (1995), dans la société japonaise, la confiance se caractérise par la transformation d'entreprises familiales en de vastes sociétés grâce à une " société riche et complexe » (p. 130). L'industrie manufacturière japonaise, économe en ressources, est présentée comme le modèle du « lieu de travail où le niveau de confiance est élevé » et où le rôle des travailleurs est de donner leur avis pour aider à gérer la chaîne de production dans son ensemble (pp. 258-259). On semble assister à une augmentation progressive de la confiance généralisée, ce qui donne à penser que le Japon pourrait converger vers les schémas établis ailleurs. Peu de données existent au sujet du niveau de socialisation informelle.

\section{France}

Les données analysées par Worms dans Putnam (2000b) ne révèlent aucune baisse de la vie associative. Si certaines des grandes organisations sociales et politiques, surtout les syndicats, les partis politiques et l'église, ont moins de membres, les adhésions à d'autres types d'associations sont stables. Il se peut que les politiques et prestations sociales aient soutenu des organisations de services sociaux à but non lucratif. Deux tendances sont à noter 
au cours des dernières décennies. D'abord, on a observé une montée des organisations s'occupant d'intérêts sectoriels ou particuliers, suivie par une augmentation des adhésions à des organisations ayant des intérêts plus généraux. Ensuite, on a enregistré une progression des associations de dévelop pement personnel sous forme d'activités culturelles et de loisirs. Ces changements seraient partiellement attribuables à l'éducation.

Comme dans d'autres pays, la participation civique semble s'être orientée vers des affiliations plus informelles et temporaires. Worms avance l'idée que la nature changeante de l'engagement évoque un « maillon manquant » entre la sociabilité privée et l'intérêt public au sens plus large, surtout pour ce qui est des liens avec les institutions publiques. II y a toutefois une différence notable d'engagement civique entre la France et d'autres pays. La France se place derrière les États-Unis et les pays scandinaves, mais aussi derrière l'Allemagne, la Belgique, le RoyaumeUni, I'Irlande pour ce qui est des adhésions aux organisations, du nombre de bénévoles et du nombre de donneurs. Selon Worms, il est possible que la concurrence que l'Église et l'État se sont livré dans le passé pour contrôler la société civile ait laissé peu de marge à l'initiative des citoyens.

\section{Allemagne}

La participation à des activités sociales officielles et informelles, qui était très faible en 1945, a progressé depuis en Allemagne (Offe et Fuchs, 1998), et surtout chez les jeunes. Quelques exceptions sont à noter : 1) les syndicats, les partis politiques et les égl ises ont perdu des membres; et 2) les jeunes Allemands semblent s'être détournés des organisations politiques et sociales dans les années 90 . Comme en Suède, la tendance est à l'ab andon des organisations officielles au profit d'une participation plus temporaire et individualiste. La répartition des activités sociales entre les divers groupes est assez proche de celle des États-Unis : la participation à des associations (surtout officielles) progresse chez les personnes instruites et aisées mais aussi parmi les actifs, les personnes d'âge mûr, dans les petites villes et chez les hommes (surtout dans les formes «publiques » d'activités, encore que l'écart entre hommes et femmes diminue au fil des ans). 


\section{BIBLIOGRAPHIE}

ABRAM OVITZ, M. et DAVID, P. (1996), «Convergence and Deferred Catch-up : Productivity Leadership and the Waning of American Exceptionalism », dans R. Landau, T. Taylor et G. Wright (dir. pub.), The M osaic of E conomic G rowth, Stanford University Press, Standford, CA.

ABRAMS, P. et BULMER, M. (1986), N eighbours, Cambridge University Press, Cambridge, Royaume-Uni.

ACEMOGLU, D. (1996),

«A Microfoundation for Social Increasing Returns in Human Capital Accumulation », Q uarterly Journal of Economics, vol. 111, pp. 779-804.

ADLER, P. et KWON, S. (2000), «Social Capital : The Good, the Bad, and the Ugly », University of Southern California.

AGHION, P. et HOWITT, P. (1998), Endogenous $G$ rowth Theory, MIT Press, Cambridge, MA.

ALESINA, A. et RODRIK, D. (1992), «Income Distribution and Economic Growth : A Simple Theory and Empirical Evidence », dans A. Cukierman, Z. Hercowitz et L. Leiderman (dir. pub.), The Political E conomy of Business Cycles and G rowth, MIT Press, Cambridge, MA.

ALMOND, G. et VERBA, S. (1963),

The Civic Culture : Political Attitudes and D emocracy in Five N ations, Princeton University Press, Princeton.

AMATO, P. (1998), «More than Money? Men's Contributions to their Children's Lives », dans A. Booth et A. Creuter (dir. pub.), M en in F amilies: When do they get involved? What difference does it make?, Lawrence Erlbaum, New Jersey, chapitre 13.

ANGRIST, J. et KRUEGER, A.B. (1991a), « Does Compulsory School Attendance Affect Schooling and Earnings? », Quarterly Journal of Economics, vol. 106, pp. 979-1014.

ANGRIST, J. et KRUEGER, A.B. (1991b), «Estimating the Payoff to Schooling Using the Vietnam-Era Lottery », Princeton University Industrial Relations Section, Document de travail $n^{\circ} 290$, août.

ARROW, K.J. (1972),

«Gifts and Exchanges, » Philosophy and Public Affairs, $n^{\circ} 1$, été.

ASHENFELTER, O. et KRUEGER, A.B. (1994), «Estimates of the Economic Return to Schooling from a New Sample of Twins », American E conomic Review, vol. 84, $n^{\circ}$ 5, décembre, pp. 1157-1173.

AXELROD, R. (1984), The E volution of Cooperation, Penguin, New York.

BAKER, B. et BENJAMIN, D. (1994), «The Performance of Immigrants in the Canadian Labor Market », Journal of Labour E conomics, vol. 12, $n^{\circ} 3$.

BANQUE MONDIALE (1998), "The Initiative on Defining, Monitoring and Measuring Social Capital Text of Proposals Approved for Funding », Environmentally and Socially Sustainable Development Network, Social Capital Initiative, Working Paper No. 2, juin.

BARBIERI, P., RUSSELL, H. et PAUGAM, S. (1999),

«Social Capital and Exits from Unemployment », rapport non publié. 
BARRO, R.J. (1991),

«Economic Growth in a Cross-section of Countries », Quarterly Journal of E conomics, CVI, mai.

BARRO, R.J. (2001),

«Education and Economic Growth », dans J.F. Helliwell (dir. pub.), The Contribution of H uman and Social Capital to Sustained $\mathrm{E}$ conomic $\mathrm{G}$ rowth and Well-being : International Symposium Report, Développement des ressources humaines Canada et OCDE.

BARRO, R.J. et LEE, J.W. (1993),

«International Comparisons of Educational Attainment », NBER Working Paper No. 4349.

BARRO, R.J. et LEE, J.W. (1994),

«Data Set for a Panel of 138 Countries », révisé, janvier.

BARRO, R.J. et LEE, J.W. (1996),

"International Measures of Schooling Years and Schooling Quality », American Economic Review, Papers and Proceedings $86, n^{\circ} 2, p p .218-223$.

BARRO, R.J. et LEE, J.W. (1997),

«Schooling Quality in a Cross-section of Countries », NBER Working Paper No. 6198.

BARRO, R.J. et LEE, J.W. (2000),

«International Data on Educational Attainment: Updates and Implications », Center for International Development (CID), Working Paper No. 42, Harvard University, avril.

BARRO, R.J. et SALA-I-MARTIN, X. (1995), E conomic G rowth, McGraw-Hill, New York.

BASSANINI, A. et SCARPETTA, S. (2001),

«Liens entre la politique économique et la croissance : constatations au niveau international », Département des Affaires économiques de l'OCDE.

BECKER, G. (1993),

«Human Capital : A theoretical and Empirical Analysis, with Special Reference to Education », The University of Chicago Press, Chicago, troisième édition.

BEHRMAN, J.R. et STACEY, N. (dir. pub.) (1997),

The Social B enefits of Education, The University of Michigan Press, Ann Arbor.

BENHABIB, J. et SPIEGEL, M. (1994),

"The Role of Human Capital in Economic Development : Evidence from Aggregate Cross-Country Data », Journal of M onetary E conomics, vol. 43, pp. 143-174.

BETTS, J. (1995),

« Does School Quality Matter? Evidence from the National Longitudinal Survey of Youth », Review of Economics and Statistics, mai, n 77(2), pp. 231-250.

BETTS, J. et ROEMER, J. (1998),

«Equalizing Opportunity through Educational Finance Reform », Department of Economics, University of California, San Diego.

BIBLARZ, T., RAFTERY, A. et BUCUR, A. (1997), «Family Structure and Social Mobility », Social Forces, vol. 75(4), pp. 1319-1339.

BISHOP, J. (1999),

«Are National Exit Examinations Important for Educational Efficiency? », Swedish E conomic Policy Review, vol. 6(2), pp. 349-398.

BLANCHFLOWER, D.G. et OSWALD, A.J. (2000), «Well-being over Time in Britain and the USA », Working Paper No. 7487, National Bureau of Economic Research, Cambridge, MA (http://www.nber.org/papers/w7487).

BLOSSFELD, H.P. et SHAVIT, Y. (1993),

Persistent Inequality : Changing Educational Attainment in Thirteen Countries, Westview Press Inc, Colorado.

$\overline{\text { C) OCDE } 2001}$ 
BOOTHBY, D. (1999),

«Literacy Skills, the Knowledge Content of Occupations and Occupational Mismatch », Document de recherche de la Direction générale de la recherche appliquée, Développement des ressources humaines Canada, août.

BORJAS, G.J. (1998),

«The Economic Progress of Immigrants », NBER Working Paper No. 6506.

BOURDIEU, P. (1979),

«Les trois états du capital culturel », Actes de la recherche en sciences sociales, n 30 (« L'institution scolaire »), pp. S.3-6.

BOURDIEU, P. (1980),

«Le capital social : notes provisoires », Actes de la recherche en sciences sociales I, n 31, pp. 2-3.

BOURDIEU, P. (1985),

«The Forms of Capital », dans J.E. Richardson (dir. pub.), H andbook of Theory of Research for the Sociology of E ducation, Greenwood Press, New York, pp. 241-258.

BOURDIEU, P. et PASSERON, J.C. (1970), Reproduction in Education, Society and Culture, Sage, Londres.

BRINK, S. et ZEESMAN, A. (1997), « Mesure du bien-être social : Un indice de santé sociale pour le Canada », Document de travail de DRHC, R-97-9F.

BROWN, G. et HARRIS, T. (1978), Social $O$ rigins of D epression, Tavisock, Londres.

BRYK, A.S., LEE, V.E. et HOLLAND, P.B. (1993), Catholic Schools and the Common Good, Harvard University Press, Cambridge, MA.

BURT, R.S. (1992), Structural H oles, The Social Structure of Competition, Harvard University Press, Camb ridge, MA.

BYNNER, J., MCINTOSH, S., VIGNOLES, A., DEARDEN, L., REED, H. et van REENEN, J. (2001), Wider Benefits of Learning Improving Adult Basic Skills : B enefits to the Individual and to Society, rapport établi pour le Department for Education and Employment (UK), DfEE Wider Benefits of Learning Research Centre, Institute of Education, London University, Centre for Economic Performance, London School of Economics et le Institute for Fiscal Studies.

BYNNER, J. et EGERTON, M. (à paraître),

The Wider Benefits of Higher Education, rapport parrainé par le Higher Education Funding Council for England en association avec le Smith Institute, Department for Education and Employment (UK), DfEE Wider Benefits of Learning Research Centre, Institute of Education, London University.

CAPPELLI, P. et ROGOVSKI, N. (1994),

«New Work Systems and Skills Requirements », International Labour Review, n², pp. 205-220.

CARD, D. (1994),

«Earnings, Schooling, and Ability Revisited », NBER Working Paper No. 4832, août.

CARD, D. (1999),

«Causal Effect of Education on Earnings », dans O. Ashenfelter et D. Card (dir. pub.), H andbook of Labor E conomics, vol. 3A, chapitre 30, North-Holland, Amsterdam, pp. 1801-1863.

CARLINER, G. (1996),

«The Wages and Language Skills of U.S. Immigrants », NBER Working Paper No. 5793, National Bureau of Economic Research, Cambridge, MA.

CARLSON, M. (1999),

«Do Fathers Really Matter? Father Involvement and Socio-psychological Outcomes for Adolescents », Bendheim-

Thoman Center for Research on Child Wellbeing, Working Paper 99-04, Princeton University.

COBB, C., HALSTEAD, T. et ROWE, J. (1996),

«If the GDP is Up, Why is America Down? », The Atlantic M onthly, octobre, pp. 59-78. 
COHEN, P., STRUENING, E., MUHLIN, G., GENEVIE, E., KAPLAN, S. et PECK, H. (1982), «Community Stressors, Mediating Conditions and Wellbeing in Urban Neighborhoods », Journal of Community Psychology, $n^{\circ} 10$.

COLEMAN, J. (1988), «Social Capital in the Creation of Human Capital », American Journal of Sociology, vol. 94, supplément, pp. S95-120.

COLEMAN, J.S. (1990), The Foundations of Social Theory, Harvard University Press, Cambridge.

COLEMAN, J. et HOFFER, T. (1987), Public and Private High Schools: The Impact of Communities, Basic Books, New York, pp. 94, 133-135, 231, 229 [Stephen L. Morgan et Aage B. Sørensen (1999) défendent des thèses inverses dans «A Test of Coleman's Social Capital Explanation of School Effects », American Sociological Review, $n^{\circ}$ 64, pp. 661-681].

COLEMAN, J., CAM PBELL, E., HOBSON, C., MCPARTLAND, J, MOOD, A., WEINFALL, F. et YORK, R. (1966), Equality of E ducational O pportunity, Government Printing Office, Washington, États-Unis.

COLLIER, P. (1998),

«Social Capital and Poverty », Document de travail, Banque mondiale, Washington, DC.

COUNCIL OF ECONOMIC ADVISERS (1999),

«Families and the Labor Market, 1969-1999: Analyzing the 'time crunch' », rapport du CEA, Washington, DC., mai.

COX, E. (2000), dans R. Putnam, R. (dir. pub.), Society and Civic Spirit (G esellschaft und Gemeinsinn), Bertelsmann Foundation.

COX, E. et MACDONALD, D. (2000), «Making Social Capital a Discussion Paper », New South Wales Council of Social Service.

DARLING, N. et STEINBERG, L. (1997), « Community Influences on Adolescent Achievement and Deviance, » dans Brooks-Gunn, Duncan et Aber (dir. pub.), N eighborhood Poverty, vol. II, pp. 120-131.

De HART, J. et DEKKER, P. (1999), "Civic Engagement and Volunteering in the Netherlands : A 'Putnamian' Analysis », dans J. Van Deth, M. Maraffi, K. Newton et P. Whiteley (dir. pub.), Social Capital and European Democracy, Routledge, Lond res, pp. 75-107.

De la FUENTE, A. et DOMENECH, R. (2000), «Human Capital in Growth Regressions : How Much Difference does Data Quality Make? », CSIC, Campus de la Universidad Autonoma de Barcelona.

DÉVELOPPEMENT DES RESSOURCES HUMAINES CANADA (1999), "The Social Context of Productivity : Challenges for Policy Makers», Notes de J. Lahey pour un discours au Queen's International Institute on Social Policy, 25 août.

DOBELL, R. (2001), «Social Capital and Social Learning in a Full World », dans J.F. Helliwell (dir. pub.), The Contribution of Human and Social Capital to Sustained E conomic G rowth and Well-being : International Symposium Report, Développement des ressources humaines Canada et OCDE.

DOUGHERTY, C. et JORGENSEN, D.W. (1996), «International Comparisons of the Sources of Growth », American E conomic Review, mai, pp. 25-29.

DRUCKER, P. (1993),

The Post Capitalist Society, Butterworth-Heinemann, Oxford.

DURKHEIM, E. (1893), The D ivision of Labor in Society, The Free Press, New York, 1984.

DURKHEIM, E. (1970), Suicide : A Study of Sociology, Routledge and Kegan Paul Ltd, Londres.

EASTERLY, W. et LEVINE, R. (1997), «Africa's Growth Tragedy : Policies and Ethnic Divisions », Quarterly Journal of Economics, vol. 112(4), novembre, 124 pp. 1203-1250. 
ECKERSLEY, R. (1998),

M easuring P rogress, Is Life G etting B etter?, Commonwealth Scientific and Industrial Research Organisation Publishing, Victoria, Australie.

ELIASSON, G. (2001),

«The Role of Knowledge in Economic Growth », dans J.F. Helliwell (dir. pub.), The Contribution of H uman and Social Capital to Sustained E conomic Growth and Well-being : International Symposium Report, Développement des ressources humaines Canada et OCDE.

ENGLANDER, A.S. et GURNEY, A. (1994),

«Medium-term Determinants of OECD Productivity », Études économiques de l'O CDE, n²2, OCDE, Paris.

EPSTEIN, J.L. (1995),

«School/Family/Community Partnerships : Caring for the Children we Share », Phi Delta Kappan, vol. 76, pp. 701-712.

ERIKSON, R. et JONSSON, J. (1996),

«Explaining Class Inequality in Education : the Swedish Test Case », dans R. Erikson et J.O. Jonsson (dir. pub.), Can Education Be Equalized?, Westview Press, Boulder, CO.

FIELD, J. et SPENCE, L. (2000),

«Informal learning and social capital », dans F. Coffield (dir. pub.), The N ecessity of Informal Learning, Policy Press, Bristol.

FRATIGLIONI, L., WANG, H., ERICSSON, K., MAYTAN, M. et WINBLAD, B. (2000),

«Influence of Social Network on Occurrence of Dementia : A Community-based Longitudinal Study », The Lancet, vol. 355, n 9212, 15 avril.

FREEMAN, R.B., KLEINER, M.M. et OSTROGOFF, C. (1997),

«The Anatomy and Effects of Employee Involvement », rapport présenté à la réunion de la American Economic Association.

FULLER, B. et CLARKE, P. (1994),

«Raising School Effects while Ignoring Culture? Local Conditions and the Influence of Classroom Tools, Rules and Pedagogy », Review of Educational Research, printemps, vol. 64, n² 1, pp. 119-157.

FULLER, B. et HEYNEMAN, S. (1989),

«Third World School Quality. Current Collapse, Future Potential », Educational Researcher, vol. 18(2), pp. 12-19.

FUKUYAMA, F. (1995),

Trust : The Social Virtues and the Creation of Prosperity, The Free Press, N ew York.

FUKUYAMA, F. (1999),

The G reat D isruption : Human N ature and the R econstitution of Social Order, The Free Press, New York.

GALLAND, O. (1999),

«Les Relations de Confiance », La Revue Tocqueville, The Tocqueville Review, vol. XX, $n^{\circ} 1$.

GALLIE, D., GERSHUNY, J. et VOGLER, C. (1994), «Unemployment, the Household and Social Networks », dans Gallie et al. (dir. pub.), Social Change and the Experience of Unemployment, Oxford University Press.

GARBARIN O, J. et SHERMAN, D. (1980),

«High-Risk Neighborhoods and High-Risk Families : The Human Ecology of Child Maltreatment », Child D evelopment, $n^{\circ} 51$, pp. 188-198.

GASKIN, K. et DAVIS SMITH, J.D. (1995),

A N ew Civic Europe? A Study of the Extent and Role of Volunteering, The Volunteer Center, Londres.

GEERTZ, C. (1962),

Social Change and E conomic Modernization in Two Indonesian Towns : A Case in Point, Bobbs-Merrill, Indianapolis.

GEMMELL, N. (1995),

«Endogenous Growth, the Solow Model and Human Capital », Economics of Planning, n² 28, pp. 169-183.

GEMMELL, N. (1996),

«Evaluating the Impacts of Human Capital Stocks and Accumulation on Economic Growth : Some New Evidence », 0 xford Bulletin of E conomics and Statistics, $n^{\circ} 58, p p .9-28$. 
GEMMELL, N. (1997),

«Externalities to Higher Education : A Review of the New Growth Literature », Report 8 in the National Committee of Inquiry into Higher Education, Higher Education in the Learning Society, Reports 5-9, pp. 109-149.

GINGRAS, Y. et ROY, R. (1998), "Y-a-t-il pénurie de main-d'œuvre qualifiée au Canada? », Document de travail, recherche appliquée, Développement des ressources humaines Canada.

GITTLEMAN, M. et WOLFF, E.N. (1995), «R\&D Activity and Cross-country Growth Comparisons », Cambridge Journal of E conomics, vol. 19, pp. 189-207.

GLAESER, E.L. (2001),

"The Formation of Social Capital », dans J.F. Helliwell (dir. pub.), The Contribution of Human and Social Capital to Sustained E conomic G rowth and Well-being : International Symposium Report, Développement des ressources humaines Canada et OCDE.

GRANOVETTER, M. (1973),

«The Strength of Weak Ties », A merican Journal of Sociology, $n^{\circ} 78, \mathrm{pp} .1360-1380$.

GREEN, F., ASHTON, D., BURCHELL, B., DAVIES, B. et FELSTEAD, A. (1997), "An Analysis of Changing Work Skills in Britain », rapport présenté à la Analysis of Low Wage Employment Conference, Centre for Economic Performance, Lond on School of Economics, 12-13 décembre.

GREEN, F., MCINTOSH, S. et VIGNOLES, A. (1999), "Overeducation and Skills - Clarifying the Concepts », Centre for Economic Performance, Labour Market Programme Discussion Paper No. 435, London School of Economics.

GRILICHES, Z. (1996), «Education, Human Capital, and Growth : A Personal Perspective », NBER Working Paper No. 5426, janvier.

GROGGER, J. (1996), «Does School Quality Explain the Recent Black/White Wage Trend? », Journal of Labor E conomics, vol. 14(2), pp. 231253, avril.

GUISO, L., SAPIENZA, P. et ZINGALES, L. (2000), «The Role of Social Capital in Financial Development », NBER Working Paper No. 7563, février.

GUNDLACH, E., WOSSMANN, L. et GMELIN, J. (2000), "The Decline of Schooling Productivity in OECD Countries », rapport présenté à la réunion annuelle de la Royal Economic Society, St Andrews, 10-13 juillet.

HALL, P. (1999),

«Social Capital in Britain », British Journal of Political Science, $n^{\circ} 29$, pp. 417-461.

HALL, R. et JONES, C. (1999), «Why Do Some Countries Produce So Much More Output per Worker than Others? », Quarterly Journal of E conomics, février, vol. 114, pp. 83-116.

HALPERN, D.S. (à paraître), «Moral Values, Social Trust and Inequality : Can Values Explain Crime? », British Journal of Criminology, vol. 41(2).

HALPERN, D.S. et NAZROO, J. (2000), "The Ethnic Density Effect : Results from a National Community Survey of England and Wales », International Journal of Social Psychiatry, vol. 46 (1), pp. 34-46.

HANIFAN, L. (1916), «The Rural School Community Center », Annals of the American Academy of Political and Social Science, $n^{\circ} 67$.

HANUSHEK, E.A. (1992), «The Trade-Off between Child Quantity and Quality », Journal of Political Economy 100, $n^{\circ} 1$, février, pp. 84-117.

HANUSHEK, E.A. (1998), «The Evidence on Class Size », Occasional Paper No. 98-1, W. Allen Wallis Institute of Political Economy, University of Rochester, février. 
HANUSHEK, E.A. et KIM, D. (1995),

«Schooling, Labor Force Quality, and Economic Growth », NBER Working Paper No. 5399, décembre.

HANUSHEK, E.A. et KIMKO, D.D. (2000),

«Schooling, Labor Force Quality, and the Growth of Nations », The American Economic Review, vol. 90, n 5, décembre.

HANUSHEK, E.A. et SOMERS, J. (1999),

«Schooling, Inequality and the Impact of Government », rap port présenté à la conférence «Increasing Income Inequality in America », Texas A\&M University, mars.

HANUSHEK, E.A., KAIN, J. et RIVKIN, S. (1998),

«Teachers, Schools, and Academic Achievement », National Bureau of Economic Research Working Paper 6691, août.

HANUSHEK, E.A., KAIN, J. et RIVKIN, S. (1999),

«Do Higher Salaries Buy Better Teachers? », National Bureau of Economic Research Working Paper No. 7802, mars.

HANUSHEK, E.A., RIVKIN, S. et TAYLOR, L. (1996),

«Aggregation and the Estimated Effects of School Resources », Review of Economics and Statistics, novembre, vol. 78(4), pp. 611-627.

HANUSHEK, E., KAIN, J., MARKMAN, J. et RIVKIN, S. (2000),

«Do Peers Affect Student Achievement? », rapport présenté à la conférence «Empirics of Social Interactions », Brookings Institution, 14-15 janvier.

HAO, L. (1994),

K in Support, Welfare, and O ut-of-Wedlock M others, Garland, New York.

HARBERGER, A. (1998),

«A Vision of the Growth Process », American E conomic Review, vol. 88, $n^{\circ} 1$, mars.

HARMON, F. et WALKER, I. (1995),

«Estimates of the Economic Return to Schooling for the United Kingdom », American E conomic Review, n 85, pp. 12781286.

HARTOG, J. (1997),

«On Returns to Education : Wandering along the Hills of ORU Land », discours pour la LVIIth Conference of the Applied Econometrical Association, Maastricht, mai.

HAVEMAN, R H. et WOLFE, B. (1984),

«Schooling and Economic Well-Being : The Role of Non-market Effects », Journal of H uman Resources, $n^{\circ} 19$, été, pp. 378-407.

HECKMAN, J. (1999),

«Policies to Foster Human Capital », National Bureau of Economic Research Working Paper No. 7288, Cambridge, MA, août.

HELLIWELL, J.F. (1996),

«Economic Growth and Social Capital in Asia », National Bureau of Economic Research Working Paper No. 5470, Cambridge, MA.

HELLIWELL, J.F. (2001),

«The Contribution of Human and Social Capital to Sustained Economic Growth », The Contribution of H uman and Social Capital to Sustained E conomic Growth and Well-being : International Symposium Report, Développement des ressources humaines Canada et OCDE.

HELLIWELL, J.F. et PUTNAM, R. (1999a),

«Economic Growth and Social Capital in Italy », dans P. Dasgupta et I. Serageldin (dir. pub.), Social Capital : A M ultifaceted Perspective, Banque mondiale.

HELLIWELL, J.F. et PUTNAM, R. (1999b),

«Education and Social Capital », NBER Working Paper No. 7121, National Bureau of Economic Research, Cambridge, MA.

HENDERSON, A. et BERLA, N. (1994), A N ew G eneration of Evidence: The F amily is Critical to Student Achievement, National Committee for Citizens in Education, Washington, DC. 
HERS, J. (1998),

« Human Capital and Economic Growth », GPB Report, vol. 98(2).

HESTON, A. et SUMMERS, R. (1988), «A New Set of International Comparisons of Real Product and Price Levels Estimates for 130 Countries, 1950-1985 », Review of Income and Wealth, XXXIV, pp. 1-26.

HESTON, A. et SUMMERS, R. (1991),

«The Penn World Table (Mark 5) : An Expanded Set of International Comparisons, 1950-1988 », Quarterly Journal of E conomics, mai, pp. 327-368.

HIRSCHMAN, A. (1984),

«Against Parsimony : Three Easy Ways of Complicating Economic Analysis », American Economic Review, $n^{\circ} 74$, pp. 88-96.

HJERRPE, R. (1998), «Social Capital and Economic Growth », Discussion paper No. 183, Government Institute for Economic Research (VATT), novembre.

HO SUI-CHU, E. et WILLMS, J.D. (1996),

«The Effects of Parental Involvement on Eighth Grade Achievement », Sociology of Education, n 69, pp. 126-141.

HODGKINSON, V. et WEITZMAN, M. (1988), Giving and Volunteering in the United States: Findings from a National Survey, 1988 E dition, Independent Sector, Washington, D.C.

HOXBY, C. (1994),

«Do Private Schools Provide Competition for Public Schools? », National Bureau of Economic Research Working Paper 4978, New York, décembre.

HUMPHREY, J. et SCHMITZ, H. (1998),

«Trust and Inter-firm Relations in Developing and Transition Economies », The Journal of D evelopment Studies, 34(4), pp. 32-45.

INGLEHART, R. (1997), Modernization and Postmodernization : Cultural, Economic and Political Change in 43 Societies, Princeton University Press, Princeton.

INGLEHART, R. (2000), «Globalization and Postmodern Values », The Washington Q uarterly, hiver.

INOGUCHI, T. (2000), dans Putnam, R. (2000b), Society and Civic Spirit (G esellschaft und Gemeinsinn), Bertelsmann Foundation.

JACOBS, J. (1961),

The Life and D eath of $\mathrm{G}$ reat American Cities, Random House, New York.

JENKINS, H. (1995a),

Education and Production in the United Kingdom, Economics Discussion Paper No. 101, Nuffield College, Oxford.

JENKINS, H. (1995b),

Infrastructure, Education and Productivity : A M ulti-Country Study, DPhil thesis, Nuffield College, Oxford University.

JENSON, J. (1998), «Mapping Social Cohesion: The State of Canadian Research », Canadian Policy Research Networks Study.

JONES, C.I. (1996), «Human Capital, Ideas and Economic Growth », rapport présenté au VIII Villa Mondragone International Economic Seminar on Finance, Research, Education and Growth, Rome, Italie, juin.

JORGENSON, D. et FRAUMENI, B. (1987), «The Accumulation of Human and Non-human Wealth, 1948-1984 », manuscrit non publié, Harvard University.

JOSHI, H., COOKSEY, E., WIGGINS, R., MCCULLOCH, A., VERROPOULOU, G. et CLARKE, L. (1999), "Diverse Family Living Situations and Child Development : A Multi-level Analysis Comparing Longitudinal Evidence from Britain and the United States », International Journal of Law, Policy and the F amily, $n^{\circ} 13$, pp. 292-314. 
KAWACHI, I., KENNEDY, B.P., LOCHNER, K. et PROTHROW-STITH, D. (1997),

« Social Capital, Income Inequality, and Mortality », American Journal of Public Health, vol. 87 (9), pp. 1491-1499.

KEESE, M. et PUYMOYEN, A. (à paraître),

"Changes in Earnings Structure : Some International Comparisons Using the OECD Structure of Earnings Database », Document de la série Politiques du marché du travail et politiques sociales, OCDE, Paris.

KELLAGHAN, T. (1999),

«Educational Disadvantage : An Analysis», rapport présenté au Irish Department of Education and Science Conference of Inspectors, Killarney, 6-8 décembre.

KELLAGHAN, T., SLOANE, K., ALVAREZ, B. et BLOOM, B. (1993),

The Home E nvironment and School Learning. Promoting Parental Involvement in the Education of Children, Jossey-Bass, San Francisco.

KENDRICK, J. (1976), The F ormation and Stocks of Total Capital, Columbia University Press for NBER, New York.

KENKEL, D. (1991),

«Health Behavior, Health Knowledge, and Schooling », Journal of Political Economy, vol. 99(2), pp. 287-305.

KENNEDY, I.B.P., LOCHNER, K. et PROTHROW-STITH, D. (1997),

«Social Capital, Income Inequality, and Mortality », American Journal of Public $\mathrm{H}$ ealth, vol. $87, \mathrm{n}^{\circ} 1$.

KERN, H. (1998),

«Lack of Trust, Surfeit of Trust : Some Causes of the Innovation Crisis in Germany Industry », dans C. Land et R. Bachmann (dir. pub.), Trust within and between O rganizations, Oxford University Press, New York, pp. 203-213.

KING, R. et LEVINE, R. (1994),

«Capital Fundamentalism, Economic Development and Economic Growth », Carnegie-Rochester Series on Public Policy, $n^{\circ} 40$.

KORBIN, J. et COULTON, C. (1997),

«Understanding the Neighborhood Context for Children and Families : Combining Epidemiological and Ethnographic Approaches », dans J. Brooks-Gunn, G.J. Duncan et J.L. Aber (dir. pub.), N eighborhood Poverty, vol. II, Russell Sage Foundation, New York, pp. 65-79.

KNACK, S. (1999), «Social Capital, Growth and Poverty; A Survey of Cross-Country Evidence », Social Capital Initiative, Working Paper No. 7, Banque mondiale.

KNACK, S. (2001),

«Trust, Associational Life and Economic Performance », dans J.F. Helliwell (dir. pub.), The Contribution of Human and Social Capital to Sustained Economic G rowth and Well-being : International Symposium Report, Développement des ressources humaines Canada et OCDE.

KNACK, S. et KEEFER, P. (1997),

«Does Social Capital Have an Economic Payoff? A Cross-Country Investigation », Q uarterly Journal of Economics, vol. 112(4), pp. 1251-1288.

KRUEGER, A. et LINDAHL, M. (1999), «Education for Growth in Swed en and the World », NBER Working Paper No. 7190.

KUZNETS, S. (1962),

dans The N ew Republic, Washington DC, 20 octobre.

KYRIACOU, G. (1991),

Level and G rowth Effects of H uman Capital : A Cross-Country Study of the Convergence H ypothesis, C.V. Starr Centre, Working Paper No. 91-26, New York.

La PORTA, R., LOPEZ-DE-SILANES, F., SHLEIFER, A. et VISHNY, R.W. (1997), «Trust in Large Organisations », American E conomic Review, Papers and Proceedings, vol. 87(2), pp. 333-338.

LANDSBURG, S. (1993),

The Armchair E conomist : E conomics and Everyday Life, The Free Press, New York.

LEANA, C.R. et van BUREN, H.J. (1999),

«Organizational Social Capital and Employment Practices », Academy of Management Review, vol. 24, n 3, pp. 538-555. 
LEE, J.W. et BARRO, R.J. (1997),

«Schooling Quality in a Cross-Section of Countries », NBER Working Paper No. 6198.

LESSER, E.L. (2000),

Knowledge and Social Capital, Butterworth-Heinemann, Boston.

LEVY, F. et MURNANE, R.J. (1999),

«Are there Key Competencies Critical to Economic Success? An Economics Perspective », rapport présenté à un Symposium de I'OCDE sur «La définition et la sélection des compétences », octobre.

LOURY, G. (1987),

«Why Should we Care about Group Inequality? », Social Philosophy and Policy, pp. 249-271.

LUCAS, R.E. (1988),

«On the Mechanisms of Economic Development », Journal of M onetary E conomics, vol. 22.

LUNDVALL, B.-A. et JOHNSON, B. (1994),

«The Learning Economy », Journal of Industry Studies, vol. 1, n² 2, pp. 23-42.

LUNDVALL, B.-A. et MASKELL, P. (1999), «Nation States and Economic Development : From National Systems of Production to National Systems of Knowledge Creation and Learning », dans G.L. Clark et al. (dir. pub.), H andbook of E conomic G eography, chapitre 10, Oxford University Press, Londres.

LYNCH, J., DUE, P., MUNTANER, C. et DAVEY SMITH, G. (2000), "Social Capital - Is it a Good Investment Strategy for Public Health », Journal of E pidemiology and Community H ealth, vol. 54, pp. 404-408.

MADDISON, A. (1987),

«Growth and Slowdown in Advanced Capitalist Economies : Techniques of Quantitative Assessment », Journal of E conomic Literature, $n^{\circ} 25, p p .649-698$.

MADDISON, A. (1991),

D ynamic F orces in Capitalist D evelopment, Oxford University Press, Oxford.

MANKIW, N.G. (1995),

«The Growth of Nations », B rookings Papers on E conomic Activity, No. 1, pp. 275-326.

MANKIW, N.G., ROMER, D. et WEIL, D.N. (1992),

«A Contribution to the Empirics of Economic Growth », Q uarterly Journal of Economics, $n^{\circ}$ 107, pp. 407-437.

MCLANAHAN, S. et SANDEFUR, G.D. (1994),

G rowing U p with a Single Parent: What H urts, What $\mathrm{H}$ elps, Harvard University Press, Cambridge, MA.

MCMAHON, W.W. (2001),

«The Impact of Human Capital on Non-Market Outcomes and Feedbacks on Economic Development in OECD Countries », dans J.F. Helliwell (dir. pub.), The Contribution of H uman and Social Capital to Sustained E conomic G rowth and Well-being : International Symposium Report, Développement des ressources humaines Canada et OCDE.

MINCER, J. (1974),

«Schooling, Experience and Earnings », National Bureau of Economic Research, Cambridge, MA.

MINGAT, A. et TAN, J. (1996),

«The Full Social Returns to Education : Estimates Based on Countries' Economic Growth Performance », Human Capital Development Papers, Banque mondiale, Washington, DC.

MOORE, G. (1990),

«Structural Determinants of Men's and Women's Personal Networks», American Sociological Review, vol. 55, octobre.

MOSTELLER, F. (1995),

"The Tennessee Study of Class Size in the Early School Grades », The Future of Children 5, n², été/automne, pp. 113-127.

MUNTANER, C., LYNCH, J. et SMITH, G.D. (2000),

«Social Capital and the Third Way in Public Health », Critical public Health, vol. 10, $n^{\circ} 2$.

$\overline{\text { C) OCDE } 2001}$ 
MURNANE, R.J. (1975),

Impact of School Resources on the L earning of Inner City Children, Ballinger, Cambrid ge, MA.

MYERS, D. (1999),

«Close Relationships and Quality of Life », dans D. Kahneman, E. Diener et E. Schwartz (dir. pub.), Well-being : The Foundations of $\mathrm{H}$ edonic Psychology, Russell Sage Foundation, New York.

NARAYAN, D. et PRITCHETT, L. (1998),

«Cents and Sociability: Household Income and Social Capital in Rural Tanzania », E conomic D evelopment and Cultural Change, Banque mondiale, Washington.

NEHRU, V. et DHARESHWAR, A. (1994), « New Estimates of Total Factor Productivity Growth for Developing and Industrial Countries », Policy Research Working Paper 1313, Banque mondiale, Washington D.C.

NEHRU, V., SWANSON, E. et DUBEY, A. (1995), «A New Database on Human Capital Stock in Developing and Industrial Countries : Sources, Methodology and Results », Journal of D evelopment E conomics, $n^{\circ}$ 46, pp. 379-401.

NIE, N.H., JUNN, J. et STEHLIK-BARRY, K. (1996),

Education and D emocratic Citizenship in America, University of Chicago Press, Chicago.

NONNEMAN, W. et VANHOUDT, P. (1996),

"A Further Augmentation of the Solow Model and the Empirics of Economic Growth for OECD Countries », Quarterly Journal of E conomics, $n^{\circ} 111$, pp. 943-953.

NORDHAUS, W. et TOBIN, J. (1972), «Is growth obsolete? », In E conomic G rowth, Fiftieth Anniversary Colloquium, vol. 5, NBER, N ew York.

NORTH, D. (1990), Institutions, Institutional Change and E conomic Performance, Cambridge University Press, New York.

NORTON, A. (1998),

«The Welfare State : Depreciating Australia's Social Capital? », Policy, pp. 41-43.

NYE, B. et HEDGES, L. (2000), «The Effects of Small Classes on Academic Achievement : The Results of the Tennessee Class Size Experiment », American Educational Research Journal, vol. 37, n 1, pp. 123-151, printemps.

OCDE (1976),

M esure du bien-être social. Progrès accomplis dans l'élaboration des indicateurs sociaux, Paris.

OCDE (1996),

Réconcilier l'économique et le social. Vers une économie plurielle, Service du développement territorial, Paris.

OCDE (1998),

L'investissement dans le capital humain - Une comparaison internationale, Paris.

OCDE (1999a),

Analyse des politiques d'éducation, Paris.

OCDE (1999b),

Rapport intérimaire relatif au projet triennal de I'OCDE sur le développement durable, Paris.

OCDE (2000a),

Société du savoir et gestion des connaissances, Paris.

OCDE (2000b),

«Liens entre la politique économique et la croissance : constatations au niveau international », projet de rapport pour le Groupe $n^{\circ} 1$, Département des Affaires économiques, Paris.

OCDE (2001),

Analyse des politiques d'éducation, Paris.

OCDE et Département de l'Éducation des États-Unis (1998),

H ow Adults Learn, Actes d'une conférence organisée à Washington, D.C., avril. 
OCDE et Statistique Canada (2000),

La littératie à l'ère de l'information : Rapport final de l'E nquête internationale sur la littératie des adultes, Paris.

OFFE, C. et FUCHS, S. (1998),

«A Decline of Social Capital? - The German Case », dans R. Putnam (2000b), Society and Civic Spirit (G esellschaft und Gemeinsinn), Bertelsmann Foundation.

OHSAKO, T. (1998),

"Learning and Social Participation by Senior Citizens in Japan : Analysis of Major Issues from an International Perspective », dans H ow Adults Learn, Actes d'une conférence organisée à Washington, D.C. et parrainée par I'OCDE et le Département de l'Éducation des États-Unis, avril.

OLSON, M. (1982),

The Rise and Decline of $\mathrm{N}$ ations, Yale University Press, New Haven.

OMORI, T. (2001),

«Balancing Economic Growth with Well-being : Implication of the Japanese Experience », dans J.F. Helliwell (dir. pub.), The Contribution of H uman and Social Capital to Sustained E conomic G rowth and Well-being : International Symposium Report, Développement des ressources humaines Canada et OCDE.

OSBERG, L. (1985),

«The Measurement of Economic Welfare, » dans D. Laidler (coordinateur), Approaches to Economic Well-Being, vol. 26 du Research Program of the Royal Commission of the Economic Union and Development Prospects for Canada (MacDonald Commission), University of Toronto Press, Toronto.

OSBERG, L. (1992),

"Sustainable Social Development », dans R.C. Allen et G. Rosenbluth (dir. pub.), False Promises: The Failure of Conservative E conomics, New Star Books, Vancouver, pp. 227-242.

OSBERG, L. (2001),

«Comparisons of Trends in GDP and Economic Well-being - The Impact of Social Capital », dans J.F. Helliwell (dir. pub.), The Contribution of H uman and Social Capital to Sustained E conomic G rowth and Well-being : International Symposium Report, Développement des ressources humaines Canada et OCDE.

PAINTER, G. et LEVINE, D. (1999),

"Family Structure and Youths' Outcomes: Which Correlations are Causal? », Institute of Industrial Relations, Working Paper Series, $n^{\circ} 69$, septembre.

PEARCE, D. et ATKINSON, G. (1997),

«The Concept of Sustainable Development: An Evaluation of its Usefulness Ten Years after Bruntland », Centre for Social and Economic Research on the Global Environment, University College London and University of East Anglia.

PICCIOTTO, R. (1998),

"Gender and Social Capital », Présentation donnée lors du Gender and Development Workshop, Banque mondiale, avril (http://www.worldbank.org/html/prmge/know/gendev/).

PORTER, M. (1990), The Competitive Advantage of N ations, The Free Press, New York.

PORTES, A. (1998),

«Social Capital : Its Origins and Applications in Modern Sociology », Annual Review of Sociology, n² 24, pp. 1-24.

PORTES, A. et LANDOLT, P. (1996),

«The Downside to Social Capital », The American Prospect, n²6, pp. 18-21, 94, mai-juin.

PORTES, A. et SENSENBRENNER, J. (1993),

«Embeddedness and Immigration : Notes on the Social Determinants of Economic Action », American Journal of Sociology, 98(6), pp. 1320-1350.

POWELL, D.R. (1989),

«Families and Early Child hood Progams », National Association for the Education of Young Children, Washington D.C.

POWELL, W. et SMITH-DOERR, L. (1994),

"Networks and Economic Life », dans N.J. Smelser et R. Swedberg (dir. pub.), The Handbook of E conomic Sociology, Princeton University Press, Princeton, pp. 368-402. 
PRITCHETT, L. (1999), Where H as All the E ducation G one?, Banque mond iale, Washington D.C.

PROGRAMME DES NATIONS UNIES POUR LE DÉVELOPPEMENT (1990), Rapport mondial sur le développement humain, 1990, Oxford University Press, New York, NY.

PROGRAMME DES NATIONS UNIES POUR LE DÉVELOPPEMENT (2000), Rapport mondial sur le développement humain, 2000, Oxford University Press, New York, NY.

PSACHAROPOULOS, G. (1994), «Returns to Investment in Education : A Global Update », World D evelopment, vol. 22(9), pp. 1325-1343.

PUTNAM, R. (1993), M aking Democracy Work, Princeton University Press, Princeton.

PUTNAM, R.D. (1995), «Bowling Alone : America's Declining Social Capital », Journal of Democracy, vol. 6, n 1, pp. 65-78 (http ://muse.jhu.edu/demo/journal_of_democracy/v006/putnam.html).

PUTNAM, R. (2000a), Bowling Alone : The Collapse and Revival of American Community, Simon Schuster, New York.

PUTNAM, R. (2000b), Society and Civic Spirit (Gesellschaft und Gemeinsinn), Bertelsmann Foundation.

PUTNAM, R. (2001), «Social Capital : Measurement and Consequences», dans J.F. Helliwell (dir. pub.), The Contribution of Human and Social Capital to Sustained Economic G rowth and Well-being : International Symposium Report, Développement des ressources humaines Canada et OCDE.

RAUDENBUSH, S.W. et KASIM, R.M. (1998), «Cognitive Skill and Economic Inequality : Findings from the National Adult Literacy Survey », Harvard E ducational Review, vol. 68 (1), pp. 33-79.

RITZEN, J. (2001), «Social Cohesion, Public Policy, and Economic Growth : Implications for OECD Countries », dans J.F. Helliwell (dir. pub.), The Contribution of H uman and Social Capital to Sustained E conomic G rowth and Well-being : International Symposium Report, Développement des ressources humaines Canada et OCDE.

RIVERA-BATIZ, F.L. (1994), «Quantitative Literacy and the Likelihood of Employment among Young Adults in the United States », Journal of Human Resources, vol. XXVII, n² 2, pp. 313-328.

RIVKIN, S.G., HANUSHEK, E.A. et KAIN, J.F. (1998), «Teachers, Schools, and Academic Achievement », Rapport présenté à la réunion annuelle de la Econometric Society, Chicago, janvier.

RIZZO, J. et ZECKHAUSER, R. (1992),

«Advertising and the Price, Quantity, and Quality of Primary Care Physician Services », Journal of H uman Resources, 27(3), pp. 381-421.

RODRIK, D. (1998), «Where Did All the Growth Go? External Shocks, Social Conflict and Growth Collapses », NBER Working Paper No. 6350 .

RODRIK, D. (2000), «Development Strategies for the Next Century », Rapport présenté à la conférence « Developing Economies in the 21st Century », Institute for Developing Economies, Japan External Trade organization, janvier (http://www.ksg.harvard.edu/rod rik/d evstrat.PDF).

ROMER, P.M. (1990a),

"Human Capital and Growth : Theory and Evidence », Carnegie-Rochester Conference Series on Public Policy, $n^{\circ} 32$, pp. 251-286.

ROMER, P.M. (1990b), «Endogenous Technological Change », Journal of Political E conomy, 98(5), Partie 2, pp. 71-102. 
ROSE, R. (2000),

«How Much Does Social Capital Add to Individual Health? A Survey Study of Russians », Social Science and M edicine, I-15 Pergamon.

ROTHSTEIN, B. (1998),

«Social Capital in the Social Democratic State - The Swedish Model and Civil Society », Department of Political Science, Göteborg University, Suède.

ROUSE, C.E. (1998),

«Schools and Student Achievement : More Evidence from the Milwaukee Parental Choice Program », Economic Policy Review, mars, 4(1), pp. 61-78.

RUNYAN, D., HUNTER, W. et al. (1998), «Children Who Prosper in Unfavorable Environments : The Relationship to Social Capital », Pediatrics, 101, pp. 12-18.

SALA-I-MARTIN, X. (1997), «I Just Ran Four Million Regressions », NBER Working Paper No. 6252.

SAMPSON, R. (1995), «The Community », dans J. Wilson et J. Petersilia (dir. pub.), Crime, Institute for Contemporary Studies Press, San Francisco, pp. 193-216.

SAMPSON, R. et MORENOFF, J. (1997), «Ecological Perspectives on the Neighborhood Context of Urban Poverty : Past and Present », dans J. Brooks-Gunn, G. Duncan et J.L. Aber (dir. pub.), N eighborhood Poverty : Volume II, Russell Sage Foundation, New York, pp. 1-22.

SAMPSON, R., RAUDENBUSH, S. et EARLS, F. (1997),

«Crime : A Multilevel Study of Collective Efficacy », Science, 277, 15 août.

SANDEFUR, G.D., MCLANAHAN, S. et WOJTKIEWICZ, R.A. (1989), «Race and Ethnicity, Family Structure, and High School Graduation », Discussion Paper No. 893-889, Institute for Research on Poverty.

SCARPETTA, S., BASSANINI, A., PILAT, D. et SCHREYER, P. (2000), «Economic Growth in the OECD Area : Recent Trends at the Aggregate and Sectoral Level », Working Paper No. 248, Département des Affaires économiques de I'OCDE.

SCHULLER, T., BYNNER, J., GREEN, A., BLACKWELL, L., HAMMOND, C. et PRESTON, J. (2001), «Modelling and Measuring the Wider Benefits of Learning : An Initial Synthesis », Centre for Research on the Wider Benefits of Learning Institute of Education/Birkbeck College.

SEN, A. (1987),

The Standard of Living, Cambridge University Press, Cambridge.

SHIMADA, H. (1988), «Hyu-man Uea no Keizaigaku (Economics of Humanware) », dans Omori (2001), Iwanami.

SHLEIFER, A. (1998), «State Versus Private Ownership », Journal of E conomics Perspectives, 12 (4), pp. 133-150.

SIMONS, R. (1996), Understanding D ifferences between Divorced and Intact F amilies: Stress, Interaction and Child O utcome, Thousand Oaks, CA, Sage.

SMITH, A. (1776), La richesse des nations, Livre I.

SOLOW, R.M. (1956),

«A Contribution to the Theory of Economic Growth », Q uarterly Journal of Economics, 70, pp. 65-94.

SPENCE, A. (1973),

«Job Market Signalling», Q uarterly Journal of E conomics 87, pp. 355-374.

STEEDMAN, H. (1996), «Measuring the Quality of Educational Outputs : A N ote », Center for Economic Performance, Discussion paper No. 302, LSE.

TEACHMAN, J., PAASCH, K. et CARVER, K. (1999),

«Social Capital and the Generation of Human Capital », Social Forces, 75. 
TEMPLE, J. (1999a),

«The New Growth Evidence », Journal of E conomic Literature, mars, 37(1), pp. 112-156.

TEMPLE, J. (1999b), «A Positive Effect of Human Capital on Growth », E conomics Letters, 66, pp. 131-134.

TEMPLE, J. (2001),

«Growth Effects of Education and Social Capital in the OECD », dans J.F. Helliwell (dir. pub.), The Contribution of H uman and Social Capital to Sustained E conomic G rowth and Well-being : International Symposium Report, Développement des ressources humaines Canada et OCDE.

TEMPLE, J. et JOHNSON, P. (1998),

«Social Capability and Economic Growth », Q uarterly Journal of E conomics, août, pp. 965-988.

UZZI, B. (1996),

«The Sources and Consequences of Embeddedness for the Economic Performance of Organizations : The Network Effect », American Sociological Review, 61(4), pp. 674-698.

UZZI, B. (1997), «Social Structure and Competition in Inter-firm Networks : The Paradox of Embeddedness », Administrative Science Quarterly, 42(1), pp. 35-67.

Van ARK, B. et de JONG, H. (1996),

"Accounting for Economic Growth in the Netherlands since 1913 », University of Groningen Research Memorandum, GD-26.

VERBA, S., SCHLOZMAN, K.L. et BRADY, H.E. (1995), Voice and Equality : Civic Voluntarism in American Politics, Harvard University Press, Cambridge, M.A.

WEISS, A. (1995),

« Human capital vs. signalling explanations of wages », Journal of E conomic Perspectives, 9(4), pp. 133-154.

WHITE, M. et KAUFMAN, G. (1997), «Language Usage, Social Capital, and School Completion among Immigrants and Native-born Ethnic Groups », Social Science Q uarterly, 78(2), pp. 385-393.

WILKINSON, R. (1996), Unhealthy Societies: The Afflictions of Inequality, Routledge, Londres.

WILLMS, J.D. (1999), «Proposal for the Measurement of Socioeconomic Status for the Focused Component of the Student Questionnaire for the OECD Programme for International Student Assessment (OECD-PISA) », The Canadian Research Institute for Social Policy (CRISP), University of New Brunswick.

WILLMS, J. D. (2001), «Three Hypotheses about Community Effects », dans J.F. Helliwell (dir. pub.), The Contribution of H uman and Social Capital to Sustained E conomic Growth and Well-being : International Symposium Report, Développement des ressources humaines Canada et OCDE.

WOLFE, B. et HAVEMAN, R. (2001),

«Accounting for the Social and Non-market Benefits of Education », dans J.F. Helliwell (dir. pub.), The Contribution of $\mathrm{H}$ uman and Social Capital to Sustained E conomic $\mathrm{G}$ rowth and Well-being : International Symposium Report, Développement des ressources humaines Canada et OCDE.

WOLFE, B.L. et ZUVEKAS, S. (1997), $\mathrm{N}$ onmarket $\mathrm{O}$ utcomes of Schooling, University of Wisconsin, Madison, polycopié.

WOLFF, E.N. et M. GITTLEMAN (1993), «The Role of Education in Productivity Convergence : Does Higher Education Matter? », dans A. Szimai, B. Van Ark et D. Pilat (dir. pub.), Explaining E conomic G rowth, Elsevier Science Publishers.

WOOLCOCK, M. (1998),

«Social Capital and Economic Development : Toward a Theoretical Synthesis and Policy Framework », Theory and Society, 27(2), pp. 151-208. 
WOOLCOCK, M. (1999),

«Social Capital : The State of the Notion », Rapport présenté à un séminaire multidisciplinaire sur Social Capital : Global and Local Perspectives, Helsinki, 15 avril.

WOOLCOCK, M. (2001),

«The Place of Social Capital in Understanding Social and Economic Outcomes », dans J.F. Helliwell (dir. pub.), The Contribution of H uman and Social Capital to Sustained E conomic G rowth and W ell-being : International Symposium Report, Développement des ressources humaines Canada et OCDE.

WORMS, J.P. (1998a),

«Old and New Civic and Social Ties in France », dans Putnam (2000b).

WORMS, J.P. (1998b),

«La crise du lien social, le problème du chaînon manquant », Empan, n 32, décembre 1998, pp. 94-104.

WORMS, J.P. (2000),

«Old and New Civic and Social Ties in France », dans R. Putnam (dir. pub.), Society and Civic Spirit (Gesellschaft und Gemeinsinn), Bertelsmann Found ation.

WOSSMANN, L. (2000),

«Schooling Resources, Educational Institutions and Student Performance : The International Evidence », Kiel Working Paper $n^{\circ}$ 983, mai.

YANKELOVICH, D. (1981),

N ew Rules : Searching for Self-fulfillment in a World Turned U pside D own, Bantam Books, New York. 
LES ÉDITIONS DE L'OCDE, 2, rue André-Pascal, 75775 PARIS CEDEX 16 IMPRIMÉ EN FRANCE

(96 2001012 P) ISBN 92-64-28589-X - N 516462001 